\title{
Population structure of Magnaporthe oryzae from different geographic regions and interaction transcriptomes with rice genotypes at high temperature
}

\author{
Dissertation \\ to obtain the Ph.D. degree \\ in the International Ph.D. Program for Agricultural Sciences in Goettingen (IPAG) \\ at the Faculty of Agricultural Sciences, \\ Georg-August-University Göttingen, Germany
}

Geoffrey Onaga

Born in Ngora, Uganda

Göttingen, May 2014 
D7

1. Name of referee: Prof. Dr. Andreas von Tiedemann

2. Name of co-referee: Prof. Dr. Kerstin Wydra

Date of disputation: $9^{\text {th }}$ of July 2014 


\section{Contents}

Contents. III

Chapter 1: Introduction........................................................................................................................... 6

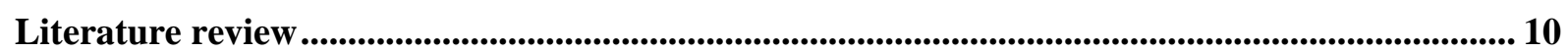

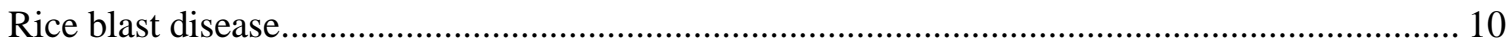

Pathogenicity factors and effector molecules of $M$. oryzae …...................................................... 11

Variability and population structure studies on $M$. oryzae ……..................................................... 14

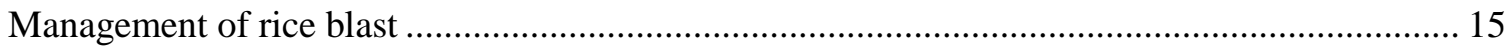

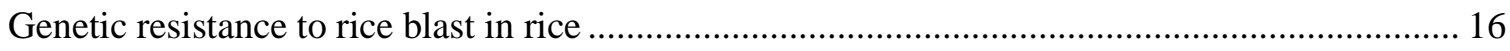

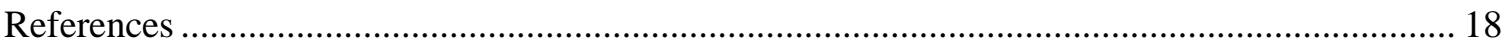

Chapter 2: Rice NILs carrying Pi54 in LTH and Co39 backgrounds confer resistance to Magnaporthe oryzae at $35^{\circ} \mathrm{C}$ but show contrasting reactions at $28^{\circ} \mathrm{C}^{*}$......................................... 26

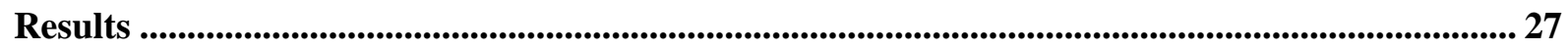

Phenotypic performance of infected plants after high temperature exposure …........................... 27

Transcription profiles of $\mathrm{CO}$ and LT infected with M. oryzae at $28^{\circ} \mathrm{C}$ and $35^{\circ} \mathrm{C}$......................... 27

Gene ontology enrichment analysis of DEGs from infected samples at both temperatures.......... 28

Gene expression profiles in LT and CO in response to only high temperature $\left(35^{\circ} \mathrm{C}\right) \ldots \ldots \ldots \ldots \ldots . . . . .29$

Expression of peptidyl-prolyl isomerase is elevated in response to $35^{\circ} \mathrm{C}$ plus $M$. oryzae infection

Differentially expressed resistance (R) gene and R gene analogues (RGAs).............................. 31

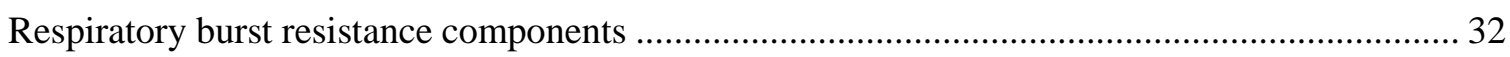

Hormone signaling pathway in relation to high temperature induced resistance .......................... 33

Activated signaling pathways involved in rice defense against pathogen attack ......................... 35

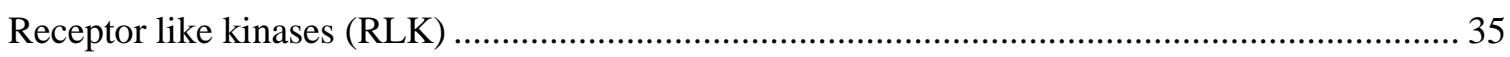

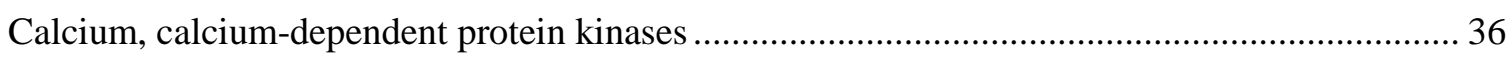

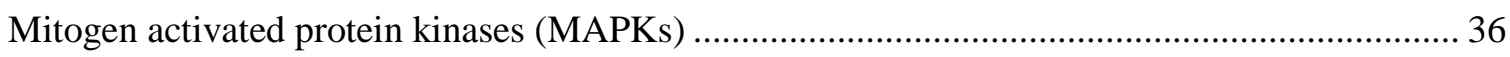

Transcription factors (TFs) induced during high temperature stress ......................................... 37

Homeobox (HB), bZIP, AP2-EREBP and MYB transcription factor encoding genes are highly

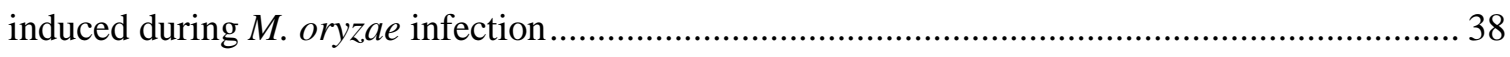

Aspartic proteinases and antimicrobial compounds are highly up-regulated during M.oryzae

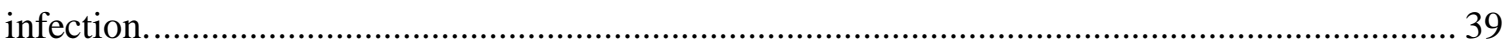

Transcriptional regulation of the TCA cycle, carbon and amino acid metabolism ........................ 40

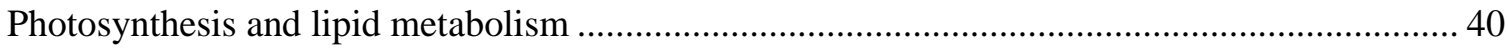

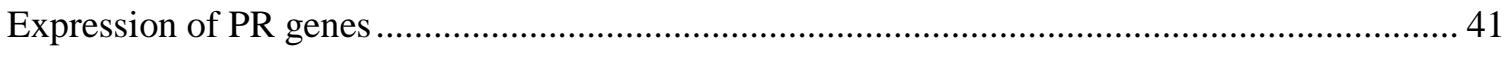

Expression of genes involved in secondary metabolism during pathogen infection ...................... 42

Validation of differential Gene Expression of known temperature and infection responsive rice

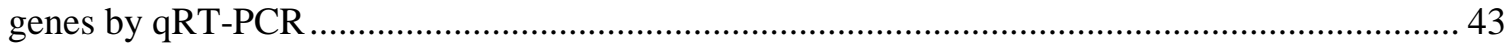

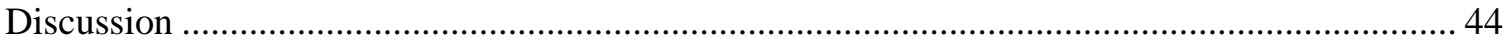




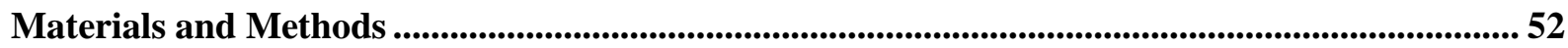

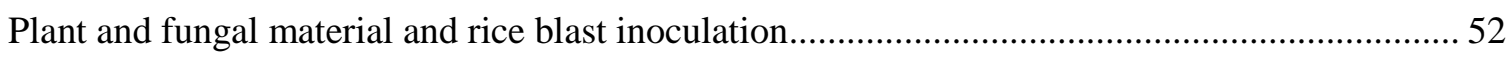

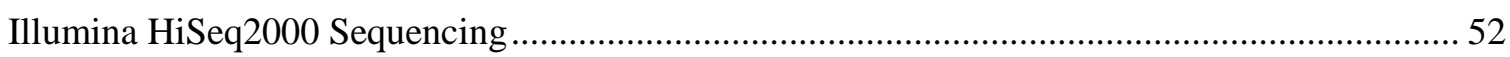

Preprocessing, Mapping, and analysis of illumina reads and subsequent gene expression data ... 53

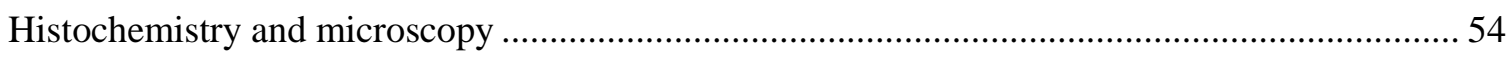

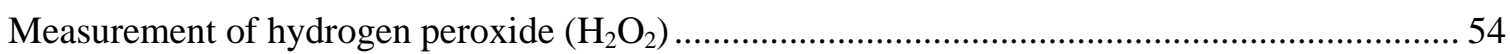

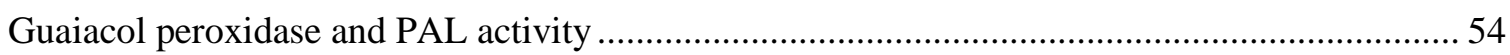

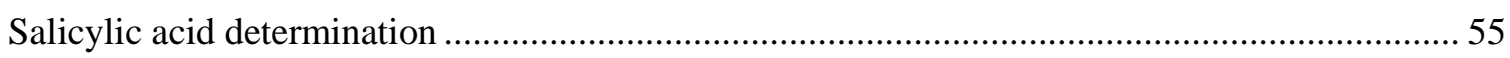

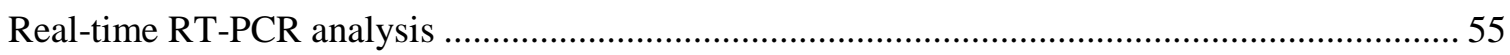

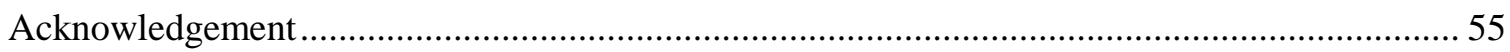

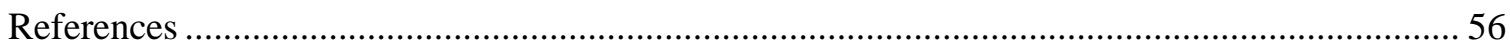

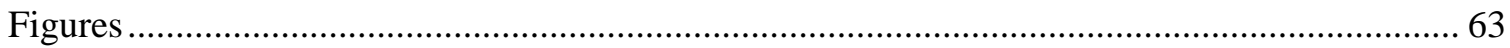

Chapter 3: Transcript profiles of Magnaporthe oryzae in planta expressed genes and rice defense response genes in plants pre-exposed to high temperature ...................................................................... 78

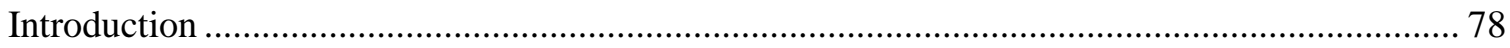

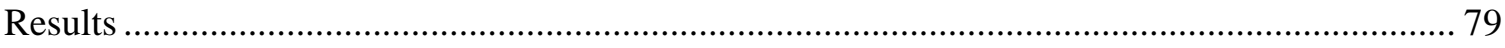

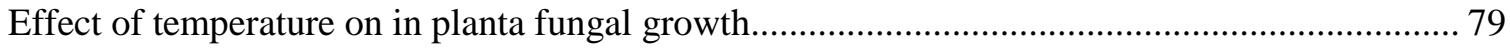

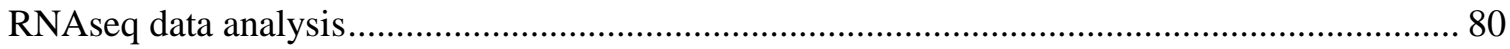

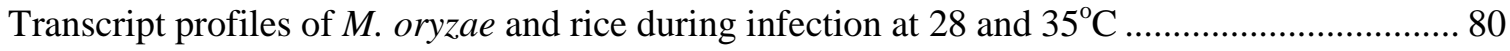

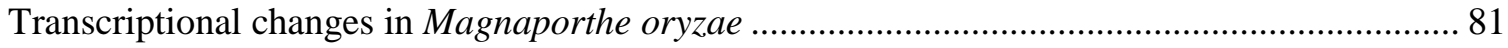

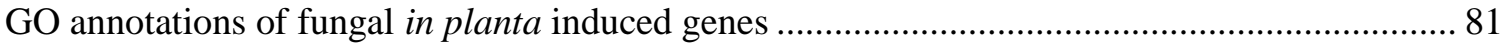

High temperature influence on Known pathogenicity genes....................................................... 82

Analysis of $M$. oryzae secretome during infection at high temperature …...................................... 82

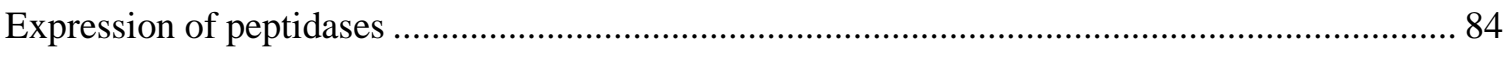

Increased expression levels of previously identified and novel secreted proteins of M. oryzae at

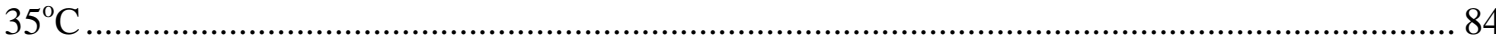

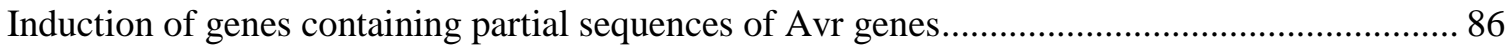

Effect of high temperature stress on lipid metabolism of $M$. oryzae during infection ................... 86

Effect of high temperature stress on carbohydrate metabolism of $M$. oryzae during infection..... 87

Stress and secondary metabolism induced genes during infection at high temperature................. 89

Magnaporthe oryzae induced genes encoding for transport proteins........................................... 90

Regulation of transcription factors in $M$. oryzae during infection at high temperature.................. 91

Temperature-responsive, promoter motifs identified for induced genes specific to Nip35i ......... 92

Rice plant reactions in response to HT and M. oryzae infection............................................... 92

GO enrichment and transcript abundance in rice during M. oryzae infection.............................. 92

Activated signaling pathways involved in rice defense against pathogen attack at high temperature 
Hormone signaling pathway in relation to high temperature in Nipponbare ................................ 94

Induced transcription factors in rice during M. oryzae infection at 35 and $28^{\circ} \mathrm{C}$........................ 95

Genes involved in secondary metabolism in rice response to pathogen attack .............................. 96

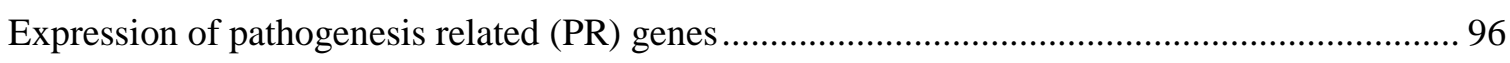

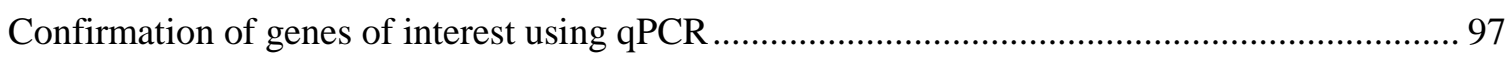

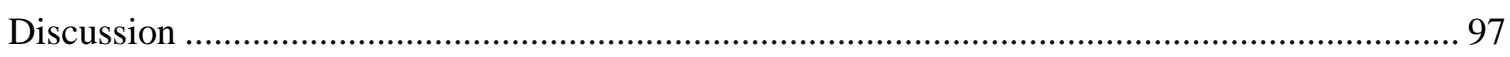

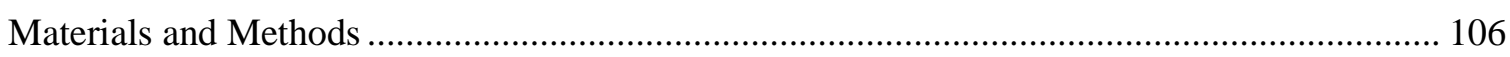

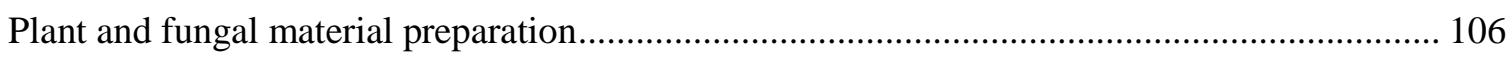

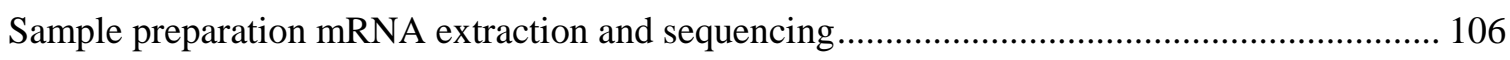

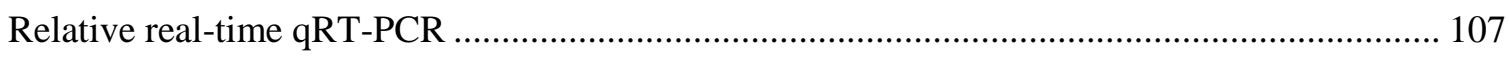

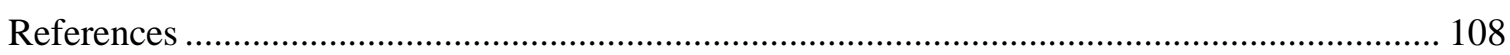

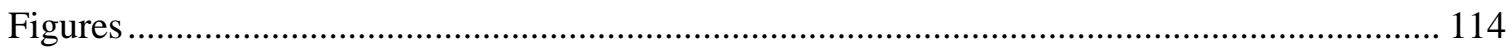

Chapter 4: Population structure, pathogenicity and mating type distribution of Magnaporthe oryzae isolates from East Africa ........................................................................................................................ 124

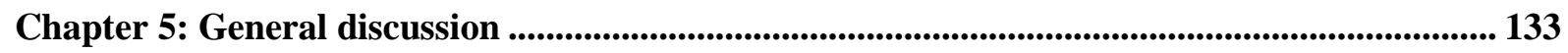

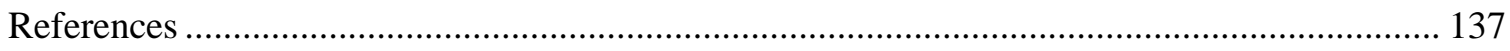

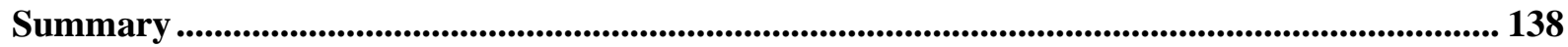

Appendix 1 ................................................................................................................................................. 141

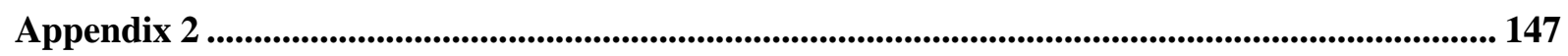

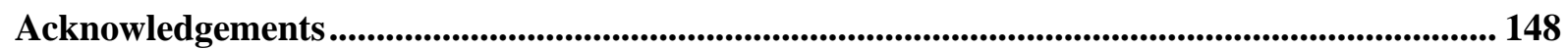

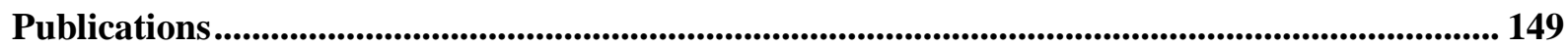

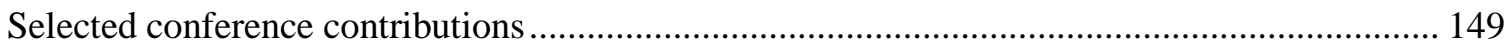

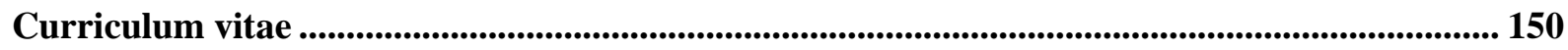

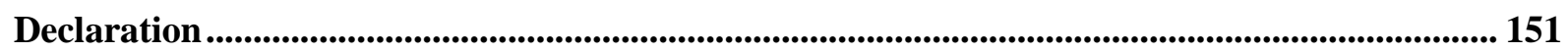


Chapter 1: Introduction

It is currently assumed that climate change could lead to global warming. Factors associated with climate change are considered to include an increase in average temperatures and alteration of wind, rainfall regimes, humidity and carbondioxide concentrations (Bokszczanin and Fragkostefanakis, 2013). The effects of altering these climatic factors on the pathogen cycles, epidemiology and plant host resistance remains less clear. Although several authors have attempted to summarize the evidence in systematic reviews, overlapping evaluations are often presented, with several reports indicating that climate change could probably cause variation even in individual pathosystems, with positive, negative and neutral interactions predicted to occur (Pangga et al., 2011; Juroszek and von Tiedemann, 2012). With respect to temperature, there is evidence that elevated temperature effects on pathosystems may vary not only at the host genotypic level but also at the resistance $(\mathrm{R})$ gene -pathogen interaction level (Gousseau et al., 1985; Lain, 2007; Webb et al., 2010; Jorgensen, 2012). Such variation indicates that plants have some parallels in their defense response to pathogens.

Rice is a major host of Magnaporthe oryzae, the most destructive pathogen of rice worldwide (Xue et al., 2012). The rice-M. oryzae pathosystem is well-studied at both the phenotypic and genomic level. The scientific advancement in both organisms makes them suitable for understanding the mechanisms underlying plant pathogen interaction at high temperature. The genomes of both organisms have also been sequenced, which enables genomic studies of their interaction (IRGSP, 2005; Dean et al., 2005). The sequenced genome of rice is represented by two rice varieties, Nipponbare from Japan and 93-11 from China, belonging to two major Asian cultivated rice subspecies, Oryza sativa L. japonica and indica, respectively. The sequenced genome of $M$. oryzae is represented by a rice-infecting, fully fertile strain 70-15 (Mat1-1), which was obtained by numerous back crosses to the wild isolate Guy 11 (Leung et al., 1988; Dean et al., 2005).

The primary breeding tool for rice blast management is to introduce resistance $(\mathrm{R})$ genes into elite rice cultivars. The hallmark of $\mathrm{R}$ gene resistance is hypersensitive response, characterized by necrotic lesions resulting from localized host cell death at the site of infection. The hypersensitive response is connected to the induction of hydrogen peroxide, salicylic acid and jasmonic acid. These molecules are well known signaling compounds which play a role in the induction of PR genes, cell wall phenolics, phytoalexins and callose deposition. However, R gene mediated resistance has been shown to break down within 2-3 
years after varietal release. This has been associated with mutations or functional loss of the corresponding Avr genes, and selection of novel virulence factors in a pathogen that could evade detection (Wu et al., 2015).

Effectiveness of $\mathrm{R}$ genes has been shown to vary with the genetic backgrounds (Palloix et al., 2009). For instance, in rice the background of japonica ecotypes have been shown to be more resistant to Xoo strains as compared to the indica background (Cao et al., 2007). Similar findings have been reported in Arabidopsis, in which the function of an allele of the Arabidopsis $R$ gene RPS2 was found to be influenced by the genetic background (Banerjee et al., 2001). This suggests that substantial differences in the genomic regions controlling disease resistance between the two ecotypes exist, and could be interesting experimental situation to study.

Notwithstanding these differences in resistance patterns, little is known about how the two rice subspecies interact with $M$. oryzae at elevated temperature. Although a risk analysis of rice blast epidemics and plant growth associated with climate change previously suggested increased blast severity in the cool sub-tropical zones of Asia (Luo et al., 1998), this study did not consider the differences associated with $O$. japonica and $O$. indica rice subspecies in response to pathogens. Thus, to date, we know little about the infection of rice by $M$. oryzae at elevated temperatures, as influenced by $O$. japonica and $O$. indica genetics. Besides this, it is not known whether the phenotypic differences associated with the host response to M. oryzae infection at high temperature are due to $\mathrm{R}$ genes or the genetic background in which the $\mathrm{R}$ gene is introgressed. Because $M$. oryzae infects rice by regulating protein secretion, which enables the pathogen to either avoid recognition by the plant resistance proteins or to turn off plant defense (Giraldo et al., 2013), it is expected that elevated temperature may also affect $M$. oryzae pathogenicity. However, the direction of high temperature effect on pathogenicity is not well understood.

The recent developments in high-throughput RNA-Seq, including Solexa/Illumina and $\mathrm{ABI} / \mathrm{SOLiD}$, has enhanced the value of transcriptomics and offers effective means for gene expression profiling and discovery of novel transcripts (Garber et al., 2011). These technologies provide a new opportunity for understanding the molecular basis of virulence in pathogens as well as understanding key host defense responses at the same time. Recently, RNA-Seq was used to understand the in planta transcriptome changes in M. oryzae at $24 \mathrm{~h}$ 
after infection and during appressorium development (Kawahara et al., 2012; Soanes et al., 2012). These studies provided insights into molecular processes regulated by M. oryzae during infection, and were helpful in understanding the early events associated with M. oryzae infection. The same authors analyzed the reaction of rice host plant to $M$. oryzae at the transcriptome level and found several plant genes and pathways affected by $M$. oryzae infection. Notwithstanding the identified transcriptional changes associated with rice host plant-M. Oryzae interaction reported before, the use of RNA-seq to understand the changes in gene expression, as influence by the genetic backgound, high temperature and M. oryzae infection remains unclear.

On the other hand, understanding host-pathogen interaction is greatly dependent on the knowledge of the diversity of the pathogen at the field level (Banniza et al., 1999). Hundreds of pathotypes have been reported in various $M$. oryzae rice infecting populations, which cause widespread epidemics (Urashima, 2001). It is expected that the recent warming trend of about $0.5^{\circ} \mathrm{C}$ in East Africa (Hepworth and Goulden, 2008) may have caused emergence of virulent M. oryzae strains, leading to episodes of blast epidemics, including the blast outbreak observed in the farmers' fields in Kenya in 2009 (Kihoro et al., 2009). Because no prior genetic diversity studies have been conducted on M. oryzae in East Africa, deciphering the current status of $M$. oryzae population structure will provide a basis for future studies on climatic factors influencing this pathogen's biology.

Our goal for this research therefore was to better understand the effect of high temperature on phenotypic and transcriptional changes in the rice- $M$. oryzae interaction, and explore the pathogen population structure in less studied areas of East Africa compared to populations of typical rice blast regions in West Africa and the Philippines.

The first objective of this work was to determine transcriptome profiles and biochemical changes that relate to temperature induced phenotypic defense alterations in the rice- $M$. oryzae interaction using $O$. japonica and $O$. indica genetic backgrounds commonly used for the development of rice NILs. We used RNA-seq to test whether increase in temperature from $28^{\circ} \mathrm{C}$ to $35^{\circ} \mathrm{C}$ has an influence on the interaction of M. oryzae with Li-Jiang-Xin-Tuan-He-Gu (LT; Oryza japonica) and Co39 (CO; Oryza indica), carrying the resistance gene Pi54, at 48 hours post inoculation (hpi). Pi54 encodes a nucleotide binding site-leucine rich repeat (NBSLRR) domain with a small zinc finger domain, and was previously reported to be expressed 
constitutively at a basal level in both resistant transgenic as well as susceptible native rice lines up to 48 hpi (Gupta et al., 2012). The phenotypic reactions and transcriptome profiles allowed us to identify the resistant background and the genes and biological processes which were altered during the interaction between M. oryzae, Pi54, genetic backgrounds and high temperature. We also measured the levels of salicylic acid (SA), hydrogen peroxide $\left(\mathrm{H}_{2} \mathrm{O}_{2}\right)$, peroxidase (POX) and phenylalanine ammonia lyase (PAL) in attempts to determine whether gene expression data correlate with defense related biochemical changes. The results of these studies are included in chapter 2.

The second objective of this work was to simultaneously determine the transcript profiles of M. oryzae in planta expressed genes and rice defense response at high temperature using the japonica $\mathrm{cv}$. Nipponbare and $M$. oryzae isolate TAN211.16. TAN211.16 was selected for transcriptome sequencing because it evoked consistent disease reaction scores on most of the monogenic lines tested in the greenhouse. We examined the transcript profiles of TAN211.16 and Nipponbare at $28^{\circ} \mathrm{C}$ and $35^{\circ} \mathrm{C}$ to identify the significant biological processes and genes that relate to pathogen infection, survival and adaptation to the host environment at $48 \mathrm{hpi}$. We searched for genes encoding for known pathogenicity factors, the secretome and/or effectors, transcription factors, primary metabolism and secondary metabolism and compared their induction during infection at $35^{\circ} \mathrm{C}$ to infection at $28^{\circ} \mathrm{C}$. On the other hand, activated signaling pathways associated with defense response in rice were examined to understand the gene expression differences that occur during infection at $28^{\circ} \mathrm{C}$ compared to $35^{\circ} \mathrm{C}$. The results of these studies are included in Chapter 3.

M. oryzae is relatively less studied in East Africa, but considered a threat to rice production. In the third objective, we were interested in understanding the population structure and active evolutionary forces acting on M. oryzae populations in East Africa. We used isolates collected in 2009 and 2011 from three East African countries: Rwanda, Uganda and Tanzania. For comparative purposes, isolates from West Africa and the Philippines were included. A total of 88 isolates were subjected to AFLP analysis. The data were used to estimate gene diversity, genetic distances, genetic differentiation and to make indirect measures of gene flow between local and/or regional population groups. The pathogenicity and mating type distribution for the isolates analyzed were also determined. The results of this study are included in chapter 4 .

The last part of this dissertation constitutes the general discussion. The summary of the main findings and potential contributions of this research work to the scientific community are also presented at the end. 
Literature review

\section{Rice blast disease}

Rice blast, caused by $M$. oryzae, is a filamentous heterothallic ascomycete in the order Diaporthales and family Magnaporthaceae (Cannon, 1994). The naming "Magnaporthe grisea" was previously used to encompass all isolates infecting rice and other grasses (Bar, 1977). However, the name M. grisea was recently changed to M. oryzae based on gene tree topology derived from sections of actin, beta-tubulin, and calmodulin gene sequences, and the inability of isolates infecting rice to interbreed with Digitaria isolates (Couch and Kohn, 2002). This was consistent with previous reports that $M$. oryzae is adapted to rice, and probably did not co-evolve with other graminaceous species (Kato et al., 2000). Moreover, high pathogenicity levels on the tracked host and reduced virulence on closely related hosts was indicative of host-specialization (Couch et al., 2005).

Magnaporthe oryzae is present in almost every rice growing area in the world. The disease has been reported to cause yield losses from the hot and dry climate of California and Iraq (Ou, 1985; Greer et al., 1997) to the cool temperate continental climate of Italy. Yield losses of $10 \%-30 \%$ are typical, although regional epidemics can lead to losses ranging from $70 \%-$ $80 \%$ when predisposing factors favor epidemic development (Piotti et al., 2005). Magnaporthe oryzae is polycyclic, with several generations in a single rice crop cycle. Crop debris and infected seeds are the most common sources of primary inoculum, while secondary spread occurs through splash or wind dispersed conidia following foliar infections. The disease is favored by frequent occurrence of cloudy days with humidity ranging from $80 \%-$ $100 \%$. Rice is most susceptible when young plants hold 3 to 4 leaves and during flowering. Infection of rice plants starts with the landing of airborne conidia on the rice leaf cuticle, followed by germination by producing a germ tube which elaborates into an appressorium (Howard and Valent, 1996). When appressorium is fully developed, turgor generated force ruptures the leaf cuticle and an infection peg emerges from its base, which penetrates the leaf surface. The infection peg differentiates into a series of bulbous, branched infectious hyphae soon after plant infection. Four to five days after infection, first necrotic lesions appear simultaneously with aerial conidiophores. The lesions progress and coalesce to form larger lesions which sporulate after six to ten days (Wang et al., 2005). Sporulation is highly favored by the presence of moisture on infected leaf surfaces and by temperatures ranging from $25^{\circ} \mathrm{C}-$ $30^{\circ} \mathrm{C}$. Cloudy days and extended periods of high humidity also favor sporulation. All foliar tissues and panicles are subject to infection. The leaf lesions are diamond-shaped, dusty 
grayish with a pale chlorotic margin and a necrotic centre and can be longer than $1 \mathrm{~cm}$ (Figure 1.1; Wilson and Talbot, 2009), whereas panicle/stem lesions are mainly necrotic. A recent report also shows that $M$. oryzae attacks the roots (Sesma and Osbourn, 2004). However, the root symptom development studies have received less attention.

\section{Pathogenicity factors and effector molecules of $M$. oryzae}

Pathogenicity is the ability of an organism to cause disease or harm the host. This ability represents genetic components of the pathogen that promote a compatible host-pathogen interaction. Magnaporthe oryzae is classified as a hemibiotroph whereby an initial biotrophic phase, characterized by penetrating the host cell without killing, is followed by a necrotrophic phase, characterized by secreting enzymes and probably toxins that kill and degrade host tissues. This infection strategy is accomplished by employing several factors to colonize and infect rice, for review see (Fernandez and Wilson, 2014a). Successful infection is initiated by a thigmotropic response to a hard surface, which is regulated by regulator of $\mathrm{G}$ protein signaling (Rgsl; Liu et al., 2007). A hard and hydrophobic surface also activates G proteincoupled receptor protein Pth11 (DeZwaan et al., 1999). Both Rgsl and Pth11, in the presence of cyclase-associated protein ( Capl), activate adenylate cyclase Macl via G protein signaling. Activated Macl generates cyclic adenosine monophosphate (cAMP), a process that activates cAMP-dependent protein kinase A ( $c P K A)$ required for initiation of appressorium formation.

Recruitment of mitogen activated protein kinase (Pmkl), which acts downstream of Mst11, Mst7 and an adaptor protein Mst50 (Wilson and Talbot 2009; Li et al., 2012), is required for full appressorium formation. Besides this, two proteins, MoMsb2 and MoShol, are reported to act upstream of Mst11 as hydrophobicity and cutin monomer sensors (Liu et al., 2011). Moreover, two intermediate proteins orthologuous to Ras 1 and Ras2, which are localized beneath MoMsb2 and MoShol, are non-covalently associated with Mst11 (Li et al., 2012). Existence of $M g b l$, a G $\beta$ subunit, upstream of $P m k l$ is also reported, and apparently acts as a link between cAMP signaling and MAPK pathway (Nishimura et al., 2003; Fernandez and Wilson, 2014a). 


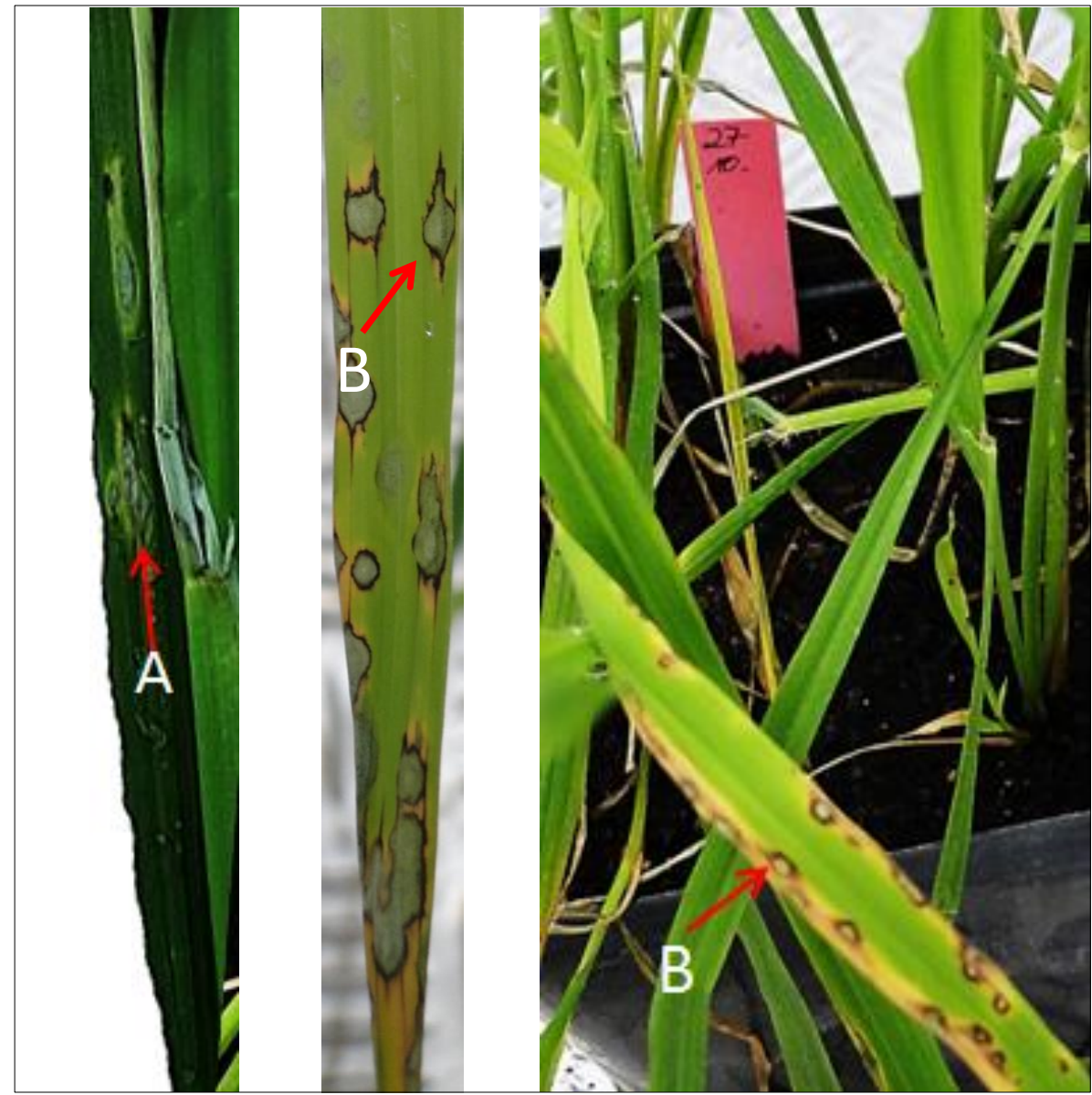

Figure 1.1: Typical rice blast lesions on rice leaf sheath (A) and leaves (B) on 5 weeks grown plants in the greenhouse. The arrows indicate the lesions.

The transcription factor Mst12, a homologue of the yeast STE12, is reported to be a Pmk1 downstream target required for penetration and invasive growth (Park et al., 2002). A MADSbox transcription factor, $M o M c m l$ was recently shown to form a heterodimeric complex with Mst12 in activating components required for infection (Zhou et al., 2011). These multiple events suggest that the infection-related morphogenesis in M. Oryzae is highly regulated, and multiple steps are required for the development of the penetration peg and successful penatration of host tissues.

Differentiation of the infection peg into infection hyphae requires $P L S 1$, which encodes a putative tetraspanin protein (Clergeot et al., 2001). PLS1 is apparently involved in signal transduction essential for reorganization of actin at the base of the appressorium and directing mechanical pressure at the pore (Clergeot et al., 2001; Talbot, 2003). A list of other pathogenicity proteins involved in surface sensing, appressorium formation and the subsequent phases of M. oryzae infection is provided in Figure 1.2. 
Upon plant penetration, and the subsequent invasive growth, $M$. oryzae secretes proteins into the host cell, some of which are categorized as effectors. Effectors are generally defined as pathogen derived proteins and small molecules directed towards manipulation of host cell structure and function, either by facilitating infection (toxins and virulence factors) or by activating defence response (elicitors and avirulence factors) (Kamoun, 2006). About 1306 genes coding for putative secreted proteins have been predicted from $M$. oryzae genome (Zhang and $\mathrm{Xu}, 2014$ ). Effector delivery into host cells is accomplished by fungal hyphal modification into: (1) a biotrophic interfacial complex (BIC), which forms in primary hyphae as they enter each host cell and (2) host-derived extra invasive hyphal membrane (EIHM) surrounding bulbous invasive hyphae (Fernandez and Wilson, 2014a).

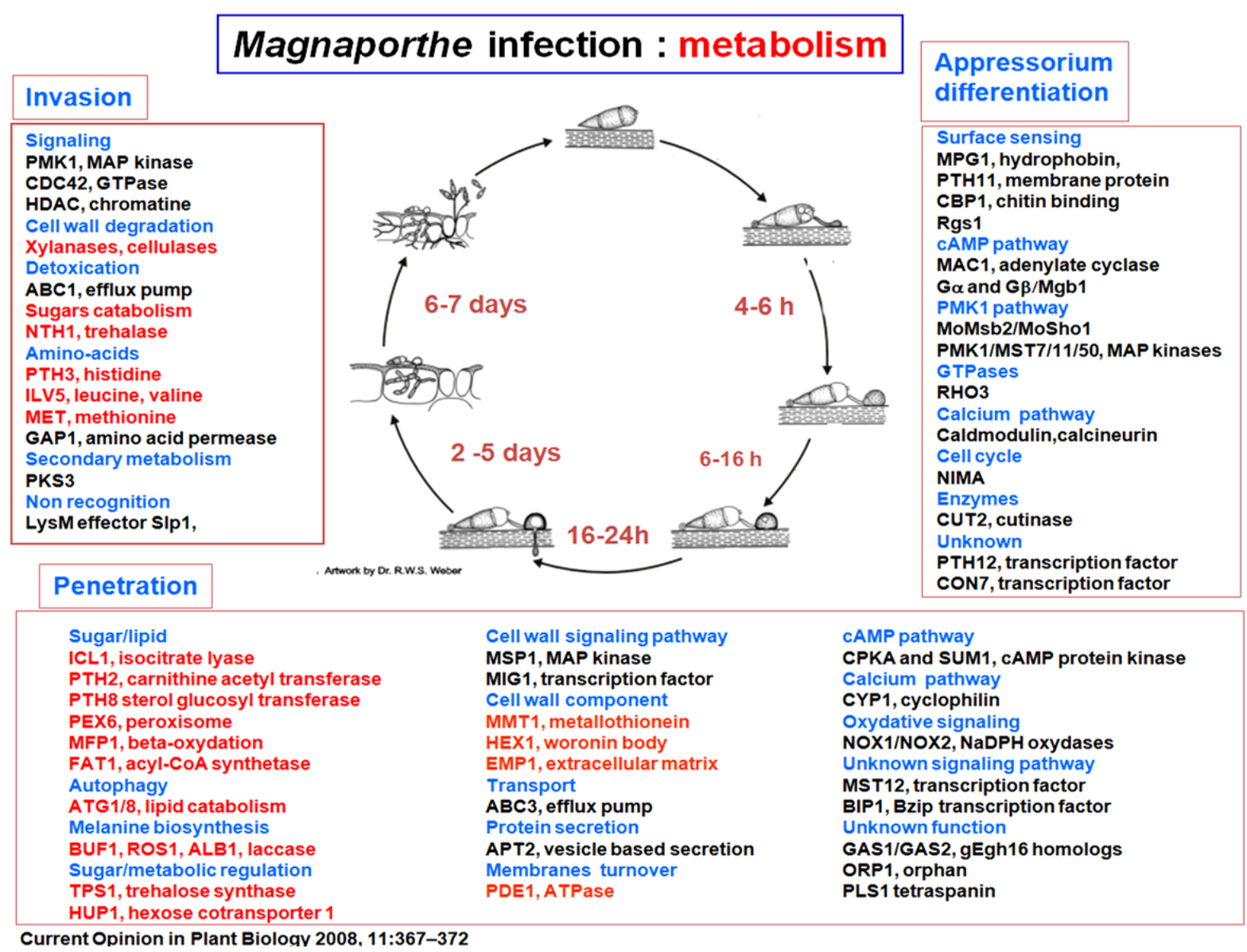

Figure 1.2: Magnaporthe oryzae infection cycle accompanied with pathogenicity proteins involved in each stage; from appressorium differentiation to invasion. Reprinted with permission and support from Marc Henri LEBRUN, INRA-CPP

Two types of effectors are distinguished in $M$. oryzae based on their localization in plant cells: (1) cytoplasmic effectors, including Avr-Pita, PWL1, PWL2, BAS2, and Avr-Piz-t, which accumulate in the BIC before being delivered into plant cells, and (2) apoplastic effectors 
such as BAS4, Avrl-CO39 and Slp1, which are dispersed in the extracellular space between the fungal cell wall and EIHM (Zhang and $\mathrm{Xu}, 2014$ ) where they either interact with plant derived defence proteins or prevent fungal structures from being recognized by the host defences.

Previous reports have shown that apoplastic effectors require the conventional Golgimediated secretion pathway, while cytoplasmic effectors require BIC associated pathway involving the exocyst components Exo70 and Sec5 for their secretion into host cells (Giraldo et al., 2013). However, unlike in oomycetes where cytoplasmic effectors are modular proteins with defined signal peptide motifs for secretion (Schornack et al., 2009), the specific protein motifs or sequences specifying localization in M. oryzae effectors are still elusive. Hence, predicting localization based on amino acid sequence is presently a challenge. Nonetheless, it is hypothesized that effector trafficking may depend on rice cell types, protein size and conserved fungal mechanisms to recognize plasmodesmata for the movement of effector proteins and spreading of invasive hyphae (Zhang and $\mathrm{Xu}, 2014$ ). Validation of these hypotheses will provide more insights into $M$. oryzae infection process.

\section{Variability and population structure studies on M. oryzae}

Magnaporthe oryzae is highly variable with a potential to evolve relatively quickly and adapt to changes in environmental conditions and host resistance (Huang et al., 2014). Genetic variability is correlated with high levels of genetic instability of traits such as pathogenicity, morphology and fertility (reviewed in Scheuermann et al., 2012). Variation in pathogenicity is attributed to genomic alterations that lead to loss of function of avirulence effectors, including point mutations, especially repeat-induced point mutations (Fudal et al., 2009), insertions of transposable elements, or deletions of entire genes (De Wit et al., 2009). Some of the avirulence genes are located in telomeric and sub-telomeric regions, as is the case of Avr-Pita, AvrMedNö̈-1, AvrKu86-1, Avr-Piz, Avr-Pii, Avr-Pi15, Avr-Pit and Avr-Pia (Scheuermann et al., 2012). Deletions in these regions results in the loss of $A v r$ genes, leading to virulence gain (Orbach et al., 2000). As suggested previously, such mutations could also be the major cause of genetic diversity within populations from one region (Tharraeu et al., 2009).

Several molecular typing techniques have been used to understand M. oryzae population structure, including MGR 586 repetitive DNA sequence (Hamer et al., 1989), Pot2 (Kachroo et al., 1994), Randomly amplified polymorphic DNA (RAPD) (Rathour et al., 2004; Sere et 
al., 2007), amplified fragment length polymorphism (AFLP) (Nguyen et al., 2006), simple sequence repeats (SSRs) (Brondani et al., 2000), and pathogenicity tests on differential sets of rice varieties (Levy et al., 1991). These studies have revealed several clonally derived lineages, as well as genetic variability associated with race diversity. Regional clustering of populations has been observed, for instance genotypes from Europe are very similar and are distinct from genotypes from Madagascar, which likewise form one cluster (Tharraeu et al., 2009). Alternatively, evidence has shown that M. oryzae populations are more diverse in Asia, where rice cultivation has been going on for several years, compared to Europe and East Africa where rice cultivation is relatively recent. This is probably due to the abundance of several and diverse rice cultivars and a heterogeneous environment, which prevents clonal expansion of lineages. In the Himalayas and parts of China, where there is high diversity of rice, both mating types of $M$. oryzae are present (Rathour et al., 2004), which suggests that sexual reproduction could be still contributing to genetic diversity in those areas. However, there are suggestions that in some areas where rice cultivation and favorable weather conditions enable the fungus to survive in the form of mycelia during many fallow seasons, mutations that remove sexual reproduction could be fixed because of high energy costs associated with this character (Zeigler, 1998). The fungus, therefore, propagates asexually by means of conidia. Indeed some of the rice growing areas, including USA, Europe, and Africa, have been shown to have near monomorphic populations (Xia et al., 1993; Xia et al., 2000; Roumen et al., 1997; Takan et al., 2011), suggesting that asexual reproduction is a dominant phenomenon in these regions, and that different mechanisms are acting to create genetic variability in $M$. Oryzae. Alternative causes of genetic variation, such as parasexual recombination (Noguchi, 2011) and mutation, could be contributing to genetic diversity observed in such regions. There is, however, no evidence to support these hypotheses, and further studies are warranted.

\section{Management of rice blast}

Rice blast management includes various methods such as chemical, cultural and cultivar resistance or a combination of the three. Chemical control has long been viewed as a last resort to control rice blast. Where necessary, several fungicides have been shown to be effective, including tetrachlorophthalide, tebuconazole + trifloxystobin and propiconazole (Ghazanfar et al., 2009). In cultural control, burning of diseased straw and stubble to minimize carryover of inoculum to the next season crop (Kato, 2001), planting healthy seeds, nitrogen fertilizer management, crop rotation and flooding reduce the amount of inoculum 
available for subsequent disease development. However, host genetic resistance remains a reliable strategy that combines both economic viability and environmental safety aspects.

\section{Genetic resistance to rice blast in rice}

Rice blast resistance is classified into two types: i) complete or true (qualitative) and ii) partial or field resistance (quantitative) (Ezuka 1972). Complete resistance, induced by incompatible interaction, is usually controlled by a major gene, whereas partial resistance is generally nonrace specific and polygenic (Kiyosawa 1981). Partial resistance, also called basal or horizontal disease resistance is sometimes referred to as pathogen-associated molecular pattern (PAMP)-triggered immunity (PTI), and is considered as the primary facet of active plant defence. Major gene resistance, also called effector-triggered immunity (ETI) mostly relies on the endogenious NB-LRR protein products encoded by resistance (R)-genes. Major gene resistance is generally not durable, and usually breaks down a few years after introduction into commercial varieties, whereas partial resistance is preferred because it is thought to lead to durable resistant cultivars (Mackill and Bonman 1992). However, major gene resistance is now effectively being deployed through pyramiding of several R-genes in the same cultivar, which increases resistance durability and spectrum. Moreover, combining several R-genes with horizontal resistance has been hypothesized to be the most promising approach to develop durable resistance in rice (Jena and Mackill 2008).

PTI is mediated by pattern recognition receptors (PRRs) that recognize PAMPs. Generally, PTI and ETI trigger similar defence responses, but ETI is much faster and quantitatively stronger (Wei et al., 2013). In addition, ETI is an efficient defence system for more progressed infections, as it is able to guard against weak points or even setting up decoys to confuse invaders. On the other hand, PTI is important for non-host resistance and for basal immunity in susceptible host plant cultivars. Both PTI and ETI are often activated preceding pathogen recognition. Following pathogen recognition, a cascade of signal transduction pathways, which require regulators such as hydrogen peroxide, salicylic acid (SA), jasmonic acid (JA), ethylene (ET), abscisic acid, mitogen activated protein kinases and transcriptional factors initiate PTI or ETI, depending on the pathogenicity factors recognized by the plant. Subsequently, downstream defence responses which include programmed cell death leading to the hypersensitive response (HR), production of antimicrobial secondary metabolites (e.g., phytoalexins), pathogenesis-related (PR) proteins (e.g., chitinases and glucanases) and cellwall strengthening occur (Vergne et al., 2008). The hallmark of ETI in plant defense is the 
hypersensitive response (HR), which is largely derived from membrane localized NADPH oxidases.

Over 85 blast resistance genes have been identified in rice (Valent and Khang, 2010; Huang et al., 2014), 22 of these have been cloned (Luo et al., 2012). Furthermore, near-isogenic lines with single genes for resistance have been developed in the genetic backgrounds of the $O$. indica variety CO39, O. japonica variety Li-Jiang-Xin-Tuan-He-Gu (LTH) and a universal susceptible line US2 (Kobayashi et al., 2007). These isogenic lines are useful for identification of the blast races, monitoring of shifts in blast races and determining plantspecific resistance in rice breeding (Telebanco-Yanoria et al., 2010; Koide et al., 2011).

Previous studies have reported the molecular processes mediated by $\mathrm{R}$ genes and partial resistance genes during blast infection using differential expression studies: e.g., rice carrying the R gene Pi33 infected with M. oryzae strain carrying the avirulence gene ACE1 (Vergne et al., 2007), resistant vs. susceptible NILs differing for the introgression of the Pi-k gene (Li et al., 2006), Pi54 activation of multiple defense mechanisms in rice (Gupta et al., 2012) and rice early defense response to $M$. oryzae in a compatible and incompatible interaction (Wei et al., 2013). The molecular function of some $\mathrm{R}$ genes in rice defense responses is well documented (Dai et al., 2007; Miah et al., 2010). The functional domain positioning is probably the reason behind several $\mathrm{R}$ genes identified in rice. One of the well-known functional domain positioning examples is the recognition of $M$. oryzae effector, AVR-Piz-t by the $\mathrm{R}$ gene Piz-t, which adopts a six-stranded $\beta$-sandwich structure and contains a single disulphide bond. In addition, the direct binding of AVR-Pik to Pik-1 by interacting with Heavy-Metal Associated domain (HMA; also known as RATX1) of Pik-1 is another domain positioning example that could be different from some $\mathrm{R}$ genes. Thus, the functional domain positioning underlying effector binding could be causing defence-related differences between R genes.

Partial resistance/PTI is conferred by QTLs, and several blast resistance QTLs have been mapped (reviewed in Liu et al., 2010); the genes underlying two of them, pi21 and Pbl, have been cloned (Fukuoka et al., 2009; Hayashi et al., 2010). In addition, transcript profiling of temperate japonica Italian rice cultivars with contrasting response to $M$. oryzae infection using RNA-sequencing (RNA-Seq) was recently carried out in an effort to establish a more meaningful picture of durable resistance in rice (Bagnaresi et al., 2012). These studies 
allowed for the identification of the main biological processes and functional categories of defense related genes responsive to $M$. oryzae in genotypes with contrasting resistance. Alternatively, in a study involving transgenic plants carrying Xa26 (also known as Xa3), it was found that the background of japonica variety Mudanjiang 8 showed increased resistance to eight Xoo strains as compared to the gene donor of indica variety Minghui 63 (Sun et al., 2004). These findings were later confirmed by Cao et al (2007), who found that the genetic background of japonica rice enhanced $\mathrm{Xa3}$ mediated resistance more than the background of indica rice. Genetic characterization studies have also suggested that indica varieties have more complicated blast resistance patterns than japonica varieties (Shang et al., 2009). Moreover, different indica backgrounds appear to have a significant influence on the function of $R$ genes (Cao et al., 2007). Although much is already known about the influence of genetic background on plant response to pathogens, an initial step will be to refine this knowledge further and determine the influence of environmental factors on host genetic backgroundpathogen interaction. In order to address this question, a well characterized pathosystem is required. As reported earlier, the rice-Magnaporthe oryzae pathosystem is an ideal model in this regard.

\section{References}

Bagnaresi, P., Biselli, C., Orrù, L., Urso, S., Crispino, L., Abbruscato, P., Piffanelli, P., Lupotto, E., Cattivelli1, L. and Valè, G. (2012) Comparative transcriptome profiling of the early response to Magnaporthe oryzae in durable resistant vs susceptible rice (Oryza sativa L.) genotypes. PLoS One 7, e51609.

Banniza, S., Sy, A.A., Bridge, P.D., Simons, S.A. and Holderness, M. (1999) Characterization of populations of Rhizoctonia solani in paddy rice fields in Cote d' Ivoire. Phytopathology, 89, 414-420.

Barr, M. (1977) Magnaporthe, Telimenella and Hyponectria (Physosporellaceae). Mycologia, 69, 952-966.

Bokszczanin, K.L., Fragkostefanakis, S., Bostan, H., Bovy, A., Chaturvedi, P., Chiusano, M.L., Firon, N., Iannacone, R., Jegadeesan, S., Klaczynskid, K., Li, H., Mariani, C., Müller, F., Paul, P., Paupiere, M., Pressman, E., Rieu, I., Scharf, K.D., Schleiff, E., Van Heusden, A.W., Vriezen, W., Weckwerth, W. and Winter, P. (2013) Perspectives on deciphering mechanisms underlying plant heat stress response and thermotolerance. Frontiers in Plant Science 4, 315

Bonman, J.M. and MacKill, D.J. (1988) Durable resistance to rice blast disease. Oryza 25, 103-110. 
Brondani, C., Brondani, R., Garrido, L.D. and Ferreira M. (2000) Development of microsatellite markers for genetic analysis of Magnaporthe grisea. Genetics and Mol. Bio. $23,753-762$.

Cannon, P. (1994) The newly recognized family Magnaporthaceae and its interrelationships. Systema Ascomycetum 13, 25-42.

Chen, C., Lian, B., Hu, J., Zhai, H., Wang, X., Venu, R.C., Liu, E., Wang, Z., Chen, M., Wang, B., Wang, G.L., Wang, Z. and Mitchell, T.K. (2013) Genome comparison of two Magnaporthe oryzae field isolates reveals genome variations and potential virulence effectors. BMC Genomics 14, 887.

Clergeot, P.H., Gourges, M., Cots, J., Laurans, F., Latorse, M.P., et al., (2001) PLS1, a gene encoding a tetraspanin-like protein, is required for penetration of rice leaf by the fungal pathogen Magnaporthe grisea. Proc Natl Acad Sci USA 98, 6963-6968.

Couch, B.C. and Cohn, L.M. (2002) A multilocus gene genealogy concordant with host preference indicates segregation of a new species, Magnaporthe oryzae from M. grisea. Mycologia 94, 683-693.

Couch, B.C., Fudal, I., Lebrun, M.H., Tharreau, D., Valent, B., van Kim, P., Notteghem, J.L., et al., (2005) Origins of host-specific populations of the blast pathogen Magnaporthe oryzae in crop domestication with subsequent expansion of pandemic clones on rice and weeds of rice. Genetics 170, 613-630.

Dai, Y., Jia, Y., Correll, J.C., Wang, X. and Wang, Y. (2010) Diversification and evolution of the avirulence gene ARV-Pital in field isolates of Magnaporthe oryzae. Fungal Genet Biol 47, 973e980.

Dean, R.A., Talbot, N.J., Ebbole, D.J., Farman, M.L., Mitchell, T.K., Orbach, M.J., Thon, M., Kulkarni, R., Xu, J.R., Pan, H.Q., Read, N.D., Lee, Y.H., Carbone, I., Brown, D., Oh, Y.Y., Donofrio, N., Jeong, J.S., Soanes, D.M., Djonovic, S., Kolomiets, E., Rehmeyer, C., Li, W.X., Harding, M., Kim, S., Lebrun, M.H., Bohnert, H., Coughlan, S., Butler, J., Calvo, S., Ma, L.J., Nicol, R., Purcell, S., Nusbaum, C., Galagan, J.E., and Birren, B.W. (2005) The genome sequence of the rice blast fungus Magnaporthe grisea. Nature 434, 980-986.

DeWit, P.J.G.M., Mehrabi, R., van den Burg, H. and Stergiopoulos, I. (2009) Fungal effector proteins: past, present and future. Molecular Plant Pathology 10, 735-747.

DeZwaan, T.M., Carroll, A.M., Valent, B. and Sweigard, J.A. (1999) Magnaporthe grisea Pth11p is a novel plasma membrane protein that mediates appressorium differentiation in response to inductive substrate cues. Plant Cell 11, 2013-2030.

Ezuka, A. (1972) Field resistance of rice varieties to rice blast disease. Rev Plant Prot Res $5,1-21$ 
Fernandez, J. and Wilson, R.A. (2014a) Cells in cells: morphogenetic and metabolic strategies conditioning rice infection by the blast fungus Magnaporthe oryzae. PLoS ONE 9, e87300.

Fudal, I., Collemare, J., Bohnert, H.U., Melayah, D. and Lebrun, M.H. (2007) Expression of Magnaporthe grisea avirulence gene ACE1 is connected to the initiation of appressorium-mediated penetration. Eukaryotic cell 6 no 3, 546.

Fukuoka, S., Saka, N., Koga, H., Ono, K., Shimizu T., et al., (2009) Loss of function of a proline-containing protein confers durable disease resistance in rice. Science 325, 9981001.

Fukuoka, S., Saka, N., Koga, H., Ono, K., Shimizu, T., Ebana, K., Hayashi, N., Takahashi, A., Hirochika, H., Okuno, K. and Yano, M. (2009) Loss of function of a proline-containing protein confers durable disease resistance in rice. Science 325, 9981001.

Garber, M., Grabherr, M.G., Guttman, M. and Trapnell, C. (2011) Computational methods for transcriptome annotation and quantification using RNA-seq. Nature Methods 8, 469-477.

Ghazanfar, M.U., Wakil, W., Sahi, S.T. and Saleem-il-Yasin. (2009). Influence of various fungicides on the management of rice blast disease. Mycopath. 7(1), 29-34.

Giraldo, M.C., Dagdas, Y.F., Gupta, Y.K., Mentlak, T.A., Yi, M., et al., (2013) Two distinct secretion systems facilitate tissue invasion by the rice blast fungus Magnaporthe oryzae. Nat Commun 4, 1996.

Gousseau, H.D.M., Deverall, B.J. and Mclntosh, R.A. (1985) Temperature-sensitivity of the expression of resistance to Puccinia graminis conferred by the Srl5, SrVh and Srl4 genes in wheat. Physiological Plant Pathology 27, 33543.

Greer, C.A. and Webster, R.K. (2001) Occurrence, Distribution, Epidemiology, Cultivar Reaction, and Management of Rice Blast Disease in California. Plant Dis 85, 1096-1102.

Gupta, S. K., Rai, A.K., Kanwar, S.S., Chand, D., Singh, N. K., et al., (2012) The single functional blast resistance gene Pi54 activates a complex defence mechanism in rice. J Exp Bot 63, 757-772.

Hamer, J., Farral, L., Orbach, M., Valent, B. and Chumley, F.G. (1989) Host speciesspecific conservation of a family of repeated DNA sequences in the genome of a fungal plant pathogen. Proceedings of the National Academy of Sciences of the United States of America 86, 9981-9985.

Hayashi, N., Inoue, H., Kato, T., Funao, T., Shirota, M., et al., (2010) Durable panicle blast-resistance gene $\mathrm{Pb} 1$ encodes an atypical CC-NBS-LRR protein and was generated by acquiring a promoter through local genome duplication. The Plant Journal 64, 498-510. 
Hayashi, N., Inoue, H., Kato, T., Funao, T., Shirota, M., Shimizu, T., Kanamori, H., Yamane, H., Hayano-Saito, Y., Matsumoto, T., Yano, M. and Takatsuji, H. (2010) Durable panicle blast-resistance gene $\mathrm{Pb} 1$ encodes an atypical CC-NBS-LRR protein and was generated by acquiring a promoter through local genome duplication. Plant J 64, 498510 .

Hepworth, N., and Goulden, M. (2008) Climate change in Uganda: Understanding the implication and appraising the response. LTS International Edinburgh.

Howard, R. and Valent B. (1996) Breaking and entering: Host penetration by fungal rice blast pathogen Magnaporthe grisea. Annual Review of Microbiology 50, 491-512.

Huang, J., Si, W., Deng, Q., Li, P. and Yang, S. (2014) Rapid evolution of avirulence genes in rice blast fungus Magnaporthe oryzae. BMC Genet 15, 45.

International Rice Genome Sequencing Project (IRGSP). (2005) The map-based sequence of the rice genome. Nature 436,793-800.

IPCC: Intergovernmental Panel on Climate Change. (2007) Climate change 2007: The physical science basis. Summary for policymakers. Contribution of working group I and II to the third assessment report of the IPCC. IPCC Secretariat, Geneva, Switzerland.

Jena, K.K. and Mackill, D.J. (2008) Molecular markers and their use in marker-assisted selection in rice. Crop Sci 48, 1266-1276.

Jorgensen, T.H. (2012) The effect of environmental heterogeneity on RPW8-mediated resistance to powdery mildews in Arabidopsis thaliana. Ann Bot 109, 833-842.

Wu, J., Kou, Y., Bao, J., Li, Y., Tang, M., Zhu, X., Ponaya, A., Xia, G., Li, J., Li, C., Song, M., Joseph, C., Deng, Q., Lu, G., Jeon, J., Naqvi, N. and Zhou, B. (2015): Comparative genomics identifies the Magnaporthe oryzae avirulence effector AvrPi9 that triggers Pi9-mediated blast resistance in rice. New phytologist 206, 1463-1475.

Juroszek, P. and von Tiedemann, A. (2012) Climate change and potential future risks through wheat diseases. Eur J Plant Pathol 36, 21-33.

Kachroo, P., Leong, S. A. and Chattoo, B. B. (1995) Mg-SINE: a short interspersed nuclear element from the rice blast fungus, Magnaporthe grisea. Proc Natl Acad Sci USA 92, 11125-11129.

Kachroo, P., Leong, S. and Chattoo, B. (1994) Pot2, an inverted repeat transposon from the rice blast fungus Magnaporthe grisea. Molecular and General Genetics 245(3), 339-348.

Kamoun, S. (2006) A catalogue of the effector secretome of plant pathogenic oomycetes. Annu Rev Phytopathol 44, 41-60.

Kato, H. (2001). Rice blast disease - An Introduction. Pesticide Outlook, 23-25.

Kato, H. and Kozaka, T. (1974) Effects of temperature on lesion enlargement and sporulation of Pyricularia oryzae in rice leaves. Phytopathology 64, 828-830. 
Kato, H., Yamamoto, M., Yamaguchi-Ozaki, T., Kadouchi, H., Iwamoto, Y., Nakayashiki, H., Tosa,Y., Mayama, S. and Mori N. (2000) Pathogenicity, mating ability and DNA restriction fragment length polymorphisms of Pyricularia populations isolated from Gramineae, Bambusideae and Zingiberaceae. Plants Journal General Plant Pathology 66(1), 30-47.

Kawahara, Y., Oono, Y., Kanamori, H., Matsumoto, T., Itoh, T. and Minami, E. (2012) Simultaneous RNA-Seq analysis of a mixed transcriptome of rice and blast fungus interaction. PloS One 7, e49423.

Kihoro, J., Njoroge, J. B., Murage, H., Ateka, E. and Makihara, D. (2013) Investigating the impact of rice blast disease on the livelihood of the local farmers in greater Mwea region of Kenya. SpringerPlus 2, 308

Kiyosawa, S. (1981) Gene analysis for blast resistance. Oryza 18, 196-203.

Kobayashi, N., Telebanco-Yanoria, M.J., Tsunematsu, H., Kato, H., Imbe, T. and Fukuta, Y. (2007) Development of new sets of international standard differential varieties for blast resistance in rice (Oryza sativa L.). JARQ 41, 31-37.

Koide,Y., Telebanco-Yanoria, M.J., Peña, F.D., Fukuta, Y. and Kobayashi, N. (2011) Characterization of rice blast isolates by the differential system and their application for mapping a resistance gene. Pi19 (t) J Phytopathol 159, 85e93.

Laine, A.L. (2007) Pathogen fitness components and genotypes differ in their sensitivity to nutrient and temperature variation in a wild plant-pathogen association. Journal of Evolutionary Biology 20, 2371-2378.

Leung, H., Borromeo S., Bernardo, M. A. and Notteghem, J. L. (1988) Genetic analysis of virulence in the rice blast fungus Magnaporthe grisea. Phytopathol 78, 1227-1233.

Levy, M., Romao, J., Marchetti, M. A. and Hamer, J. E. (1991) DNA fingerprinting with dispersed repeated sequence resolves pathotype diversity in the rice blast fungus. Plant Cell 3, 95-102.

Li, Q., Chen, F., Sun, L., Zhang, Z., Yang, Y., et al., (2006) Expression profiling of rice genes in early defence responses to blast and bacterial blight pathogens using cDNA microarray. Physiological and Molecular Plant Pathology 68, 51-60.

Li,G., Zhou, X. and Xu, J.R. (2012) Genetic control of infection-related development in Magnaporthe oryzae. Curr Opin Microbiol 15, 678-684.

Liu, H., Suresh, A., Willard, F.S., Siderovski, D.P., Lu, S., et al., (2007) Rgs1 regulates multiple $G$ alpha subunits in Magnaporthe pathogenesis, asexual growth and thigmotropism. Embo Journal 26, 690-700.

Liu, J., Wang, X., Mitchell, T., Hu, Y., Liu, X., Dai, L. and Wang, G.L. (2010) Recent progress and understanding of the molecular mechanisms of the rice-Magnaporthe oryzae interaction. Molecular Plant Pathology 11, 419-427. 
Liu, W., Zhou, X., Li, G., Li, L., Kong, L., et al., (2011) Multiple plant surface signals are sensed by different mechanisms in the rice blast fungus for appressorium formation. PLoS Pathog 7, e1001261.

Luo, S., Zhang, Y., Hu, Q., Chen, J., Li, K., Lu, C., Liu, H., Wang, W. and Kuang, H. (2012) Dynamic nucleotide-binding site and leucine-rich repeat-encoding genes in the grass family. Plant Physiol 159, 197-210.

Luo, Y., Teng, P.S., Fabellar, N.G. and TeBeest, D.O. (1998) The effects of global temperature change on rice leaf blast epidemics: a simulation study in three agroecological zones. Agriculture Ecosystems and Environment 68, 187-196.

Mackill, D.J. and Bonman, J.M. (1992) Inheritance of blast resistance in near-isogenic lines of rice. Phytopathology 82, 746-749.

Miah, G., Rafii, M.Y., Ismail, M.R., Puteh, A.B., Rahim, H.A., Asfaliza, R. and Latif, M.A. (2013) Blast resistance in rice: a review of conventional breeding to molecular approaches. Mol Biol Rep. 40, 2369-2388.

Nguyen, T., Bigirimana, J., Roumen, E., Van Der Straeten, D. and Hofte, M. (2006) Molecular and pathotype analysis of the rice blast fungus in North Vietnam. European Journal of Plant Pathology 114, 381-396.

Nishimura, M., Park, G. and Xu, J.R. (2003) The G-beta subunit MGB1 is involved in regulating multiple steps of infection-related morphogenesis in Magnaporthe grisea. Mol Microbiol 50, 231-243.

Noguchi, M. (2011) Parasexual recombination in Magnaporthe oryzae. Japan Agricultural Research Quarterly 45(1), 39-45.

Orbach, M., Farrall, L., Sweigard, J., Chumley, F. and Valent, B. (2000) A Telomeric avirulence gene determines efficacy for the rice blast resistance gene Pi-ta. The Plant Cell 12(4), 2019-2032.

Ou, S.H. (1985) Rice Diseases, 2nd ed. Kew, Surrey, U.K. Commonwealth Mycological Institute.

Palloix, A, Ayme, V and Moury, B. (2009) Durability of plant major resistance genes to pathogens depends on the genetic background, experimental evidence and consequences for breeding strategies. New Phytol. 183, 190-199.

Pangga, I.B., Hanan, J. and Chakraborty, S. (2011) Pathogen dynamics in a crop canopy and their evolution under changing climate. Plant Pathology 60, 70-81.

Park, G., Xue, G.Y., Zheng, L., Lam, S. and Xu, J.R. (2002) MST12 regulates infectious growth but not appressorium formation in the rice blast fungus Magnaporthe grisea. Mol Plant Microbe Interact 15, 183-192. 
Piotti, E., Rigano, M.M., Rodino, D., Rodolfi, M., Castiglione, S., Picco, A.M. and Sala, F. (2005) Genetic Structure of Pyricularia grisea (Cooke) Sacc. Isolates from Italian Paddy Fields. J. Phytopathol 153, 80-86.

Rathour, R., Singh, B., Sharma, T. and Chauhan, R. (2004) Population Structure of Magnaporthe grisea from North-western Himalayas and its implications for blast resistance breeding of rice. Journal of Phytopathology 152(5), 304-312.

Scheuermann, K.K., Raimondi, J.V., Marschalek, R., de Andrade, A. and Wickert, E. (2012) Magnaporthe oryzae genetic diversity and its outcomes on the search for durable resistance. In Caliskan, M. (Ed.), The molecular basis of plant genetic diversity (pp. 331356). InTech.

Schornack, S., Huitema, E., Cano, L.M., Bozkurt, T.O., Oliva, R., et al., (2009) Ten things to know about oomycete effectors. Mol Plant Pathol 10, 795-803.

Séré, Y., Onasanya, A., Afolabi, A., Mignouna, H. and Akator, K. (2007) Genetic diversity of the blast fungus, Magnaporthe grisea (Hebert) Barr, in Burkina Faso. African Journal of Biotechnology 6(22), 2568-2577.

Sesma, A. and Osbourn, A. (2004). The rice leaf blast pathogen undergoes developmental processes typical of root-infecting fungi. Nature 431, 582-586.

Shang, J., Tao, Y., Chen, X., Zou, Y., Lei, C., Wang, J., Li, X., Zhao, X., Zhang, M., Lu, Z., Xu, J., Cheng, Z., Wan, J. and Zhu, L. (2009) Identification of a new rice blast resistance gene, Pid3, by genome-wide comparison of paired NBS-LRR genes and their pseudogene alleles between the two sequenced rice genomes. Genetics, 182, 1303-1311.

Soanes, D.M., Chakrabarti, A., Paszkiewicz, K.H., Dawe, A.L. and Talbot, N.J. (2012) Genome-wide transcriptional profiling of appressorium development by the rice blast fungus Magnaporthe oryzae. PLoS Pathog 8, e1002514.

Talbot, N.J. (2003) On the trail of a cereal killer: exploring the biology of Magnaporthe grisea. [Review] Annu Rev Microbiol 57, 177-202.

Telebanco-Yanoria, M.J., Koide, Y., Fukuta, Y., Imbe, T., Kato, H., Tsunematsu, H. and Kobayashi, N. 2010. Development of near-isogenic lines of Japonica-type rice variety Lijiangxintuanheigu as differentials for blast resistance. Breed Sci 60, 629e638.

Tharraeu, D., Fudal, I., Andriantsimialona, D., Santoso, Utami, D., Fournier, E., Lebrun M-H. and Nottéghem J.L. (2009) World Population Structure and Migration of the Rice Blast Fungus, Magnaporthe oryzae. Advances in Genetics, Genomics and Control of Rice Blast Disease Part III, 209-215.

Urashima, A.S. (2001) Variation in virulence in the rice blast fungus Magnaporthe grisea in São Paulo State. Pesq agropec bras Brasília37, 109-115.

Valent, B. and Khang, C.H. (2010) Recent advances in rice blast effector research. Current Opinion in Plant Biology 13, 434-441. 
Vergne, E, Ballini E, Marques S, Mammar BS, Droc G, Gaillard S, Bourot S, DeRose R, Tharreau D, Notteghem J-L et al., 2007. Early and specific gene expression triggered by rice resistance gene Pi33 in response to infection by ACE1 avirulent blast fungus. New Phytologist 174, 159-171.

Vergne, E., Grand, X., Ballini, E., Chalvon, V., Saindrenan, P., Tharreau, D., Nottéghem, J.L., Morel, J.B., et al., (2010) Preformed expression of defence is a hallmark of partial resistance to rice blast fungal pathogen Magnaporthe oryzae. BMC Plant Biology 10, 206.

Wang, Z.Y., Jenkinson, J., Holcombe, L., Soanes, D., Veneault-Fourrey, C. and Bhambra, G. (2005) The molecular biology of appressorium turgor generation by the rice blast fungus Magnaporthe grisea. Biochemical Society Transactions 33 part 2, 384-388.

Wei, T., Ou, B., Li, J., Zhao, Y., Guo, D., et al., (2013). Transcriptional Profiling of Rice Early Response to Magnaporthe oryzae Identified OsWRKYs as Important Regulators in Rice Blast Resistance. PLoS ONE 8, e59720.

Wilson, R.A. and Talbot, N.J. (2009) Under pressure: investigating the biology of plant infection by Magnaporthe oryzae. Nat Rev Microbiol 7, 185-195.

Xue, M.F., Yang, J., Li, Z.G., Hu, S.N.A., Yao, N., Dean, R.A., Zhao, W.S., Shen, M., Zhang, H.W., Li, C., et al., (2012) Comparative analysis of the genomes of two field isolates of the rice blast fungus Magnaporthe oryzae. PLoS Genet 8, e1002869.

Yoshida, K., Saitoh, H., Fujisawa, S., Kanzaki, H., Matsumura, H., Tosa, Y., Chuma, I., Takano, Y., Win, J., Kamoun, S., et al., (2009) Association genetics reveals three novel avirulence genes from the rice blast fungal pathogen Magnaporthe oryzae. Plant Cell 21(5), 1573-1591.

Zeigler, R. (1998) Recombination in Magnaporthe grisea. Annual Reviews of Phytopathology 36, 249-275.

Zhang, S. and Xu, J.R. (2014) Effectors and Effector Delivery in Magnaporthe oryzae. PLoS Pathog 10, e1003826.

Zhou, X., Liu, W., Wang, C., Xu, Q., Wang, Y., Ding, S. and Xu, J. R. (2011) A MADSbox transcription factor $\mathrm{MoMcm} 1$ is required for male fertility, microconidium production and virulence in Magnaporthe oryzae. Mol Microbiol 80, 33-53. 
Chapter 2: Rice NILs carrying Pi54 in LTH and Co39 backgrounds confer resistance to Magnaporthe oryzae at $35^{\circ} \mathrm{C}$ but show contrasting reactions at $28^{\circ} \mathrm{C}^{*}$

*) Parts of this chapter have been submitted for publication in: Onaga, G., Wydra, K.., Koopmann, B., Séré, Y. and von Tiedemann, A. (2016) High temperature effects on Pi54 conferred resistance to Magnaporthe oryzae in two genetic backgrounds of Oryza sativa

.First, third and last authors: Division of Plant Pathology and Crop Protection, Department of Crop Sciences, Georg-August-University Göttingen, Germany; second author: Erfurt University of Applied Sciences, Horticulture - Plant Production and Climate Change, Germany; fourth author: Africa Rice Center, P.O Box 33581, Dar es Salaam, Tanzania.

\section{Introduction}

Global climate models project an increase in average global temperatures by 2 to $5^{\circ} \mathrm{C}$ until the end of this century (Bokszczanin and Fragkostefanakis, 2013). In the past three decades the average global surface temperatures were reported to have increased by $0.2^{\circ} \mathrm{C}$ per decade, and eleven out of twelve years registered as the warmest in history since 1850, occurred between 1995 and 2006 (Ghini et al., 2008). Knowledge on the mechanisms underlying the effect of high temperature (HT) on plant pathogen interaction is however limited, and has been largely contrasting. For instance, HT suppressed the resistance of wheat to Puccinia graminis tritici (Harder et al., 1979) and the resistance of soybean to Phytophthora sojae (Gijzen et al., 1996). The amount of EDS1 and PAD4 transcripts was also reported to be temperature dependent in Arabidopsis, with lower accumulation at $28^{\circ} \mathrm{C}$ than at $22^{\circ} \mathrm{C}$ (Yang and Hua, 2004). Conversely, the yellow rust resistance gene $\operatorname{Yr} 36$ was reported to be up-regulated at HT, leading to full or partial resistance against stripe rust (Puccinia striiformis) in spring wheat (Fu et al., 2009). High temperature also positively modulated $X a 7$ and Rp1-D21 mediated resistance in rice (Webb et al., 2010) and maize (Negeri et al., 2013), respectively. Thus, plant response to both HT and pathogen stress, when occurring simultaneously, can be varied in multiple ways.

Magnaporthe oryzae is the most destructive pathogen of rice, a staple crop for over half of the world's population (Xue et al. 2012). The primary breeding tool in rice blast management is to introduce resistance $(\mathrm{R})$ genes into elite rice cultivars. During the past 25 years, genetic and molecular analysis of resistant donors has led to identification and mapping of more than 100 major blast resistance (R) genes; 22 of these genes have been cloned and used for crop protection against $M$. oryzae (Zhang et al., 2015). Some of these $\mathrm{R}$ genes have been reported 
to confer broad-spectrum resistance against $M$. oryzae strains tested under normal growth conditions (Liu et al., 2002; Jiang et al., 2012). However, the potential of rice and the introgressed R genes to adapt to both HT and M. oryzae stresses remains to be elucidated.

In the present study two rice NILs having Co39 (Oryzae sativa-indica, CO) and LTH (O. sativa-japonica, LT) as genetic backgrounds, and carrying the R gene (Pi54) were used to determine the response of rice to HT and Mo infection. Pi54 possesses a nucleotide binding site-leucine rich repeat (NBS-LRR) domain in addition to a small zinc finger domain, and was previously reported to be constitutively expressed at a basal level in both resistant transgenic as well as susceptible rice lines up to 48 hpi (Gupta et al., 2012).

\section{Results}

\section{Phenotypic performance of infected plants after high temperature exposure}

We investigated the interaction between rice and $M$. oryzae under the normal growth temperature of $28^{\circ} \mathrm{C}$ and a warmer temperature of $35^{\circ} \mathrm{C}$. Two rice genotypes, Li-Jiang-XinTuan-He-Gu (LT; Oryza japonica) and Co39 (CO; Oryza indica) carrying Pi54 R gene, were used. At $28^{\circ} \mathrm{C}, \mathrm{CO}$ had a severe disease phenotype and displayed lesions within 5 days post inoculation (dpi), while LT exhibited reduced lesion sizes (Figure 2.1a). Pi54-associated lesion development was rarely observed in the plants grown and inoculated at $35^{\circ} \mathrm{C}$. To exempt any direct effect of $\mathrm{HT}$ on the pathogen, such plants were grown at $35^{\circ} \mathrm{C}$ for $7 \mathrm{~d}$ prior to Mo inoculation, where after all procedures were carried out at identical temperature conditions $\left(28^{\circ} \mathrm{C}\right)$. Pre-inoculation exposure to $35^{\circ} \mathrm{C}$ induced a delay in disease progress and typical HR lesions in both NILs (Figure 2.1b). To further investigate HT modulation of plant defence, the level of callose deposition in plants subjected to $35^{\circ} \mathrm{C}$ and inoculated with Mo was compared with that in plants grown and inoculated at $28^{\circ} \mathrm{C}$. Ample callose deposition was observed in LT than $\mathrm{CO}$ at $28^{\circ} \mathrm{C}$. At $35^{\circ} \mathrm{C}$, a stronger induction of callose accumulation and intense cell wall fluorescence of the attacked epidermal cells was detected in both NILs (Figure 2.2).

\section{Transcription profiles of $\mathrm{CO}$ and $\mathrm{LT}$ infected with $M$. oryzae at $28^{\circ} \mathrm{C}$ and $35^{\circ} \mathrm{C}$}

To determine temperature-induced transcriptome changes, genome-wide gene expression patterns were investigated in CO and LT using mRNA-Seq following exposure of rice plants to $35^{\circ} \mathrm{C}$ and Mo. An overview of the sequential steps from sample collection to data analysis 
is provided in Figure 2.3a. Three biological replicates of each treatment were sequenced, yielding an average of 69,315,066 single-end reads. More than $80 \%$ of the total reads reached the quality scores at Q20 (Phred score), although a reduced number of quality reads was found in plants treated with double stress involving high temperature and $M$. oryzae infection (Figure 2.3b). To obtain the expression profiles and predict gene structures, the sequenced reads were mapped against the Ensembl cDNA release 16 for the Oryza sativa genome. Using Samtools generated BAM files as input, the R/Bioconductor environment was employed to calculate gene expression levels. We included both unique and multireads in the analysis to avoid undercounting genes with closely related paralogs. A false discovery rate (FDR) of 0.05 was set for the calling of differentially expressed genes (DEGs). The number of DEGs in infected rice $\left(\mathrm{CO}+28^{\circ} \mathrm{C}+\mathrm{Mo}, \mathrm{LT}+28^{\circ} \mathrm{C}+\mathrm{Mo}, \mathrm{CO}+35^{\circ} \mathrm{C}+\mathrm{Mo} \mathrm{LT}+35^{\circ} \mathrm{C}+\mathrm{Mo}\right)$ was significantly higher than in rice plants subjected to only high temperature $\left(\mathrm{CO}+35^{\circ} \mathrm{C}\right.$ and $\left.\mathrm{LT}+35^{\circ} \mathrm{C}\right)$ (Additional file S1a-f). A considerable number of significantly regulated genes was shared by all the infected rice samples $(3,557$ genes), irrespective of the temperature treatment (Figure 2.4a). A total of 600 significantly regulated genes were shared between high temperature stress and M. oryzae infection (Additional file S2). A large number of DEGs $(2,394)$ was shared between LT and CO in response to high temperature (Figure 2.4b). From the upregulated temperature responding genes, 3,459 were exclusive to LT, 750 were found only in $\mathrm{CO}$, and 969 were identified in both genotypes. From the down-regulated genes, 1,778 and 737 were identified as LT and CO-specific, respectively, and 1,400 were repressed in both genotypes. Hierarchical clustering analysis on the $\log 2$ transformed fold change values showed substantial transcriptional profile variation (Figure $2.4 \mathrm{c}$ ). The response to only high temperature was distinct, and clustered separately, from infected samples.

\section{Gene ontology enrichment analysis of DEGs from infected samples at both temperatures}

Gene ontology (GO) categories were assigned to significant DEGs using the goseq $\mathrm{R}$ package, which requires the input of gene lengths based on the median length of transcripts associated with each locus (Young et al., 2010). We used the DEG estimates from DESeq analysis as input files for goseq. We found 34, 37, 35 and $30 \mathrm{GO}$ terms overrepresented in $\mathrm{CO}+28^{\circ} \mathrm{C}+\mathrm{Mo}, \mathrm{CO}+35^{\circ} \mathrm{C}+\mathrm{Mo}, \mathrm{LT}+28^{\circ} \mathrm{C}+\mathrm{Mo}$ and $\mathrm{LT}+35^{\circ} \mathrm{C}+\mathrm{Mo}$, respectively, with more than 4-fold increased enrichment value at a FDR value of $\geq 0.05$ (Figure 2.5, Additional file S3a-d). The GO terms: defense response to the fungus, incompatible interaction (GO:0009817) and flavonoid biosynthetic process (GO:0009813) were unique to 
$\mathrm{CO}+35^{\circ} \mathrm{C}+\mathrm{Mo}, \mathrm{LT}+28^{\circ} \mathrm{C}+\mathrm{Mo}$ and $\mathrm{LT}+35^{\circ} \mathrm{C}+\mathrm{Mo}$. Response to biotic stimulus (GO:0009607) and heat acclimation (GO:0010286) were more enriched in LT than in CO. Diterpene phytoalexin metabolic process (GO:0051501), carbon utilization (GO:0015976) and cellular response to calcium ions (GO:0071277) were common to both backgrounds. When we analyzed only gene sets that are up-regulated in $\mathrm{CO}+35^{\circ} \mathrm{C}+\mathrm{Mo}$ and $\mathrm{LT}+35^{\circ} \mathrm{C}+\mathrm{Mo}$, oxidationreduction process was consistently significant in both backgrounds (Figure 2.6). However, cellular metabolic process, protein ubiquitination, carbon utilization, and response to salicylic acid stimulus were more enriched in $\mathrm{CO}+35^{\circ} \mathrm{C}+\mathrm{Mo}$, whereas response to salt stress, transmembrane transport, response to cadmium ion, cilliary or flagella motility, response to bacterium and cellular protein metabolic process were significantly enriched in $\mathrm{LT}+35^{\circ} \mathrm{C}+\mathrm{Mo}$. Down-regulated genes in both genetic backgrounds were mainly involved in photosynthesis (GO:0009765), citrate transport (GO:0015746) and chlorophyll biosynthetic process (GO:0015995). Our data show that many different pathways were involved and critical processes for the protection of the plant from stress such as oxidation-reduction process were highly enriched.

\section{Gene expression profiles in $\mathrm{LT}$ and $\mathrm{CO}$ in response to only high temperature $\left(35^{\circ} \mathrm{C}\right)$}

Heat stress presents an added degree of complexity in plant defense response to pathogen attack, as the response to heat is apparently controlled by largely different signaling pathways, and may interact positively or negatively with pathogen defense. We observed a significant overlap between genes induced and repressed by heat stress and genes induced by pathogen infection (Figure 2.4c). Since temperature-induced resistance to $M$. oryzae was more pronounced in $\mathrm{CO}$ than in LT, we studied high temperature responding genes and enriched GO terms in $\mathrm{CO}$ in contrast to LT. We found 145 significantly enriched GO terms $(\mathrm{FDR} \leq 0.05)$ in CO compared to 7 in LT (Table S1). The GO categories that were specifically enriched in $\mathrm{CO}$ in response to high temperature included starch biosynthetic process (GO:0019252), sucrose transport (GO:0015770), phosphatidylinositol metabolic process (GO:0046488) and galactolipid biosynthetic process (GO:0019375). GO analysis of LT DEGs revealed significant enrichment of pseudouridine synthesis (GO:0001522), RNA modification (GO:0009451) and flavonoid biosynthetic process (GO:0009813). Oxidation-reduction process (GO:0055114), zinc ion homeostasis (GO:0055069) and phenylpropanoid metabolic process (GO:0009698) were enriched in both backgrounds (Figure S1). 
Among the defense response genes up-regulated in response to only high temperature, NBARC (LOC_Os11g45190) and PR6 (RBBI2-0; LOC_Os01g03680) were >34-fold induced in both backgrounds (Figure 2.7). Some NBS-LRR genes (e.g., LOC_Os11g45180, LOC_Os11g44960 and LOC_Os11g45050) were specifically induced in CO, whereas Pit (LOC_Os01g05620), NBS-LRR (LOC_Os01g05600) which shares 92\% sequence homology with Pit and a putative stripe rust resistance protein Yr10 homolog (LOC_Os12g17090) were induced in LT but not in CO. In the GO term, response to heat GO:0009408, twenty two transcripts were induced in LT compared to only six in CO. Genes encoding for small HSPs e.g., HSP16.9A (LOC_Os01g04370) were highly expressed in LT, whereas LOC_Os08g26230 and a heat shock protein 83 (HSP90; LOC_Os04g01740) exhibited higher induction in CO than in LT. Sequence alignment of LOC_Os08g26230 using Beijing Genomics Institute Rice Information System (BGI-RIS) revealed a 94\% identity to BGIOSGA000853 with Serine/threonine-protein kinase activity. Several genes in the GO term, response to oxidative stress, showed contrasting expression patterns. For example, some peroxidases (e.g., LOC_Os04g59200 and LOC_Os08g41090), oxidoreductases (e.g., aldo/keto reductase; LOC_Os10g02490) and metallothionein-like protein 3B (e.g., LOC_Os05g11320) were strongly induced in LT than CO, whereas peroxidases (e.g., LOC_Os03g55410 and LOC_Os06g20150) were more induced in CO than in LT. Genes encoding for cytochrome P450 (e.g., LOC_Os01g36294) and CASP-like protein (LOC_Os03g60250) were induced by high temperature stress in both genetic backgrounds. In the GO term "response to hormone stimulus", SAM dependent carboxyl methyltransferase (LOC_Os06g13310), OsGH3.2 (LOC_Os01g55940), 2OG-Fe (II) oxygenase domain containing protein (LOC_Os06g07914) were specifically up-regulated in CO but not in LT. Genes encoding for cytokinin-O-glucosyltransferase (e.g., LOC_Os02g51930) were strongly induced in both backgrounds. A transcription factor OsIAA13 - Auxin-responsive Aux/IAA gene family member (LOC_Os03g53150) and C2H2 zinc finger proteins (LOC_Os06g34400) involved in zinc ion binding were up-regulated $>60$-fold in LT than CO. In addition, PTAC12 (LOC_Os01g56350) involved in a plastid-encoded bacterial-type RNA polymerase (PEP) dependent transcription, pentatricopeptide repeat (PPR) containing proteins (e.g., LOC_Os03g11690 and LOC_Os03g55840), phosphoenolpyruvate/phosphate translocator (OsPPT2; LOC_Os08g25624) involved in dihydroxyacetone phosphate transport and a membrane localized proton-dependent oligopeptide transporter (LOC_Os01g65140) were stronger induced in LT than CO. Furthermore, a putative DNA-binding bifunctional nuclease 1 (OsBBD1; LOC_Os01g50622) involved in abscisic acid-derived callose deposition was 
induced >100-fold in LT than CO. In contrast, splicing factor U2af small subunit A (LOC_Os09g31482) involved in mRNA processing, proliferating cell nuclear antigen (PCNA; LOC_Os02g56130), glucose-6-phosphate 1-dehydrogenase (LOC_Os03g20300) and expressed protein (LOC_Os06g20270) were strongly induced in CO than LT. In addition, terpene synthase (LOC_Os04g27430), and DUF6 containing protein (LOC_Os04g34530) which shares $88 \%$ sequence homology with Walls Are Thin1 (WAT1) were stronger induced in CO than LT.

\section{Expression of peptidyl-prolyl isomerase is elevated in response to $35^{\circ} \mathrm{C}$ plus $M$. oryzae infection}

To investigate the effect of rising temperature and $M$. oryzae infection on both LT and CO, we examined highly induced genes in $\mathrm{CO}+35^{\circ} \mathrm{C}+\mathrm{Mo}$ and $\mathrm{LT}+35^{\circ} \mathrm{C}+\mathrm{Mo}$ treatments. Among the genes with significant up regulation were genes encoding for peptidyl-prolyl isomerase (PPIase, LOC_Os04g28420), HSFA2 (LOC_Os03g53340), photosystem II 10 kDa polypeptide/ chloroplast (LOC_Os07g05360), S-adenosyl-L-methionine benzoic acid/salicylic acid carboxyl methyltransferases and expressed proteins (e.g., LOC_Os09g13440). Perhaps the most compelling observation was the significantly higher expression of peptidyl-prolyl isomerases $(O s F K B P 65)$ in $\mathrm{CO}+35^{\circ} \mathrm{C}+\mathrm{Mo}$ and $\mathrm{LT}+35^{\circ} \mathrm{C}+\mathrm{Mo}$. OsFKBP65 belongs to a class of FK506-binding proteins (FKBPs) with a cis/trans isomerase enzyme activity, some of which can operate as molecular chaperones. OsFKBP65 is a duplicate form of $O S F K B P 64$ both of which were reported as up-regulated by heat treatment (Magiri et al., 2006). OsFKBP65 and OsFKBP64 are orthologous to Arabidopsis AtFKBP65 (ROF2) and AtFKBP62 (ROF1) (Aviezer-Hagai et al., 2007). OsFKBP65 was induced >100fold in $\mathrm{CO}+35^{\circ} \mathrm{C}+\mathrm{Mo}$ and $\mathrm{LT}+35^{\circ} \mathrm{C}+\mathrm{Mo}$, while OsFKBP64 (LOC_Os08g41390) was only slightly up-regulated (7-fold in $\mathrm{CO}$ and 4.5 in LT). The high induction of OsFKBP65 was further confirmed by qRT-PCR in all samples (Figure 2.8).

\section{Differentially expressed resistance $(\mathrm{R})$ gene and $\mathrm{R}$ gene analogues (RGAs)}

Nucleotide-binding and leucine-rich repeat (NB-LRR) R proteins are important for rice resistance response to biotic challenges, including infection with the rice blast fungus (Okuyama et al. 2011). In our dataset, 61 and 75 genes encoding for $\mathrm{R}$ like proteins were upregulated ( $>4$ fold) in $\mathrm{LT}+28^{\circ} \mathrm{C}+\mathrm{Mo}$ and $\mathrm{CO}+28^{\circ} \mathrm{C}+\mathrm{Mo}$, respectively (additional file $\mathrm{S} 4$ ). The number, however, decreased to 50 and 63 in $\mathrm{LT}+35^{\circ} \mathrm{C}+\mathrm{Mo}$ and $\mathrm{CO}+35^{\circ} \mathrm{C}+\mathrm{Mo}$, respectively. 
Database similarity searches indicated that the highly up-regulated $\mathrm{R}$ like genes in $\mathrm{LT}+35^{\circ} \mathrm{C}+\mathrm{Mo}$ and $\mathrm{CO}+35^{\circ} \mathrm{C}+\mathrm{Mo}$ were identical to RPMI (LOC_Os08g16070 and LOC_Os11g11770), PibH8 (LOC_Os08g42700), Xal (LOC_Os04g53160), putative pleiotropic drug resistance proteins (PDR4/PDR8; LOC_Os01g42410 and PDR like ABC transporter; LOC_Os11g37700), RPRI (LOC_Os11g12000) and RPS2 encoding genes (e.g., LOC_Os01g72680 and LOC_Os04g43440). In plants grown at $28^{\circ} \mathrm{C}$, LOC_Os11g45060 was induced 168 and 117-fold in LT and CO, respectively. The gene LOC_Os04g41370, which shares 63\% homology with Pi54 (4.1e-30), was 39 and 17-fold up-regulated in CO and LT, respectively, at $28^{\circ} \mathrm{C}$, but its expression was lower (11-fold in $\mathrm{CO}$ and 8 -fold in LT) at high temperature. We tested the expression of Pi54 (LOC_Os11g42010) using qRT-PCR (Figure 2.9). Pi54 was expressed to a greater extent at $24 \mathrm{hpi}$ than at $48 \mathrm{hpi}$. This probably explains why it was undetected in our samples at $48 \mathrm{hpi}$. Pi54 also exhibited sensitivity to high temperature with reduced expression at $35^{\circ} \mathrm{C}$ in both backgrounds.

\section{Respiratory burst resistance components}

Reactive oxygen species (ROS) play a significant role in plant defense against pathogen attack through the establishment of hypersensitive response (HR). The cell membrane NADPH oxidases (Noxs) are major sources of reactive oxygen species (ROS) under both normal and stress conditions in plants. Oxidative stress gene, OsNox9 (LOC_Os12g35610) was stronger induced in LT compared to $\mathrm{CO}$ at both temperatures, whereas NADPHoxi (RbohD) was stronger induced in $\mathrm{CO}$ than $\mathrm{LT}$, with a stronger induction at $35^{\circ} \mathrm{C}$ compared to $28^{\circ} \mathrm{C}$. To elucidate on this finding, we investigated $\mathrm{H}_{2} \mathrm{O}_{2}$ production in the leaves infiltrated with diaminobenzidine (DAB). Interestingly, intense brown precipitation was observed in all infected plants, and we were unable to convincingly demonstrate any difference in $\mathrm{H}_{2} \mathrm{O}_{2}$ production between plants infected at $28^{\circ} \mathrm{C}$ and $35^{\circ} \mathrm{C}$. We further quantified the accumulation of $\mathrm{H}_{2} \mathrm{O}_{2}$ in plants exposed to $35^{\circ} \mathrm{C}$ and $M$. oryzae infection in comparison with plants exposed to $28^{\circ} \mathrm{C}$. In accordance with callose deposition, both NILs had significantly higher $\mathrm{H}_{2} \mathrm{O}_{2}$ levels during Mo infection of plants pre-exposed to $35^{\circ} \mathrm{C}$ (Figure 2.10A). $\mathrm{H}_{2} \mathrm{O}_{2}$ levels were also consistently higher in $\mathrm{LT}$ than $\mathrm{CO}$ at $28^{\circ} \mathrm{C}$.

Both NADPH oxidases and cell wall peroxidases are known to be mediators of ROS production in response to pathogen attack. More than 20 peroxidase genes were differentially expressed in response to infection compared to $<10$ in response to high temperature 
(additional file S5). Some peroxidase encoding genes were background specific in expression. In the case of LT, LOC_Os04g59200 was >80-fold up-regulated in response to both temperature and infection, while LOC_Os04g59160 was >100-fold up-regulated in CO at $28^{\circ}$ C. LOC_Os04g59190 and LOC_Os01g73200 were expressed in both backgrounds during infection, with a significantly higher up-regulation in CO than in LT. These observations were confirmed by a significant increase in POX activity in CO compared to LT during infection at $28^{\circ} \mathrm{C}, 48$ hpi (Figure 2.10B). However, at 24 hpi, both backgrounds showed increased POX activity in response to Mo. Moreover, POX activity was consistently higher in both backgrounds when subjected to only high temperature, indicating similar temperature sensitivities with respect to POX activity.

\section{Hormone signaling pathway in relation to high temperature induced resistance}

Plant hormones are key signaling molecules in response to stress. Three hormones are of particular significance in plant response to pathogen infection: salicylic acid (SA), jasmonic acid (JA) and ethylene (ET). The phenolic phytohormone, SA, has beneficial effects on plant growth and development under stress conditions (Awasthi et al., 2015). The biosynthesis of SA and other phenolics involved in plant resistance requires the activity of phenylalanine ammonia-lyase (PAL), the first enzyme of the phenylpropanoid pathway (Duan et al., 2014). To determine whether gene expression mirrored SA levels, we compared the levels of free SA and PAL activity in LT and CO plants exposed to $28^{\circ} \mathrm{C}$ and $35^{\circ} \mathrm{C}$ at 24 and 48 hpi. Free SA was consistently higher in $\mathrm{CO}$ than LT in plants grown and inoculated at $28^{\circ} \mathrm{C}$ (Figure 2.11). Surprisingly, free SA significantly increased in both NILs after exposure to $35^{\circ} \mathrm{C}$, although this increase did not last longer in LT. At 48 hpi free SA declined sharply in LT compared to $\mathrm{CO}$; the decline was substantially contrastive at $35^{\circ} \mathrm{C}$ than at $28^{\circ} \mathrm{C}$. There was a comparably similar pattern in PAL activity in LT plants exposed to $35^{\circ} \mathrm{C}$ (Figure 2.12). Although PAL activity was higher in LT than $\mathrm{CO}$ at $24 \mathrm{hpi}$ at both temperatures, there was a sharp decline of PAL activity in LT plants exposed to $35^{\circ} \mathrm{C}$ and Mo at $48 \mathrm{hpi}$, when compared to that of $\mathrm{CO}$ plants.

Genes encoding for SA conversion to MeSA, e.g., S-adenosyl-L-methionine: benzoic acid/salicylic acid carboxyl methyltransferase (SAMT) were highly induced in plants preexposed to $35^{\circ} \mathrm{C}$ than $28^{\circ} \mathrm{C}$ (additional file S6) prior to $M$. oryzae infection. SAMT genes with greater induction included OsBISAMT1 (LOC_Os11g15040), LOC_Os06g13560 and 
LOC_Os11g15300. OsBISAMT1 was also up-regulated >80-fold in response to high temperature in both backgrounds. Analyses of OSBSMT1 expression in wild-type rice plants under various stress conditions was reported to be regulated by jasmonic acid (JA) signaling pathway in rice (Zhao et al. 2010). Although genes encoding for proteins in the jasmonic acid synthesis and signaling pathway were highly induced in both backgrounds, including LOX2 like genes (e.g., LOC_Os08g39840 and LOC_Os08g39850), 12-oxophytodienoate reductases (e.g., LOC_Os06g11200 and LOC_Os06g11240), allene oxide synthase (LOC_Os02g12680) and OsMYB4 (LOC_Os02g41510), there is little evidence supporting their relevance in OsBSAMT1 expression. Previous studies have shown that SA and MeJA conditionally regulate PDF1.2 expression through their activity on NPRl (Mur et al. 2006). However, Jasmonate O-methyltransferases (e.g., LOC_Os06g21820) which catalyze the conversion of JA to MeJA and jasmonate induced jacalin-like lectin (LOC_Os12g14440) that mediates broad spectrum resistance were highly induced in LT than CO. As reported earlier, LT had lower levels of SA compared to CO, suggesting that the positive interaction between MeJA and SA likely occurs at relatively lower levels of SA.

In the ethylene signaling and biosynthetic pathway, we detected $>100$-fold higher induction of a putative constitutive stress-response transcriptional coactivator multiprotein bridging factor 1c (MBF1c; LOC_Os06g39240) at $35^{\circ} \mathrm{C}$ than at $28^{\circ} \mathrm{C}$ in CO, but consistent expression levels were observed in LT at both temperatures. Genes encoding for 1-aminocyclopropane-1carboxylic acid (ACC) synthase (e.g., ACS6; LOC_Os04g48850), a key enzyme in ethylene synthesis, were $>90$ times up-regulated in $\mathrm{CO}$ than LT at both temperatures. In addition, elevated transcript levels of flavonol synthase, a rice ortholog of DMR6 (LOC_Os03g03034) involved in the last step of ethylene synthesis was detected in both backgrounds but with a stronger induction in $\mathrm{CO}$ than LT.

Many genes involved in gibberellins pathway (e.g., GID1L2; LOC_Os03g57640), auxin (e.g., auxin efflux carrier; LOC_Os09g31478) and abscicic acid (e.g., HVA22 (LOC_Os11g05800) were strongly induced in response to Mo, irrespective of the temperature treatment. However, genes encoding for auxin (e.g., OsIAA13) and cytokinin suppressors and ABA responsive genes (e.g., GEM; LOC_Os02g42430) were strongly induced in plants exposed to $35^{\circ} \mathrm{C}$. 


\section{Activated signaling pathways involved in rice defense against pathogen attack}

\section{Receptor like kinases (RLK)}

About 1,100 RLKs that are involved in numerous cellular signaling and developmental events have been reported in rice (Kawano and Shimamoto 2013). Our transcriptome analysis revealed a large number of genes encoding putative receptor kinases (Additional file S7). Within the highly expressed genes, 19 DEGs were annotated as cysteine rich (CRKs), 64 wall associated kinases (OsWAK), 24 SHR5-RLKs, 2 LRRs, 19 S-locus/domain-like receptor protein kinases, 10 senescence-induced receptor-like serine/threonine-protein kinases, 10 Ser/Thr receptor-like kinases and 78 generally annotated as RLKs. The cysteine-rich RLKs (CRKs) represent a prominent subfamily of transmembrane-anchored RLKs, 263 of which are found in rice (Wrzaczek et al., 2010). The CRK encoding genes (e.g., CRK5; LOC_Os04g56430 and LOC_Os04g25060), were >60-fold induced in response to M. oryza infection. S-locus-like receptor protein kinases (e.g., LOC_Os12g03640) were induced >65fold in LT than in CO, whereas lectin-like receptor kinase encoding genes, e.g., LOC_Os07g03780, LOC_Os02g42780 and LOC_Os11g03820, were strongly induced in CO than LT. Genes encoding for wall-associated receptor kinase-like (e.g., OsRLCK146; LOC_Os04g30060) and wall-associated receptor kinase 3 (LOC_Os04g30110) were induced in response to $M$. oryzae and high temperature. OsWAK80, OsWAK60 and OsWAK47 were $>50$-fold up-regulated in both backgrounds, but with a higher induction in CO than in LT. Three SHR5-receptor-like kinase encoding genes, LOC_Os05g17604, LOC_Os05g16430 and LOC_Os05g16920 were up-regulated >50-fold in both backgrounds. Moreover, LOC_Os05g16430 was induced >20-fold in response to only high temperature, suggesting its involvement in heat stress response. Most SHR5 proteins possess leucine-rich repeats (LRR) extracellular domain, placing these receptors into the LRR-RLK family. Interaction network search using PlaNet showed that LOC_Os05g16920 falls in the same interaction network with LOC_Os05g17604 and cytochrome P450 (LOC_Os08g39660), both of which were highly expressed in this study. Cytochromes P450 are mainly involved in alleviating oxidative stress, and activation of allene oxide synthases (AOS). This may suggest that SHR5 proteins perceive oxidative stress signals, and probably act upstream of several signalling molecules, including JA. 


\section{Calcium, calcium-dependent protein kinases}

Cytoplasmic calcium participates in numerous events including signaling, cell membrane permeability and cell wall formation. Calcium metabolism is associated with ROS signaling, and ROS are apparently required to prime $\mathrm{Ca}^{2+}$ influx after elicitation (Torres et al., 2006). Several of $\mathrm{Ca}^{2+}$ binding protein encoding genes, showed reduced expression at high temperature when compared to their expression in response to M. oryzae infection. However, induction of calreticulins (e.g., LOC_Os04g32950) and EF-Hand type family proteins (e.g., LOC_Os03g29770) at $35^{\circ} \mathrm{C}$ than $28^{\circ} \mathrm{C}$ was observed in both backgrounds, and may suggest a possible role in in heat stress response. Plants exposed to double stress involving high temperature and $M$. oryzae infection showed a high induction of calcineurin B (LOC_Os01g41510). Calcineurin B controls responses to stresses, including ion and thermal stresses, to adapt cell growth and proliferation to environmental stresses. With respect to the genetic background, we detected several up-regulated genes involved in calcium signaling during M. oryzae infection (additional file S8). At $28^{\circ} \mathrm{C}$, OsCML21 (LOC_Os05g24780) and OsCML14 were induced $>70$-fold in both backgrounds, with $>20$-fold higher induction in LT than in CO. Putative sodium/calcium exchanger proteins encoding genes (e.g., LOC_Os12g42910 and LOC_Os05g51610) were more up-regulated in LT than in CO. In addition, RelA/SpoT protein (LOC_Os07g42160), which hydrolyses the nucleotide alarmone ppGpp, regulator of cellular metabolism in response to changing environmental conditions was also strongly induced in LT than CO. In contrast, genes encoding for OsCML26, OsCML25 and OsCML2O showed higher induction in CO than in LT. Furthermore, OsCML15, OsCMLA and OsCML31 were stronger induced in CO than in LT at both temperatures.

\section{Mitogen activated protein kinases (MAPKs)}

In rice- $M$. oryzae interaction, MAPKs play a significant role in signaling pathways leading to plant defence. More than 17 MAPKs (OsMPKs) with conserved protein kinase domains I-XI and an activation T-loop of either TEY or TDY motifs have been found in rice (Sheikh et al., 2013). In this study, we detected a significant transcript accumulation of six MAPK encoding genes during infection, including OsMEK5, OsMEK1, OsMPK1, OsMPK5, OsMPK12 and OsMPK13. Interestingly, OsMEK5 (LOC_Os06g09190) was >100-fold up-regulated in $\mathrm{CO}+28^{\circ} \mathrm{C}+\mathrm{Mo}$, but was relatively suppressed in $\mathrm{CO}+35^{\circ} \mathrm{C}+\mathrm{Mo}$. This suppression was probably crucial for effective defense response. OsMPK1 (LOC_Os01g32660) was also up- 
regulated 6-fold in $\mathrm{CO}+28^{\circ} \mathrm{C}+\mathrm{Mo}$ compared to only 4-fold in $\mathrm{LT}+28^{\circ} \mathrm{C}+\mathrm{Mo}$. Protein serine/threonine kinase genes (e.g., OsNPK4; LOC_Os01g50420 and WEE1; LOC_Os02g04240) were induced $>60$-fold in response to infection at both temperatures. OSMAPK5 was up-regulated $>16$-fold in CO during infection at both temperatures but not in LT. OsMPK12 (LOC_Os06g49430) was induced 13-fold in $\mathrm{CO}+35^{\circ} \mathrm{C}+\mathrm{Mo}$ compared to 6fold in $\mathrm{CO}+28^{\circ} \mathrm{C}+\mathrm{Mo}$. Conversely, OsMPK13 (LOC_Os02g04230) was >20-fold induced in $\mathrm{CO}+28^{\circ} \mathrm{C}+\mathrm{Mo}$ compared to 12 -fold in $\mathrm{CO}+35^{\circ} \mathrm{C}+\mathrm{Mo}$. OsMEK1 (LOC_Os01g32660) was 5fold induced in $\mathrm{CO}+35^{\circ} \mathrm{C}+\mathrm{Mo}$ but not in $\mathrm{CO}+28^{\circ} \mathrm{C}+\mathrm{Mo}$. OsMEK1 is lately expressed in response to fungal elicitors and was shown to accumulate in cells inoculated with the avirulent strain of $M$. oryzae (Kim et al., 2000). Expression of these genes was relatively lower in LT at both temperatures. Taken together, differential expression of MAPK encoding genes between LT and CO indicates a significant background influence on their role.

\section{Transcription factors (TFs) induced during high temperature stress}

A total of 2,478 non-redundant set of rice TFs compiled from the Plant Transcription Factor Databases are available in RiceSRTFDB (Priya and Jain 2013). So far, 84 families have been classified based on their domain composition following the rules for each family. TFs exhibited complex expression patterns in response to temperature and M. oryzae in both backgrounds (additional file S9). The majority of TFs were activated in response to M. oryzae compared to high temperature, indicating that the critical transcriptional changes occurred more predominantly in response to pathogen stress than in heat stress. $M Y B$ was the largest group induced in plants subjected to $35^{\circ} \mathrm{C}$, and this was consistent with a high frequency (>90\%) of cis-elements for water stress (MYBCORE) and dehydration stress (MYB1AT) response in both sense and antisense strands of most temperature stress responsive DEGs. Among $M Y B$ genes induced were the Rl-MYB/MYB-like subfamily genes (e.g., LOC_Os01g60850, LOC_Os03g55840 and LOC_Os01g74600), which were common to both backgrounds but were highly induced in LT than CO. Two AP2-EREBP genes with the cis acting element GCC-Box (AGCCGCC); AP2/ERF-138 (LOC_Os09g11480) and LOC_Os05g45954 were induced $>5$ fold in LT but not in CO. Among WRKY genes, OsWRKY111 (LOC_Os05g50700) was strongly induced in both backgrounds. The SWIB/MDM2 complex (LOC_Os03g55310) involved in chromatin remodeling, bZIP genes (e.g., bZIP62; LOC_Os07g48660 and LOC_Os05g16640) and OsMADS25 (LOC_Os04g23910) were >20-fold induced in LT but not in CO. Our dataset also showed 
overlap in the induction of members of homeobox (HB), bHLH, NAC families, and genes that encode zinc-coordinating elements like $\mathrm{C} 2 \mathrm{H} 2, \mathrm{C} 2 \mathrm{H} 2 \mathrm{CO}-\mathrm{like}, \mathrm{C} 3 \mathrm{H}$, GATA, GT (trihelix DNA binding proteins) and histone $\mathrm{H} 2 \mathrm{~A}$ in response to high temperature.

\section{Homeobox (HB), bZIP, AP2-EREBP and MYB transcription factor encoding genes are highly induced during $M$. oryzae infection}

Homeobox is a highly conserved domain in plants, animals and fungi, and their role in maintenance of shoot apical meristem and epidermal development has been demonstrated (Sentoku et al., 2000). In rice, a well-characterized TF in this group is OsBHIDI which encodes a BELL type homeodomain protein (Luo et al., 2005). OsBHIDI was reported to be rapidly induced by $\mathrm{BTH}$ and $M$. oryzae. In our dataset we instead found a high induction of HOX24 and a putative Ser/Thr-rich protein T10 in DGCR region (LOC_Os09g15480) in $\mathrm{LT}+28^{\circ} \mathrm{C}+\mathrm{Mo}$ (additional file S9). HOX24 belongs to the HD-Zip family of HD proteins, characterized by an additional leucine zipper motif adjacent to the HD which facilitates homoand heterodimerization of the transcriptional regulators (Johannesson et al., 2001). HOX24 shares $65 \%$ homology with Arabidopsis $A T H B-6$ which was identified as a negative regulator of ABA signal pathway (Himmelbach et al., 2002). ATHB-6 interacts with abscisic acid insensitive $1(A B 1)$, an upstream phosphatase. In our dataset we instead found higher induction of a putative bZIP transcription factor, ABA INSENSITIVE 5 (ABI5) in LT but not in CO. ABA and OPDA act synergistically to increase levels of $A B I 5$ (Dave et al. 2011). ABI5 is also up-regulated in the ped3 allele, which in turn leads to higher levels of polygalacturonase inhibiting proteins (Kanai et al., 2010). The higher induction of OsBURP16 (LOC_Os10g26940) in LT compared to CO is however intriguing. OsBURP16 belongs to 21 PG1- $\beta$-like sub family of BURP genes and encodes a putative PG1- $\beta$-subunit 22 precursor in rice which was previously reported to be up-regulated by ABA treatment (Ding et al., 2009). PG1s decrease pectin content and cell adhesion and increases abiotic stress sensitivity in rice. The inhibition of PG1 is not well understood. However, internal dynamics leading to inhibition could probably involve $A B I 5$ in the induction of PG1 inhibitors. Another ABA responsive OSBURP gene (OsBURP05; LOC_Os04g14990) from the RD22-like subfamily was induced $>100$ times higher in CO than LT. RD22 is expressed in response to drought stress, and appears to play a different role from OSBURP16. 
We also detected a significant induction of HBP-1b (LOC_Os01g06560) in both backgrounds. In addition, four of the MYB genes, MYB-1, MYB-101, MYB-120 and LOC_Os02g07170, were more induced in LT, while MYB-33 and LOC_Os02g07170 showed $>100$-fold induction in CO. The basic helix-loop-helix encoding gene bHLH25, and auxin response genes OSSAUR2 and OsSOUR15 were strongly induced in CO than in LT. WRKY genes that showed high induction in LT during M. oryzae infection included OsWRKY55, OsWRKY62, OsWRKY28 and OsWRKY71, whereas OsWRKY32, WRKY97, OsWRKY28, OsWRKY27 and OsWRKY84 showed higher induction in CO.

\section{Aspartic proteinases and antimicrobial compounds are highly up-regulated during}

\section{M.oryzae infection.}

Plant aspartic proteinases (APs) have several functions in plants, including protein processing and/or degradation during plant senescence, stress response, programmed cell death, and reproduction. In this study, the putative aspartic proteinase nepenthesin encoding genes (e.g., LOC_Os10g39260 and LOC_Os10g39300) showed >100-fold induction in CO as compared to <100-fold in LT (additional file 10). Both genes are designated as signal peptides in the TIGR database and appear to be homologous. The signal peptides encoded by these genes are involved in hydrolase activity and fall in the super family of acid proteases. The increase in their transcript levels suggests a significant role in rice response to $M$. oryzae. On the other hand, defensins (e.g., DEFL9; LOC_Os06g22919 and DEFL49; LOC_Os04g31250), thionins (e.g., LOC_Os07g24820), lipid transfer proteins LTPs (e.g., LOC_Os10g36070, LOC_Os10g36090 and LOC_Os10g36100), ligatoxin A (e.g., LOC_Os07g46950) and BBTIs were $>20$ fold induced in $\mathrm{LT}+35^{\circ} \mathrm{C}+\mathrm{Mo}$ and $\mathrm{CO}+35^{\circ} \mathrm{C}+\mathrm{Mo}$, suggesting their involvement in rice defense. Genes involved in folate deglutamylation, ubiquitination and subsequent proteasomal degradation of target proteins, including gamma-glutamyl hydrolase (LOC_Os05g44130), SKP1-like protein IB (LOC_Os10g30200) and cullin (CUL1; LOC_Os01g70920) that perceive JA-Ile or giberellin in the nucleus and direct targeted degradation of transcriptional repressors, were stronger induced in CO than LT at high temperature. In contrast, RING-H2 finger proteins encoding for E3 ubiquitin ligase (LOC_Os05g40020), OsFtsH6 FtsH protease (LOC_Os06g12370) involved in oxygenic photosynthesis, peptidase $\mathrm{C} 19$, ubiquitin carboxyl-terminal hydrolase 2 and OsFBO1O involved in signaling were strongly induced in LT than in $\mathrm{CO}$ in response to high temperature. 


\section{Transcriptional regulation of the TCA cycle, carbon and amino acid metabolism}

Under stressful conditions, plant cells must maintain a balance between energy generation and synthesis of structural biomolecules to meet the demands. The glyoxylate shunt in the TCA cycle is of primary importance for the conversion of stored lipids to carbohydrates that serve as an alternative carbon source during stress. Two enzymes: isocitrate lyase and malate synthase are involved in glyoxylate shunt pathway, and contribute to the synthesis of cell components such as cell-wall polysaccharides, nucleotides, and amino acids (Mizuno et al., 2012). In this study, isocitrate lyase (ICL1) and malate synthase encoding genes, LOC_Os07g34520 and LOC_Os04g40990, were >100-fold up-regulated in both backgrounds during M. oryzae infection (Additional file S11). In addition, genes encoding for bifunctional monodehydroascorbate reductase (e.g., LOC_Os11g05520) and dehydrogenase E1 component (e.g., LOC_Os04g02900) were strongly induced in both backgrounds. Genes encoding for citrate synthase, dihydrolipoyl dehydrogenase and aconitate hydratase showed contrasting expression in LT and CO.

Plant defence response requires an abundant supply of energy derived from carbohydrate metabolism. Genes encoding for starch mobilization, including alpha-amylase (Amy3; LOC_Os08g36910), were >60-fold induced in both backgrounds during M. oryzae infection. UDP-glucoronosyl (LOC_Os09g34250) and UDP-glucosyl transferases (LOC_Os02g10880) were induced $>10$-fold in response to high temperature and $M$. oryzae infection in $\mathrm{CO}$ and LT, respectively.

Amino acid metabolism genes encoding for asparagine synthetase, a crucial enzyme in primary nitrogen metabolism, alanine-glyoxylate aminotransferase, isovaleryl-CoA dehydrogenase and 2-aminoethanethiol dioxygenase were $>40$-fold induced in both $\mathrm{CO}$ and LT in response to $M$. oryzae infection, whereas fumarylacetoacetase, 2-isopropylmalate synthase B and D-3-phosphoglycerate dehydrogenase were up-regulated in response to high temperature.

\section{Photosynthesis and lipid metabolism}

Genes involved in photosynthesis and chlorophyll biosynthesis are mostly down-regulated in many plant microbe interaction studies, especially in HR associated plant defence responses (Berger et al., 2007). However, in this study we detected a high induction ( $>50$-fold) of photosystem II $10 \mathrm{kDa}$ polypeptide encoding genes (e.g., LOC_Os07g05360 and 
LOC_Os07g05365) during high temperature stress and M. oryzae infection (Additional file 12). The other genes that were highly induced include glycolate oxidase (GLO3; LOC_Os04g53210), a key enzyme in photorespiration, catalyzing the oxidation of glycolate to glyoxylate. Several cytochrome P450s (e.g., LOC_Os02g36280) were also induced >100fold during $M$. oryzae infection in both backgrounds. Genes encoding for oxygen evolving enhancer protein 3 (OEE3; LOC_Os02g36850) required for photosystem II assembly/stability, chloroplastic group IIA intron splicing facilitator (CRSI; LOC_Os07g30670) involved in chloroplast protein translation and 2Fe-2S iron-sulfur cluster binding protein (LOC_Os07g30670) were >30-fold induced in LT than in CO in response to high temperature. A putative photosystem Q (LOC_Os10g21192) and a photosynthetic reaction center protein (LOC_Os10g39880) were specifically induced in CO at high temperature.

Lipid metabolism is important in the biosynthesis of precursors for plant cuticle synthesis, plant hormones and cell membranes. Interestingly, most genes in the lipid metabolic pathway were either not expressed or down-regulated in plants subjected to $35^{\circ} \mathrm{C}$. Nonetheless, we detected a slight induction of a putative flavonol sulfotransferase (LOC_Os12g17160) in both backgrounds in response to high temperature. One GDSL like gene (LOC_Os01g11730) was highly induced (>100-fold) in both backgrounds, but to a higher level in LT than CO. In contrast, putative lipase encoding genes (e.g., LOC_Os06g10850) were more induced in CO than LT. Genes encoding for omega-6 fatty acid desaturase (LOC_Os07g23410), male sterility protein 2 (fatty acid reductase 1; LOC_Os08g44360), diacylglycerol acyltransferase (LOC_Os09g33530) and thioesterase family protein (LOC_Os09g33530) were highly induced in response to $M$. oryzae infection at both temperatures.

\section{Expression of PR genes}

Pathogenesis related (PR) proteins are rapidly induced by pathogenic infection through the coordinated action of SA, JA and ET signaling pathways. We found a significant overlap in transcript accumulation of several PR encoding genes between $\mathrm{CO}$ and LT. At $35^{\circ} \mathrm{C}$, without $M$. oryzae inoculation, we detected a significant induction of several PR6 genes including RBBI2-0, RBBI2-1, OsOC-6, OsOC-1 and OsWIP1-1, in both backgrounds (Figure 2.13). Conversely, PR3 (CHIT17; LOC_Os05g33130) and PR2 (Gns6; LOC_Os01g71350) were significantly induced in CO but not in LT, whereas PR-5 (TLP-D34; LOC_Os12g43380) was $>10$-fold induced in LT but not in CO. 
In plants inoculated with $M$. oryzae, allergen V5/Tpx-1 related family protein encoding genes were the most highly induced in both CO and LT (>100-fold). Two of these genes, LOC_Os07g03319 and LOC_Os07g03499, were highly induced in LT, while LOC_Os07g0336, LOC_Os07g03279 and LOC_Os07g03458 were highly induced in CO. On the other hand, we detected high induction of PR-1a like genes (e.g., LOC_Os07g03580 and LOC_Os07g03730) in CO more than in LT. High induction (>70-fold) of glucan endo-1,3beta-glucosidases (e.g., Gns12; LOC_Os07g35350, Gns14; LOC_Os07g35350), OsPR4b and OsPR4c was detected in both backgrounds, irrespective of the temperature treatment. Transcript accumulation of the gemine like protein (GLP) encoding genes overlapped between the two backgrounds. GLP1 (LOC_Os04g22080) was >160-fold expressed in LT but was not significantly expressed in CO. In contrast, LOC_Os08g13440 was >200-fold induced in CO compared to only 31-fold in LT. There was, as well, an overlap in the expression of PR-5 encoding genes. PR5 like genes, LOC_Os12g43380, LOC_Os12g43390 and LOC_Os12g43410, were significantly induced in both backgrounds but to a lesser extent in LT than in CO. Conversely, a subtilisin-like serine proteinase, OsSub13, belonging to a class of pathogenesis-related proteins (Jorda and Vera 2000), and a protein-disulfide isomerase (OSPDIL1-1), involved in the biosynthesis of a group of plant defense peptides called cyclotides (Jennings et al., 2001), were more induced in LT than in CO. Expression of glycosyl hydrolase (GH) genes encoding for $\beta-1,3$ glucanases, including LOC_Os01g71830, LOC_Os01g71340 and LOC_Os01g71650, was detected in both CO and LT. Acidic endochitinase (CHIT) encoding genes (e.g., LOC_Os05g15880, LOC_Os11g47500 and LOC_Os06g25010) were the most consistently expressed among the CHIT genes upregulated, and were equally expressed in LT and $\mathrm{CO}$ at both temperatures during pathogen infection. In addition, transcript accumulation of CHIT14, CHIT15 and CHIT16 was also detected in both backgrounds. Our results demonstrated that the expression pattern of PR genes in LT and CO infected samples is somewhat similar, although the level of induction is variable. On the other hand, high temperature affected the transcription of PR genes in both backgrounds, but the magnitude also varied with the genetic background.

\section{Expression of genes involved in secondary metabolism during pathogen infection}

Secondary metabolism, including the production of phytoalexins, plays a significant role in plant defense against pathogens. We analyzed transcripts from both genetic backgrounds for up-regulated DEGs involved in the various metabolic pathways in response to high 
temperature and $M$. oryzae infection. The expression of blast-responsive genes encoding almost all of the biosynthesis enzymes of diterpenoid phytoalexins was either lower or downregulated in plants exposed to only high temperature. However, activated secondary metabolism sub pathways included the phenylpropanoid, flavonoid and terpenoid metabolites (Additional file S13). Among the phenylpropanoids, hydroxycinnamoyl-CoA-shikimate (HCT; LOC_Os11g07960), caffeoyl-3-O-methyltransferase (LOC_Os08g38910) and cinnamyl alcohol dehydrogenase 6 (LOC_Os09g23550) were up-regulated (>4-fold). Phenyalanine ammonia lyase (PAL) encoding genes were down-regulated at high temperature, except PAL2 (LOC_Os02g41670) that showed a >5-fold induction, with significantly higher induction in CO than LT. This is consistent with the higher PAL activity in $\mathrm{CO}$ plants pre-exposed to $35^{\circ} \mathrm{C}$ at 48 hpi reported earlier. During $M$. oryzae infection, genes encoding for O-methyltransferase (OMT), including flavonoid-7-OMT (LOC_Os09g17560) and putative O-methyltransferase ZRP4 (LOC_Os10g02880) were highly induced in LT but not in CO. In contrast, LOC_Os12g13800 was highly up-regulated in $\mathrm{CO}\left(>100\right.$-fold at $35^{\circ} \mathrm{C}, 37$-fold at $\left.28^{\circ} \mathrm{C}\right)$, but not in LT. M. oryzae infection also increased transcript levels of terpene synthase genes (LOC_Os08g07100, LOC_Os04g27190 and LOC_Os04g27670) oryzalexin biosynthesis genes, ent-kaurene synthase (OsKSL8; LOC_Os11g28530 and OsKSL10; LOC_Os12g30824), momilactones biosynthesis genes, entcopalyl diphosphate synthase (OsCPS4; LOC_Os04g09900), (OsKSL4; LOC_Os04g10060) and CYP99A2 (LOC_Os04g10160), and phytocasanes biosynthetic gene, OsKSL7 (LOC_Os02g36140). Additionally, genes encoding for O-hydroxycinnamoyl transferase (LOC_Os04g56910), putative flavanone 3-hydroxylase (LOC_Os10g40934), putative transferase family proteins (LOC_Os08g02030 and LOC_Os04g56910), caffeoyl-CoA-Omethyltransferase (LOC_Os08g38920) putative decarboxylase (LOC_Os08g04540) and chalcone and stilbene synthases (LOC_Os07g34260) were highly induced in both backgrounds during $M$. oryzae infection.

\section{Validation of differential Gene Expression of known temperature and infection responsive rice genes by $\mathrm{qRT}$-PCR}

The expression profiles of known responsive rice genes were validated by checking their up regulation at high temperature and during high temperature pathogen interaction (Figure 2.14). The response of all the measured genes was similar but the magnitude of response was greater in RNA-Seq. This analysis also confirmed the high induction of OsBISAMT1, 
OsFKBP65 (Figure 2.8) and OsWRKY71 in plants pre-exposed to $35^{\circ} \mathrm{C}$ prior to infection compared to $28^{\circ} \mathrm{C}$.

\section{Discussion}

In this study, we used two rice genetic backgrounds carrying Pi54 with a general hypothesis that elevated temperature would weaken rice defenses and cause enhanced pathogen susceptibility, irrespective of the genetic background in which the $\mathrm{R}$ gene is introgressed. The two genotypes showed contrasting reaction to $M$. oryzae at $28^{\circ} \mathrm{C}$, with $\mathrm{CO}$ displaying a more severe disease phenotype than LT. In accordance with restricted lesions sizes observed on infected leaves of LT, intense callose deposits and cell wall florescence were observed in LT than CO, indicating that Pi54 was more effective in $\mathrm{LT}$ than in $\mathrm{CO}$ at $28^{\circ} \mathrm{C}$. This is consistent with the previous reports that Pi54 induces callose deposition (Gupta et al., 2012); although a substantial background influence was detected in this study. Surprisingly, a resistant response was observed in both backgrounds at high temperature. This led us to conclude that resistance in CO background is apparently more temperature dependent than in LT, and that similarities could probably exist between high temperature stress upstream signals and those associated with resistant defense response to $M$. oryzae infection. Indeed, we detected intense cell wall florescence and elevated levels of $\mathrm{H}_{2} \mathrm{O}_{2}$ in both backgrounds at $35^{\circ} \mathrm{C}$, suggesting that induction of ROS and callose may be the first line of defense in plants pre-exposed to high temperature. Intriguingly, HT induced rice defense responses to Mo in both NILs were contrasted by a decrease in Pi54 expression at HT, suggesting that this HT effect enhances plant resistance by either increasing the baseline levels of endogenous Pi54 downstream products thereby decreasing its expression or act independent of Pi54. Thus, it was critical to identify genetic factors that reinforce plant defenses against pathogens at HT. Therefore, an RNA-Seq analysis was carried out to determine whether and how HT and Mo infection alters the transcriptome in the two contrasting NILs.

Our data showed a substantially higher number of DEGs in $M$. oryzae infected rice than in rice subjected to only high temperature, suggesting that rice- $M$. oryzae interaction involved more genome transcriptional regulation than the rice-high temperature interaction. In addition, the response to only high temperature was distinctively different, and clustered separately from both $35^{\circ} \mathrm{C}$-plus- $M$. oryzae and $28^{\circ} \mathrm{C}$-plus- $M$. oryzae treatments. However, combining both stresses sequentially, by exposing plants to high temperature followed by $M$. oryzae 
infection $\left(35^{\circ} \mathrm{C}\right.$-plus- $M$. oryzae), induced both heat and pathogen stress response genes, although disproportionately, suggesting a possible crosstalk between the responses elicited by the two stresses. Our study identified 600 common genes in both high temperature and $M$. oryzae stress conditions. These genes may form a basis for further investigation of crosstalk between abiotic and biotic stress responses. Several genes of unknown function were also induced in plants exposed to high temperature and M. oryzae infection (Figure 2.15), some of them may be a target for further investigations. However, several of the defense response genes showed lower induction in plants subjected to $35^{\circ} \mathrm{C}$-plus-M. oryzae compared to plants inoculated at $28^{\circ} \mathrm{C}$, suggesting that coping with two energetically demanding processes simultaneously prompts the plant to trade off some biochemical processes.

Gene ontology analyses revealed some similarities in the DEGs from both backgrounds during $M$. oryzae infection and in response to high temperature stress. A large part of the modulated transcriptome was devoted to control the main metabolic processes (e.g., oxidation-reduction reactions, photosynthesis, defence response to fungus, carbohydrate, lipid and cellular homeostasis) in both backgrounds. Redox reactions are the basis for many biochemical pathways related to the plants ability to cope with ROS. A stronger enrichment of oxidation-reduction reaction in both genetic backgrounds suggests similarities in oxidative stress response. Heat acclimation was higher enriched in LT compared to CO, suggesting that cellular processes related to heat stress response were more affected in LT compared to CO. In addition, the GO terms pseudouridine synthesis and RNA modification were specific to LT at high temperature (Appendix 2, Figure S1). Pseudouridines ( $\Psi$ ) have been reported to enhance thermodynamic stability of RNA duplexes through substitution of $\Psi$ for $U$ (Kierzek et al. 2014). The significant enrichment of GO:0001522 in LT, suggests a crucial role of pseudouridines in thermo protection of rice cells. The significant enrichment of defense response to fungus, incompatible interaction in $\mathrm{LT}$ compared to $\mathrm{CO}$ in response to $M$. oryzae infection at $28^{\circ} \mathrm{C}$, suggests defense related transcription enhancement in LT than CO, and may be a reason for the differential disease phenotypes between the two genetic backgrounds at $28^{\circ} \mathrm{C}$.

Genes encoding for heat stress response proteins (e.g., OsFKBP65) were among the top upregulated genes in all treatments, and likely perform defense roles in both abiotic and biotic stresses. There are 29 FKBP loci in the rice genome, however, the specific induction of OsFKBP65 suggests its crucial role in both thermotolerance and pathogen defense. Bissoli et 
al. (2012) suggested that ROF2, an orthologue of OsFKBP65, regulates intracellular acid stress, resulting from protein denaturation, by activating $\mathrm{H}^{+}$extrusion. The same authors showed that over-expression of ROF2 activates $K+$ uptake. Indeed we found $>100$-fold induction of a putative potassium transporter encoding gene (LOC_Os03g37930) suggesting a possible involvement of OsFKBP65 in $\mathrm{K}+$ uptake. FKBPs are also implicated in tobacco and wheat disease resistance (Shumilina et al., 2006). A recent study by Narsai et al. (2013) showed overrepresentation of peptidyl isomerases in rice against bacterial and parasitic infection. Consistent with this, a PPI was detected among the four genes induced by SAanalogue in wild-type Arabidopsis, which failed to accumulate in wrky33 mutants (Qiu et al., 2008). We speculate that OSFKBP65 may be a downstream target of SA. OsFKBP65 could also interact with HSFA2 to regulate small HSPs in heat stress response. Previous studies have shown that OsFKBP65 is orthologous to Arabidopsis AtFKBP62 (ROF1), which positively modulates heat tolerance by interacting with AtHSP90.1 and increasing AtHsfA2-mediated accumulation of small HSPs (Meiri and Breiman, 2009; Bissoli et al., 2012). Several HSP encoding genes such as HSP81-1, HSP70s, OsDjC10 and small HSPs were induced in high temperature treated plants, suggesting that the aformentioned studies are consistent with the high induction of $O S F K B P 65$ in this study. Other genes expressed in response to heat stress included several PPR repeat containing proteins, which have been reported to mediate specific RNA processing events including RNA editing, transcript processing, and translation initiation (Kotera et al., 2005). The C2H2-type of zinc finger proteins (e.g., LOC_Os06g34400), which are perceived to constitute a redundant family of transcriptional regulators in plants with 94 so far reported in the $O$. indica rice genome and 113 in $O$. japonica, were also strongly induced in response to high temperature. These proteins regulate target gene expression through DNA binding and have been reported in response to pathogens (Kim et al., 2004). Thus, this C2H2-type of zinc finger proteins may be important in both high temperature and $M$. oryzae defense response.

Several $\mathrm{R}$ genes and pleiotropic drug resistance (PDR)-ABC transporters were stronger induced in plants exposed to $M$. oryzae infection than high temperature in this study. The colocalization of some of these induced RGAs, PDRs and R genes, e.g., PDR4/PDR8 (LOC_Os01g42410) RPS2 (LOC_Os01g72680), Pit (LOC_Os01g05620 and LOC_Os01g05600) on chromosome 1; LOC_Os04g41370, RPS2 (LOC_Os04g43440) and Xa1 (LOC_Os04g53160) on chromosome 4; RGAs (LOC_Os11g45190, LOC_Os11g45180, LOC_Os11g44960, LOC_Os11g45060 and LOC_Os11g45050), PDR like ABC transporter 
(LOC_Os11g37700), RPM1 (LOC_Os11g11770), RPR1 (LOC_Os11g12000) and Pi54 (LOC_Os11g42010) on chromosome 11, suggests that their expression could be in concert with Pi54 defense response. Pleiotropic drug resistance genes are induced by ABA, SA and JA in rice (Moons, 2008), which suggests that PDRs and plant defense response, probably involving $\mathrm{R}$ genes, are somewhat connected. The $\mathrm{ABC}$ transporters have been also reported to be involved in the efflux of an antimicrobial diterpenoid compound, sclareol, on to the leaf surfaces of tobacco, and defense response in Arabidopsis involving inhibition of hypersensitive cell death during pathogen defense (Kobae et al., 2006). It is likely that these ABCs transfer defense proteins, including antimicrobial peptides to the apoplast, and probably regulate cell death in rice- $M$. oryzae interaction. The exact mechanism by which these interactions happen in association with $\mathrm{R}$ genes needs further investigation.

Genes involved in ABA signaling pathway were also significantly induced in this study. For instance, a putative DNA-binding protein encoding gene OsBBDI involved in ABA-derived callose deposition was induced in both backgrounds at high temperature, but with a stronger induction in LT than CO. Consistent with this, a strong induction of OsBURP16 was observed in LT during M. oryzae infection. OsBURP16 belongs to PG1- $\beta$-like subfamily of the BURP family genes induced by $\mathrm{ABA}$, and was recently reported to decrease pectin content in rice leaves (Liu et al., 2013). We speculate that OsBURP16 could probably contribute to damageassociated molecular patterns (DAMPs) commonly referred to as oligogalacturonides (OG), which invoke OG-induced callose deposition through OsBBD1. In fact, a DNA/RNA nuclease has been reported to regulate ABA-dependent stimulation of callose deposition during Botrytis cinerea infection (You et al., 2010). Luna et al. (2010) also showed that callose-promoting growth conditions significantly enhanced levels of basal and PAMPinduced $\mathrm{H}_{2} \mathrm{O}_{2}$, and indicated that callose deposition is preceded by PAMP triggered $\mathrm{H}_{2} \mathrm{O}_{2}$. Hence, the observed variation in callose deposition in LT and CO probably correlates with the timing and levels of $\mathrm{H}_{2} \mathrm{O}_{2}$ accumulation. This is consistent with the stronger induction of OsNox9 in LT than in $\mathrm{CO}$ at $28^{\circ} \mathrm{C}$, but detectable in $\mathrm{CO}$ at $35^{\circ} \mathrm{C}$. On the other hand, $\mathrm{ABA}$ induced callose deposition has been shown to be more suppressed at 5\% compared to $1 \%$ sucrose (Luna et al., 2010). Our data show that genes involved in carbohydrate metabolism leading to sugar accumulation are more induced in CO than LT, suggesting that higher sugar levels could be have a negative impact on callose induction in CO than LT. This is also substantiated by the stronger induction of glucose-6-phosphate 1-dehydrogenase (G6PDH) in CO compared to LT. G6PDH supplies reducing energy to cells by maintaining the level of the 
co-enzyme nicotinamide adenine dinucleotide phosphate (NADPH). The NADPH in turn maintains the level of the antioxidant enzyme glutathione. Besides this, PAMP induced callose was shown to be higher under environmental growth conditions that induced ROS accumulation (reference there in). Hence, the high activity of antioxidants in $\mathrm{CO}$ at $28^{\circ} \mathrm{C}$ likely compromises ROS induced callose deposition. The production of callose is essential for many physiological processes in addition to innate immunity, such as regulation of cell-to-cell movement of molecules, cell plate formation, formation and closure of sieve pores, guard cell wall thickening and establishment of distinct cell cortex domains, which may provide an explanation as to why the rate of callose formation is necessary and faster at high temperatures. In this context, induction of callose by M. oryzae infection in plants treated with high temperature could be associated with increased ROS production and ABA activity. Consistent with this, reduction in sucrose levels as a result of repression of photosynthesis in abiotic stress treated plants further enhances callose deposition. Callose may restrict the fungus to the substomatal space, isolating and possibly starving the fungus. Because ABA induces the transcription of PG1 genes, it is possible that OGs generated from the activity of PG1s invoke intracellular production of JA via the octadecanoid pathway. This is consistent with previous reports (Howe and Schaller, 2008).

Alternatively, it has been shown that ABA binding inhibits the activity of PP2C phosphatases that in turn represses the activity of sucrose non-fermenting 1 (SNF1)-related kinase to relieve repression of key transcription factors (Cutler et al., 2010). Indeed most calcium/calmodulin dependent protein kinases (SNF1-related) were suppressed at high temperature, indicating that ABA played a crucial role in modulating these kinases. On the other hand, LecRLKs have been reported to confer rice resistance to $M$. oryzae, bacterial blight and insect parasites (Singh et al., 2012). The Nicotiana attenuata lectin receptor kinase1 (LecRK1) was identified to be involved in the mechanism used by plants to suppress the Manduca sexta-triggered accumulation of salicylic acid (Bonaventure 2011), suggesting independent signaling from SA. LecRLKs are carbohydrate binding receptors, and this might suggest that OGs are the most likely ligands, and is consistent with a strong induction of a jacalin-like lectin (LOC_Os12g14440) that mediates broad spectrum resistance in LT but not in CO.

Although we detected increased levels of SA in CO at both temperatures, and in LT at high temperature, there is no complete picture of SAR action in rice. Studies show that SA plays a protective role against oxidative stress than defense against pathogens (Iwai et al., 2007). Our 
data shows decreased levels of SA in LT compared to $\mathrm{CO}$ at $28^{\circ} \mathrm{C}$, suggesting that SA may not provide sufficient protection against $M$. oryzae at $28^{\circ} \mathrm{C}$. Indeed SA has been reported to poorly induce PR gene expression and resistance to $M$. oryzae infection (reference therein). Moreover, $\mathrm{R}$ gene mediated resistance in $M$. oryzae infected young rice plants occurs independent of SA (Iwai et al., 2007). Thus, the induction of OsBISAMT1, which converts SA to MeSA, appears to suggest that MeSA could probably participate in a defence response pathway leading to seedling resistance in rice rather than SA. Previous reports have suggested that JA probably antagonizes SA by modulating biosynthesis and activity BSMT1 (Chen et al., 2003). Although MeSA has been reported not to induce a defense response (Seskar et al., 1998), a link between MeSA, lipid derivatives and apoplastic lipid transfer proteins has been reported (Park et al., 2007), indicating that MeSA probably interacts with JA and JA derivatives. Considering that jasmonate-O-methyltransferase, an enzyme that catalyzes the conversion of JA to MeJA was highly induced in LT than CO; it is possible that the lipid derivative that interacts with MeSA is methyl jasmonate (MeJA). MeJA and certain jasmonoyl-amino acid conjugates (e.g., JA-Ile) are potent elicitors of defense gene expression (Howe and Schaller, 2008), and a strong induction of jasmonate-O-methyltransferase in LT but not in $\mathrm{CO}$ suggests the involvement of MeJA in rice defence response.

However, there may be additional signals from HT eventually eliciting defence responses to Mo in CO, which could be explained by other mechanisms. The increased transcript levels of $M B F 1 c$ in $\mathrm{CO}$ plants exposed to $35^{\circ} \mathrm{C}$ compared to $28^{\circ} \mathrm{C}$ may be one other mechanism suggestive of priming towards effective resistance response. Constitutive expression of $M B F 1 c$ in transgenic Arabidopsis plants was previously shown to increase resistance against bacterial infection, heat and osmotic stress by partially activating the ethylene-response signal transduction pathway (Suzuki et al., 2008). Ethylene burst being one of the earliest events in HR (Seo et al., 2011), we speculate that the up-regulation of MBF1c in plants exposed to $35^{\circ} \mathrm{C}$ partially compensates for the reduced resistance at $28^{\circ} \mathrm{C}$ through amplification of the ET signaling pathway. Coupled with this, an increased transcript level of ERF5, an orthologue of Arabidopsis ERF96 was detected in our dataset. ERF96 is up-regulated by ACC, a process that is repressed by SA. The increased transcript levels of this gene, as well as methionine derivatives (e.g., SAMTs) and defensins suggest that ET, in addition to JA, are the major players in the response of HT pre-treated plants to Mo.

Besides this, genes encoding for various kinds of other defence related transcription factors such as U2 small nuclear ribonucleoprotein auxiliary factor $(U 2 A F)$, WRKYs, zinc finger 
RING type, OsEREBPs, MYBs, HB, bHLH, zinc finger (ZF) family and basic region/Leu zipper motif (bZIP) family were induced in response to HT and Mo. $U 2 A F$ plays a crucial role in the recognition of a $3^{\prime}$-splice site which contributes to the fine-tuned control of pre-mRNA splicing. $U 2 A F$ was expressed in all treatments and could be an important candidate to explore the crosstalk between HT and pathogen response. Increased expression of OsWRKY IIa encoding genes was also detected in response to M. oryzae. OsWRKY IIa have been reported to interact or play an indirect role in transcriptional activation of defense-related gene expression by interacting with other regulatory proteins or form combinatorial dimers themselves to regulate target gene expression (Peng et al., 2010). In this study, OsWRKY55 was stronger induced in LT than in $\mathrm{CO}$, indicating that this gene could probably have a positive role in rice defense against $M$. oryzae. This is consistent with the previous reports (Wei et al. 2013). Alternatively, OsWRKY84 was strongly induced in $\mathrm{CO}+35^{\circ} \mathrm{C}+\mathrm{Mo}$ than $\mathrm{CO}+28^{\circ} \mathrm{C}+\mathrm{Mo}$. OsWRKY84 was previously reported to be over-expressed in response to xylanases in suspension-cultured rice cells. $M$. oryzae is known to induce endoxylanases for horizontal expansion in infected leaves (Nguyen et al., 2011). Since the functional role of OsWRKY84 is not well understood, it would be a potential target for further investigation. Induction of OsIAA13, which participates in blocking auxin signaling by repressing auxin response factor (ARF) function as a transcriptional regulator (Kitomi et al., 2012), suggests transcriptional reprogramming towards enhanced heat tolerance, and indirectly pathogen resistance. We also detected increased expression of histone $\mathrm{H} 2 \mathrm{~A}$ encoding genes. $\mathrm{H} 2 \mathrm{As}$ have specialized roles through alterations they create in chromatin stability and folding (Ausio and Abbott, 2002). Their expression reflects a specialized role in plants during high temperature stress, when stability of major cellular functions is required. Interestingly, genes encoding for Histone $\mathrm{H} 2 \mathrm{~A}$ were not expressed during $M$. oryzae infection, suggesting a more significant role in heat stress compared to pathogen stress.

Downstream of TFs could be several genes encoding for defensins, GDSL LIPASE-LIKE 1, thionins, LTPs, PR6, GLPs, and membrane attack complex component/perforin/complement C9 (LOC_Os05g48360), which were stronger induced in $\mathrm{CO}+35^{\circ} \mathrm{C}+\mathrm{Mo}$ than $\mathrm{CO}+28^{\circ} \mathrm{C}+\mathrm{Mo}$. These proteins have fungicidal activity, and could confer resistance through membrane permeabilization of fungal tissues. Several proteinases and proteinase inhibitors induced in this study could also probably play important roles in protein breakdown and recycling, as well as, control of proteolysis by allowing selective mobilization of proteins during stress. However, the stronger induction of these proteases in a relatively susceptible interaction 
$\left(\mathrm{CO}+28^{\circ} \mathrm{C}+\mathrm{Mo}\right)$ compared to a resistant response $\left(\mathrm{LT}+28^{\circ} \mathrm{C}+\mathrm{Mo}, \mathrm{CO}+35^{\circ} \mathrm{C}+\mathrm{Mo}\right.$ $\mathrm{LT}+35^{\circ} \mathrm{C}+\mathrm{Mo}$ ) is intriguing, and could probably suggest that these proteinases play a role in M. oryzae successful colonization of the host.

Genes involved in primary metabolism, secondary metabolism and PRs were more induced in response to $M$. oryzae than high temperature. Genes involved in photosynthesis were also suppressed at high temperature. However, genes encoding for photosystem II $10 \mathrm{kDa}$ polypeptides were induced at all conditions, suggesting that these proteins probably play a significant role in protecting rice PSII from oxidative perturbations induced by heat stress and pathogen infection.

Overall, the present biochemical and transcriptome analysis provided a broad insight into biological processes potentially involved in rice response to HT and Mo, including representatives of all major classes of defence genes. We have shown that rice lines carrying Pi54 respond to Mo by rapid induction of callose and $\mathrm{H}_{2} \mathrm{O}_{2}$, and that these resistance mechanisms are amplified at HT. Coupled with this, our analyses show that MeSA, MeJA and ET have a role in enhancing defence response to Mo after HT treatment. Crosstalk among these pathways appears to be a key defence mechanism against Mo. Other than these molecules, increased expression of genes in the Auxin, ABA and gibberellin signaling pathways was also detected. However, increased expression of negative regulators of these pathways such as OsIAA13, OsGH3.2, OsGH3.5, ARF19, ERF5, and OsBBD1 involved in ABA-derived callose deposition, suggests that internal dynamics are involved in fine-tuning the negative defence responses associated with this hormones. For instance, ARF19 is induced by either IAA or ethylene treatments, but could be inactivated by the binding of OSIAA13 to decrease its sensitivity to auxin. GH3 genes encode IAA-conjugating enzymes, which could also act as feedback regulators by reducing free auxin levels. Thus, multiple regulatory pathways coupled with genetic background factors are involved in achieving potentially effective resistance in plants exposed to elevated temperatures. We have not linked our transcriptome analysis with functional analysis. It is possible that temperature might significantly modify post-transcriptional regulation. Thus, the effects of temperature on plant defense related genes identified in this study may require further investigation at the post transcriptional level. Equally, we do not know how much callose is required to completely restrict the pathogen, and whether temperatures of $35^{\circ} \mathrm{C}$ would produce the same results in plants without $\mathrm{R}$ gene mediated resistance. Nonetheless, the interaction between high 
temperature and $M$. oryzae infection widens our understanding of how more than one stress factor and the genetic background influence rice defense responses.

Materials and Methods

\section{Plant and fungal material and rice blast inoculation}

Two genotypes, Li-Jiang-Xin-Tuan-He-Gu (LT, Oryza japonica) and Co39 (Oryza indica) carrying the Pi54 R gene, were used as hosts in this experiment. LT is widely identified as a genetic background for the development of NILs, whereas Co39, a short duration $O$. indica cultivar from India with high susceptibility to tropical blast isolates, was recently introduced as a second background for the development of NILs. The plants were grown in the greenhouse at $27 \pm 2^{\circ} \mathrm{C}$ with a photoperiod of $14 \mathrm{~h}$ for 21 days and then transferred to growth chambers at $28^{\circ} \mathrm{C}$ and $35^{\circ} \mathrm{C}$ for 7 days. After high temperature exposure, all plants were kept at $28^{\circ} \mathrm{C}$ for inoculation to allow fungal infection at equal conditions. The fungal strains were cultured on V8 agar plates at $25^{\circ} \mathrm{C}$. Inoculation was performed using a blast isolate, TAN211.16, from Tanzania. The inoculated plants were incubated for $24 \mathrm{~h}$ at $28^{\circ} \mathrm{C}$ and $90 \%$ relative humidity in the dark and then transferred back to $12 \mathrm{~h}$ of light for additional $24 \mathrm{~h}$ before leaf sampling. Some plants were maintained at $35^{\circ} \mathrm{C}$ and $28^{\circ} \mathrm{C}$ after inoculation for disease assessment. Leaf blast was assessed from 4 to 12 days after inoculation. Disease assessment was repeated two times to confirm the presence of lesions. The area under the disease progress curve (AUDPC) was calculated using the trapezoidal method.

\section{Illumina HiSeq2000 Sequencing}

The RNAseq gene expression analysis experiment was designed as a three-factor experiment with eight treatments: (1) $\mathrm{LT}+35^{\circ} \mathrm{C}+\mathrm{Mo}$; (2) $\mathrm{LT}+28^{\circ} \mathrm{C}+\mathrm{Mo}$; (3) $\mathrm{LT}+35^{\circ} \mathrm{C}$; (4) $\mathrm{LT}+28^{\circ} \mathrm{C}$; (5) $\mathrm{CO}+35^{\circ} \mathrm{C}+\mathrm{Mo}$; (6) $\mathrm{CO}+28^{\circ} \mathrm{C}+\mathrm{Mo}$; (7) $\mathrm{CO}+35^{\circ} \mathrm{C}$; (8) $\mathrm{CO}+28^{\circ} \mathrm{C}$. These treatments represent the genetic background ( $\mathrm{LT}$ or $\mathrm{CO})$, temperature $\left(28^{\circ} \mathrm{C}\right.$ and $\left.35^{\circ} \mathrm{C}\right)$ and $M$. oryzae inoculation (Mo). The experiment included three biological replicates. Each sample represented leaf material from five plants. Total RNA was isolated using Trizol reagent (Invitrogen, Carlsbad, CA) according to the manufacturer's instructions. The integrity of each RNA sample obtained was examined by Agilent Lab-on-a-chip technology using the RNA 6000 Nano LabChip kit and a Bioanalyzer 2100 (Agilent Technologies, Palo Alto, CA). Two micrograms of total RNA were subjected to library preparation using the 'TruSeq RNA Sample preparation v2 Guide' following the manufacturer's instructions. Briefly, the poly-A containing mRNAs 
were purified by using poly-T oligo-attached magnetic beads and then fragmented. The first and second cDNA strands were synthesized, end repaired, and adaptors were ligated after adenylation at the 3'-ends. cDNA fragments containing adaptors on both ends were selectively enriched by 15 cycles of PCR amplification. The indexed Illumina libraries were validated for fragment size using the Agilent High-Sensitivity cDNA chip on 2100 Bioanalyzer. Libraries were clustered for sequencing using Illumina cBOT Cluster generation system and TrusSeq v3 chemistry. Fifty bp single-end sequencing was performed on the HiSeq2000 using three barcoded libraries per lane.

\section{Preprocessing, Mapping, and analysis of illumina reads and subsequent gene expression data}

Sequence images were transformed with Illumina software BaseCaller to bcl files, which were demultiplexed to fastq files with CASAVA v1.8.2 allowing for one mismatch in the indices. Sequences were aligned by Bowtie2 v2.0.2 to the Ensembl cDNA release 16 for Oryza sativa. Subsequently, conversion and sorting of the resulting sam files to sorted bam files was conducted with samtools 0.1 .18 followed by counting the reads to each gene. Data was preprocessed and analysed in the R/Bioconductor environment loading DESeq, lattice, estrogen, gplots and colorRamps, biomaRt packages. After filtering the genes exceeding more than 50 counts for at least one sample, normalization (via Trimmed Mean and Medianvalues), estimation of dispersions and testing for differentially expressed genes based on a $\chi^{2}$ likelihood ratio test, assuming negative binomial data distribution were computed via DESeq. Candidate genes were filtered to a minimum of $4 \mathrm{x}$ fold change and FDR-corrected $\mathrm{P}$ value $<0.05$. Gene annotation was conducted using Ensembl Biomart via R. Functional association enrichment analysis was conducted via the R package goseq. RPKM values were calculated via CLC Genomics Workbench software (CLC Bio, Swansea, UK). We generated hierarchical clustering heatmaps using Multi experiment Viewer (MEV TM4 Microarray Software Suite, v4.9; Dana-Farber Cancer Institute) using average linkage Pearson correlation hierarchical clustering. The identification of cis elements was performed using Osiris software (available at http://www.bioinformatics2.wsu.edu/cgi-bin/Osiris/cgi/home.pl), which searches known cis elements from PLACE - Plant Cis-acting Regulatory DNA Elements (Higo et al., 1999) database. In order to verify if the elements were not randomly presented, a $Z$ test was performed. The probability (p) for each motif was obtained and the elements with p-values equal to or less than 0.05 were considered as non-random. Cis elements with higher occurrence among up-regulated DEGs were identified. 


\section{Histochemistry and microscopy}

Callose staining was carried out according to the method of Hao et al. (2008), with minor modifications. Briefly, leaf sections were destained by boiling in methanol:acetic acid (3:1) at $72^{\circ} \mathrm{C}$ and rinsed first in $50 \%$ ethanol and then in water. The destained leaves were stained for $1 \mathrm{~h}$ at room temperature in a solution containing $0.1 \%(\mathrm{w} / \mathrm{v})$ aniline blue in $0.15 \mathrm{M} \mathrm{K}_{2} \mathrm{HPO}_{4}$ and examined under the UV epifluorescence microscope. Diaminobenzidine (DAB) staining of hydrogen peroxide was carried out according to Thordal-Christensen et al. 1997 with some modifications. Briefly, destained leaf sections were immersed in $1 \mathrm{mg} \mathrm{ml}^{-1} \mathrm{DAB}$ solution and incubated in the dark overnight. The leaf sections were rinsed in double distilled water before microscopic examination.

\section{Measurement of hydrogen peroxide $\left(\mathrm{H}_{2} \mathrm{O}_{2}\right)$}

Leaf samples were collected 24 and 48 hai and immediately ground in liquid nitrogen. The ground powder was extracted in $5 \% \mathrm{w} / \mathrm{v}$ metaphosphoric acid. The samples were centrifuged at $5000 \times \mathrm{g}$ for $60 \mathrm{~min}$. The supernatant was adjusted to $\mathrm{pH} 6.5$ using $1 \mathrm{M}$ tricine in $6 \mathrm{M}$ $\mathrm{NaOH}$, and the initial amount of NADH was measured at $340 \mathrm{~nm}\left(\mathrm{OD}_{1}\right)$ using SPECORD 40 (Analytik, Jena, Germany). Horseradish peroxidase (350 $\mu \mathrm{l}^{-1} ; 2500 \mathrm{U} \mathrm{ml}^{-1}$ ) dissolved in $25 \mathrm{mM}$ sodium phosphate buffer ( $\mathrm{pH}$ 7.0) was added and the mixture was incubated at room temperature. The absorbance changes at $340 \mathrm{~nm}$ were measured at $25^{\circ} \mathrm{C}, 60 \mathrm{~min}$ after the addition of horseradish peroxidase $\left(\mathrm{OD}_{2}\right)$. The $\mathrm{H}_{2} \mathrm{O}_{2}$ contents were determined by a calibration curve using known amounts of $\mathrm{H}_{2} \mathrm{O}_{2}$ and expressed as micromoles per gram of fresh weight $(\mathrm{FW})$.

\section{Guaiacol peroxidase and PAL activity}

Fresh leaf samples weighing $300 \mathrm{mg}$ were homogenized in $3 \mathrm{ml}$ of cold $100 \mathrm{mM} \mathrm{Na}$ phosphate buffer ( $\mathrm{pH}$ 7.0). The homogenates were centrifuged at $5000 \mathrm{x} \mathrm{g}$ for $30 \mathrm{~min}$, and the dialyzed enzyme extracts were used for the assay. Assay mixture in a total volume of $3 \mathrm{ml}$ contained $100 \mathrm{mM}$ Na-phosphate buffer (pH 7.0), 50 $\mu 1$ of $20 \mathrm{mM}$ guaiacol solution, $30 \mu \mathrm{l}$ of $12.3 \mathrm{mM}$ hydrogen peroxide and $50 \mu \mathrm{l}$ of enzyme extract. Increase in absorbance was measured at $420 \mathrm{~nm}$ (extinction coefficient of $26.6 \mathrm{mM} \_1 \mathrm{~cm} \_1$ ) at $30 \mathrm{~s}$ intervals up to $3 \mathrm{~min}$, using SPECORD 40, Analytikjena AG (Germany). Enzyme specific activity was expressed as mKatal. PAL activity was determined according to Ueno et al. (2011). 


\section{Salicylic acid determination}

Accumulation of salicylic acid (SA) was examined through high performance liquid chromatography (HPLC) analysis. SA extraction was carried out according to a modified method of Kamble and Bhargava (2007) with minor modifications. Briefly, 300mg of ground leaf tissue was homogenized in $3 \mathrm{ml}$ acetone and centrifuged at $3800 \mathrm{x} \mathrm{g}$ for $60 \mathrm{~min}$ in a refrigerated centrifuge (Sigma 4K10). For determination of free SA, the supernatant was transferred to a new tube and acetone was evaporated at $35^{\circ} \mathrm{C}$ for about 2 hours. The dry residue was dissolved in HPLC grade water and partitioned against an equal volume of ethyl acetate. The ethyl acetate layer was evaporated to dryness at $35^{\circ} \mathrm{C}$, and the residue was finally dissolved in $500 \mu 1$ of HPLC grade methanol. SA samples were subjected to HPLC separation using a C18 (LiChrospher 100 Merck) reverse phase column $(25 \mathrm{cmx} 4 \mathrm{~mm}$ id), at a flow rate of $1 \mathrm{ml} \mathrm{min}^{-1}$ in a gradient program for the solvents: (A) $20 \mathrm{mM} \mathrm{NaAc}$ and (B) methanol. SA was detected using a fluorescence detector with excitation and emission wavelengths of 315 and $405 \mathrm{~nm}$, respectively.

\section{Real-time RT-PCR analysis}

Total RNA was isolated using Trizol reagent (Invitrogen, Gaithersburg, MD, USA) according to the manufacturer's instructions. Relative gene expression was determined by quantitative real time PCR (RT-PCR) on a Bio-Rad CFX384 real time system using SYBR Green dye (Bio-Rad, California, USA). The primer efficiency was determined and taken into account when evaluating the qRT-PCR data. The data were normalized to the geometrical mean of a reference gene (LOC_Os07g02340), previously found to show a stable expression pattern across biotic and abiotic stress (Narsai et al. 2010). The Pfaffl method was used to analyse the qRT-PCR data in Microsoft Excel, and the calculated values were expressed relative to the control. Primer sequences are listed in (Additional file S14).

\section{Acknowledgement}

This work was supported by BMZ funded project "Mitigating the impact of climate change on rice diseases in East Africa". We thank the Microarray and Deep-Sequencing Core Facility of the Developmental Biochemistry University Medical Center Göttingen (UMG) for performing the RNA-seq analysis. In particular, we would like thank Fabian Ludewig for successfully 
running illumina sequencing. Our sincere thanks are expressed to Dagmar Tacke, Marian Süß and Daniel Kretzschmar for their technical support.

\section{References}

Ausio, J., and Abbott, D. W. 2002. The many tales of a tail: carboxyl-terminal tail heterogeneity specializes histone $\mathrm{H} 2 \mathrm{~A}$ variants for defined chromatin function. Biochemistry 41:5945-5949.

Aviezer-Hagai, K., Skovorodnikova, J., Galigniana, M., Farchi-Pisanty, O., Maayan, E., Bocovza, S., Efrat, Y., von Koskull-Doring, P., Ohad, N. and Breiman, A. 2007. Arabidopsis immunophilins ROF1 (AtFKBP62) and ROF2 (AtFKBP65) exhibit tissue specificity, are heat-stress induced, and bind HSP90. Plant Mol. Biol. 63:237-255.

Berger S, Benediktyová Z., Matous K., Bonfig K., Mueller M. J., Nedbal L., Roitsch T. 2007. Visualization of dynamics of plant-pathogen interaction by novel combination of chlorophyll fluorescence imaging and statistical analysis: differential effects of virulent and avirulent strains of $P$. syringae and of oxylipins on A. thaliana. J Exp Bot. 58:797806.

Bieri, S., Mauch, S., Shen, Q. H., Peart, J., Devoto, A., Casais, C., Ceron, F., Schulze, S., Steinbiss, H. H., Shirasu, K., et al. 2004. RAR1 positively controls steady state levels of barley MLA resistance proteins and enables sufficient MLA6 accumulation for effective resistance. Plant Cell 16:3480-3495.

Bissoli, G., Niñoles, R., Fresquet, S., Palombieri, S., Bueso, E., Rubio, L., GarcíaSánchez, M. J., Fernández, J. A., Mulet, J. M., and Serrano, R. 2012. Peptidyl-prolyl cis-trans isomerase ROF2 modulates intracellular $\mathrm{pH}$ homeostasis in Arabidopsis. Plant J. 70:704-716.

Bonaventure, G. 2011. The Nicotiana attenuata Lectin Receptor Kinase 1 is involved in the perception of insect feeding. Plant Signal Behav. 6:1-4.

Chen, F., D'Auria, J. C., Tholl, D., Ross, J. R., Gershenzon, J., Noel, J. P., and Pichersky, E. 2003. An Arabidopsis thaliana gene for methylsalicylate biosynthesis, identified by a biochemical genomics approach, has a role in defense. Plant J. 36:577-588.

Cheong, Y. H., Moon, B. C., Kim, J. K., Kim, C. Y., Kim, M. C., Kim, I. H., Park, C. Y., Kim, J. C., Park, B. O., Koo, S. C., Yoon, H. W., Chung, W. S. et al. 2003. BWMK1, a rice mitogen-activated protein kinase, locates in the nucleus and mediates pathogenesisrelated gene expression by activation of a transcription factor. Plant Physiology 132:19611972.

Cutler, S. R., Rodriguez, P. L., Finkelstein, R. R., and Abrams, S. R. 2010. Abscisic acid: emergence of a core signaling network. Annu. Rev. Plant Biol. 61:651-679. 
Dave, A., Hernández, M. L., He, Z., Andriotis, V. M. E., Vaistij. F. E., Larson, T. R., and Graham, I. A. 2011. 12-Oxo-phytodienoic acid accumulation during seed development represses seed germination in Arabidopsis. Plant Cell. 23:583-599.

Ding, D., Zhang, L., Wang, H., Liu, Z., Zhang, Z. and Zheng, Y. 2009. Differential expression of miRNAs in response to salt stress in maize roots. Ann. Bot. 103:29-38.

Eitzinger, J., Orlandini, S., Stefanski, R., and Naylor, R. E. L. 2010. Climate change and agriculture: introductory editorial. J. Agri. Sci. Cambridge 148:499-500.

Elmore, J.M., Lin, Z-J.D., and Coaker G. 2011. Plant NB-LRR signaling: upstreams and downstreams. Current Opinion in Plant Biology 14:365-371.

Fu, D. L., Uauy, C., Distelfeld, A., Blechl, A., Epstein, L., Chen, X., Sela, H., Fahima, T., and Dubcovsky, J. 2009. A Kinase-START Gene confers temperature-dependent resistance to wheat stripe rust. Science 323:1357-1360.

Ghini, R., Hamada, E., and Bettiol, W. 2008. Climate change and plant diseases. Sci. Agric. (Piracicaba, Braz.) 65:98-107.

Gupta, S. K., Rai, A. K., Kanwar, S. S., Chand, D., Singh, N. K., et al. 2012. The single functional blast resistance gene Pi54 activates a complex defense mechanism in rice. $\mathrm{J}$ Exp. Bot. 63:757-772.

Himmelbach, A., Hoffmann, T., Leube, M., Hohener, B., Grill, E. 2002. Homeodomain protein ATHB6 is a target of the protein phosphatase ABI1 and regulates hormone responses in Arabidopsis. EMBO J. 21:3029-3038.

Howe, G. A., and Schaller A. 2008. Direct defense in plants and their induction by wounding and insect herbivores. In: Schaller A. (Editor) Induced plant resistance to herbivory, Springer Science + Business Media B.V. 7-29.

Iwai, T., Seo, S., Mitsuhara, I., and Ohashi, Y. 2007. Probenazoleinduced accumulation of salicylic acid confers resistance to Magnaporthe grisea in adult rice plants. Plant Cell Physiol. 48:915-924.

Jennings, C., West, J., Waine, C., Craik, D., and Anderson, M. 2001. Biosynthesis and insecticidal properties of plant cyclotides: The cyclic knotted proteins from Oldenlandia affinis. Proc. Natl. Acad. Sci. USA. 98:10614-10619.

Johannesson, H., Wang, Y., and Engstrom, P. 2001. DNA-binding and dimerization preferences of Arabidopsis homeodomain-leucine zipper transcription factors in vitro. Plant Mol. Biol. 45:63-73.

Jorda, L., and Vera, P. 2000. Local and systemic induction of two defense-related subtilisinlike protease promoters in transgenic Arabidopsis plants. Luciferin induction of PR gene expression. Plant Physiol. 124:1049-1057. 
Jorgensen, T. H. 2012. The effect of environmental heterogeneity on RPW8-mediated resistance to powdery mildews in Arabidopsis thaliana. Ann. Bot. 109:833-842.

Jung, K. H., Gho, H. J., Giong, H. K., Chandran, A. K., Nguyen, Q. N., Choi, H., Zhang, T., Wang, W., Kim, J. H., Choi, H. K., and An, G. 2013. Genome-wide identification and analysis of Japonica and Indica cultivar-preferred transcripts in rice using 983 Affymetrix array data. Rice 6:19.

Kanai, M., Nishimura, M., and Hayashi, M. 2010. A peroxisomal ABC transporter promotes seed germination by inducing pectin degradation under the control of ABI5. Plant J. 62:936-947.

Kawano, Y., and Shimamoto, K. 2013. Early signaling network in rice PRR-mediated and R-mediated immunity. Curr. Opin. Plant Biol. 16:496-504.

Kierzek, E., Malgowska, M., Lisowiec, J., Turner, D. H., Gdaniec, Z. and Kierzek, R. 2014. The contribution of pseudouridine to stabilities and structure of RNAs. Nucl. Acids Res. 42:3492-3501.

Kim, C. Y., Lee, S. H., Park, H. C., Bae, C. G., Cheong, Y. H., Choi, Y. J., Han, C., Lee, S. Y., Lim, C. O. and Cho. M. J. 2000. Identification of rice blast fungal elicitorresponsive genes by differential display analysis. Mol. Plant Microbe. Interact. 13:470474.

Kim, H. S., and Delaney, T. P. 2002. Over-expression of TGA5, which encodes a bZIP transcription factor that interacts with NIM1/NPR1, confers SAR-independent resistance in Arabidopsis thaliana to Peronospora parasitica. Plant J. 32:151-163.

Kim, S. H., Hong, J. K., Lee, S. C., Sohn, K. H., Jung, H. W., and Hwang, B. K. 2004. CAZFP1, Cys2/His2-type zinc-finger transcription factor gene functions as a pathogeninduced early-defense gene in Capsicum annuum. Plant Mol. Biol. 55:883-904.

Kim, S. T., Cho, K. S., Yu, S., Kim, S. G., Hong, J. C., Han, C. D., Bae, D. W., Nam, M. H., and Kang, K. Y. 2003. Proteomic analysis of differentially expressed protein induced by rice blast fungus and elicitor in suspension-cultured rice cells. Proteomics 3:2368-2378.

Kitomi, Y., Inahashi, H., Takehisa, H., Sato, Y., and Inukai Y. 2011. OsIAA13-mediated auxin signaling is involved in lateral root initiation in rice. Plant Sci. Int. J. Exp. Plant Biol. 201190:116-122.

Kobae, Y., Sekino, T., Yoshioka, H., Nakagawa, T., Martinoia, E., and Maeshima M. 2006. Loss of AtPDR8, a plasma membrane ABC transporter of Arabidopsis thaliana, causes hypersensitive cell death upon pathogen infection. Plant Cell Physiol. 47: 309-318

Koo, Y. J., Kim, M. A., Kim, E. H., Song, J. T., Jung, C., Moon, J. K., Kim, J. H., Seo, H. S., Song, S. I., Kim, J. K., Lee, J. S., Cheong, J. J., and Choi, Y. D. 2007. Overexpression of salicylic acid carboxyl methyltransferase reduces salicylic acidmediated pathogen resistance in Arabidopsis thaliana. Plant Mol. Biol. 64:1-15. 
Kotera, E., Tasaka, M., and Shikanai, T. 2005. A pentatricopeptide repeat protein is essential for RNA editing in chloroplasts. Nature 433:326-30.

Liu, H., Ma, Y., Chen, N., Guo, S., Liu, H., Guo, X., Chong, K., and Xu, X. 2013. Overexpression of stress-inducible OsBURP16, the $\beta$-subunit of polygalacturonase 1 , decreases pectin content and cell adhesion and increases abiotic stress sensitivity in rice. Plant Cell and Environ. 37:114-1158

Luna, E., Pastor, V., Robert, J., Flors, V., Mauch-Mani, B., and Ton, J. 2011. Callose deposition: a multifaceted plant defense response. Mol Plant Microbe Interact. 24:183-193.

Luo, H., Song, F., and Zheng, Z. 2005. Overexpression in transgenic tobacco reveals different roles for the rice homeodomain gene OsBIHD1 in biotic and abiotic stress responses. J.Exp. Bot. 56:2673-2682.

Magiri, E N., Farchi-Pistany, O., Avni, A., and Breiman, A. 2006. The expression of the large rice FK506 binding proteins demonstrate tissue specificity and heat stress responsiveness. Plant Sci. 170:695-704.

Mizuno, H., Kawahigashi, H., Kawahara, Y., Kanamori, H., Ogata, J., Minami, H., Itoh, T., and Matsumoto, T. 2012. Global transcriptome analysis reveals distinct expression among duplicated genes during sorghum-interaction. BMC Plant Biol. 29:12:121.

Moons, A. 2008. Transcriptional profiling of the PDR gene family in rice roots in response to plant growth regulators, redox perturbations and weak organic acid stresses. Planta 229:5371.

Mur LA, Kenton P, Atzorn R, Miersch O, Wasternack C. 2006. The outcomes of concentration-specific interactions between salicylate and jasmonate signaling include synergy,antagonism, and oxidative stress leading to cell death. Plant Physiology. 140:249262.

Narsai, R., Wang, C., Chen, J., Wu, J., Shou, H., and Whelan, J. 2013. Antagonistic, overlapping and distinct responses to biotic stress in rice (Oryza sativa) and interactions with abiotic stress. BMC Genomics. 14:93.

Negeri, A., Wang, G-F., Benavente, L., Kibiti, C. M., Chaikam, V., Johal, G., and BalintKurti, P. 2013. Characterization of temperature and light effects on the defense response phenotypes associated with the maize Rpl-D21 autoactive resistance gene. BMC Plant Biol. 13:106.

Niinemets, U. 2010. Responses of forest trees to single and multiple environmental stresses from seedlings to mature plants: past stress history, stress interactions, tolerance and acclimation. Forest Ecology and Management 260:1623-1639.

Noutoshi, Y., Ito, T., Seki, M., et al. 2005. A single amino acid insertion in the WRKY domain of the Arabidopsis TIR-NBS-LRR-WRKY-type disease resistance protein SLH1 
(sensitive to low humidity 1) causes activation of defense responses and hypersensitive cell death. Plant J. 43:873-888.

Okuyama, Y., Kanzaki, H., Abe, A., Yoshida, K., Tamiru, M., Saitoh, H., Fujibe, T., Matsumura, H., Shenton, M., Galam, D.C., Undan, J., Ito, A., Sone, T., and Terauchi, R. 2011. A multifaceted genomics approach allows the isolation of the rice Pia-blast resistance gene consisting of two adjacent NBS-LRR protein genes. Plant J. 66:467-479.

Park, H. L., Lee, S. W., Jung, K. H., Hahn, T. R., and Cho, M. H. 2013. Transcriptomic analysis of UV-treated rice leaves reveals UV-induced phytoalexin biosynthetic pathways and their regulatory networks in rice. Phytochemistry. 96:57-71

Park, S.W., Kaimoyo, E., Kumar, D., Mosher, S., and Klessig, D.F. 2007. Methyl salicylate is a critical mobile signal for plant systemic acquired resistance. Science 318:113-116.

Priya, P., and Jain, M. 2013. RiceSRTFDB: A database of rice transcription factors containing comprehensive expression, cis-regulatory element and mutant information to facilitate gene function analysis. Database 2013: article ID bat027.

Qiu J. L., Fiil, B. K., Petersen, K., Nielsen, H. B., Botanga, C. J., Thorgrimsen, S., et al. 2008. Arabidopsis MAP kinase 4 regulates gene expression through transcription factor release in the nucleus. EMBO J. 27:2214-2221.

Rabbani, M. A., Maruyama, K., Abe, H., Khan, M. A., Katsura, K., Ito, Y., Yoshiwara, K., Seki, M., Shinozaki, K., and Yamaguchi-Shinozaki, K. 2003. Monitoring expression profiles of rice genes under cold, drought, and high-salinity stresses and abscisic acid application using cDNA microarray and RNA get-blot analyses. Plant Physiol. 133:17551767

Ren, D., Yang, H., and Zhang S. 2002. Cell death mediated by MAPK is associated with hydrogen peroxide production in Arabidopsis. J. Biol. Chemistry 277:559-565.

Reyna, N. S., and Yang, Y. 2006. Molecular analysis of the rice MAP kinase gene family in relation to Magnaporthe grisea infection. Mol. Plant-Microbe. Interact. 19:530-540.

Rojas, C. M., and Mysore, K. S. 2012. Glycolate oxidase is an alternative source of H2O2 production during plant defense responses and functions independently from NADPH oxidase. Plant Signaling \& Behavior 7:752-755

Sentoku, N., Sato, Y., and Matsuoka, M. 2000. Overexpression of rice OSH genes induces ectopic shoots on leaf sheaths of transgenic rice plants. Dev. Bio. 220:358-364.

Seo, S., Mitsuhara, I., Feng, J., Iwai, T., Hasegawa, M., and Ohashi, Y. 2011. Cyanide, a co-product of plant hormone ethylene biosynthesis, contributes to the resistance of rice to blast fungus.Plant Physiol. 155:502-514. 
Shah, F., Huang, J., Cui, K., Nie, L., Shah, T., Chen, C., and Wang, K. 2011. Impact of high-temperature on rice plants and its trait related to tolerance. J. Agri Sci. 149:545-549.

Sheikh, A. H., Raghuram, B., Jalmi, S. K., Wankhede, D. P., Singh, P., and Sinha, A. K. 2013. Interaction between two rice mitogen activated protein kinases and its possible role in plant defense. BMC Plant Biol. 13:121.

Shimizu, T., Lin, F., Hasegawa, M., Okada, K., Nojiri, H., and Yamane, H. 2012. Phytoalexin Sakuranetin in Rice Enzyme in Biosynthesis of Flavonoid Naringenin 7- $O$ Methyltransferase, a Key Enzyme in Biosynthesis of Flavonoid Phytoalexin Sakuranetin in Rice. J. Biol. Chem. 287:19315-19325.

Shumilina, D. V., Il'ina, A. V., Kulikov, S. N. and Dzhavakhiya, V. G. 2005. Elicitor activity of MF-3 protein from Pseudomonas fl uorescens and combination of MF3-protein with chitosan in different host-pathogen pairs. Adv. Chitin Sci. 8:275-278.

Singh, P., Kuo, Y. C., Mishra, S., Tsai, C. H., Chien, C. C., Chen, C. W., DesclosTheveniau, M., Chu, P. W., Schulze, B., Chinchilla, D., Boller, T., and Zimmerli, L. 2012. The lectin receptor kinase-vi.2 is required for priming and positively regulates Arabidopsis pattern-triggered immunity. Plant Cell 24:1256-1270.

Suzuki, N., Bajad, S., Shuman, J., Shulaev, V., and Mittler, R. 2008. The transcriptional co-activator MBF1c is a key regulator of thermotolerance in Arabidopsis thaliana. J. Biol. Chem. 283:9269-9275.

Torres, M. A., Jones, J. D., and Dangl, J. L. 2006. Reactive oxygen species signaling in response to pathogens. Plant Physiol 141:373-378.

Umemura, K., Satou, J., Iwata, M., Uozumi, N., Koga, J., Kawano, T., Koshiba, T., Anzai, H., and Mitomi, M. 2009. Contribution of salicylic acid glucosyltransferase, OsSGT1, to chemically induced disease resistance in rice plants. The Plant J. 57:463-472.

Ueno, M., Kumura, Y., Ueda, K., Kihara, J., and Arase S. 2011. Indole derivatives enhance resistance against the rice blast fungus Magnaporthe oryzae. J. Gen. Plant Pathol. 77:209-213.

Webb, K. M., Ona, I., Bai, J., et al. 2011. A benefit of high temperature: increased effectiveness of a rice bacterial blight disease resistance gene. New Physiol. 185:568-576.

Wei, T., Ou, B., Li, J., Zhao, Y., Guo, D., et al. 2013. Transcriptional Profiling of Rice Early Response to Magnaporthe oryzae Identified OsWRKYs as Important Regulators in Rice Blast Resistance. PLoS ONE 8:e59720.

Wrzaczek, M., Brosché, M., Salojärvi, J., Kangasjärvi, S., Idänheimo, N., Mersmann, S., Robatzek, S., Karpiński, S., Karpińska, B., and Kangasjärvi, J. 2010. Transcriptional regulation of the CRK/DUF26 group of receptor-like protein kinases by ozone and plant hormones in Arabidopsis. BMC Plant Biol. 10:95. 
Xiao, S. Y., Brown, S., Patrick, E., Brearley, C., and Turner, J. G. 2003. Enhanced transcription of the Arabidopsis disease resistance genes RPW8.1 and RPW8.2 via a salicylic acid-dependent amplification circuit is required for hypersensitive cell death. Plant Cell 15:33-4.

Yan, G. P., and Chen, X. M. 2008. Identification of a quantitative trait locus for hightemperature adult-plant resistance against Puccinia striiformis f. sp hordei in 'Bancroft' barley. Phytopathol. 98:120-127.

Yang, S. H., Hua, J. 2004. A haplotype-specific resistance gene regulated by BONZAI1 mediates temperature-dependent growth control in Arabidopsis. Plant Cell 16:1060-1071.

You, M. K., Shin, H. Y., Kim, Y. J., Ok, S. H., Cho, S. K., Jeung, J. U., Yoo, S. D., Kim, J. K., and Shin, J. S. 2010. Novel bifunctional nucleases, OmBBD and AtBBD1, are involved in abscisic acid-mediated callose deposition in Arabidopsis. Plant Physiol. 152:1015-1029.

Young, M. D., Wakefield, M. J., Smyth, G. K., and Oshlack, A. 2010. Gene ontology analysis for RNA-seq: accounting for selection bias. Genome Biol. 11:R14.

Zhang, S., Chen, C., Li, L., Meng, L., Singh, J., Jiang, N., Deng, X-W., He, Z.H., and Lemaux, P.G. 2005. Evolutionary Expansion, Gene Structure, and Expression of the Rice Wall-Associated Kinase Gene Family. Plant Physiol. 139:1107-1124.

Zhao, N., Guan, J., Ferrer, J. L., Engle, N., Chern, M., Ronald, P., Tschaplinski, T. J., and Chen, F. 2010. Emission, biosynthesis and regulation of insect-induced methyl salicylate and methyl benzoate from rice. Plant Physiol. Biochem. 48:279-287. 


\section{Figures}

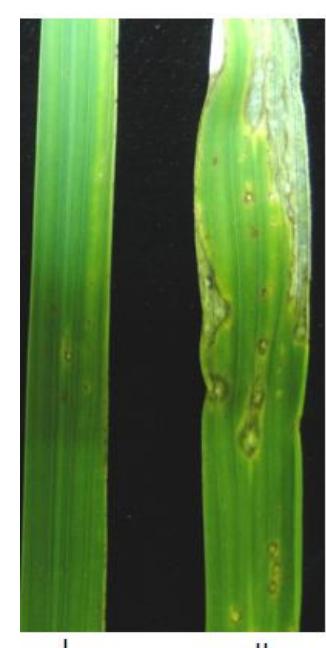

II

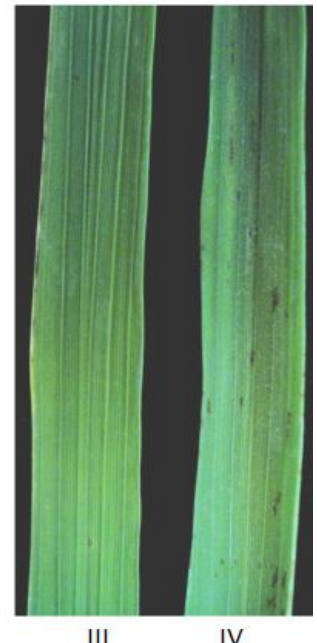

III

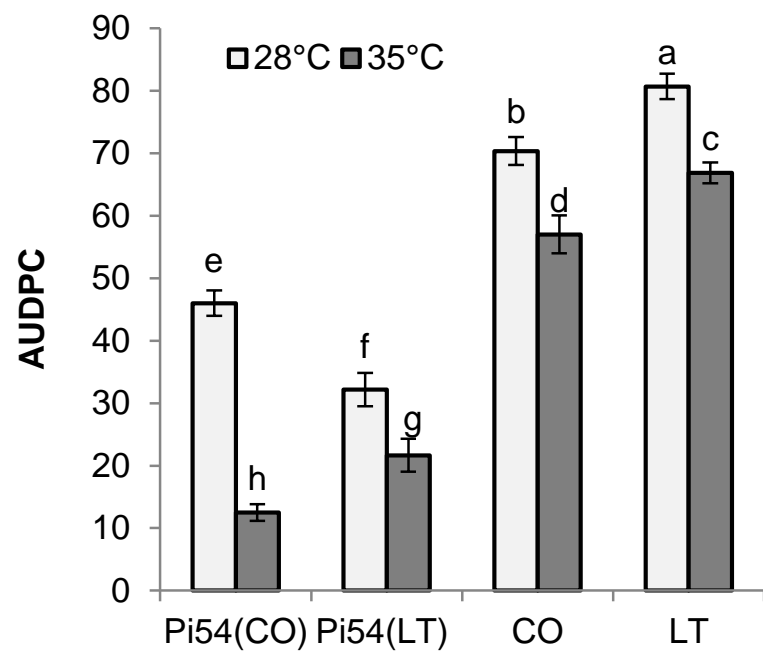

Genotypes
Figure 2.1a: Disease phenotypes of $M$.

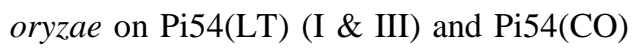
(II \& IV) at $28^{\circ} \mathrm{C}$ and $35^{\circ} \mathrm{C}, 5 \mathrm{dpi}$. I and II represent disease phenotype at $28^{\circ} \mathrm{C}$, and III and IV represent disease phenotype in plants pre-exposed to $35^{\circ} \mathrm{C}$ prior to inoculation at $28^{\circ} \mathrm{C}$.
Figure 2.1b: Disease progress of $M$. oryzae on $\mathrm{Pi} 54(\mathrm{CO})$ and Pi54(LT) carrying R gene Pi54, and their background parents LTH (LT) and CO39 (CO) at $28^{\circ} \mathrm{C}$ and $35^{\circ} \mathrm{C}$. Same letters above standard error bars indicate no significant difference $(\mathrm{P}<0.05)$. The genetic backgrounds are indicated as LT and CO.
A

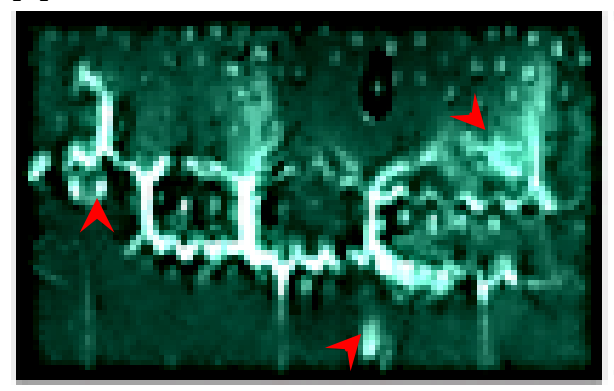

B

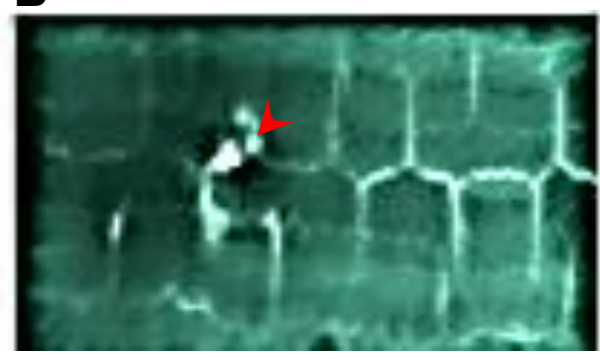

C

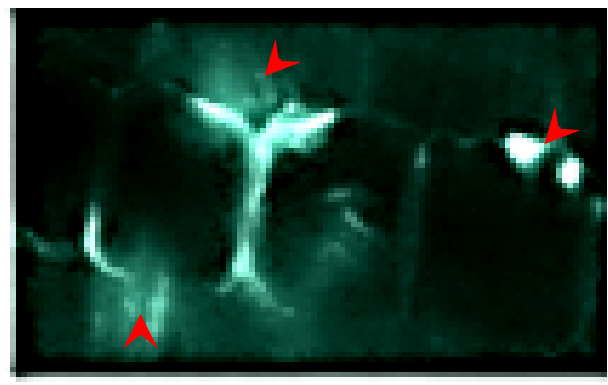

D

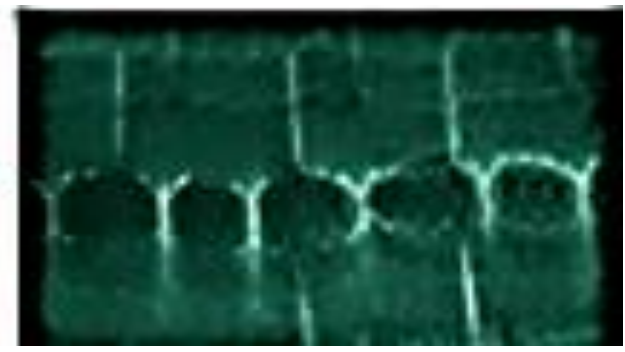

Figure 2.2: Callose deposition due to anilin blue staining of epidermal cells of LT (A and B) and CO (C and D) $48 \mathrm{hrs}$ after inoculation with $M$. oryzae. A and $\mathrm{C}$ represent plants grown at $35^{\circ} \mathrm{C}$ for 7 days before inoculation, and $\mathrm{B}$ and $\mathrm{D}$ plants grown at $28^{\circ} \mathrm{C}$ before inoculation. Callose deposits appear more pronounced at $35^{\circ} \mathrm{C}$ compared to $28^{\circ} \mathrm{C}$ in both genetic backgrounds. LT shows more intense cell wall florescence than $\mathrm{CO}$ at both temperatures. 


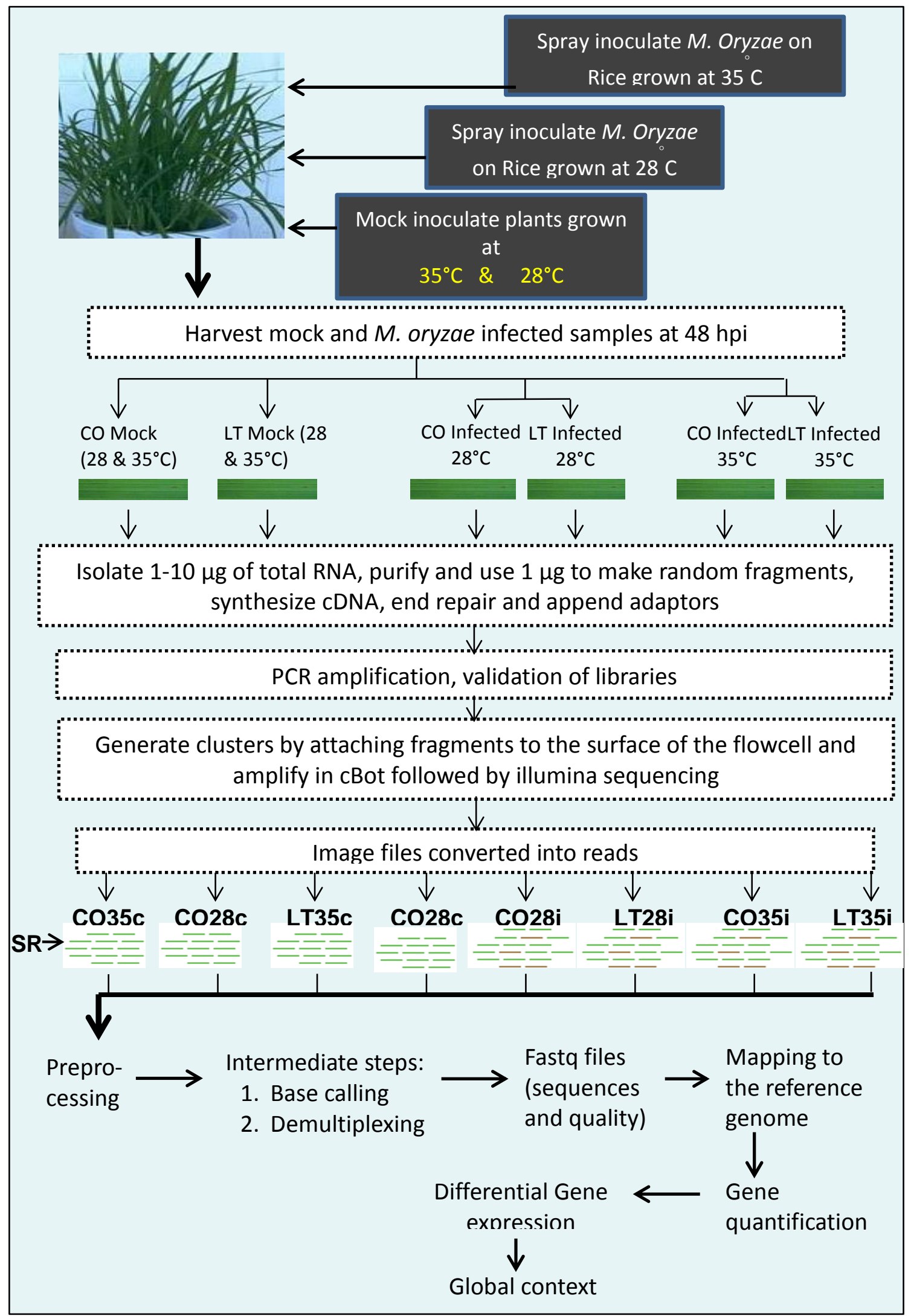

Figure 2.3a: Overview of the steps involved in the mRNA isolation, illumina sequencing, preprocessing, mapping, and analysis of illumina reads and subsequent gene expression data. LT and CO represent genetic backgrounds carrying Pi54 and exposed to $35^{\circ} \mathrm{C}$ and $28^{\circ} \mathrm{C}$. Both genetic backgrounds were subjected to mock and $M$. oryzae inoculation. The mRNA reads were mapped to the Ensembl cDNA release 16 for Oryza sativa. SR-sequenced reads 


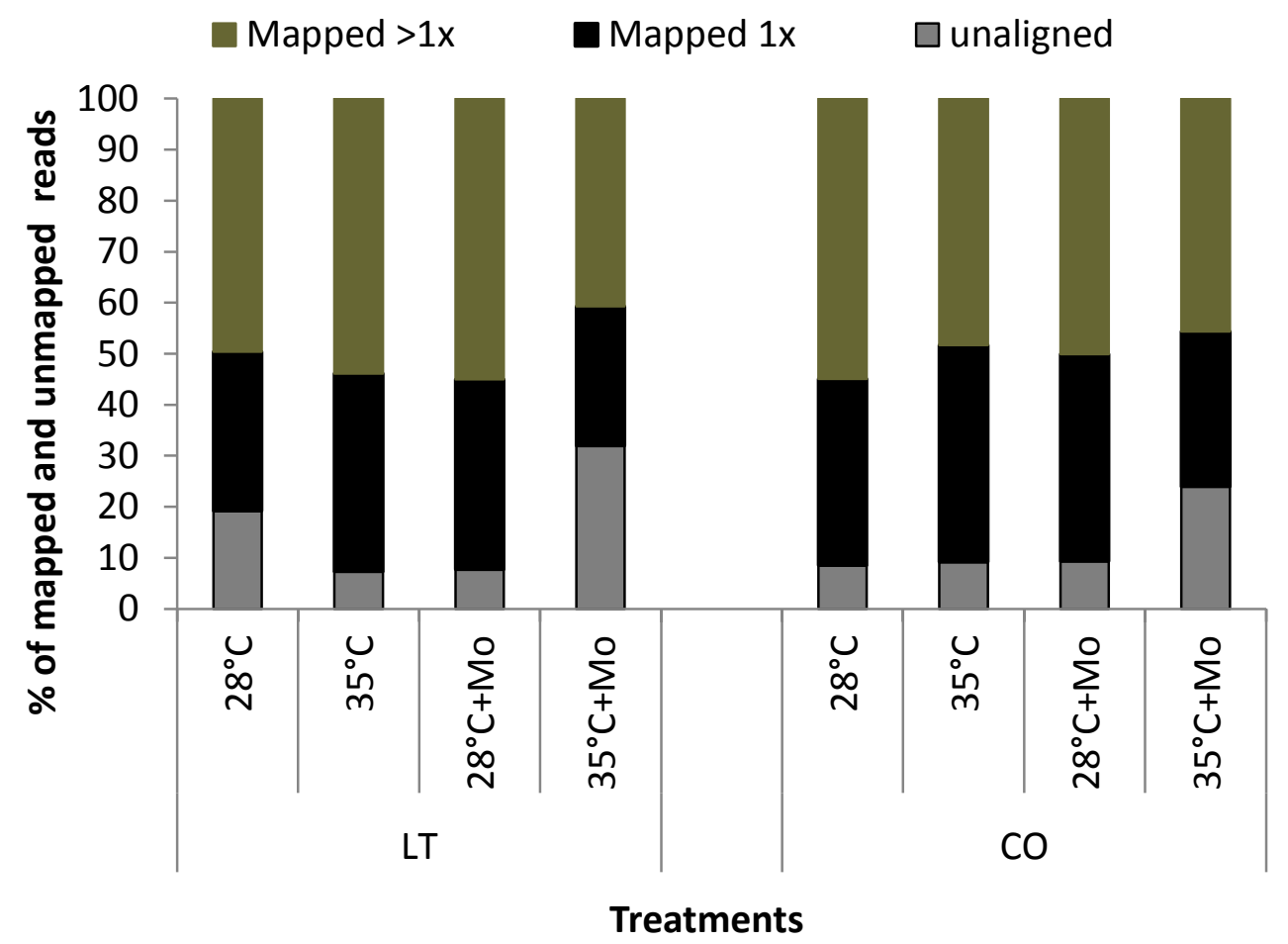

Figure 2.3b: Summary of the alignment statistics of the read mapping onto the reference genome, Ensembl cDNA release 16 for Oryza sativa, is presented in clustered stacked columns. Grey represents percentage of unmapped reads, black represents unique reads and green represents non unique reads. LT and CO are the genetic backgrounds carrying Pi54 and exposed to $35^{\circ} \mathrm{C}$ and $28^{\circ} \mathrm{C}$. Mo: M. oryzae infection. 


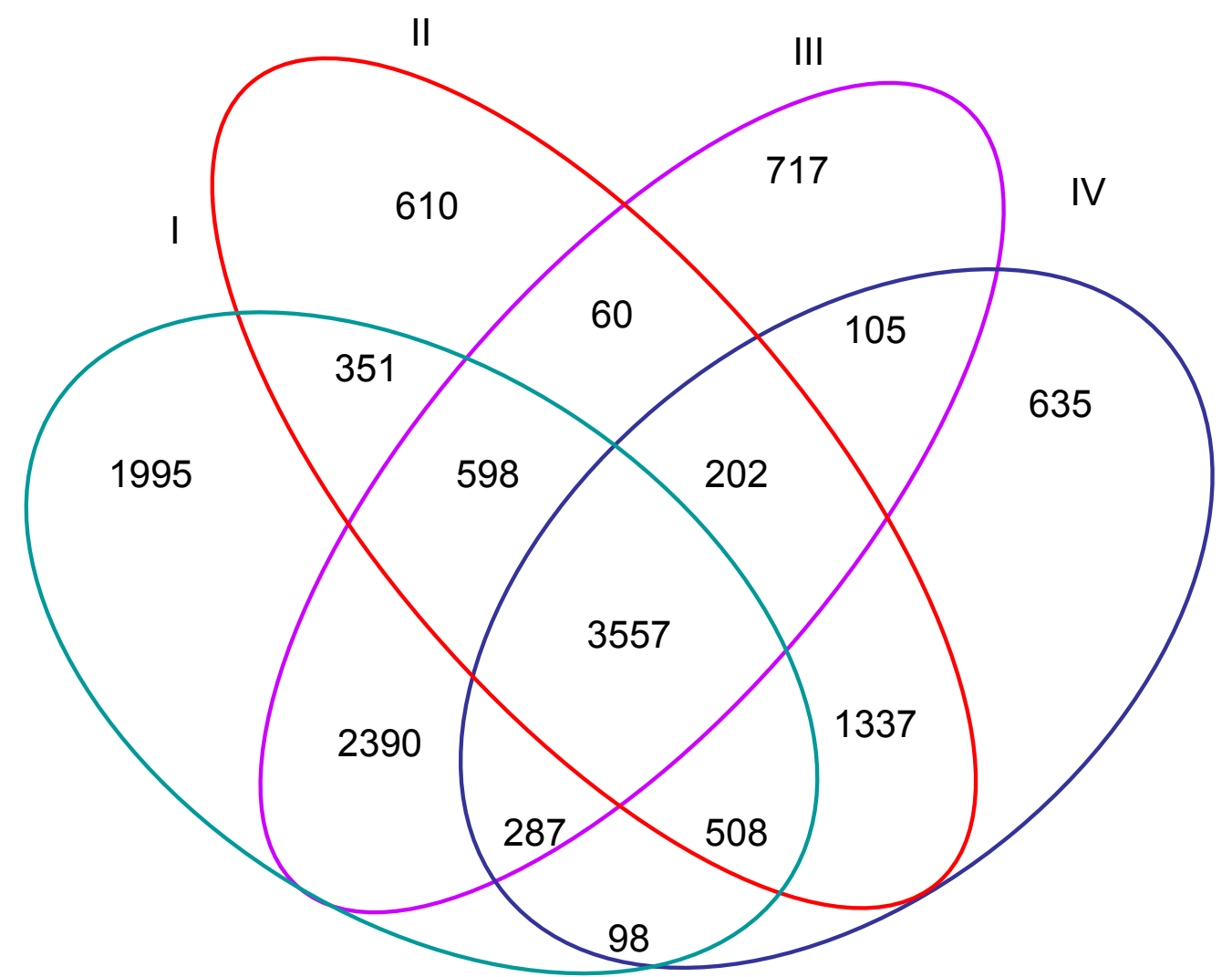

Figure 2.4a: Venn diagram analysis of the differentially expressed genes (fold change 4; FDR, 0.05) in (I) $\mathrm{CO}+28^{\circ} \mathrm{C}+\mathrm{Mo}$, (II) $\mathrm{LT}+28^{\circ} \mathrm{C}+\mathrm{Mo}$, (III) $\mathrm{CO}+35^{\circ} \mathrm{C}+\mathrm{Mo}$ and (IV) $\mathrm{LT}+35^{\circ} \mathrm{C}+\mathrm{Mo}$.

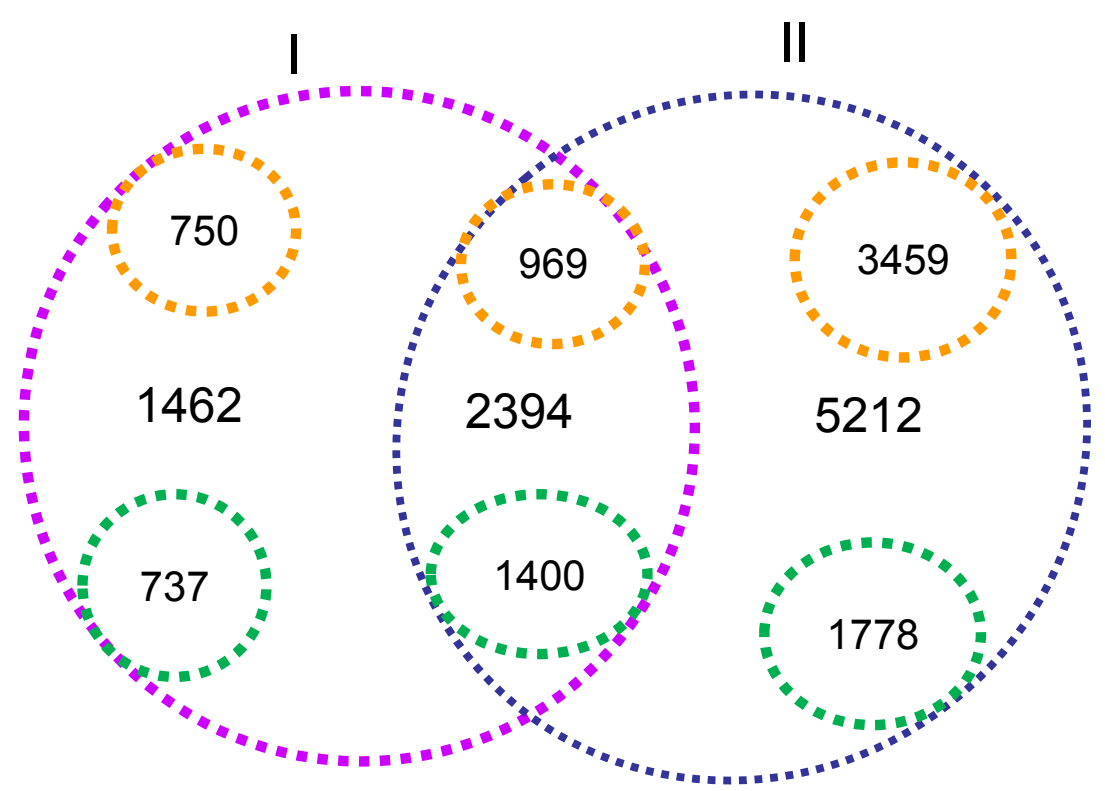

Figure 2.4b: Venn diagram analysis of the differentially expressed genes (fold change 4; FDR, 0.05) in (I) $\mathrm{CO}+35^{\circ} \mathrm{C}$, (II) $\mathrm{LT}+35^{\circ} \mathrm{C}$. The numbers in the orange circles represent the specific and shared up regulated genes in the respective treatments while those in the green circles represent specific and shared down regulated genes. 
Chapter 2: Rice NILs carrying Pi54 in LTH and Co39 backgrounds confer resistance to Magnaporthe oryzae at $35^{\circ} \mathrm{C}$ but show contrasting reactions at $28^{\circ} \mathrm{C}$

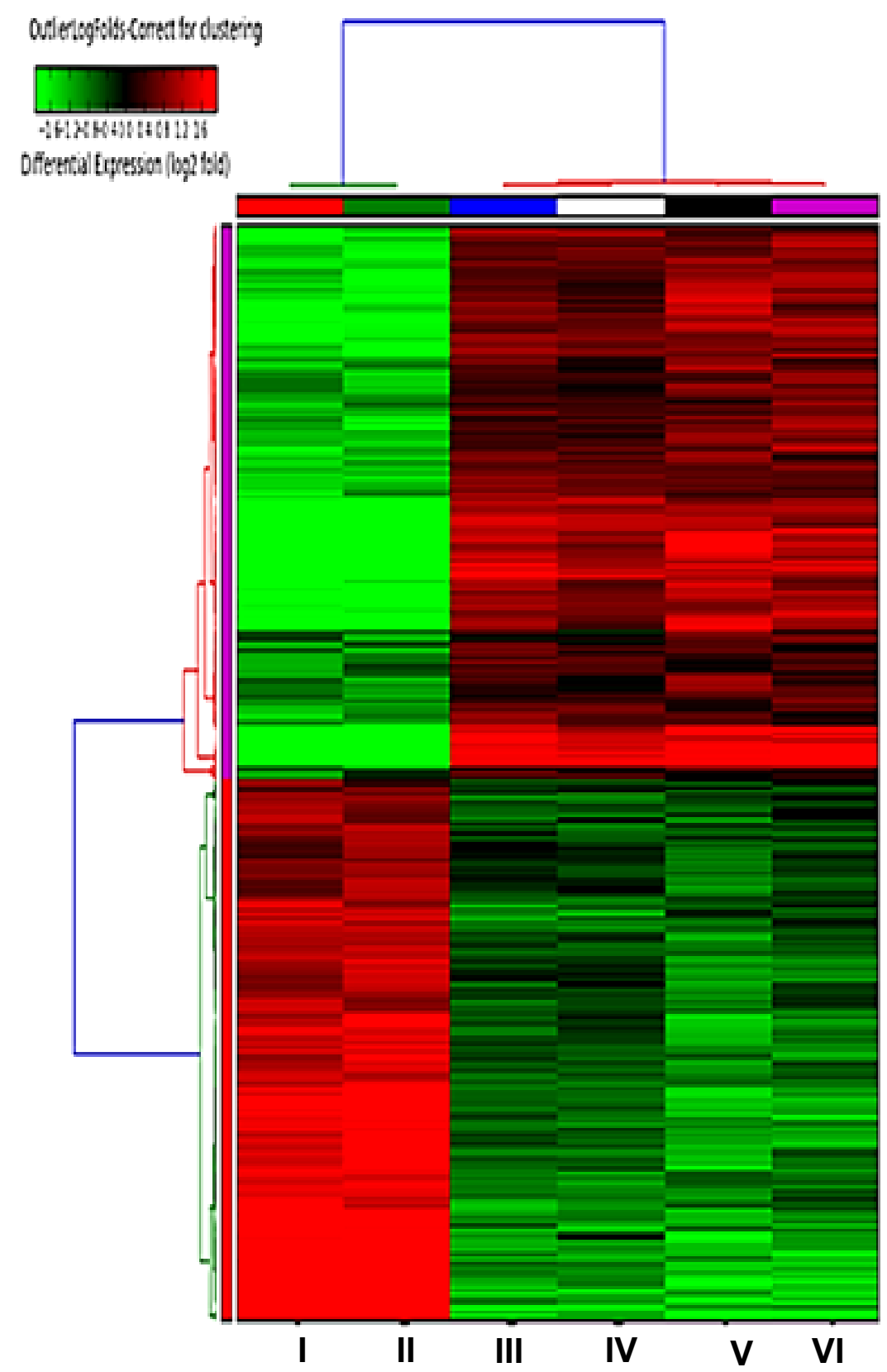

Figure 2.4c: Heat map showing the gene expression clusters and sample clusters. The color bar represents the $\log 2$ of fold change values compared to mock, ranging from green to red. Samples I, II, III, IV, $\mathrm{V}$ and VI represent $\mathrm{CO}+35^{\circ} \mathrm{C}, \mathrm{LT}+35^{\circ} \mathrm{C}, \mathrm{LT}+28^{\circ} \mathrm{C}+\mathrm{Mo}, \mathrm{LT}+35^{\circ} \mathrm{C}+\mathrm{Mo}, \mathrm{CO}+28^{\circ} \mathrm{C}+\mathrm{Mo}$ and $\mathrm{CO}+35^{\circ} \mathrm{C}+\mathrm{Mo}$, respectively. 
Chapter 2: Rice NILs carrying Pi54 in LTH and Co39 backgrounds confer resistance to Magnaporthe oryzae at $35^{\circ} \mathrm{C}$ but show contrasting reactions at $28^{\circ} \mathrm{C}$

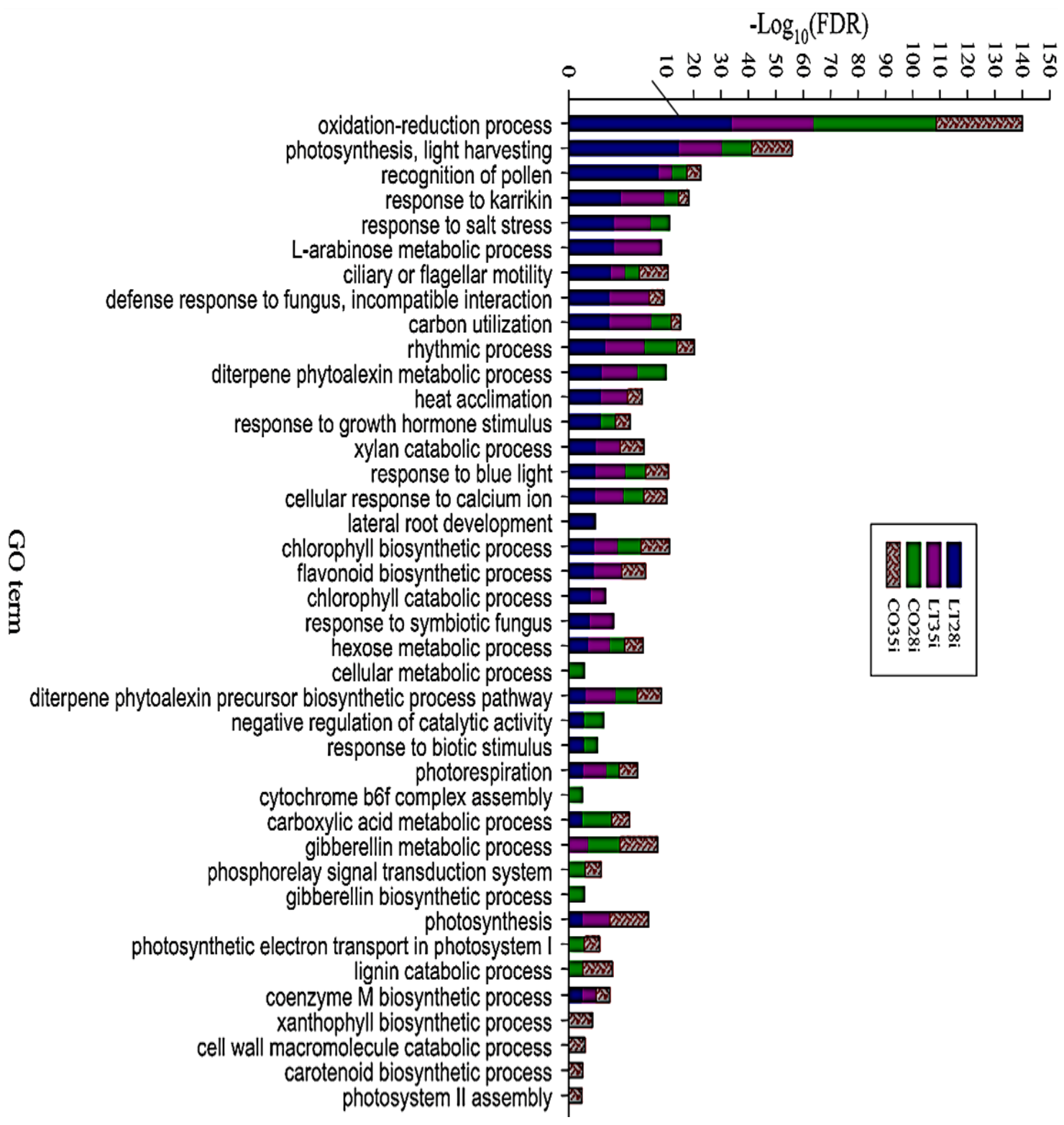

Figure 2.5: Significant Gene Ontology (GO) enriched terms from differentially expressed genes in infected samples (fold change 4; FDR 0.05); blue bars, $\mathrm{LT}+28^{\circ} \mathrm{C}+\mathrm{Mo}$; purple bars, $\mathrm{LT}+35^{\circ} \mathrm{C}+\mathrm{Mo}$; green bars, $\mathrm{CO}+28^{\circ} \mathrm{C}+\mathrm{Mo}$; red stripe bars, $\mathrm{CO}+35^{\circ} \mathrm{C}+\mathrm{Mo}$. 
A

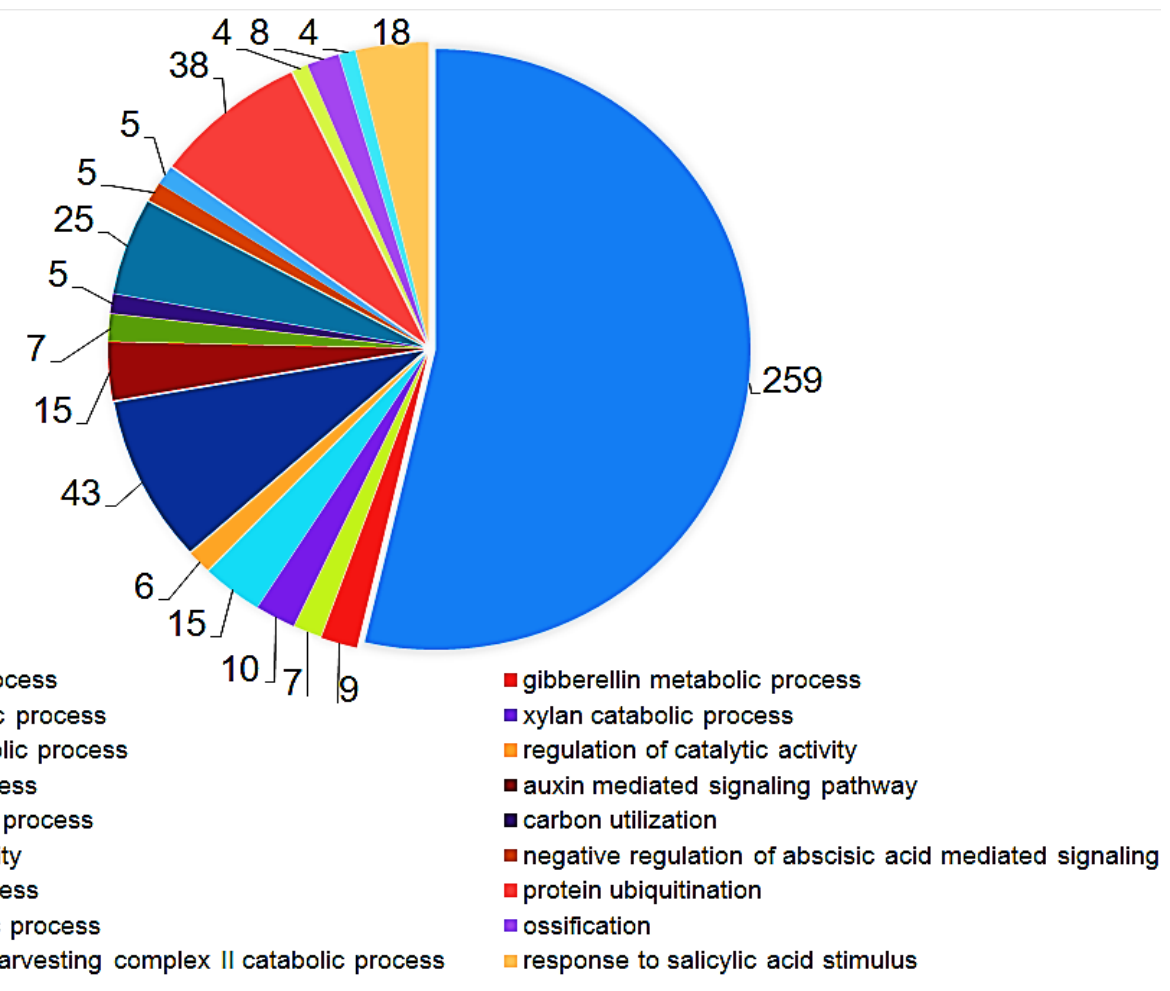

B

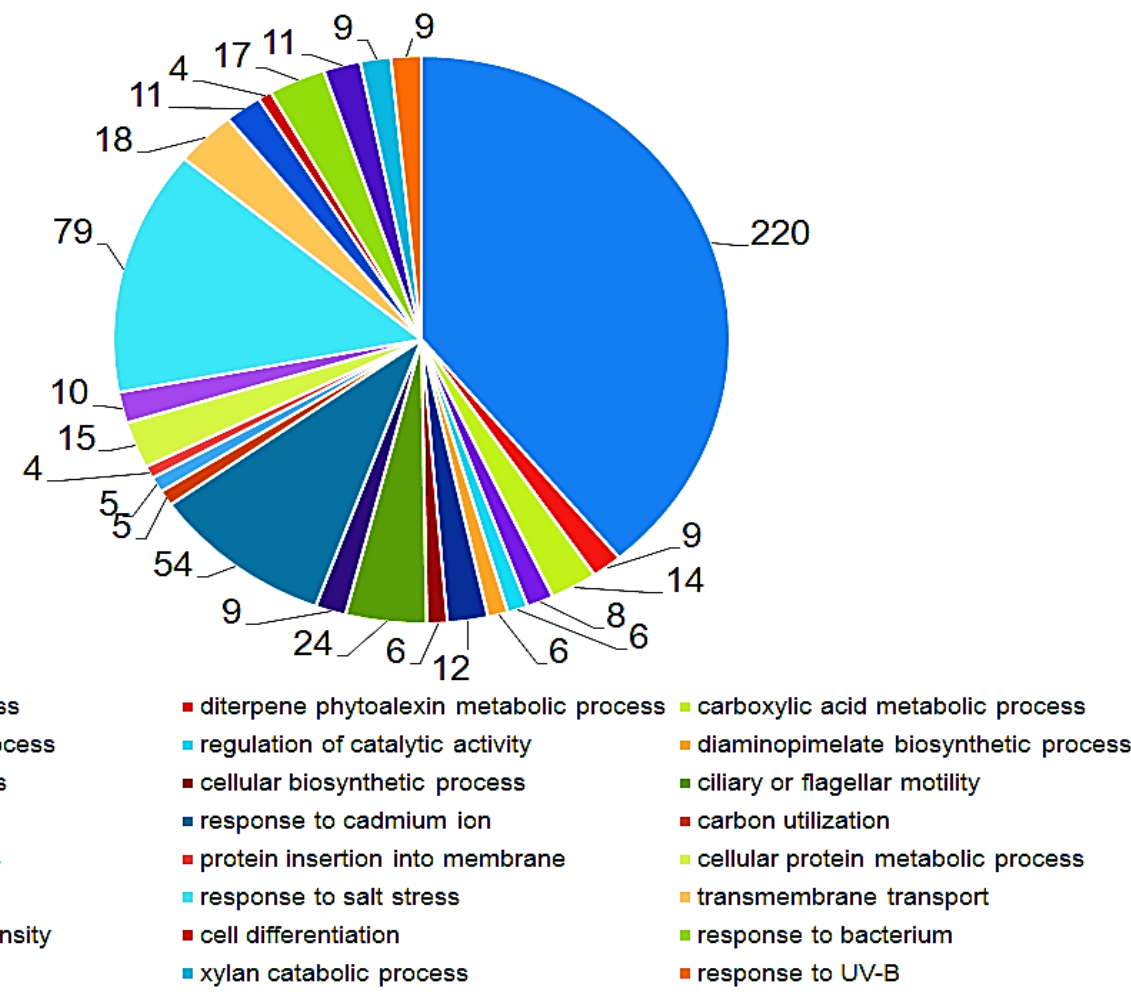

Figure 2.6: Classification of up regulated DEGs into biological processes. (A) experiment comparing RNA derived from $\mathrm{CO}+35^{\circ} \mathrm{C}+\mathrm{Mo}$ to that derived from its control $\left(\mathrm{CO}+28^{\circ} \mathrm{C}\right)$ and $\mathrm{B}$ comparing sample $\mathrm{LT}+35^{\circ} \mathrm{C}$ + Mo to its control $\left(\mathrm{LT}+28^{\circ} \mathrm{C}\right)$. Only significantly enriched $\mathrm{GO}$ terms are presented based on over represented $\mathrm{P}$-value of $<0.05$. The numbers represent the fraction of genes enriching the specific GO term represented in the legend. 


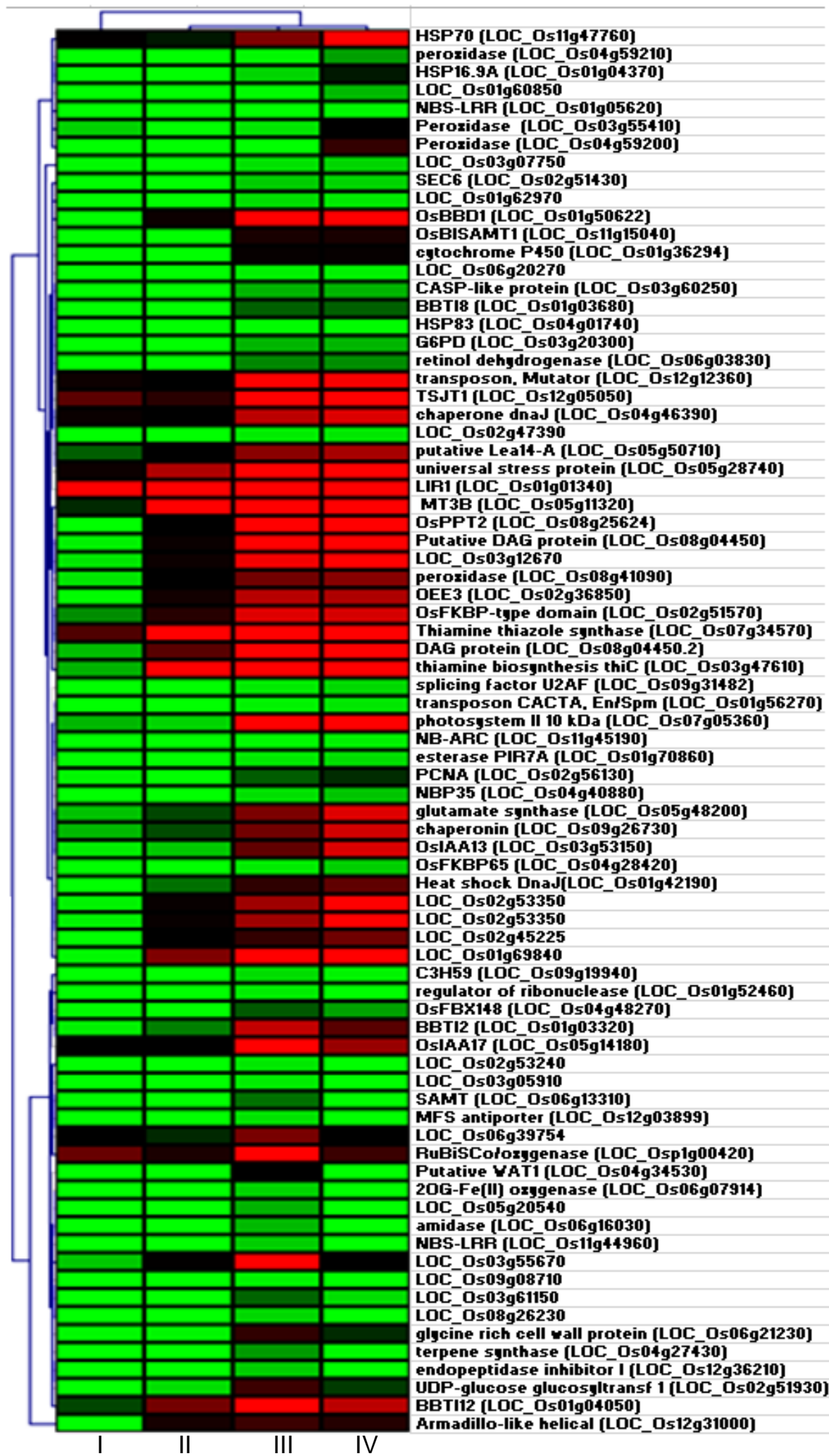

Figure 2.7: Expression profile matrix of highly induced genes in $\mathrm{CO}$ relative to other treatments in response to only high tempretature, made by hierarchical clustering. The treatments I-IV indicated below the cluster analysis represent $\mathrm{LT}+28^{\circ} \mathrm{C}, \mathrm{CO}+28^{\circ} \mathrm{C}, \mathrm{CO}+35^{\circ} \mathrm{C}$ and $\mathrm{LT}+35^{\circ} \mathrm{C}$. On the left side of the cluster analyses the cluster dendogram is shown. 


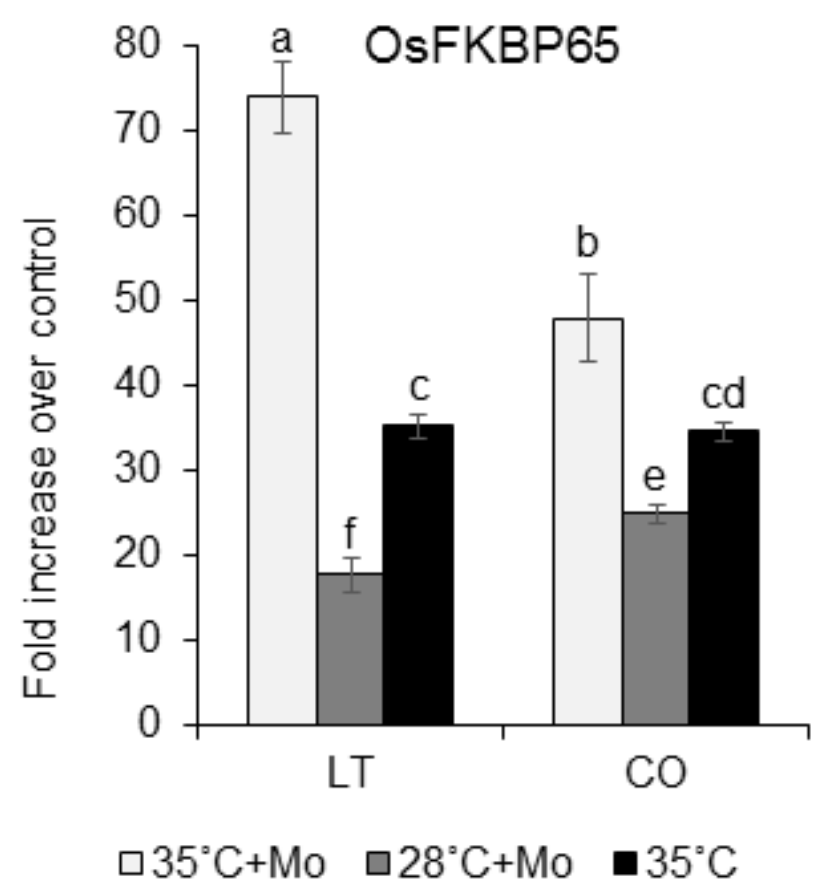

Figure 2.8: The effect of temperature, on transcript levels of OsFKBP65 in LT and Co genetic backgrounds. The samples were taken at 48 hours after inoculation.. Mock and high temperature samples were taken at 0 hpi. Data are presented as mean fold increase over control \pm standard deviation. Same letters above standard error bars indicate no significant difference at $\mathrm{P}<0.05$.

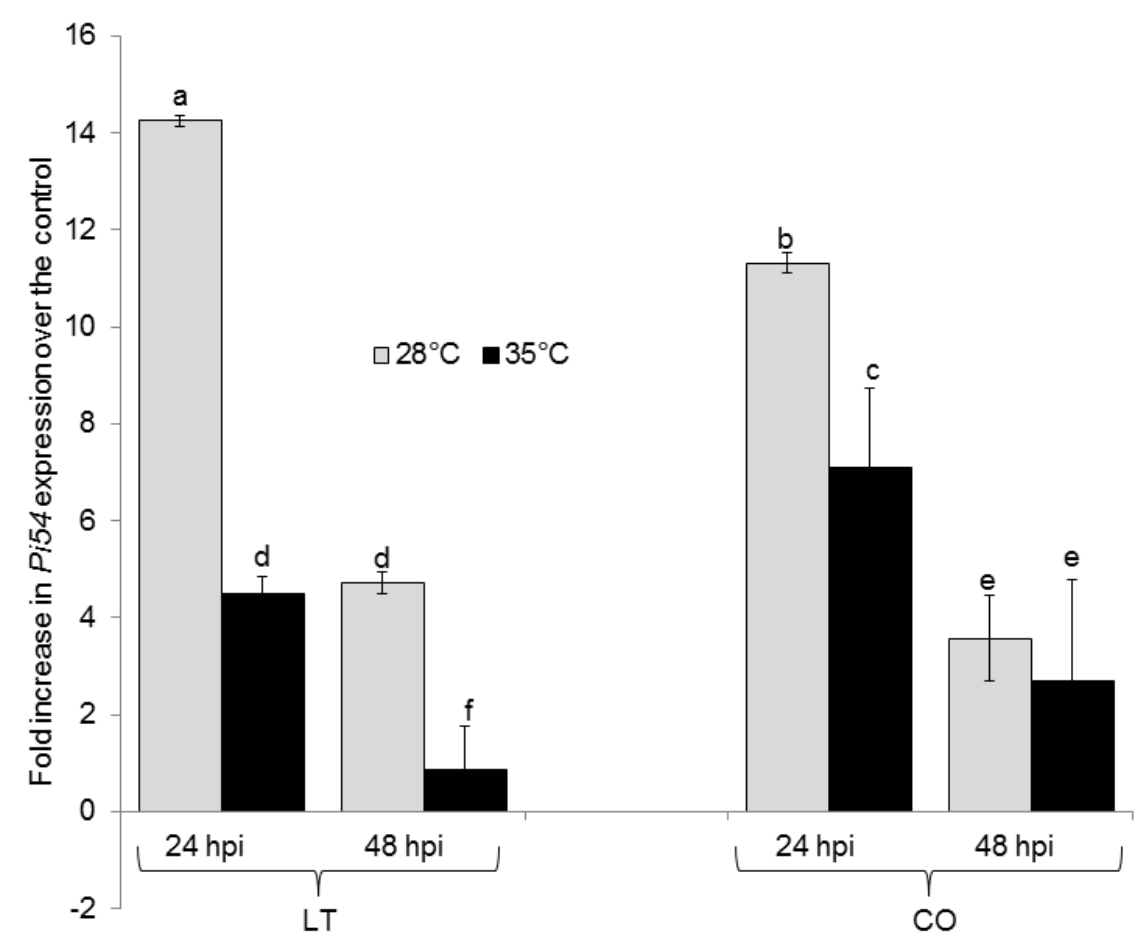

Figure 2.9: The effect of temperature, on transcript levels of Pi54 in LT and Co genetic backgrounds. The samples were taken at 24 and 48 hours after inoculation (hpi). Mock and high temperature samples were taken at 0 hpi. Data are presented as mean fold increase over control \pm standard deviation. Same letters above standard error bars indicate no significant difference at $\mathrm{P}<0.05$. 

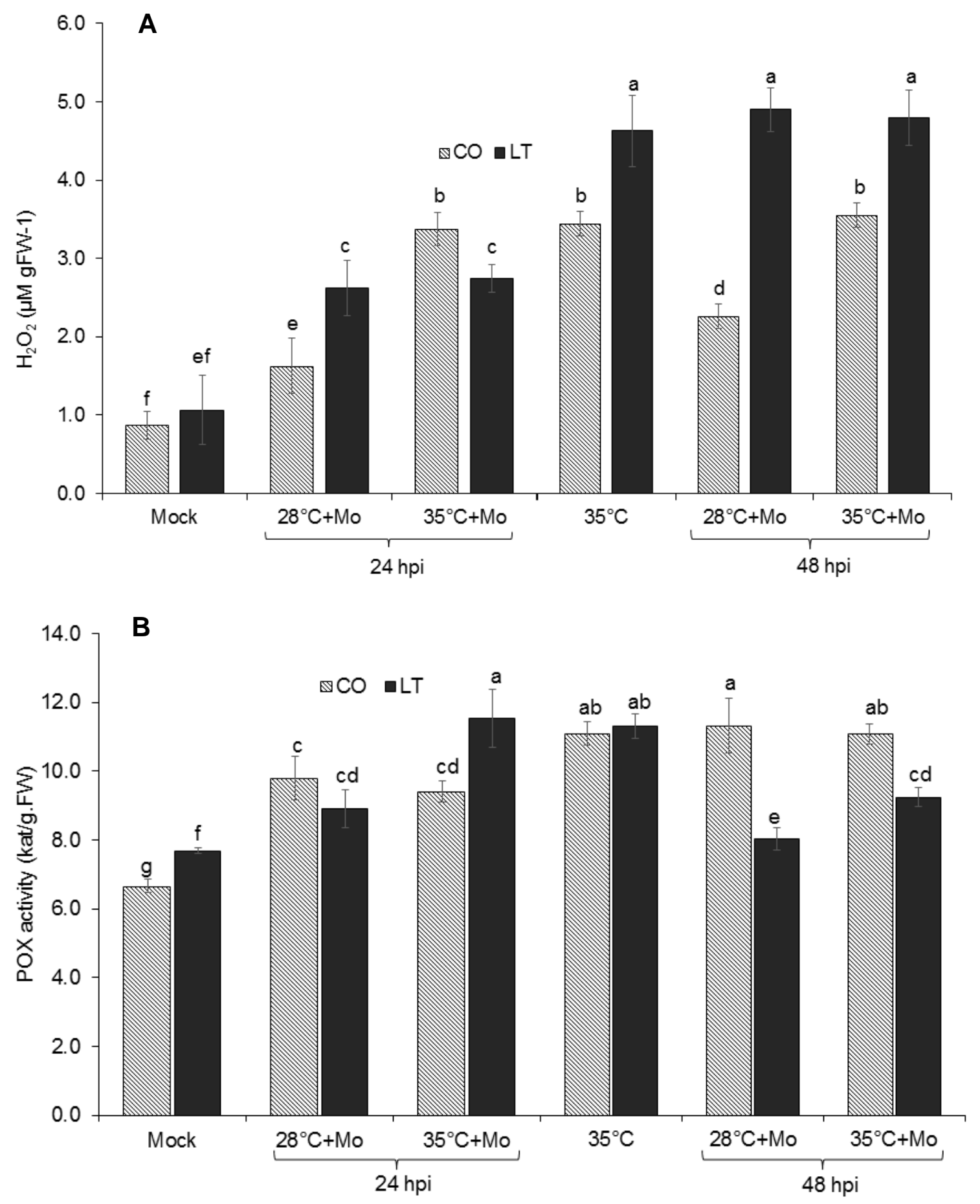

Figure 2.10: Hydrogen peroxide $\left(\mathrm{H}_{2} \mathrm{O}_{2}\right)$ quantification in micromoles per gram (A) and POX activity measure in mkatal (kat) per gram (B) of fresh leaf tissue weight (FW) collected from healthy (Mock), M. oryzae inoculated $\left(28^{\circ} \mathrm{C}+\mathrm{Mo}\right)$, high temperature $\left(35^{\circ} \mathrm{C}\right)$, and high temperature plus $M$. oryzae inoculated $\left(35^{\circ} \mathrm{C}+\mathrm{Mo}\right)$. The samples were taken at 24 and 48 hours after inoculation (hpi). Mock and high temperature samples were taken at 0 hpi. One katal (kat) corresponds to the quantity of enzyme activity that transforms $1 \mathrm{~mol} \mathrm{~S}^{-1}$ of substrate. Data are presented as mean \pm standard deviation. Different letters denote significant differences at $\mathrm{P} \leq 0.05$. 


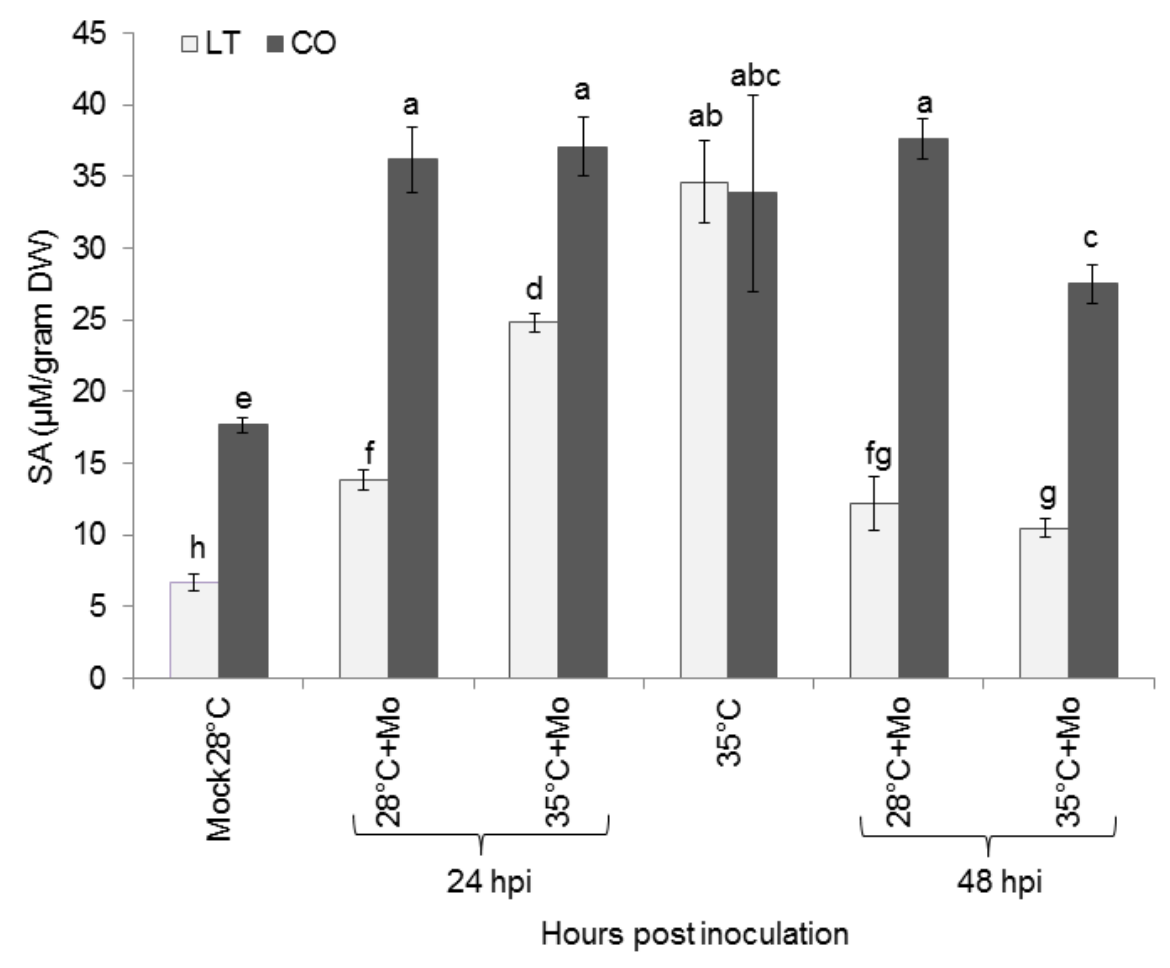

Figure 2.11: Comparison of SA accumulation in $\mathrm{CO}$ and $\mathrm{LT}$ at $28^{\circ} \mathrm{C}$ and $35^{\circ} \mathrm{C}$. Mock $28^{\circ} \mathrm{C}$ and $35^{\circ} \mathrm{C}$, represent mock samples exposed to $28^{\circ} \mathrm{C}$ and $35^{\circ} \mathrm{C}$ without pathogen inoculation. $28^{\circ} \mathrm{C}+\mathrm{Mo}$ and $35^{\circ} \mathrm{C}+\mathrm{Mo}$ represent samples exposed to $28^{\circ} \mathrm{C}$ and $35^{\circ} \mathrm{C}$ prior to $M$. oryzae inoculation. Sampling was done at 24 and 48 hours post inoculation. Different letters denote significant differences at $\mathrm{P} \leq 0.05$.

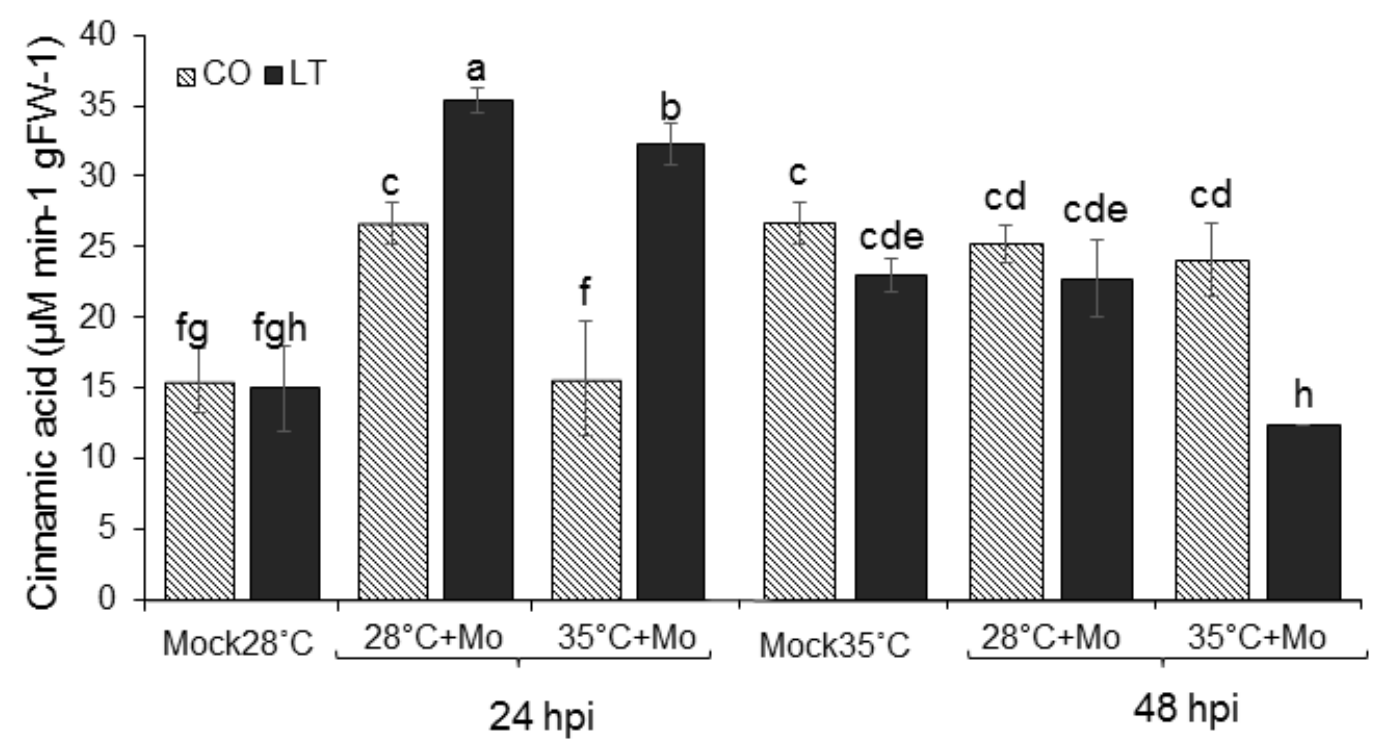

Figure 2.12: PAL activity expressed as micromolar change in cinnamic acid per minute and gram of fresh leaf tissue weight $(\mathrm{FW})$ collected from healthy $\left(\right.$ Mock $\left.28^{\circ} \mathrm{C}\right)$, M. oryzae inoculated $\left(28^{\circ} \mathrm{C}+\mathrm{Mo}\right)$, high temperature (Mock $35^{\circ} \mathrm{C}$ ), and high temperature plus $M$. oryzae inoculated $\left(35^{\circ} \mathrm{C}+\mathrm{Mo}\right)$. The samples were taken at 24 and 48 $\mathrm{h}$ after inoculation (hpi). Mock and high temperature samples were taken at 0 hpi. Data are presented as mean \pm standard deviation. Different letters denote significant differences at $\mathrm{P} \leq 0.05$. 
Chapter 2: Rice NILs carrying Pi54 in LTH and Co39 backgrounds confer resistance to Magnaporthe oryzae at $35^{\circ} \mathrm{C}$ but show contrasting reactions at $28^{\circ} \mathrm{C}$

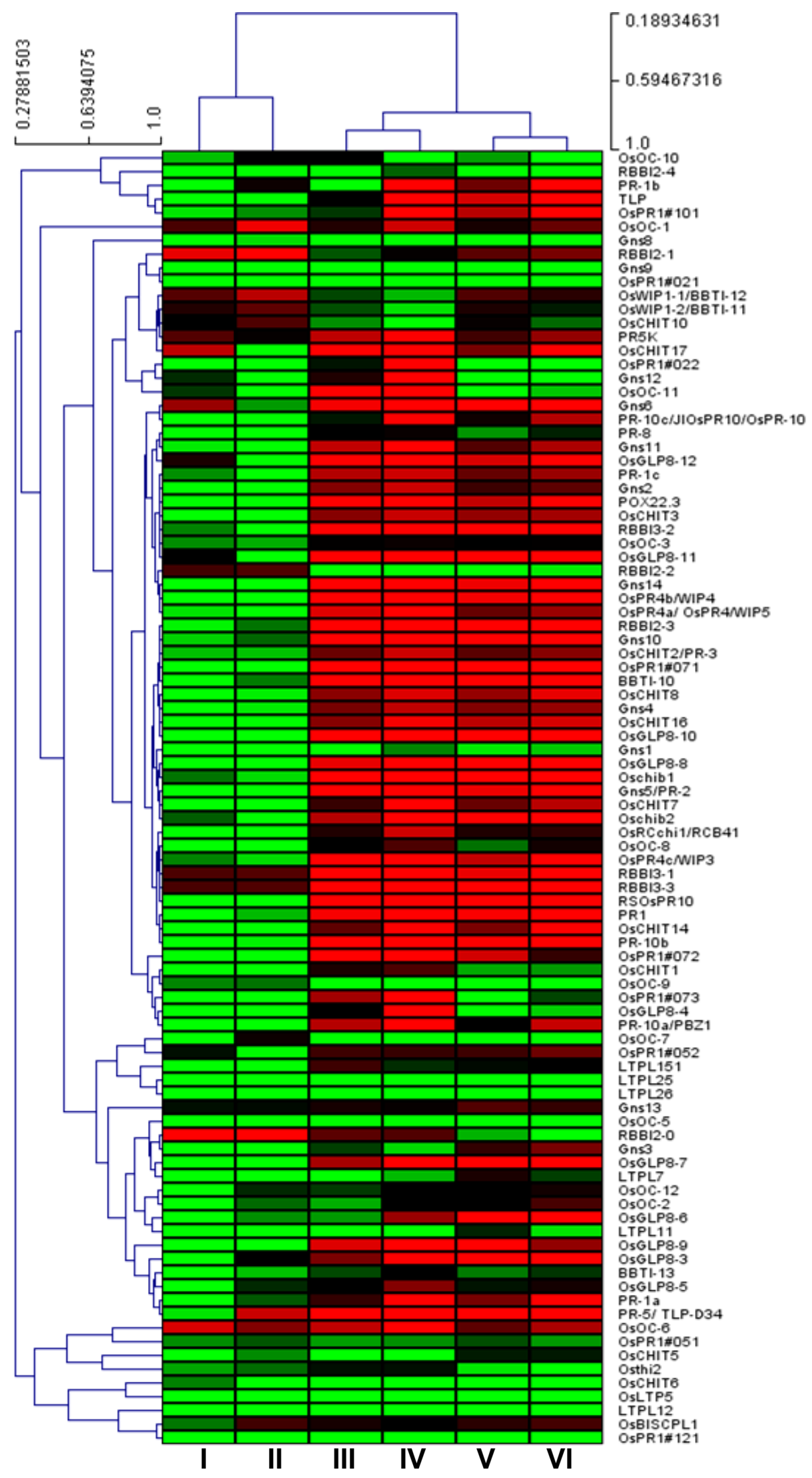

Figure 2.13: Heat map view of the previously reported PR genes differentially expressed in the six treatments I; $\mathrm{CO}+35^{\circ} \mathrm{C}, \mathrm{II} ; \mathrm{LT}+35^{\circ} \mathrm{C}, \mathrm{III} ; \mathrm{CO}+35^{\circ} \mathrm{C}+\mathrm{Mo}, \mathrm{IV} ; \mathrm{CO}+28^{\circ} \mathrm{C}+\mathrm{Mo}, \mathrm{V} ; \mathrm{LT}+35^{\circ} \mathrm{C}+\mathrm{Mo}$, VI; $\mathrm{LT}+28^{\circ} \mathrm{C}+\mathrm{Mo}$. Cluster analysis of expression profiles of the 78 genes. Red indicates higher expression while green represents lower expression. 

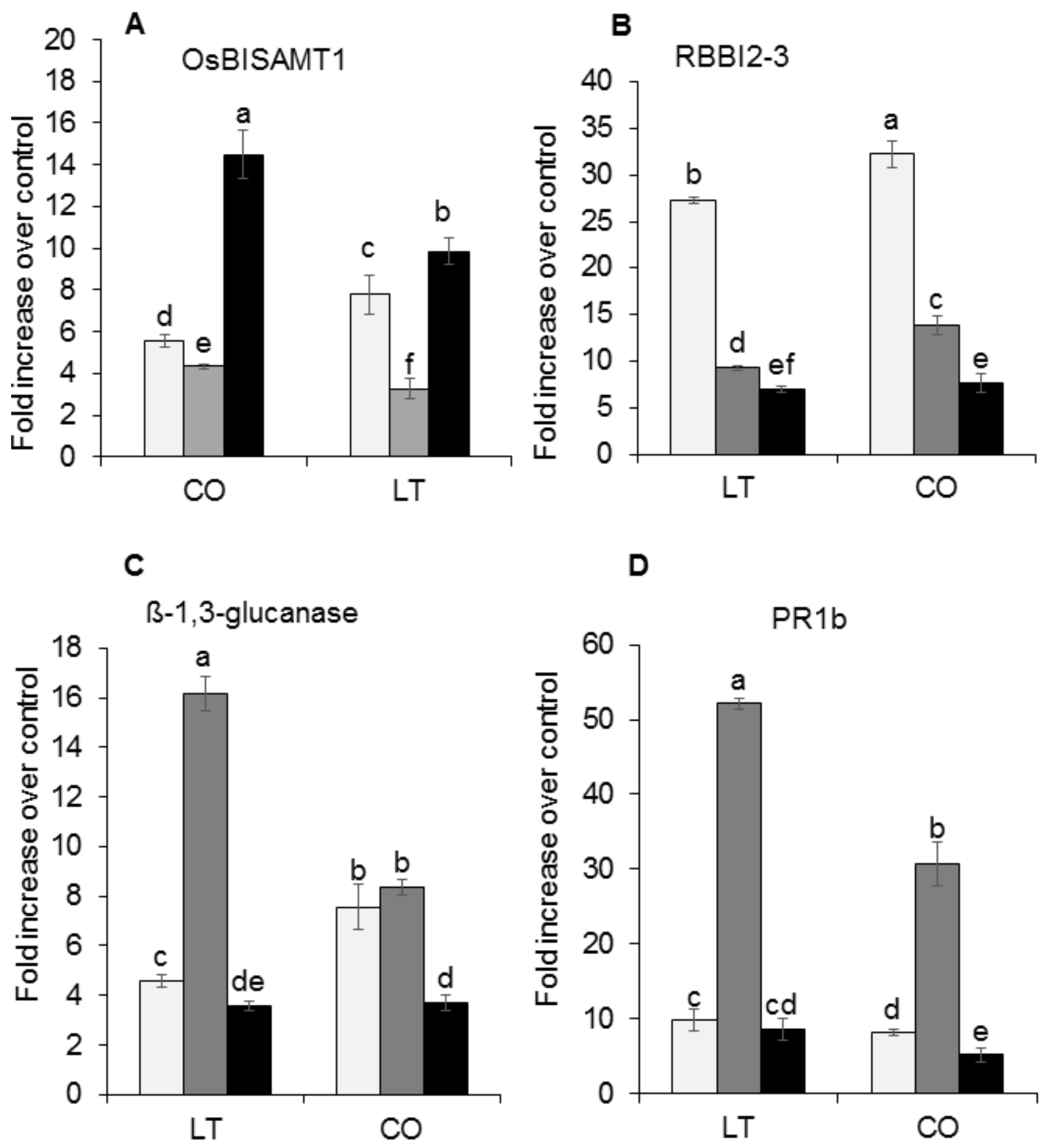
E

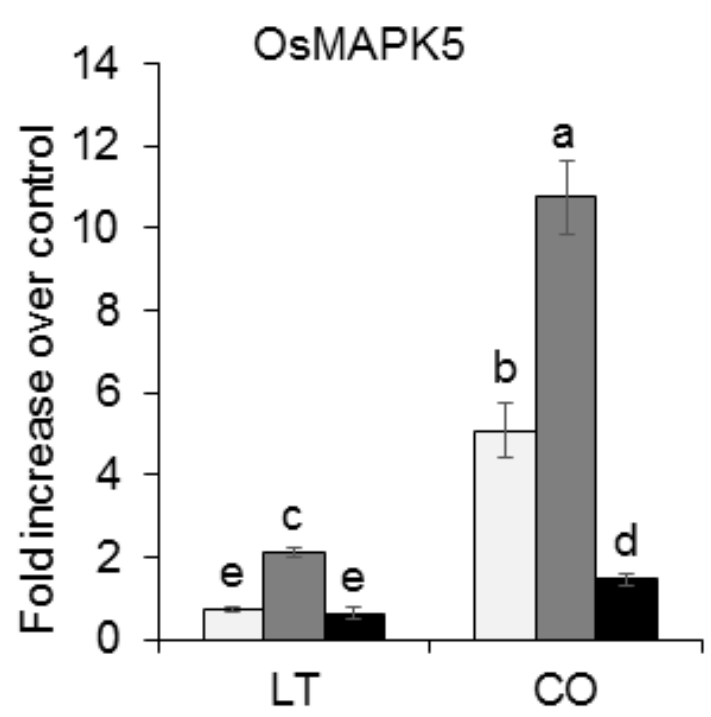

G

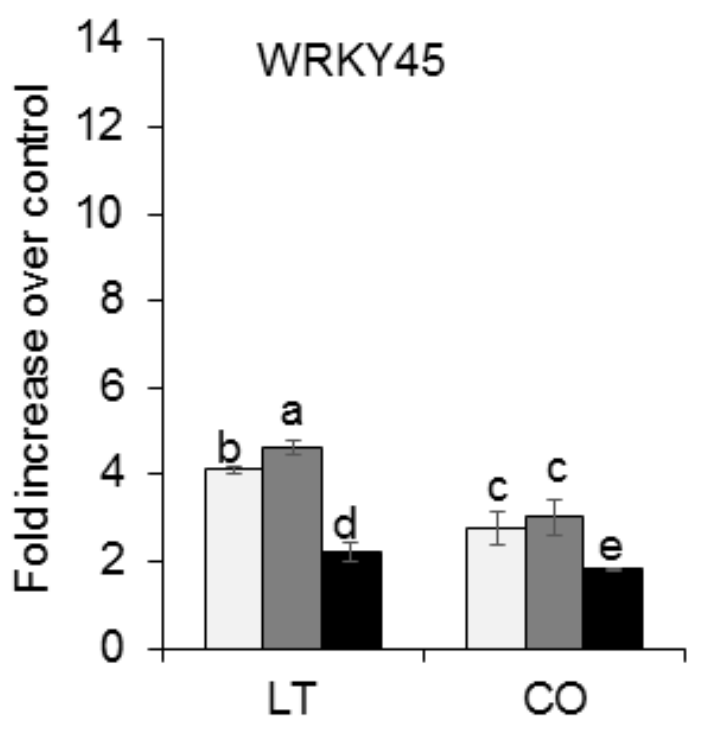

$\mathbf{F}$

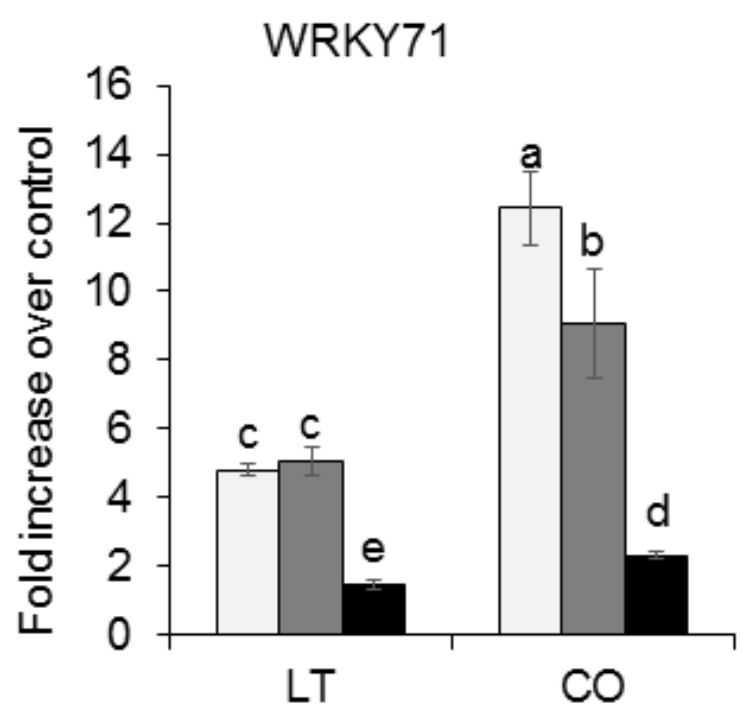

H

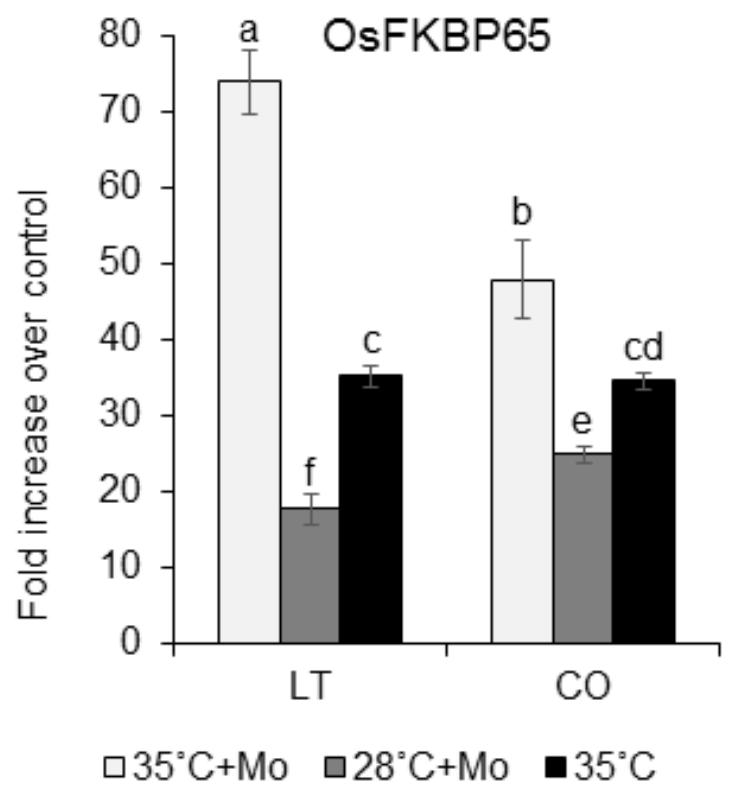

Figure 2.14: Selected differentially expressed genes in all the treatments whose transcription in rice leaves was evaluated by qRT-PCR, relative to mock28 treatment. Bars represent means (three replicates for expression level $\pm \mathrm{SD}$ ). 
Chapter 2: Rice NILs carrying Pi54 in LTH and Co39 backgrounds confer resistance to Magnaporthe oryzae at $35^{\circ} \mathrm{C}$ but show contrasting reactions at $28^{\circ} \mathrm{C}$
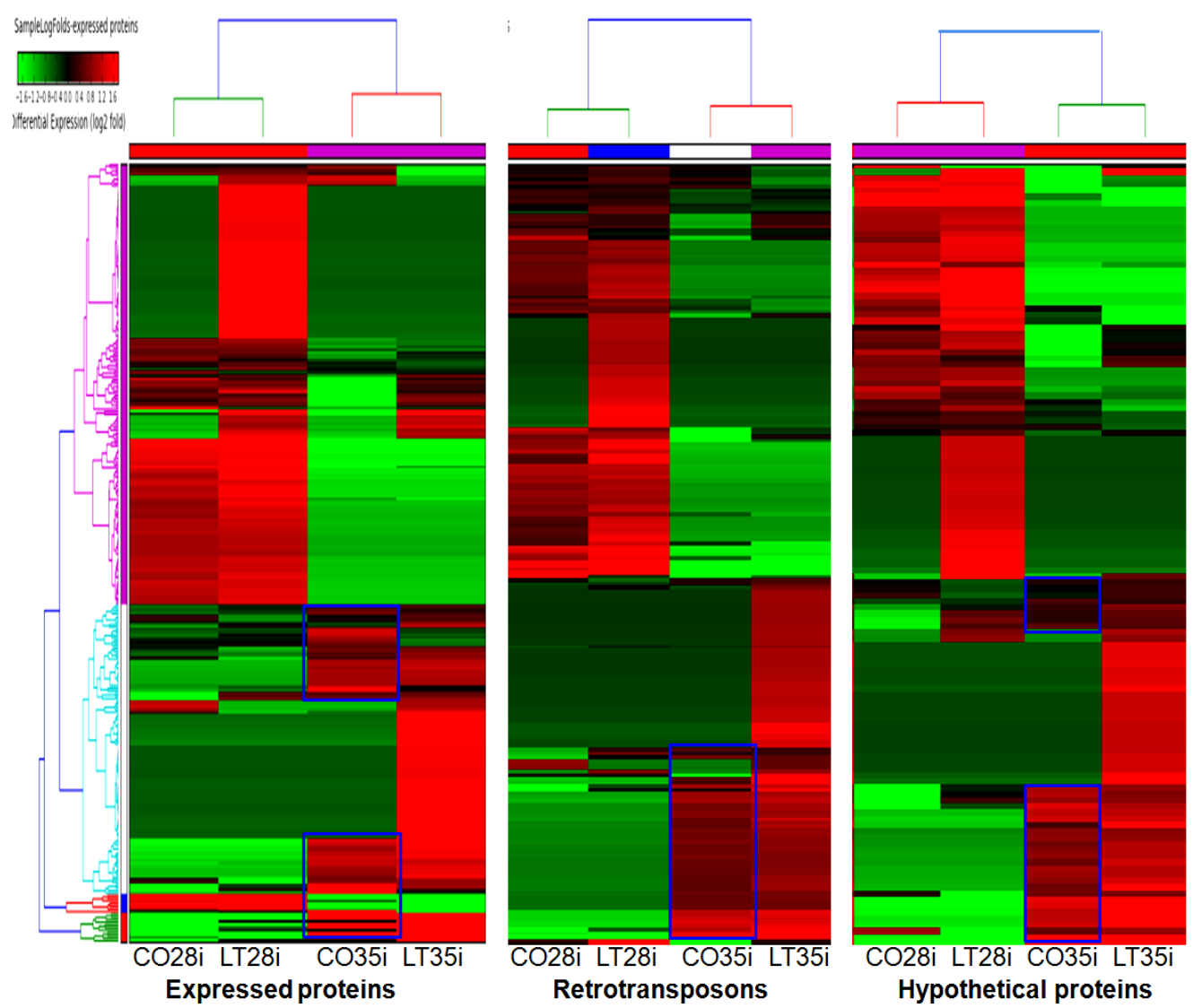

Figure 2.15: Heat map view of differentially expressed genes encoding for expressed proteins, retrotransposons and hypothetical proteins. Genes highlighted with a blue rectangle may be positive regulators of plant resistance at high temperature. CO28i, LT28i, CO35i and LT35i represent CO and LT plants infected with $M$. oryzae at $28^{\circ} \mathrm{C}$ and $35^{\circ} \mathrm{C}$, respectively. Red indicates higher expression while green represents lower expression. 
Chapter 3: Transcript profiles of Magnaporthe oryzae in planta expressed genes and rice defence response genes in plants pre-exposed to high temperature

Chapter 3: Transcript profiles of Magnaporthe oryzae in planta expressed genes and rice defense response genes in plants pre-exposed to high temperature

This chapter has been accepted for publication with revisions in: Onaga, G., Wydra, K.., Koopmann, B., Séré, Y. and von Tiedemann, A. (2016) Elevated temperature increases in planta expression levels of virulence related genes in Magnaporthe oryzae and compromises resistance in Nipponbare

First, third and last authors: Division of Plant Pathology and Crop Protection, Department of Crop Sciences, Georg-August-University Göttingen, Germany; second author: Georg-August-University Göttingen, CLBTropical and Subtropical Agriculture and Forestry, Germany; fourth author: Africa Rice Center, P.O Box 33581, Dar es Salaam, Tanzania.

\section{Introduction}

Temperature is arguably the most important environmental factor that influences plant pathogen interaction (Gregory et al., 2009). Temperature changes have the potential to alter the incidence and severity of plant disease epidemics and disease pressures, as well as to reshape the co-evolutionary relationships between plants and pathogens (Garrett et al., 2006). With the recent manifesting effects of climate change, it is expected that warmer temperatures will affect the biological processes leading to pathogenicity in most plant pathogens. This has been evident in some pathosystems; for instance, in wheat, studies have demostrated that higher mean temperatures correlated with hightened susceptibility to Cochliobolus sativus (Sharma et al., 2007). Although the optimal temperature range of $12-25^{\circ} \mathrm{C}$ used for wheat is considerably lower compared with an optimal temperature range of $28-32^{\circ} \mathrm{C}$ for rice, tropical and sub-tropical species have a narrow temperature range for growth and are likely to be more sensitive to changes in temperature (Ghini et al., 2011a). Thus, shifts in temperature may favor infection by some hemibiotrophic and necrotrophic pathogens.

Magnaporthe oryzae is conceivably the most important pathogen in global rice production. Annual rice harvest losses attributed to this disease range from $10-30 \%$, which is enough to feed 60 million people (Dean et al., 2012). The pathogenesis of M. oryzae is complex and a myriad of factors associated with virulence have been implicated. Several signal transduction events have been reported to modulate appressorium formation, penetration, and infectious growth (Wilson and Talbot, 2009). Even though the current knowledge of M. oryzae pathogenesis is extremely insightful, the role of environment on the pathogenesis of this pathogen is incomplete. Plants pathogen interaction is not a static phenotype, but is shaped by 
broad aspects of host and pathogen physiology, which are shaped by both genetic and environmental variations that often interact in nonlinear ways. Indeed, previous work has demonstrated that the influence of temperature on both pathogen fitness and host resistance affects blast pathogen infection, latency, lesion growth and sporulation, and has a significant role in blast epidemics (Ou, 1985; Teng, 1994). Hashioka (1950) reported an increase in the number of days required for rice blast infection when temperature is increased from 19 to $32^{\circ} \mathrm{C}$. In a study conducted on lesion development as a function of temperature, Kato and Kozaka (1974) showed that high alternating temperatures of $32 / 25^{\circ} \mathrm{C}$ and $32 / 20{ }^{\circ} \mathrm{C}$ resulted in initial rapid lesion growth, with a leveling off after 15 days. Moreover, warmer temperatures have been reported to induce severer blast epidemics in the cool subtropics than in the warm, humid subtropical regions, such as southern China, the Philippines and Thailand (Luo et al., 1998). Thus, temperature may shape the host phenotype and pathogen growth by either directly affecting pathogen growth or indirectly by its effect on host immunity. The pattern in which these temperature effects occur is not well understood. Obviously, rice blast like any other pathogen may alter primary and secondary metabolism, virulence factor production and infection efficiency when the normal growth temperature is altered (Ullrich et al., 1995; Budde and Ullrich, 2000). However, the success of infection will depend on the level at which the elevated temperature modulates both components of the pathosystem.

Here, we analyzed the effect of elevated temperature on the transcriptomes of rice and $M$. oryzae simultaneously using RNAseq. We used Nipponbare, the cultivar with available sequenced genome, to determine whether the infection process of $M$. oryzae is temperaturecontrolled. A comprehensive search for temperature regulated genes in $M$. oryzae and Nipponbare was carried out to gain fundamental insights into the major molecular adjustments in the pathogen and the host during $M$. oryzae infection at elevated temperature.

\section{Results}

\section{Effect of temperature on in planta fungal growth}

We investigated the interaction between rice and M. oryzae under normal growth temperature of $28^{\circ} \mathrm{C}$ and a warmer temperature of $35^{\circ} \mathrm{C}$ using Nipponbare rice cultivar as a host. Plants pre-exposed to $35^{\circ} \mathrm{C}$ had a severe disease phenotype, and displayed lesions within 4 days post inoculation (dpi). Alternatively, disease development was slow and mild in plants grown and inoculated at $28^{\circ} \mathrm{C}$ (Figure 3.1a). We used a quantitative real-time polymerase chain reaction 
(qPCR) to quantify M. oryzae biomass in infected leaf tissues of Nipponbare using Pot2 primers, $p f h 2 a$ and $p f h 2 b, 48$ hours after inoculation of plants exposed to $35^{\circ} \mathrm{C}$ and $28^{\circ} \mathrm{C}$. In accordance with the disease severity observed on infected leaves, the relative fungal biomass, as indicated by the DNA quantity of Pot2, was significantly higher in Nip35i than Nip28i (Figure 3.1b).

\section{RNAseq data analysis}

We analyzed the transcriptomes of Nipponbare and $M$. oryzae exposed to $35^{\circ} \mathrm{C}$ and $28^{\circ} \mathrm{C}$ simultaneously using RNA-seq. Two biological replicates of each treatment were sampled at $48 \mathrm{hpi}$ and sequenced (see materials and methods). We obtained an average of 57,550,449 and $58,189,196$ mixed transcriptome and the reference fungal reads, respectively, after raw reads filtering. Reads were aligned to Oryza sativa (ENSEMBL) and Magnaporthe oryzae 70-15 (Broad institute) genomes using Bowtie2 v2.0.2. More than 90\% $(53,843,253)$ of the mixed transcriptome reads mapped to Nipponbare genome. Reads that did not map onto the Nipponbare genome were mapped to the reference genome of isolate 70-15, and SAM files were generated using samtools 0.1.18. To improve on the genome coverage, the fungal reads that could not be aligned to $70-15$ were recombined with the mapped fungal read SAM files to guide assembly, and later processed with the de-novo assembler and re-mapped using CLC Genomics Workbench with default parameters i.e. two mismatches, minimum length fraction 0.9 , minimum similarity fraction 0.8 and maximum hits for a read of 10 . We obtained an average of $1.83 \%(1,053,057)$ reads that mapped to the fungal genome. To assess biological variation between replicates, we compared the read clustering pattern in all biological samples. Our experiments showed high levels of correlation for biological replicates (Pearson's Correlation Coefficient >0.90; Figure 3.2).

\section{Transcript profiles of $M$. oryzae and rice during infection at 28 and $35^{\circ} \mathrm{C}$}

Differentially expressed genes (DEGs) in Nip28i, Nip35i and Nip35c were grouped, based on reads per kilobase of exon model per million mapped reads (RPKM) values. We used a minimum of four-fold expression based on RPKM ratio to represent significant differentially expressed genes (DEGs), for either up or down regulation. From the significant fungal DEGs, 556 were exclusive to Nip35i, 519 were found only in Nip28i, and 4302 were detected in both treatments (Figure 3.3a; additional file S1). From the up-regulated fungal DEGs, 549 and 443 were identified as Nip35i and Nip28i-specific, respectively, and 1620 were up regulated in 
both treatments. We detected 143 and 212 down regulated DEGs as Nip35i and Nip28ispecific, respectively, and 2542 were down regulated in both treatments. To determine the fungal DEGs which differed significantly between Nip35i and Nip28i, the SAM files were analysed using SeqSolve software and Fisher's exact test was used test the level of significance between genes expressed in Nip35i and Nip28i. Direct visualization of significance and magnitude of differences in gene expression between Nip35i and Nip28i is presented using a volcano plot (Figure 3.3b). On the other hand, the rice response to pathogen infection and high temperature also showed differences among Nip28i, Nip35i and Nip35c treatments. A larger number of plants DEG (6458) were obtained in the comparison of Nip35i vs Nip28c, compared to 6013 in Nip28i vs Nip28c (Additional file S2). Among the differentially expressed rice genes responding to only high temperature (Nip35c vs Nip28c), 561 were up regulated and 763 were down regulated. From the anaylsis of DEGs between high temperature and $M$. oryzae infection, 1625 genes were exclusive to Nip35i, 1329 were found only in Nip28i, 420 were specific to Nip35c, whereas 652 were common to the three treatments (Figure 3.3c). The rice genes described throughout the text are provided in additional file $\mathrm{S} 2$.

\section{Transcriptional changes in Magnaporthe oryzae}

\section{GO annotations of fungal in planta induced genes}

Induced DEGs with at least 5 unique reads and more than 4 fold change expression in Nip35i and Nip28i, based on RPKM ratios, were selected for Gene Ontology (GO), Clusters of orthologous genes (COG) and Kyoto Encyclopedia of Genes and Genomes (KEGG) pathway representation using Fisher's Exact Test in DAVID database (Huang da et al., 2009b). The GO terms were categorized based on enrichment in biological processes (BP), cellular component (CC) and molecular function (MF). In the BP category, highly enriched subcategories in Nip35i were growth or development of symbiont on or near the host, regulation of transcription, carbohydrate transport, regulation of RNA metabolic process, oxidation reduction, ribosomal biogenesis and response to cAMP (Figure 3.4). In the CC domain, most of the up regulated DEGs were deemed intrinsic and integral to the cell membrane. The predominant annotations in the MF domain were transition metal ion binding, transcription regulator activity, electron carrier activity and peptidase activity. Terms related to interaction with host via secreted substance and secondary metabolic processes were more enriched in Nip28i than Nip35i. Terms related to transcription regulator activity, transition 
metal ion binding, peptidase activity and ATPase activity were highly enriched in Nip35i than Nip28i. Fatty acid metabolism and inorganic ion transport and metabolism COG group were the most highly enriched among COGs at both temperatures. Cell division and chromosome partitioning was specific to Nip35i, whereas carbohydrate transport and metabolism was highly enriched in Nip28i. The enrichment in the KEGG pathways was also influenced by temperature. In Nip35i, genes involved in N-glycan biosynthesis, meiosis and cell cycle were dominant, whereas valine, leucine and isoleucine degradation and starch and sucrose metabolism were dominant in Nip28i. Fatty acid metabolism was enriched in both treatments.

\section{High temperature influence on Known pathogenicity genes}

To predict $M$. oryzae pathogenicity genes affected by temperature, we performed BLASTp analysis of fungal DEGs against the pathogen-host interaction gene database (PHI database). Forty-four induced genes encoding for pathogenicity proteins were represented in both Nip35i and Nip28i, whereas 18 and 8 were identified as Nip35i and Nip28i-specific, respectively (Table S1). Among the 44 genes detected, MAS3 (MGG_12337), ribonuclease T2 (MGG_10510), PWL1, PWL2, Avr-Pita1, MoHox7, CUT1 (MGG_09100) and GAS2 (MGG_04202) were >60-fold up regulated at both temperatures but with a higher induction in Nip35i than Nip28i. Isocitrate lyase (ICL), calcium-transporting ATPase 3, MAC1, MoRgs5 (MGG_08735) and ABC proteins also showed increased abundance in Nip35i than in Nip28i. Moreover, Moatg11, HDL1, MoSFl1 and ERG2 were specifically induced in Nip35i. In contrast, endo-1,4-beta-xylanases (MGG_09098) and endoglucanase II (MGG_04547) were more induced in Nip28i than Nip35i. In addition, endo-1,4-beta-xylanases (XYL2; MGG_01542 and MGG_14243) and MoRgs6 were >70-fold induced in Nip28i but not in Nip35i. All genes previously reported to mediate docking and fusion of vesicles with the plasma membrane target site (Giraldo et al., 2013), were not significantly expressed, except polarisome component (Spa2; MGG_03703), which was induced 4-fold in Nip35i but not in Nip28i.

\section{Analysis of $M$. oryzae secretome during infection at high temperature}

About 1306 genes coding for putative secreted proteins have been predicted from M. oryzae genome (Zhang and $\mathrm{Xu}, 2014)$. However, the functional analysis of most M. oryzae secreted proteins is a pending challenge. In this study, we predicted transcripts categorized as signal peptides in the secretome of $M$. oryzae by analyzing a set of translated gene sequences using 
SignalP4.1 (Bendtsen et al., 2004), which detects the presence of a signal peptide in the amino terminus of a protein. We detected 367 and 342 signal peptides in Nip28i and Nip35i, respectively (Additional file S3). These signal peptides were analyzed using Phobius (Kall et al., 2007) and TMHMM (Krogh et al., 2001) to identify and remove proteins with TM helices, whereas proteins with mitochondrial signal peptides were identified using TargetP. Thereafter, a total of 189 induced genes encoding secreted proteins were detected, 36 of which were exclusive to Nip28i, 23 were specific to Nip35i, and 130 were identified in both treatments (additional file S4).

To gain insight into the functional categories of secreted proteins, their protein sequences were analyzed in order to identify PFAM domain categories associated with each induced gene (Finn et al., 2010). InterPro Scan was then used to confirm the distribution of recognizable protein domains among signal peptide sequences. The most frequently represented protein domains were glycosyl hydrolases, domains of unknown function (DUFs) and peptidases (Figure 3.5). To identify RxLR motifs that are thought to be required for host translocation of effectors, amino acid sequences of the secreted proteins were subjected to motif analysis using FuzzPro, a tool from EMBOSS package (Rice et al., 2000). A stringent analysis, which predicts RxLR patterns between initial 30-60 amino acids and appearing after the observed signal cleavage site (30 amino acids from the start), did not indicate any evidence of RXLR sequence presence in $M$. oryzae signal peptides. A less stringent approach, where any protein with RxLR motif and signal peptide cleavage site, without TM domain, identified 11 proteins with RxLR motifs in both Nip35i and Nip28i (Table 1), most of which were cell wall degrading glycosyl hydrolases. Interestingly, we detected a conserved hypothetical protein (MGG_14055) in this category, which was strongly induced in Nip35i

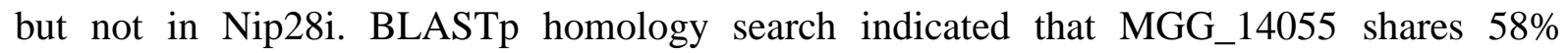
homology with $\beta$-xylosidase from Leptosphaeria maculans and $57 \%$ with Bipolaris sorokiniana. It also showed 56\% identity to glycosyl hydrolase 39 of Trichoderma atroviride, Bipolaris zeicola, Bipolaris oryzae and Bipolaris maydis. Consistent with this, Pfam domain searches indicated that MGG_14055 encodes a protein with glycosyl hydrolase 39 domain. We conducted multiple sequence alignment on all the putative secreted proteins using Phylogeny.fr platform (Dereeper et al., 2008). Three major clusters were formed with branch support values $>50$ (Figure 3.6). 
Chapter 3: Transcript profiles of Magnaporthe oryzae in planta expressed genes and rice defence response genes in plants pre-exposed to high temperature

\section{Expression of peptidases}

Sequences of predicted secreted proteins were subjected to MEROPS Batch Blast analysis (Rawlings et al., 2010) to identify potential peptidases expressed during infection at high temperature. We detected 15 and 14 up-regulated peptidase encoding genes in Nip35i and Nip28i, respectively (Table 2). Among the prominent genes were MGG_00230, carboxypeptidase S1 (MGG_03995), GAS2/MAS3 (MGG_04202) and epoxide hydrolase 2 (MGG_09603). Lipase 1, categorized as peptidase family S9 was also detected in both Nip35i and Nip28i. On the other hand, tripeptidyl-peptidase 1 (MGG_07404), phosphatidylethanolamine-binding protein (MGG_06844) and lipase 2 (MGG_02130) were strongly induced in Nip35i but not in Nip28i, whereas endoprotease endo-Pro-Aspergillus niger (MGG_02275) was specific to Nip28i.

Table 1: Predicted genes of M. oryzae with RxLR in their protein sequences

\begin{tabular}{|c|c|c|c|c|}
\hline \multirow[t]{2}{*}{ Feature ID } & \multirow[t]{2}{*}{ Description } & \multicolumn{2}{|c|}{$\log 2 \mathrm{FC}$} & \\
\hline & & Nip35i & Nip28i & \\
\hline MGG_14055 & conserved hypothetical protein & 10.69 & - & SignalP-noTM \\
\hline MGG_01885 & beta-glucosidase 2 & 6.27 & 4.48 & SignalP-noTM \\
\hline MGG_03257 & plasma alpha-L-fucosidase & 4.93 & 3.65 & SignalP-noTM \\
\hline MGG_11210 & beta-glucosidase 1 & 4.09 & 2.30 & SignalP-noTM \\
\hline MGG_10414 & cholinesterase & 4.07 & 3.37 & SignalP-noTM \\
\hline MGG_08088 & conserved hypothetical protein & 3.46 & 2.71 & SignalP-noTM \\
\hline MGG_09246 & oryzin & 3.46 & 3.14 & SignalP-noTM \\
\hline MGG_09732 & feruloyl esterase & 2.79 & 2.90 & SignalP-noTM \\
\hline MGG_03761 & conserved hypothetical protein & 2.29 & - & SignalP-noTM \\
\hline MGG_04078 & WSC domain-containing protein & 2.25 & - & SignalP-noTM \\
\hline MGG_00202 & conserved hypothetical protein & 2.12 & - & SignalP-noTM \\
\hline MGG_00042 & alpha-L-fucosidase precursor & - & 3.27 & SignalP-noTM \\
\hline MGG_01396 & periplasmic beta-glucosidase & - & 2.80 & SignalP-noTM \\
\hline MGG_01876 & chitinase 3 & - & 6.18 & SignalP-noTM \\
\hline MGG_08487 & cellobiose dehydrogenase & - & 3,52 & SignalP-noTM \\
\hline
\end{tabular}

\section{Increased expression levels of previously identified and novel secreted proteins of $M$.} oryzae at $35^{\circ} \mathrm{C}$

Magnaporthe oryzae virulence is highly variable and has been associated with diverse enzyme activities, including glycosyl hydrolases, peptidases and lipases among others. Among the genes previously reported, four biotrophy-associated secreted proteins (BAS1-4) identified by Mosquera et al. (2009) were induced >100-fold in both Nip35i and Nip28i, but with a higher expression level in Nip35i than Nip28i. 
Chapter 3: Transcript profiles of Magnaporthe oryzae in planta expressed genes and rice defence response genes in plants pre-exposed to high temperature

Table 2 Peptidase like genes of $M$. oryzae induced during infection at $35^{\circ} \mathrm{C}$ and $28^{\circ} \mathrm{C}$

\begin{tabular}{llcc}
\hline Feature ID & Description & $\begin{array}{c}\text { Nip35i } \\
\text { Log2FC }\end{array}$ & $\begin{array}{c}\text { Nip28i } \\
\text { Log2FC }\end{array}$ \\
\hline MGG_00230 & 98\% AVR-Pita2 & 13.20 & 13.09 \\
MGG_08416 lipase 1 & 10.88 & 11.50 \\
MGG_03995 & carboxypeptidase S1 & 10.50 & 9.02 \\
MGG_09603 & epoxide hydrolase 2 & 1030 & 10.50 \\
MGG_07844 & maltose permease & 9.88 & 8.18 \\
MGG_07584 & conserved hypothetical protein & 9.62 & 9.92 \\
MGG_04202 & MAS3 protein & 9.50 & 10.63 \\
MGG_02201 & endothiapepsin & 9.26 & 7.45 \\
MGG_03468 & intracellular hyphae protein 1 & 8.43 & 7.48 \\
MGG_07404 & tripeptidyl-peptidase 1 & 8.18 & 0.00 \\
MGG_06844 & conserved hypothetical protein & 7.88 & 0.00 \\
MGG_02130 & lipase 2 & 7.56 & 0.00 \\
MGG_01885 & beta-glucosidase 2 & 6.27 & 0.00 \\
& alpha/beta hydrolase fold-3 domain-containing & & \\
MGG_01369 & protein & 4.40 & 0.00 \\
MGG_04618 & amidophosphoribosyltransferase & 2.58 & 0.00 \\
MGG_02275 & endoprotease Endo-Pro-Aspergillus niger & 0.00 & 7.65 \\
MGG_09716 & carboxypeptidase A2 precursor & 0.00 & 4.52 \\
MGG_10744 & beta-lactamase family protein & 0.00 & 3.22 \\
MGG_02987 & para-nitrobenzyl esterase & 0.00 & 2.92 \\
MGG_07789 & 3-phytase & 0.00 & 2.63 \\
\hline
\end{tabular}

Among the genes previously published by Kawahara et al. (2012) as candidate novel fungal effectors, MGG_09379, MGG_10705, MGG_10004, MGG_12655, MGG_10080, MGG_09628, MGG_09629 and MGG_08715 were strongly induced in both Nip35i and Nip28i. In addition, two putative secreted proteins with LysM domains, annotated as intracellular hyphae protein 1 (MGG_03468 and Slp1; MGG_10097) were >30-fold induced in both Nip35i and Nip28i. MGG_10097 was not expressed in mycilia and appears to be exclusively expressed in planta. In addition, a conserved hypothetical protein (MGG_02405) also with LysM domain was induced >3-fold in Nip35i than Nip28i. A histidine phosphatase superfamily protein encoding gene (3-phytase A; MGG_15390) was also induced >100-fold in both Nip35i and Nip28i, but with a higher induction in Nip35i. Alternatively, several genes showed specificity in expression. For instance, MGG_11009, a putative cell wall galactomanno (GM) protein, which shares $99 \%$ sequence identity with hydrophobic surface binding protein A (HsbA) was $>100$-fold induced in Nip35i but not in Nip28i. Additional noteworthy genes with a >30-fold induction in Nip35i compared to Nip28i were MGG_13116, Cupredoxin (MGG_08291), W rich C domain containing protein (MGG_04263) and polygalacturonase A (MGG_08938). 


\section{Induction of genes containing partial sequences of Avr genes}

A total of 7 Avr genes have been cloned in M. oryzae, including AvrPi-ta, Avr1-CO39, ACE1, AvrPiz-t, AvrPii, AvrPia, and AvrPik/km/kp (Li et al., 2012). We performed database similarity searches using the several predicted and hypothetical proteins present in our dataset to identify sequences belonging to Avr genes. Among the strongly induced genes, AVR-Pita1 (MGG_15370), MGG_00230 and MGG_16175 with 98\% and 95\% sequence identity to AVR-Pita2, respectively, MGG_16585 and MGG_18041 with 99\% and 100\% sequence identity to Avrpiz-t were induced >100-fold in Nip35i compared to Nip28i. In addition, the gene MGG_15972 with 99\% identity to AvrPik/kp/km was $>100$-fold induced in Nip35i compared to Nip28i. Some genes shared partial sequence identity to known Avr genes and transposable elements. For example, MGG_16585 shared 99\% partial sequence identity with both Avrpiz-t and AVR-Pita1 whereas MGG_15972 was 99\% identical to AvrPik/kp/km, AvrPi7, flanked by $3 \%$ coverage of $98 \%$ sequence from Pot2. MGG_16570 shared 92\% sequence homologywith Avrpiz-t and AVR-Pita1 and was induced exclusively in planta. The presence of genes with Avr identical sequences interrupted with transposable elements is consistent with the previous report (Li et al., 2012). Disease resistance response is regulated by plant resistance $(\mathrm{R})$ genes whose products directly or indirectly recognize products of the corresponding pathogen avirulence (Avr) genes. To find out whether the presence of these putative Avr transcripts corresponded with the $\mathrm{R}$ genes currently used as differentials, we tested 32 differential lines against TAN211.16. Interestingly, Piz- $t$ was highly resistant at both $28^{\circ} \mathrm{C}$ and $35^{\circ} \mathrm{C}$ (Figure 3.7). The other lines with increased resistance included Pita-2, Pii and Pi9.

\section{Effect of high temperature stress on lipid metabolism of $M$. oryzae during infection}

M. oryzae mobilizes lipid bodies for the synthesis of glycerol through lipolysis, especially during appressorium formation (Wilson and Talbot, 2009). M. oryzae possesses 19 genes that encode intracellular lipases, and that degrade and utilize intracellular lipids, most of which have not been functionally characterized. We detected a high induction of lipase 1 (MGG_08416), with >100-fold higher expression in Nip28i than in Nip35i (additional file S1). Transcription of other lipases, including lipase 4 (MGG_00314) and MGG_09839 was detected in both Nip28i and Nip35i. However, secreted lipase family protein (MGG_04257) was $>40$-fold higher induced in Nip35i than in Nip28i. Among the genes involved in the synthesis of the cell membrane components, non-specific lipid-transfer protein 
(MGG_02409), 7-dehydrocholesterol reductase (MGG_03765) that catalyzes the last step of cholesterol biosynthesis, fatty acid-binding protein (MGG_07337) and fatty acid synthase Sacetyltransferase (MGG_04775) that catalyzes fatty acid synthesis intermediates from acetylCoA were $>15$-fold up-regulated in both Nip35i and Nip28i. In addition, a putative lipid phosphate phosphatase 1 (LPP; MGG_14955) was strongly induced in both Nip35i and Nip28i, but with a higher induction in Nip35i. In contrast, glycerol-3-phosphate acyltransferase (MGG_01894) required for phospholipid biosynthesis and mannosylinositol phosphorylceramide (MIPC) synthase (MGG_13744) that catalyzes the synthesis of sphingolipids were specifically induced in Nip35i, whereas a conserved hypothetical protein (MGG_15479) encoding for esterase, SGNH hydrolase-type domain containing protein was induced 55-fold in Nip28i but down-regulated in Nip35i.

Very long chain fatty acids have been reported to accumulate in fungal cells with a disrupted acyl-CoA synthetase, FAT1 (Oh et al., 2008). We detected 21-fold and 11-fold induction of long chain fatty acyl-CoA synthetase (MGG_08257) in Nip35i and Nip28i, respectively, suggesting higher fatty acid catabolism during infection at $35^{\circ} \mathrm{C}$ than at $28^{\circ} \mathrm{C}$. Indeed several genes involved in the initial step of fatty acid $\beta$-oxidation, including acyl-CoA dehydrogenase (MFP1; MGG_03418), which encodes a multifunctional $\beta$-oxidation protein, isocitrate lyase (MGG_04895) and malate synthase (MGG_02813) of the glyoxylate cycle, peroxisomal acetyl carnitine transferase (PTH2; MGG_01721) and MgPex6 (MGG_00529) required for peroxisome biogenesis were more induced in Nip35i than Nip28i. In contrast, mitochondrial $\beta$-oxidation protein enoyl-CoA hydratase (MGG_08775) required for neutralizing host defenses at the post-penetration stage was induced in both Nip35i and Nip28i.

\section{Effect of high temperature stress on carbohydrate metabolism of $M$. oryzae during infection}

Growth of $M$. oryzae in the plant host involves utilization of carbohydrate carbon sources, including glucose and glycerol. Storage carbohydrates include glycogen and polyols, whereas chitin, glucans and xylans are primary constituents of the fungal cell wall. We searched for carbohydrate metabolism encoding genes using the CAZy annotation pipeline (http://www.cazy.org), and compared the genes presented in $M$. oryzae inventory to the genes expressed in our study. We detected 216 genes in our dataset, 174 of which were common to both Nip35i and Nip28i (Additional file S5). Glycosyl hydrolases (GHs), glycosyltransferases 
(GTs) and carbohydrate esterases (CEs) were the most abundant in our dataset. Among the GHs, MAS3 featured as the most prominent in this category. Three GH43 genes (MGG_09098, MGG_00570 and MGG_00574) also exhibited high induction during infection at both temperatures. Among the CEs, ferulic acid esterase A (MGG_10040), acetylxylan esterase 2 (MGG_15403) and fungal cellulose binding domain-containing protein (MGG_01403) were induced >50-fold at both temperatures.

Chitin is a microfibrillar b-1,4-linked homopolymer of $\mathrm{N}$-acetylglucosamine (GlcNAc) synthesized by polymerase activity of chitin synthases. A putative chitin deacetylase (MGG_09159), which catalyzes the conversion of chitin to chitosan was up-regulated >60fold in both Nip28i and Nip35i. In contrast, two chitinases (acidic mammalian chitinase; MGG_04732 and chitinase 1; MGG_05533) were more induced in Nip35i than Nip28i, whereas a hypothetical protein (MGG_05351) with a chitin-binding, type 1 domain was $>100$-fold up-regulated in Nip28i than Nip35i. Among the genes that exhibited specific expression, GH3 genes (e.g., MGG_08985 and MGG_01885), were specific to Nip35i, whereas GH7 exoglucanase 1 (MGG_10712) and GH10 endo-1,4-beta-xylanase (Xy12; MGG_01542) were strongly induced in Nip28i. Genes encoding for pectin degrading enzyme (e.g., PL1; MGG_07566) were more induced in Nip28i, although PL4 (MGG_06041) was more induced in Nip35i than Nip28i. The expression of other genes involved in the utilization of non-preferred carbon sources, including genes encoding for alcohol dehydrogenase 1, galactose oxidase, levanase, NADP-dependent alcohol dehydrogenase 6, and a hypothetical protein (MGG_10051) which shares 72\% sequence identity with cellobiose dehydrogenase, also showed significant up regulation (>70-fold) in both Nip35i and Nip28i. In addition, mannosyl-oligosaccharide 1,2-alpha-mannosidase IC (MGG_00994) and cytochrome P450 for alkane assimilation (MGG_05908) were also strongly induced at both temperatures. In contrast, a gene involved in sugar alcohol degradation (alcohol oxidase; MGG_09072) was $>100$-fold induced in Nip35i than Nip28i. Also present were genes involved in building fungal glycoproteins and other cell wall components, including mannosyl-oligosaccharide 1,2-alpha-mannosidase IB, chitobiosyldiphosphodolichol beta-mannosyltransferase, dolichyldi-phosphooligosaccharide-protein glycotransferase and dolichyl pyrophosphate Glc1Man9GlcNAc2 alpha-1,3-glucosyltransferase. These genes were highly induced in Nip35i than Nip28i. 


\section{Stress and secondary metabolism induced genes during infection at high temperature}

Genes involved in stress and defence responses have been shown to be differentially expressed during infection in M. oryzae (Rauyaree et al., 2004). In this study, highly up regulated genes in relation to high temperature stress included DnaJ domain-containing proteins (MGG_14699; 33-fold), chaperone protein dnaJ 2 (MGG_07502; 8-fold), heat shock factor protein HSF24 (MGG_03242; 6-fold), HSP40 (SCJ1; MGG_07502, 8-fold), HSP70 domain containing protein (MGG_09631; 24-fold) and methyltransferase (MGG_05053; 67fold). In addition, a putative DnaJ domain-containing protein (MGG_03581), a flocculation suppression protein (MGG_06971), predicted protein (MGG_06486) and mitochondrial chaperone (MGG_14588) were more induced in Nip35 than Nip28i. SCJ1 was previously reported to be induced together with LHS1 during vegetative growth and conidiation (Yi et al., 2009), suggesting its involvement in M. oryzae pathogenicity. We used the cytochrome P450 database (FCPD) pipeline (http://p450.riceblast.snu.ac.kr/) to identify cytochrome P450 encoding genes. Prominent genes identified in this category included averantin oxidoreductase (MGG_10070), pisatin demethylase (MGG_05235), metalloproteinase (MGG_15370) and epoxide hydrolases (e.g., MGG_03070 and MGG_09603). Additional noteworthy genes were ent-kaurene oxidases (e.g., MGG_02294), CYP589A1 (MGG_12497), polyketide synthase (MGG_00241), citrinin polyketide synthase (MGG_10011) and CYP611A1 (MGG_10355) (Additional file S6). In contrast, two cytochrome P450s (CYP563A1; MGG_07593 and CYP608A1; MGG_04337) were induced in Nip35i but not in Nip28i. Both genes putatively encode for cytochrome P-450 monoxygenases required for isotrichodermin C-15 hydroxylation in trichothecene biosynthesis. A high induction of pentalenene synthase (MGG_10671), phenylacetone monooxygenase (MGG_00824) and geranylgeranyl pyrophosphate synthetase (MGG_03432) was also detected in both Nip35i and Nip28i, suggesting that their function is independent of high temperature effect. Peroxidases have been suggested to be integral components of antioxidant defense system in M. Oryzae (Egan et al., 2007; Fernandez and Wilson, 2014b). We searched the Fungal Peroxidase Database (fPoxDB) for peroxidase encoding genes and found 27 putative peroxidases in $M$. oryzae genome. Among them, MoCCP2 (MGG_10368), MoVPX1 (MGG_02210) and CATA (MGG_10061) were more induced in Nip35i than Nip28i. In addition, thioredoxin peroxidase (MGG_07503), oxidoreductase (MGG_02115), L-ascorbate oxidase (MGG_14307) were stronger induced in Nip35i than Nip28i, suggesting an adaptive response to the ROS environment in plants, probably induced in response to high temerature. 
Alternatively, MoHPX2 was highly induced in Nip28i but was not significantly induced in Nip35i.

Genes encoding for 2-oxoglutarate-dependent ethylene/succinate-forming enzyme (MGG_10266) and 1-aminocyclopropane-1-carboxylate deaminase (MGG_02812) were also higher induced in Nip35i than in Nip28i. In addition, salicylate hydroxylases (e.g., MGG_03764 and MGG_10012) and 2,3-dihydroxybenzoic acid decarboxylase (MGG_03793) required for SA degradation and conversion of 2,3-dihydroxybenzoic acid to catechol, respectively, were induced $>100$-fold in both Nip35i and Nip28i. The gene encoding for general amino acid permease (GAP1; MGG_07606), previously shown to be downregulated in the study of Mathioni et al. (2012), was up and down-regulated in Nip35i and Nip28i, respectively.

\section{Magnaporthe oryzae induced genes encoding for transport proteins}

Fungal pathogenesis requires active translocation of various molecules, including water, amino acids, proteins, soluble carbohydrates, fatty acids, and enzymes, and removal of antifungal plant defense compounds. Our data revealed differential expression of genes encoding various transporters, including ATP-binding cassette (ABC) and major facilitator (MFS) transport proteins and ion transporters (Additional file S7). From the up regulated ABC transporters, multidrug resistance protein CDR1 (MGG_07848) was strongly induced in both Nip35i and Nip28i. The same was true for ABC-type $\mathrm{Fe}^{3+}$ transport system (MGG_10060). In contrast, ABC transporter CDR4 (ABC1; MGG_13624), previously reported to be strongly induced by azole fungicides and the rice phytoalexin sakuranetin $(\mathrm{Oh}$ et al., 2008) was induced in Nip35i but not in Nip28i. Among the major facilitator superfamily (MFS) genes, two genes (MGG_00144 and MGG_01877) were strongly induced (>100-fold) in Nip35i but not in Nip28i. Other MFS encoding genes (e.g., MGG_06794 and MGG_02167), which were previously reported to share homology with toxin efflux pumps and multidrug transporters (Oh et al., 2008), were >30-fold induced in Nip35i than Nip28i. We further found that malic acid transport protein (MGG_09085), inorganic phosphate transporter PHO84 (MGG_03299), ammonium transporter 1 (MGG_04576), phosphate transporter (MGG_04251), urea active transporter (MGG_09063), aquaporins and oligopeptide transporter 2 (MGG_08984) were more induced in Nip35i than in Nip28i. Furthermore, the major myo-inositol transporter iolT (MGG_08776) and calcium-transporting 
ATPase 3 (MGG_10730) were induced >20-fold in Nip35i compared to Nip28i, which suggests that these genes play a role in plant infection at high temperature. In contrast, the MFS gene encoding for $\mathrm{H}(+) /$ hexose cotransporter 1 (HUP1; MGG_08617) involved in sugar transport was $>100$-fold induced in Nip28i compared to Nip35i. Other noteworthy genes upregulated at both temperatures included a quinate permease (MGG_07779), a lactose permease (MGG_00085), two maltose permeases (MAL31; MGG_07844 and MAL61; MGG_08266), peroxisomal 2,4-dienoyl-CoA reductase (SPS19; MGG_05138.6), an aquaporin-9 (AQY2; MGG_13615), glycerol kinase (GUT1 MGG_10005) and a sorbose reductase (SOU1; MGG_07883). The induction of these genes is consistent with the previous report (Mathioni et al., 2012).

\section{Regulation of transcription factors in $M$. oryzae during infection at high temperature}

A list of all $M$. oryzae transcription factors expressed in this study was identified using InterPro terms associated with DNA-binding motifs as described by Park et al. (2013). Using InterPro domain classification, we identified fungal-specific $\mathrm{Zn} 2 \mathrm{Cys} 6$ and $\mathrm{C} 2 \mathrm{H} 2$ zinc finger as dominant TF genes expressed during infection (Additional file S8). All M. oryzae TFs present in the Fungal Transcription Factor Database (FTFD, http://ftfd.snu.ac.kr) were also analyzed for domain categrization. From the 120 TFs identified as up regulated, 112 and 89 were expressed in Nip35i and Nip28i, respectively. A hypothetical protein with Homeodomain (MGG_09628) was induced >100-fold at both temperatures. Two C2H2 zinc finger (MGG_10280 and MGG_10276) and a winged helix repressor (MGG_08377) were specifically induced in planta. In contrast, a nucleic acid-binding, OB fold (MGG_04335) was induced >100-fold more in Nip35i than Nip28i, whereas for C2H2 zinc finger (MGG_15489) the opposite was true. In addition, two Zn2Cys6 genes (MGG_02879 and MGG_00049) and C2H2 zinc finger (MGG_04546) were >50-fold induced in Nip35i, but not in Nip28i. One $\mathrm{TF}$, previously reported to be a general regulator controlling multiple processes in $M$. oryzae, MGG_00021 (Park et al., 2013), was more induced in Nip28i than Nip35i. Conversely, the MST12 (MGG_12958) transcription factor that was previously reported to be regulated by PMK1 during penetration and invasive growth, and Ste50 (MGG_05199), an adaptor protein for the upstream components of the PMK1-MST7-MST11 cascade, were slightly induced (>4fold) in Nip35i, but not in Nip28i. Additional noteworthy genes that were highly induced in Nip35i than Nip28i were cutinase transcription factor 1 beta (MGG_08199), transcriptional activator xlnR (MGG_02880), and several genes of unknown function with Zn2Cys6 domain, 
including MGG_08777 and MGG_07368. Interestingly, two TF genes (MGG_00080 and MGG_10575) were strongly down-regulated at high temperature (>100-fold). The functional role of these genes remains ellusive.

\section{Temperature-responsive, promoter motifs identified for induced genes specific to Nip35i}

We analyzed the upstream intergenic sequences of genes with $>15$-fold up-regulation that were specifically expressed during infection at high temperature to identify motifs that correlate with temperature-dependent changes using the MEME algorithm. MEME analysis returned three motifs with E-value $<0.1$. Motif 1 was enriched with tyrosine kinase phosphorylation site between amino acids 33-40 and casein kinase II phosphorylation site 3437. Motif 2 consisted of protein kinase $\mathrm{C}$ phosphorylation site 32-34, N-myristoylation sites 23-28 and 28-33 and casein kinase II phosphorylation site 43-46. Motif 3 consists of Nglycosylation site 7-10 and N-myristoylation site 15-20. Motif 2 and Motif 3 were the most abundant in the upstream regions of genes showing significant enrichment (Figure 3.8).

\section{Rice plant reactions in response to $\mathrm{HT}$ and $M$. oryzae infection}

\section{GO enrichment and transcript abundance in rice during $M$. oryzae infection}

On the basis of GO categories, rice transcripts were assorted into several biological categories using AgriGO. Parametric analysis of gene set enrichment (PAGE) tool was used to identify GO terms significantly associated with Nip35c, Nip35i and Nip28i versus Nip28c comparisons. PAGE method is based on central limit theorem, and considers gene expression level using a two-tailed test to count $\mathrm{Z}$ scores. The up-regulated transcripts were grouped into 21 GO terms in infected samples (Nip28i and Nip35i) and 16 categories in Nip35c vs Nip28c (Figure 3.9). The prominent categories in all the treatments were related to metabolic process, followed by response to stimulus, response to stress and protein modification process. To identify common transcriptional changes due to temperature effect and $M$. oryzae infection, the gene expression profiles obtained from Nip35c and Nip35i were compared to Nip28i. Among the genes with $>40$-fold induction in both Nip35c and Nip35i, were genes encoding for peptidyl-prolyl isomerase (OsFKBP65; LOC_Os04g28420), photosystem II $10 \mathrm{kDa}$ polypeptide chloroplast precursor (LOC_Os07g05360), S-adenosyl-L-methionine benzoic acid/salicylic acid carboxyl methyltransferase (OsBISAMT1; LOC_Os11g15040) and expressed protein (LOC_Os09g13440), which is consistent with our previous findings. 


\section{Activated signaling pathways involved in rice defense against pathogen attack at high temperature}

Plant defense response genes are transcriptionally activated by pathogens depending on specific environmental conditions. In this study, we detected several genes involved in recognition (receptors), signaling, transcription modulation and downstream cascades associated with plant defense. We detected 204 receptor like kinases, among which SHR5 genes (e.g., LOC_Os08g10310) were induced >100-fold in both Nip35i and Nip28i. LOC_Os08g10310 was slightly induced (12-fold) in Nip35c, suggesting that SHR5 is relatively more responsive to $M$. oryzae infection than high temperature. Additional noteworthy genes were wall-associated receptor kinase-like 22 (LOC_Os10g07556), receptor protein kinase-like (LOC_Os10g05570), LOC_Os05g16930, OsWAK89a, OsWAK95, OsWAK88, OsWAK80, OsWAK71, OsWAK37, OsWAK38 and CRK5 (LOC_Os04g56430). LOC_Os05g16930 shares 85\% sequence identity with Oryza brachyantha LRR receptor-like serine/threonine-protein kinase, and was specifically induced in Nip35i.

In rice- $M$. oryzae interactions, MAPKs play significant roles in transducing defense signals to downstream targets. We detected induction of OsMPK13 (LOC_Os02g04230) and OsMPK5 in both Nip35i and Nip28i. Both genes have been shown to be induced by ACC and M. oryzae infection (Reyna and Yang, 2006), and are markers for negative plant resistance in rice. Also prominent were calcium response signaling genes, including OsCML25, OsCML26, calmodulin binding proteins (e.g., LOC_Os11g44700 and LOC_Os11g44630), which were up regulated $>50$-fold during $M$. oryzae infection. These genes were undetected in samples exposed to only high temperature, except OsCIPK4 (LOC_Os12g41090).

One important feature of plant response to stress is cell wall modification. We detected 501fold and 184-fold induction of a beta-expansin gene (LOC_Os06g47360) in Nip35i and Nip28i, respectively. Another expansin gene (LOC_Os09g29710) was 84-fold and 40-fold induced in Nip35i and Nip28i, respectively. Expansins have the ability to induce extension of cell walls and enhance stress relaxation (Valdivia et al., 2007). NCBI BLAST search comparisons of LOC_Os06g47360 revealed high sequence similarity with blight-associated protein p12, which was previously associated with citrus blight (Ceccardi et al., 1998). 
During plant pathogen interaction, pathogens that successfully elude the first layer of defense secrete effectors that deregulate PTI. To counteract this, plant resistance (R) proteins recognize effectors and activate effector-triggered immunity. Genes encoding for coiled-coil motif nucleotide binding site-leucine-rich repeat (CC-NBS-LRR) proteins (e.g., LOC_Os04g25900) and NBS-ARC RPM1 like encoding gene (LOC_Os08g16070) were upregulated >100-fold in Nip35i, but not in Nip28i. LOC_Os04g25900 was previously shown to be induced in rice challenged with Brown plant hopper (Kamolsukyunyong et al. 2013), and is apparently induced by ethylene.

The expression of genes encoding for ROS producing enzymes was evidently lower in response to both high temperature and infection. Alternatively, the induction of genes encoding for ROS scavenging enzymes, including peroxidases, cytochrome P450s, thioredoxins, catalase isozyme $\mathrm{B}$, glutaredoxin and glutathione $\mathrm{S}$-transferases was evident at both temperatures.

\section{Hormone signaling pathway in relation to high temperature in Nipponbare}

Phytohormones regulate plant resistance through mediation of signaling pathways in response to environmental stresses. The role of salicylic acid (SA), ethylene (ET) and jasmonic acid (JA)-mediated signaling in response to pathogen infection is well documented (Denancé et al., 2013). SA is also a key mediator of high temperature response in plants (Bita and Gerats, 2013). We previously reported that MeSA is emitted in response to both high temperature and M. oryzae infection (Onaga et al., unpublished), which is consistent with the results of this study. We also indicated that MeSA was not an inducer of a defense response, but probably invokes the JA pathway to boost rice defense against $M$. oryzae. Indeed, we detected $>50$-fold induction of 12-oxophytodienoate reductase (LOC_Os06g11210), LOXC2 (LOC_Os08g39850), AOS (LOC_Os03g12500), LOX2 (LOC_Os08g39840) and several other LOX and AOS, but we did not detect MeJA production related genes as previously found in our study involving $\mathrm{R}$ genes. We also detected two BRASSINOSTEROID INSENSITIVE 1 (BRI1)-associated receptor kinase 1 encoding genes (LOC_Os11g31540 and LOC_Os11g31530), which were >100-fold induced. BRI1s are members of the leucine-rich repeat RLK (LRR-RLK) family that perceive signals from brassinosteroids (BRs), a steroid hormone that regulates a wide range of developmental and physiological processes in plants (Tang et al., 2010). BRs induce the expression of 12-oxophytodienoate reductase and 1- 
aminocyclopropane-1-carboxylate synthase (Nakashita et al., 2003). This is consistent with a strong induction of ET associated genes, including 1-aminocyclopropane-1-carboxylate oxidase (LOC_Os08g30100; 405-fold), naringenin,2-oxoglutarate 3-dioxygenase (LOC_Os04g49194; 286-fold and LOC_Os04g49210; 172-fold), ACC (LOC_Os04g48850) and ERF1 (LOC-Os09g39850 and LOC_Os03g64260), and the JA biosynthesis pathway enzyme 12-oxophytodienoate reductase. Other noteworthy genes were flavonol synthase/flavanone 3-hydroxylase (DMR6), regulators of auxin signaling such as OsIAA25, OsGH3.2 and OsIAA13 and SMALL AUXIN-UP RNA genes (OsSAUR26, OsSAUR20, OsSAUR24, OsSAUR25 and OsSAUR2).

\section{Induced transcription factors in rice during M. oryzae infection at 35 and $28^{\circ} \mathrm{C}$}

Transcription factors (TFs) play an essential role in modulating defense, hormone and growth related genes. Superfamily TFs, including WRKYs, MYBs, bHLH and C2H2 were significantly expressed in this study. Among the WRKYs, OsWRKY84, WRKY47, WRKY49, WRKY107, WRKY19 were >50-fold induced in Nip35i. Six MYB genes, LOC_Os11g01480, LOC_Os12g01490, LOC_Os08g33150, LOC_Os09g23620, LOC_Os05g37060 and MYB4 (LOC_Os02g41510) showed >20-fold induction in both Nip35i and Nip28i. Induction of OsMYB4 is consistent with our previous study (Onaga et al., unpublished). We also detected up regulation of several pentatricopeptide PPR repeat containing proteins, including LOC_Os03g55840 and LOC_Os11g37330, which share structural identity with some of the MYB encoding genes. The induction of genes encoding for AP2 domain containing proteins (e.g., OsEREBP1; LOC_Os09g39850, LOC_Os03g08470 and LOC_Os03g08460) is consistent with our previous findings, and is apparently associated with the increase in the amount of the JA precursor 12-Oxophytodienoic acid (OPDA) in the ethylene-JA interplay against pathogen infection. Other up regulated TFs include ZOS8-14 - C2H2 zinc finger protein (LOC_Os08g44830; 97-fold), CCT/B-box zinc finger protein (LOC_Os02g49230; 92-fold) and core histone H2A (LOC_Os03g06670; 15-fold). Besides this, we detected >100-fold induction of putative aspartic proteinase nepenthesin encoding genes (LOC_Os10g39300, LOC_Os10g39260 and LOC_Os07g34850). These genes were induced in concert with defensin and thionin encoding genes, including DEFL9 (LOC_Os06g22919). 


\section{Genes involved in secondary metabolism in rice response to pathogen attack}

We detected several genes involved in secondary metabolism, some of which were documented in our previous study (Onaga et al., unpublished). Our data exhibited a significant induction (>100-fold) of hydroxycinnamoyl-CoA shikimate (HCT) encoding genes (e.g., LOC_Os04g56910, LOC_Os10g23310 and LOC-Os09g37200) and a putative mannitol dehydrogenase (LOC_Os09g23530). HCT is a key enzyme in the conversion of $p$-coumaroyl CoA to $p$-coumaroyl shikimic acid and caffeoyl CoA during lignin biosynthesis. Mannitol dehydrogenase is involved in the conversion of intermediates, including conferaldehyde to monilignols such as coniferyl alcohols. Also prominently expressed was LOC_Os08g34790, which encodes an AMP-binding domain-containing protein, a close homologue of 4coumaroyl-CoA synthase 2 (4CL) involved in the conversion of $p$-coumaric acid to $p$ coumaroyl CoA. A number of caffeoyl-CoA 3-O-methyltransferase putative encoding genes, including LOC_Os09g30360 and LOC_Os08g05790, were also up regulated. Interestingly, most PAL encoding genes (PAL2; LOC_Os02g41670, LOC_Os05g35290 and LOC_Os02g41680) were expressed, but kept lower in Nip35i than in Nip28i, suggesting that high temperature affected PAL gene expression, and this is consistent with our previous findings. Genes involved in phytoalexin biosynthesis, including putative Anthranilate Nhydroxycinnamoyl/benzoyltransferase 3 (LOC_Os08g01950), diterpene cyclase genes, OsKSL4 (LOC_Os04g10060), OsKSL7 (LOC_Os02g36140) and OsKSL8 (LOC_Os11g28530) were >20-fold induced during M. oryzae infection at both temperatures. The same was true for OsKSL10 (LOC_Os12g30824), OsCPS4 (LOC_Os04g09900) and OsCPS2 (LOC_Os02g36210). Other genes induced >20-fold included chalcone and stilbene synthases (LOC_Os07g34190 and LOC_Os07g34260). Cytochrome P450 genes involved in phytoalexin biosynthesis (Park et al., 2013), such as CYP99A2 (LOC_Os04g10160), CYP99A3 (LOC_Os04g09920), CYP76M8 (LOC_Os02g36070) and CYP71Z7 (LOC_Os02g36190), were induced >100-fold in response to M. oryzae infection but were not detected in plants exposed to only high temperature. The same was true for the sex determination protein tasselseed-2 (LOC_Os04g10010), a homolog of dehydrogenase gene involved in phytoalexin biosynthesis.

\section{Expression of pathogenesis related (PR) genes}

To identify PR genes specifically induced in Nip35c, Nip35i and Nip28i, we examined our data using known PR genes reported in literature. We detected higher induction of PR3 
(CHIT17; LOC_Os05g33130) and $\beta$-1,3-glucanase (Gns6; LOC_Os01g71350) in response to only high temperature. In addition, a cupin domain containing protein (OsGLP8-12; LOC_Os08g13440), thaumatin-like protein 1(PR5K; LOC_Os03g14050), PR10-b (LOC_Os12g36850), WIP3 (OsPR4c; LOC_Os11g37950) and WIP4 (OsPR4b; LOC_Os11g37960) were induced >5-fold in response to only high temperature. To determine the $\mathrm{PR}$ gene expression changes in response to both high temperatures and $M$. oryzae infection, the genes expressed in Nip35i were compared with genes induced in Nip28i. Of the 112 PR genes identified from literature, 42 were up regulated in Nip35i as compared to 39 in Nip28i. A similar number of genes $(n=6)$ were down regulated in both treatments. The genes LOC_Os10g11500 similar to PR1-a, PR2 (Gns5; LOC_Os01g71340), PR8 (Gns11; LOC_Os07g35480) were induced >50-fold in Nip35i but not in Nip28i. Transcript levels for the cupin domain containing proteins, including OsGLP8-10 (LOC_Os08g09060), OsGLP8-9 (LOC_Os08g09040), OsGLP8-8 (LOC_Os08g09020), OsGLP8-5 (LOC_Os08g08990) were $>100$-fold induced in Nip35i compared to Nip28i. In addition, the genes PR1 (LOC_Os07g03730), PR8 (Oschib2; LOC_Os10g28120) and PR2 (Gns10; LOC_Os01g51570) were also >100-fold induced in Nip35i than in Nip28i. On the other hand, OsGLP8-12 (LOC_Os08g13440) was >100-fold induced in Nip28i compared to Nip35i. Similarly, OsGLP8-3 (LOC_Os08g08970) was induced 137-fold in Nip28i but not in Nip35i.

\section{Confirmation of genes of interest using qPCR}

To confirm our RNA-Seq findings using a different assay, we probed 4 genes from the fungus (MAS3, BAS1, aquaporin 9 and BAS3) and 5 genes from the plant (OSFKBP65, LOC_Os06g37224, cytochrome P450, PR1b and $\beta$-1,3-glucanase) using qPCR. Expression levels were highly correlated across all targets, though the magnitude was slightly higher in RNA-seq than qPCR. Results of qPCR analysis are presented in Figures 3.10 and 3.11. Details on the primer probe sets for these genes are provided in (Additional file S9).

\section{Discussion}

Although temperature has long been reported to be an important component of plant disease development, at present knowledge on the mechanisms underlying the effect of elevated temperature on plant pathogen interaction is limited. Therefore, it is important to gain indepth knowledge about the complexity of transcriptional regulation in $M$. oryzae and rice 
during high temperature stress using advanced technologies. We performed RNA-seq analysis to explore the transcriptional variations in $M$. oryzae and rice at $35^{\circ} \mathrm{C}$ and $28^{\circ} \mathrm{C}$ in an attempt to understand fungal pathogenic and plant defense mechanisms modulated during infection at high temperature. Interestingly, in planta fungal biomass was significantly higher in Nip35i than Nip28i, suggesting that by $48 \mathrm{hpi}$, in planta fungal biomass was consistent with the rate of lesion expansion observed in Nip35i. This result suggested that high temperature increased the rate of colonization of the plant tissues by the pathogen. Thus, it was critical to identify genetic factors that facilitate rapid colonization of the host and the corresponding genetic responses exhibited by the plant host against the pathogens at HT. Therefore, an RNA-Seq analysis was carried out to gain a deeper understanding of the genes that are regulated during host pathogen interaction at high temperature. We identified a total of 5377 differentiallyregulated transcripts from $M$. oryzae, 4302 of which were commonly regulated at both temperatures, suggesting that transcriptional signatures in Nip35i and Nip28i were largely similar. However, most up-regulated fungal genes were stronger induced in Nip35i than Nip28i. A stronger transcript accumulation during infection at $35^{\circ} \mathrm{C}$ suggested that there was potentially increased protein synthesis activity to facilitate pathogen adaptation to the host environment, which was probably altered by high temperature treatment.

Given that fungal induction of virulence factors is necessary for successful colonization of the host, we searched for pathogenicity related genes in the PHI database. Many pathogenicity genes previously reported, including MAS3 (MGG_12337), PWL2, PWL1, ribonuclease T2, MoHox7, AVR-Pita and (BAS1-4) (Kawahara et al., 2012) were induced, but with a stronger induction in Nip35i compared to Nip28i. In addition, two genes, MGG_16585 and MGG_15972 with 99\% partial sequence identity to Avrpiz-t and AvrPik/kp/km, respectively, were stronger induced during infection at $35^{\circ} \mathrm{C}$ than at $28^{\circ} \mathrm{C}$. MAS3 is tentatively assigned to GH125 family of $\alpha$-mannosidases in the CAZy database. Fungal cell walls contain high levels of mannoproteins (glycosylphosphatidylinositol anchored) which are synthesized through glycosylation by addition of mannose to their serine or threonine amino acid residues through mannosyltransferase activity, and further processing by mannosidases (Oh et al., 2008). The entire process aids in stabilizing the cell wall either during invasive growth or during appressorium expansion. The high induction of MAS3 at both temperatures might suggest that this gene is crucial to M. oryzae invasive growth. Mannose uptake and utilization is also likely to be controlled by MGG_00994. MGG_00994 is a secreted alpha-1,2-mannosidase capable of hydrolyzing alpha-1,2 linked mannobiose. Although this gene was previously shown not to 
be important for M. oryzae pathogenicity (Zhou et al., 2009), its expression in concert with other secreted proteins suggests that it probably plays a role in the modification of fungal cell walls during invasive growth in rice plants.

The role of pathogenicity effectors: Avr-Pita, Pwl1, Pwl2, Avr-Piz-t and AvrPik/kp/km is well documented (Fernandez and Wilson, 2014a). We found increased transcript levels of genes encoding for these effectors at elevated temperature, which suggests that increased levels of these effectors may be required for pathogenesis at elevated temperatures. Ribonuclease T2 enzymes are reported to be involved in turning over ribosomal RNA to maintain cellular homeostasis, tRNAs cleavage, pathogenicity, phosphate scavenging and nitrogen storage (MacIntosh, 2011). In M. oryzae, tRNAs cleavage was recently proposed to restrict protein synthesis during appressorium formation in order to direct cellular metabolism towards colonization (Nunes et al., 2011). Although the role of ribonuclease T2 in conferring such functions remains to be understood, the increased expression of its encoding gene may imply a potential role in enhanced protein synthesis during colonization.

Several secreted putative effectors, previously reported (Mathioni et al. 2011; Kawahara et al. 2012) were more induced at $35^{\circ} \mathrm{C}$ than $28^{\circ} \mathrm{C}$. Among which, MGG_00230, which shares $98 \%$ partial sequence identity with AVR-Pita2 was strongly induced in both Nip35i and Nip28i. Furthermore, three secreted protein encoding genes with LysM domains, MGG_03468, Slp1 (MGG_10097) and MGG_02405, were induced in both Nip35i and Nip28i. Slp is crucial for suppression of PAMP triggered immunity (Zhang and $\mathrm{Xu}, 2014$ ). Protein sequence alignment indicated more conserved signatures between MGG_03468 and Slp1. However, Slp1 had more deletions than MGG_03468 and MGG_02405, indicating that it may be a novel derivative different from the other two. Nevertheless, the high induction of MGG_03468 and Slp1 suggests synergistic or complementary roles in suppressing PAMP triggered immunity.

Phylogenetic relationship of the top 50 secreted proteins in this study formed three major clusters, indicating a possible similarity between the known and unknown secreted proteins. Several of these proteins could potentially represent new virulence factors active in host plant colonization. For instance, MGG_11009 is a putative cell wall galactomanno (GM) protein, which shares $99 \%$ identity with hydrophobic surface binding protein A (HsbA). Hydrophobins form protective surface coatings for fungi and reduce water surface tension, both of which are needed for growth delimitation of aerial structures such as hyphae and 
conidiospores (Ohtaki et al., 2006). The same authors demonstrated that HsbA recruits CutL1 to the PBSA surface for consequent hydrolysis. Cutinases were previously shown to be necessary for carbon acquisition during subcuticular growth of Venturia inaequalis (Koller et al., 1991). Although the link between HsbA and cutinases is not clear, we speculate that in a stressful environment, there is a possibility of interaction between the two proteins. Moreover, cutinases are probably crutial to $M$. oryzae pathogenicity at high temperature. GMs have also been reported to exist in a glycosylphosphatidylinositol membrane-bound form (Morelle et al., 2005), which might provide a stable association with the lipid bilayer during infection. Another gene, MGG_14055, which shares sequence similarity with genes encoding for $\beta$ Xylosidases was strongly induced at $35^{\circ} \mathrm{C}$. Xylans are the most abundant hemicelluloses in plants. The complete degradation of hemicellulose requires complementary activity of hemicellulolytic enzymes, including endo-1, 4-L-xylanases, for the hydrolysis of xylan backbone, and L-xylosidases for the cleavage of xylooligomers to free xylose (Czjzek et al., 2005). The expression of $\beta$-Xylosidases in Nip35i might suggest a probable form of a backup system for the pathogen to degrade the plant cell wall at high temperature.

To further clarify the differences between $M$. oryzae infection at $35^{\circ} \mathrm{C}$ and $28^{\circ} \mathrm{C}$, we looked for genes encoding for enzymes involved in lipid metabolism. Several genes involved in the initial step of fatty acid $\beta$-oxidation, including MFP1, ICL, PTH2, MgPex6, ECH1 and lipases were more induced in Nip35i than Nip28i. Initial steps of infection may depend on metabolism of stored lipids via the glyoxilate pathway to produce glucose that supports fungal development. The induction of $\beta$-oxidation genes suggests generation and conversion of acetyl CoA to canitine intermediates for energy production during host invasion. Indeed, PTH2 was previously reported to be required for M. oryzae pathogenesis, whereas MgPEX6 was reported to be necessary for lipid body degradation during pathogenesis (Reviewed in Fernandez and Wilson, 2014a). Moreover, ECH1 was reported to be important in neutralizing host defenses at post-penetration stage. Increased expression of genes encoding for these proteins at high temperature suggests that their transcript accumulation is necessary for $M$. oryzae infection at high temperature. Alternatively, the role of $M$. oryzae lipases in pathogenicity is intriguing. Deletion of individual lipase encoding genes has been reported to be less effective in reducing M. oryzae pathogenesis (Wang et al., 2007). However, their induction in this study may be linked to enhanced biomass accumulation during M. oryzae infection at high temperature as previously reported (Maia et al., 1999). We also detected a strong induction of LPP. LPPs have been reported to be involved in dephosphorylation of 
lysophosphatidic acid (LPA), sphingosine 1-phosphate (S1P) and related substrates to control intracellular lipid metabolism and signaling (Sigal et al., 2005). The high induction of this gene is likely crutial to $M$. oryzae lipid biosynthesis during infection.

Several genes involved in carbohydrate metabolism, including GH43, GH7, GH10, PL1, PL4 and CEs were less affected by high temperature, and showed similar induction on Nip35i and Nip28i. Of particular interest was the induction of CEs and PLs. The induction of genes encoding for these enzymes likely contributes to deacetylation of chitin and degradation of pectin, respectively, during $M$. oryzae infection. However, increased expression levels of genes involved in the utilization of non-preferred carbon sources was detected in Nip35i, and may probably suggest disperate measures by the pathogen to ensure rapid colonization of the host. For instance, cellobiose dehydrogenase $(\mathrm{CDH})$ is an extracellular hemoflavoenzyme produced by several cellulose degrading fungi (Baminger et al., 1999), whereas alcohol dehydrogenase I (MGG_03880), aldehyde dehydrogenase (MGG_00719) and a NADPdependent alcohol dehydrogenase 6 (MGG_00246), are involved in ethanol degradation for the production of acetyl-CoA. Due to reduced photosynthetic activity in the plants in response to high temperature, it is possible that a shift in the glycolytic pathway could occur to produce ethanol. This shift likely plays a significant role during infection, and is consistent with the strong induction of ICL and malate synthase, the two enzymes that are necessary for glyoxylate shunt. Increased expression of stress response genes was also detected in this study, including genes involved in alternative pathways for detoxification, and secondary metabolism. Excessive ROS production in the fungal cells has been reported to induce accumulation of aldehydes and alcohols (Asiimwe et al., 2012). The induction of alcohol dehydrogenases and aldehyde dehydrogenases could also suggest a detoxification strategy instead of nutrient aquisition from alcohols and aldehydes.

Increased transcript levels of GUT1, which is a key regulator in the conversion of glycerol to glycerol-3-phosphate (G3P) was detected among in planta expressed genes. Previous reports have shown that fungal glycerol metabolism during host invasion plays an important role in the establishment of infection, including growth and conidiation in planta (Venugopal et al., 2009). Both glycerol and mannose may be a source of carbon in planta, in a likely event of a shift in the glycolytic pathway, and is consistent with a stronger induction of manose utilization enzymes previously discussed. This is also consistent with the lower induction of hexose uptake protein (HUP1) in Nip35i, which could be a strategy for rapid transition to 
necrotrophy during stress. As discussed before, high temperature probably invokes reorganisation of energy supplies in the plant thereby reducing nutrient availability to the pathogen at the biotrophic phase forcing the fungus to rapidly activate necrotrophic phase processes, which dramatically induces necrosis, as observed in Nip35i diseased phenotypes.

Stress related genes, including genes involved in secondary metabolism were also more induced in Nip35i than Nip28i. For instance, averantin oxidoreductase, a key enzyme in the hydroxylation of averantin during aflatoxin biosynthesis; pisatin demethylase, which detoxifies pisatin and functions as virulence factor in Fusarium solani (Khan et al. 2003); and epoxide hydrolases, which have been reported to be enhanced at acidic $\mathrm{pH}$ and glucose (Morisseau and Hammock, 2013) were strongly induced in Nip35i. In addition, metalloproteinases, which deactivate proteinaceous substrates and contribute to cell wall degradation by targeting structural proteins such as hydroxyproline-rich glycoproteins (Dunasevsky et al., 2007) were strongly induced in Nip35i. The induction of these genes at high temperature suggests that a similar strategy could be employed by $M$. oryzae during host colonization. Several other genes in cytochrome P450 gene families, including CYP563A1 and CYP608A1, were stronger induced in Nip35i than Nip28i, suggesting enhanced detoxification activity in $M$. oryzae during host colonization at high temperature.

Interestingly, several genes encoding for enzymes involved in ethylene and gibberellin synthesis were also detected. For instance, ent-kaurene oxidase (MGG_02294), which catalyzes oxidation of the 4-methyl group of ent-kaurene producing kaurenoic acid, a key step in gibberellin (GA) biosynthesis, and 2-oxoglutarate-dependent ethylene/succinate-forming enzyme participates in ethylene biosynthesis were highly induced during $M$. oryzae infection at both temperatures. Induction of these genes might as well suggest a counter defense strategy against plant induced defence pathways associated with these molecules. Similarly, SA degradation enzyme encoding genes were strongly induced, which, as well, suggests a dampening effect on SA signaling.

Among the $\mathrm{ABC}$ transporters, an orthologue of CDR1 was strongly induced at both temperatures. $\mathrm{ABC}$ transporters are known to detoxify antifungal compounds produced by the plant cells, and a strong induction of CDR1during $M$. oryzae infection is consistent with previous reports (Mathioni et al., 2011). Some ABC transporters may facilitate the aquisition of nutrients from the host during in planta growth, and this may be consistent with the 
induction of $\mathrm{ABC}$-type $\mathrm{Fe}^{3+}$ transport system in this study. Alternatively, the higher induction of MFS encoding genes in Nip35i than in Nip28i indicates that these monooxygenases may take part in intracellular pathways required for temperature adaptation or in detoxification of plant derived antifungal molecules. MFS proteins have been implicated in transmembrane transport of a wide range of substrates (Law et al., 2008). Similarly, a strong induction of genes encoding for aquaporin water channels that prevent osmotic damage of cells suggests potential induction of mechanisms leading to dessication tolerance during M. oryzae inplanta growth.

To colonize host plants successfully, pathogens must overcome the ROS toxic environment in the plant. Most antioxidative enzymes were less induced in this study, and is consistent with previous reports (Egan et al., 2007). However, we detected a higher induction of MoCCP2 (MGG_10368), MoVPX1 (MGG_02210) and CATA (MGG_10061) in Nip35i than Nip28i. Alternatively, MoHPX2 was highly induced in Nip28i but was not significantly induced in Nip35i. The differential induction of these peroxidases suggests that antioxidation is regulated by temperature stress during $M$. oryzae infection. Coupled with this, the differential enrichment of casein kinase II site, which flanks di-leucine motifs that are present in transmembrane proteins in humans (Mauxion et al., 1996), in genes specifically induced in Nip35i suggest alternative antioxidative pathways in M. oryzae. Di-leucine-based motifs and tyrosine motifs mediate endocytosis of cell surface proteins. Moreover, tyrosine phosphorylation motifs are present in protein kinase $\mathrm{C}(\mathrm{PKC})$, which is a key mediator of ROS activation of protein kinase D leading to cell survival under oxidative stress (Döppler and Storz, 2007). This signaling pathway could be important for survival under oxidative stress in M. oryzae.

The enrichment of N-glycosylation in Motif 3 was consistent with enrichment of the KEGG pathway, N-Glycan biosynthesis and the higher induction of asparagine synthase in Nip35i than Nip28i. $N$-linked glycans attached to nitrogen of asparagine requires participation of dolichol phosphate for N-lycosylation, which is consistent with the induction of dolichyl lipids in Nip35i. N-glycosylation confers stability or correct folding of secreted glycoproteins, and likely plays a key role during infection at high temperature.

A large number of $\mathrm{TF}$ genes were commonly regulated, suggesting that the same transcription regulators probably control infection, although the level of induction varies with the external 
plant stress environment. Among the transcription factors identified in this study, a homeodomain like (MGG_09628), which was previously reported (Kawahara et al., 2012), showed the strongest induction at both temperatures, whereas a nucleic acid-binding, OB-fold (MGG_04335) was strongly induced in Nip35i. In addition, two Zn2Cys6 genes (MGG_02879 and MGG_00049) and C2H2 zinc finger (MGG_04546) were >10-fold induced in Nip35i, but not in Nip28i, suggesting that these TFs are probably crucial to M. oryzae adaptation to elevated temperature during host colonization.

To explore how rice transcriptome is altered in response to both $M$. oryzae and temperature, we searched for genes involved in various biological pathways in our dataset. The GO analysis exhibited common enrichment in metabolism, response to stress and response to stimuli in both high temperature and M. oryzae stress response genes. A clear difference observed in this study was the rapid symptome development in Nipponbare at high temperature, as opposed to our previous findings with $\mathrm{R}$ genes (Onaga et al., unpublished). We previously reported that high temperature might offer the rice plants an advantage by priming SA conversion to MeSA which prods the JA and ET induction. In this study, most genes involved in hormonal pathways, including OsBISAMT1 were up-regulated. However, we found that genes encoding for jasmonate O-methyltransferase were not significantly expressed in Nipponbare. The same was true for naringenin 7-O-methyltransferase (NOMT; LOC_Os12g13800), a positive regulator of sakuranetin biosynthesis (Shimizu et al. 2012), which is regulated by JA (Rakwal et al., 1999). NOMT was induced in Nip28i but was not detected in Nip35i suggesting that high temperature compromised its expression. Consistent with this, LOC_Os09g17560, previously reported as one of the genes encoding for OsOMT involved in sakuranetin biosynthesis (Park et al., 2013) was also missing. Incidentally, the absence of these genes corresponded with the strong induction of $M$. oryzae $\mathrm{ABC}$ transporter (ABC1; MGG_13624), previously reported to be strongly induced by azole fungicides and the rice phytoalexin sakuranetin (Oh et al., 2008), in Nip35i but not in Nip28i. Given that $A B C 1$, which is responsible for regulating intracellular levels of cytotoxic or xenobiotic compounds, was more induced at elevated temperature, the suppression of OsOMT genes in Nip35i could have probably compromised resistance in Nip35i, The same was found for a multiprotein bridging factor 1c (MBF1c) which was strongly induced in our previous study. Moreover, heat shock transcription factor A2 (HsfA2) which was strongly induced in our previous study, was equally suppressed in Nipponbare, further suggesting compromised stress response. We also studied ABA associated callose deposition genes. Although OsBBD1 
Chapter 3: Transcript profiles of Magnaporthe oryzae in planta expressed genes and rice defence response genes in plants pre-exposed to high temperature

(LOC_Os01g50622), which is involved in abscisic acid-derived callose deposition was significantly induced in this study, we speculate that callose deposition alone may not stop the fungus from colonizing the rice plants. Besides this, two Homeobox genes (LOC_Os09g15480 and Hox24; LOC_Os02g43330), which were highly induced in LT in our previous study were not detected in Nipponbare.

On the other hand, a stronger induction of expansins in this study compared to our previous study likely affected the integrity of the cell wall. In fact expansins were previously reported to increase rice susceptibility to Xanthomonus oryzae (Ding et al., 2008). GH3.8 (LOC_Os07g40290), which was shown to regulate expression of expansins by the same authors, was not detected in Nipponbare, when compared to our previous study. Remarkably, expansin genes were expressed in concert with pectin methylesterase (LOC_Os09g39760; 61fold) and von Willebrand factor type A (LOC_Os11g45990; 36-fold). Pectin methylesterase is induced in Arabidopsis upon infection and is necessary for a successful colonization by necrotrophic pathogens (Raiola et al., 2011). These cell wall modifications at high temperature likely favored host colonization, and could be compounded by pathogen induction of polygalacturonase A (MGG_08938) and pectate lyase B genes during infection. A significant alteration in transcription factor genes was also observed. We previously reported a strong induction of OsWRKY55 and OsWRKY32 in LT and CO, respectively, and several other WRKYs reported to play complementary roles in a defense response (Peng et al., 2010). These genes were not significantly induced in Nip35i, suggesting that several downstream genes modulated by these TFs were either not expressed or less induced. This is consistent with the reduction of the transcriptome in Nipponbare by almost one third when compared to our previous study.

Taken together, our data highlight how elevated temperature shapes plant pathogen interaction. We show that elevated temperature increases the rate of colonization of the plant tissues by the pathogen when high temperature treated plants are inoculated with $M$. oryzae and kept at $28^{\circ} \mathrm{C}$ for $24 \mathrm{hpi}$ prior to re-exposure to $35^{\circ} \mathrm{C}$. This is contrary to our previous findings, and indicates that the putative mechanisms that enhance resistance at elevated temperature are largely complex. It is not unexpected that some of the specific defences might play an important role in offsetting resistance suppression at elevated temperatures even at reduced transcript levels, while the non-specific defence system adapts. Thus, identifying stable $\mathrm{R}$ genes and understanding the mechanism associated with stable resistance at elevated 
temperatures could provide further insight into the mechanism relevant for improving rice in a changing climate. Several $M$. oryzae genes were highly induced at high temperature. Thus, blast epidemics are likely to occur in some rice production areas, especially where conducive temperatures for infection alternate with elevated temperatures.

\section{Materials and Methods}

\section{Plant and fungal material preparation}

A compatible Tanzanian fungal strain (TAN211.16) and Oryza sativa L. ssp. japonica cv. Nipponbare were used in this study. TAN211.16 was collected in 2011 from infected rice fields in Kyela, Tanzania. This strain was selected for transcriptome sequencing because it was aggressive on most of the isogenic lines tested. The strain is currently maintained at the Division of Plant Pathology and Crop Protection, Goettingen University, Germany as APP number 1177. The genome of the rice cultivar Nipponbare is sequenced and available as a reference for genomic studies. The plants were grown and maintained in the greenhouse at a temperature of $27 \pm 3^{\circ} \mathrm{C}$ with a photoperiod of 14 hours for 18 days. Prior to inoculation, seedlings were transferred from the greenhouse to the growth chambers maintained at $28^{\circ} \mathrm{C}$ and $35^{\circ} \mathrm{C}$ for additional 7 days. Inoculated plants were kept at $28^{\circ} \mathrm{C}$ in the dark for 24 hours to allow for fungal infection. Thereafter, plants were returned to respective temperature chambers $\left(28^{\circ} \mathrm{C}\right.$ and $\left.35^{\circ} \mathrm{C}\right)$ for additional 24 hours prior to sample collection. We obtaine fungal mycilia by growing the fungus in $250 \mathrm{ml}$ of a medium containing $3 \mathrm{gL}^{-1}$ of glucose, $3 \mathrm{gL}^{-1}$ of casamino acids and $3 \mathrm{gL}^{-1}$ of yeast extract. The culture was incubated at $25^{\circ} \mathrm{C}$ under continuous rotation at $100 \mathrm{rpm}$ for $24 \mathrm{~h}$. The procedure was repeated for additional $24 \mathrm{~h}$ by blending the first culture with a fresh medium. Mycelium was collected by filtration and stored at $-80^{\circ} \mathrm{C}$ for RNA extraction. Inoculation and symptom evaluation was carried out as previously described (Onaga et al., 2015).

\section{Sample preparation mRNA extraction and sequencing}

Infected leaves from each treatment were sampled from four plants in two replicates and immediately frozen in liquid nitrogen. Leaf samples infected with $M$. oryzae at $28^{\circ} \mathrm{C}$ and $35^{\circ} \mathrm{C}$ are designated as Nip28i and Nip35i, respectively. Samples collected before inoculation are designated as Nip28c, Nip35c and Fungus. Infected leaf samples were collected at 48 hours post inoculation (hpi). We chose to examine $48 \mathrm{hpi}$ because this is the most likely stage at which several fungal effectors, including those that culminate into effector triggered 
immunity (ETI) are abundantly secreted. Total RNA was extracted with Trizol reagent (Invitrogen, Carlsbad, CA) according to the manufacturer's instructions. Four micrograms of total RNA were subjected to library preparation using the "TruSeq RNA Sample preparation v2 Guide" following the manufacturer's instructions. Libraries were amplified by 15 cycles of PCR, and concentration and size were assayed on a 2100 Bioanalyzer (Agilent). Libraries were clustered for sequencing using Illumina cBOT Cluster generation system and TrusSeq v3 chemistry. Fifty bp single-end sequencing was performed on the HiSeq2000. Sequence images were transformed with Illumina software BaseCaller to bcl files, which were demultiplexed to fastq files with CASAVA v1.8.2 allowing for one mismatch in the indices. The mixed transcriptome was alligned to the respective genomes of both organisms using Bowtie2 v2.0.2, first to the rice genome followed by $M$. oryzae genome. The reads that did not map on to the rice genome were considered to have come from $M$. oryzae, and were mapped onto the reference genome of isolate 70-15. Unmapped $M$. oryzae reads were recombined with the mapped read SAM files to guide assembly, and later processed with the de-novo assembler using CLC Genomics Workbench. The resulting sam files from the respective genome mappings were converted to sorted bam files using samtools 0.1.18. Further processing was conducted as previously described (Onaga et al. unpublished). GO analysis of fungal DEGs was conducted using the DAVID functional annotation tool (Dennis et al., 2003), while plant genes were analyzed using the hypergeometric statistical method with Hochberg FDR adjustment in the AgriCO website (http://bioinfo.cau.edu.cn/agriGO/analysis.php webcite) as described elsewhere (Du et al., 2010). Upstream protein sequences were analyzed for enriched motifs using the MEME algorithm. Motifs were visualized with Weblogo (Crooks et al., 2004). Maximum Likelihood based phylogenetic relationship between protein sequences of genes encoding putative secreted proteins were analysed on the Phylogeny.fr platform (Dereeper et al., 2008).

\section{Relative real-time qRT-PCR}

To confirm the results of RNA-seq analysis, we determined the expression levels of 10 DEGs by qRT-PCR. Total RNA $(1.0 \mu \mathrm{g})$ was used to synthesize cDNA using the iScript ${ }^{\mathrm{TM}}$ Reverse Transcription Supermix for RT-qPCR (BioRad). Following the instruction manual, the reverse transcription conditions were: $5 \mathrm{~min}$ at $25^{\circ} \mathrm{C}, 30 \mathrm{~min}$ at $42{ }^{\circ} \mathrm{C}$ and finally $5 \mathrm{~min}$ at 85 ${ }^{\circ} \mathrm{C}$. Primers were designed using the software Primer3Plus (http://www.bioinformatics.nl/cgibin/primer3plus/primer3plus.cgi/). Candidate reference genes [(actin, beta tubulin, elongation 
factor and expressed protein (LOC_Os07g02340)] were selected from previous reports (Narsai et al., 2010), and tested for stability. The reference genes, LOC_Os07g02340 (for rice) and Actin (for the fungal genes), were used in subsequent RT-qPCR reactions based on their stable expression in all treatments. Gene-specific primers were used for amplification of the target genes using iQ SYBR Green supermix on the thermal cycler CFX384 (BioRad) under the following conditions: $3 \mathrm{~min}$ at $95{ }^{\circ} \mathrm{C}(1 \mathrm{cycle}), 15 \mathrm{~s}$ at $95{ }^{\circ} \mathrm{C}$ and $5 \mathrm{~s}$ at $62{ }^{\circ} \mathrm{C}(50$ cycles) and a melting curve from 60 to $95^{\circ} \mathrm{C}$ in triplicates (all from Bio-Rad). The relative expression was computed as previously described (Pfaffl, 2001). Fungal quantification was performed using primers reported in Harmon et al. (2003), which amplify a 687bp region of repetitive DNA fragment Pot2. About 100 copies of Pot2 are reported to be present per genome of M. oryzae (Kachroo et al., 1994).

\section{Acknowledgement}

This work was supported by BMZ funded project "Mitigating the impact of climate change on rice diseases in East Africa". We thank the Microarray and Deep-Sequencing Core Facility of the Developmental Biochemistry University Medical Center Göttingen (UMG) for performing the RNA-seq analysis. In particular, we would like to thank Fabian Ludewig and Dr. Gabriela Salinas-Riester for successfully running illumina sequencing, and Dr. Claudia Pommerenke for bioinformatic analysis. Our sincere thanks are expressed to Dagmar Tacke, Evelyn Vorbeck, Marian Süß and Daniel Kretzschmar for their technical support.

\section{References}

Asiimwe, T., Krause, K., Schlunk, I. and Kothe, E. (2012) Modulation of ethanol stress tolerance by aldehyde dehydrogenase in the mycorrhizal fungus Tricholoma vaccinum. Mycorrhiza 22, 471-484.

Baminger, U., Nidetzky, B., Kulbe, K.D. and Haltrich, D. (1999) A simple assay for measuring cellobiose dehydrogenase activity in the presence of laccase. J. Microbiol. Methods, 35, 253-259.

Bendtsen, J.D., Nielsen, H., von Heijne, G. and Brunak, S. (2004) Improved prediction of signal peptides: SignalP 3.0. J. Mol. Biol. 340, 783-795

Bita, C.E. and Gerats, T. (2013) Plant tolerance to high temperature in a changing environment: scientific fundamentals and production of heat stress-tolerant crops. Front.

Plant Sci. 4, 273. 
Budde, I.P. and Ullrich, M.S. (2000) Interactions of Pseudomonas syringae pv. glycinea with host and non-host plants in relation to temperature and phytotoxin synthesis. Mol. Plant-Microbe Interact. 13, 951-961.

Ceccardi, T.L., Barthe, G.A. and Derrick, K.S. (1998) A novel protein associated with citrus blight has sequence similarities to expansin. Plant Mol. Biol. 38, 775-783.

Crooks, G.E., Hon, G., Chandonia, J.M. and Brenner, S.E. (2004) WebLogo: a sequence logo generator. Genome Res. 14, 1188-1190.

Czjzek, M., David, A.B., Bravman, T., Shoham, G., Henrissat, B. and Shoham, Y. (2005) Enzyme-substrate complex structures of a GH39 b-xylosidase from Geobacillus stearothermophilus. J. Mol. Biol. 353, 838-846.

Dean, R., van Kan, J.A.L., Pretorius, Z.A., Hammond-Kosack, K.E., Di Pietro, A., Spanu, P.D., Rudd, J.J., Dickman, M., Kahmann, R., Ellis, J. and Foster, G.D. (2012) The top 10 fungal pathogens in molecular plant pathology. Mol. Plant Pathol. 13, 414-430.

Denancé, N., Sánchez-Vallet, A., Goffner, D. and Molina, A. (2013) Disease resistance or growth: the role of plant hormones in balancing immune responses and fitness costs. Front Plant Sci. 24, 155.

Dereeper, A., Guignon, V., Blanc, G., Audic, S., Buffet, S., Chevenet, F., Dufayard, J.-F., Guindon, S., Lefort, V., Lescot, M., Claverie, J.-M. and Gascuel, O. (2008) Phylogeny.fr: robust phylogenetic analysis for the non-specialist. Nucleic Acids Res. 36, W465-9.

Ding, X., Cao, Y., Huang, L., Zhao, J., Xu, C., Li, X. and Wang, S. (2008) Activation of the indole-3-acetic acid-amidosynthetase GH3-8 suppresses expansin expression and promotes salicylate- and jasmonate-independent basal immunity in rice. Plant Cell 14, $228-240$.

Döppler, H. and Storz, P. (2007) A novel tyrosine phosphorylation site in protein kinase D contributes to oxidative stress-mediated activation. J Biol Chem. 282, 31873-31881.

Doyle, E.L. (2006) Computational and experimental analysis of TAL effector-DNA binding. PhD thesis. Iowa State University, Ames, Iowa.

Du, Z., Zhou, X., Ling ,Y., Zhang, Z. and Su, Z. (2010) agriGO: a GO analysis toolkit for the agricultural community. Nucleic Acids Res. 38, W64-W70.

Dunasevsky, Y.E., Matveeva, A.R., Beliakova, G.A., Domash, V.I. and Belozersky, M.A. (2007) Extracellular alkaline proteinase of Colletotrichum gloeosporioides. Biochemistry, 72, 345-350. 
Egan, M.J., Wang, Z.Y., Jones, M.A., Smirnoff, N. and Talbot, N. J. (2007) Generation of reactive oxygen species by fungal NADPH oxidases is required for rice blast disease. Proc. Natl. Acad. Sci. U S A. 104, 11772-11777.

Fernandez, J. and Wilson, R.A. (2014b) Characterizing roles for the glutathione reductase, thioredoxin reductase and thioredoxin peroxidase-encoding genes of Magnaporthe oryzae during rice blast disease. PLoS ONE 9, e87300.

Fernandez, J. and Wilson, R.A. (2014a) Cells in cells: morphogenetic and metabolic strategies conditioning rice infection by the blast fungus Magnaporthe oryzae. Protoplasma 251:37-47.

Finn, R.D., Mistry, J., Tate, J., Coggill, P., Heger, A., Pollington, J.E., Gavin, O.L., Gunasekaran, P., Ceric, G., Forslund, K., Holm, L., Sonnhammer, E.L.L., Eddy, S.R. and Bateman, A. (2010) The Pfam protein families database. Nucleic Acids Res. 38, D211-D222.

Garrett, K.A., Dendy, S.P., Frank, E.E., Rouse, M.N. and Travers, S.E. (2006) Climate change effects on plant disease: Genomes to ecosystems. Ann. Rev. Phytopathol. 44, 489509.

Giraldo, M.C., Dagdas, Y.F., Gupta, Y.K., Mentlak, T.A., Yi, M., Martinez-Rocha, A.L., Saitoh, H., Terauchi, R., Talbot, N.J. and Valent, B. (2013) Two distinct secretion systems facilitate tissue invasion by the rice blast fungus Magnaporthe oryzae. Nat. Commun. 4, 1996.

Gregory, P.J., Johnson, S.N., Newton, A.C., Ingram, J.S.I. (2009) Integrating pests and pathogens into the climate change/food security debate. J. Exp. Bot. 60, 2827-2838.

Hashioka, Y. (1950) Studies on the mechanism of prevalence of the rice blast disease in the tropics.Technical Bulletin No. 8. Taiwan Agricultural Research Institute.

Huang, D.W., Sherman, B.T. and Lempicki, R.A. (2009b) Systematic and integrative analysis of large gene lists using DAVID bioinformatics resources. Nature Protocols 4, 44-57.

Kall, L., Krogh, A. and Sonnhammer, E.L. (2007) Advantages of combined transmembrane topology and signal peptide prediction - the Phobius web server. Nucleic Acids Res, 35, W429-432.

Kamolsukyunyong, W., Sukhaket, W., Ruanjaichon, V., Toojinda, T. and Vanavichit, A. (2013) Single-feature polymorphism mapping of isogenic rice lines identifies the influence of terpene synthase on brown planthopper feeding preferences. Rice, $\mathbf{6}, 18$.

Kato, H. and Kozaka, T. (1974) Effects of temperature on lesion enlargement and sporulation of Pyricularia oryzae in rice leaves. Phytopathol. 64, 828-830. 
Kawahara, Y., Oono, Y., Kanamori, H., Matsumoto, T., Itoh, T., Minami, E. (2012) Simultaneous RNA-Seq analysis of a mixed transcriptome of rice and blast fungus interaction. PloS One, 7, e49423.

Khan, R., Tan, R., Mariscal, A.G. and Straney, D. (2003) A binuclear zinc transcription factor binds the host isoflavonoid-responsive element in a fungal cytochrome p450 gene responsible for detoxification. Mol. Microbiol. 49, 117-130.

Koller, W., Parker, D.M. and Becker, C.M. (1991) Role of cutinase in the penetration of apple leaves by Venturia inaequalis. Phytopathology, 81, 1375-1379.

Krogh, A., Larsson, B., von Heijne, G. and Sonnhammer, E.L. (2001) Predicting transmembrane protein topology with a hidden Markov model: Application to complete genomes. J. Mol. Biol. 305, 567-580.

Li, P., Bai, B., Zhang, H-Y., Zhou, H. and Zhou, B. (2012) Genomic organization and sequence dynamics of the AvrPiz-t locus in Magnaporthe oryzae. J. Zhejiang Univ Sci B. 13, $452-464$.

Luo, Y., Teng, P.S., Fabellar, N.G. and TeBeest, D.O. (1998) The effects of global temperature change on rice leaf blast epidemics: a simulation study in three agroecological zones. Agri., Ecosys. and Environ. 68, 187-196

MacIntosh, G.C. (2011) RNase T2 Family: Enzymatic properties, functional diversity, and evolution of ancient ribonucleases In: Nicholson, A.W. (ed.), Ribonucleases, Nuc. Acids and Mol. Biol. 26.

Maia, M. de M.D, Morais, M.M.C. de, Morais, Jr. M.A. de, Melo, E.H.M. and de Lima Filho, J.L. (1999) Production of extracellular lipase by the phytopathogenic fungus Fusarium solani FS1. Revista de Microbiologia. 30, 304-309.

Mosquera, G., Giraldo, M.C., Khang, C.H., Coughlan, S. and Valent, B. (2009) Interaction transcriptome analysis identifies Magnaporthe oryzae BAS1-4 as biotrophyassociated secreted proteins in rice blast disease. Plant Cell, 21, 1273-1290

Mathioni, S.M., Belo, A., Rizzo, C.J., Dean, R.A. and Donofrio, N.M. (2011) Transcriptome profiling of the rice blast fungus during invasive plant infection and in vitro stresses. BMC Genomics, 14, 49.

Morisseau, C. and Hammock, B.D. (2013) Impact of soluble epoxide hydrolase and epoxyeicosanoids on human health. Annu. Rev. Pharmacol. Toxicol. 53, 1-3.22

Mauxion, F., Le Borgne, R., Munier-Lehmann, H. and Hoflack, B. (1996) A casein kinase II phosphorylation site in the cytoplasmic domain of the cation-dependent mannose 6phosphate receptor determines the high affinity interaction of the AP-1 Golgi assembly proteins with membranes. J. Biol Chem, 271, 2171-2178. 
Morelle, W., Bernard, M., Debeaupuis, J.P., Buitrago, M., Tabouret, M., and Latgé, J. (2005) Galactomannoproteins of Aspergillus fumigatus. Eukaryot Cell, 4, 1308-1316.

Nakashita, H., Yasuda, M., Nitta, T., Asami, T., Fujioka, S., Arai, Y., Sekimata, K., Takatsuto, S., Yamaguchi, I., Yoshida, S. (2003) Brassinosteroid functions in a broad range of disease resistance in tobacco and rice. Plant J. 33, 887-898.

Nguyen, Q.B., Itoh, K., Vu, B.V., Tosa, Y. and Nakayashiki, H. (2011) Simultaneous silencing of endo-b-1,4 xylanase genes reveals their roles in the virulence of Magnaporthe oryzae. Mol. Microbiol. 81, 1008-1019.

Nunes, C.C., Gowda, M., Sailsbery, J., Xue, M., Chen, F., Brown, D.E., Oh, Y., Mitchell, T.K., Dean, R. A. (2011) Diverse and tissue-enriched small RNAs in the plant pathogenic fungus, Magnaporthe oryzae. BMC Genomics 12, 288.

Oh, Y., Donofrio, N., Pan, H., Coughlan, S., Brown, D., Meng, S., Mitchell, T., and Dean, R. (2008) Transcriptome analysis reveals new insight into appressorium formation and function in the rice blast fungus Magnaporthe oryzae. Genome Biol. 9, R85.

Ohtaki, S., Maeda, H., Takahashi, T., Yamagata, Y., Hasegawa, F., Gomi, K., Nakajima, T. and Abe, K. (2006) Novel hydrophobic surface binding protein, HsbA, produced by Aspergillus oryzae. Appl. and Envi. Microbiol. 72, 2407-2413.

Ou, S.H. (1985) Rice Diseases, 2nd ed. Kew, Surrey, U.K.: Commonwealth Mycological Institute.

Park, S-Y., Choi, J., Lim, S-E., Lee, G-W., Park, J., et al. (2013) Global Expression Profiling of Transcription Factor Genes Provides New Insights into Pathogenicity and Stress Responses in the Rice Blast Fungus. PLoS Pathog. 9, e1003350.

Peng, Y., Bartley, L.E., Canlas, P. and Ronald, P.C. (2010) OsWRKY IIa transcription factors modulate rice innate immunity. Rice, 3: 36-42.

Raiola, A., Lionetti, V., Elmaghraby, I., Immerzeel, P., Mellerowicz, E.J., Salvi, G., Cervone, F., Bellincampi, D. (2011) Pectin methylesterase is induced in Arabidopsis upon infection and is necessary for a successful colonization by necrotrophic pathogens. Mol. Plant-Microbe Interact. 24, 432-440.

Rauyaree, P., Choi, W., Fang, E., Blackmon, B. and Dean, R.A. (2004) Genes expressed during early stages of rice infection with the rice blast fungus Magnaporthe grisea. Mol Plant Pathol. 2, 347-354.

Reyna, N.S. and Yang, Y. (2006) Molecular analysis of the rice MAP kinase gene family in relation to Magnaporthe grisea infection. Mol. Plant-Microbe Interact. 19, 530-540.

Rice, P., Longden, I. and Bleasby, A. (2000) EMBOSS: the European Molecular Biology Open Software Suite. Trends Genet. 16, 276-277. 
Rawlings, N.D., Barrett, A.J. and Bateman, A. (2010) MEROPS: the peptidase database. Nuc. Acids Res. 38, D227-D233.

Sigal, Y.J., McDermott, M.I. and Morris, A.J. (2005) Integral membrane lipid phosphatases/phosphotransferases: common structure and diverse functions. Biochem. $J$. 387, 281-293.

Sweigard, J.A., Carroll, A.M., Kang, S., Farrall, L., Chumley, F.G. and Valent, B. (1995) Identification, cloning, and characterization of PWL2, a gene for host species specificity in the rice blast fungus. Plant Cell, 7, 1221-1233.

Tang, W., Deng, Z. and Wang, Z.Y. (2010) Proteomics shed light on the brassinosteroid signalling mechanisms. Curr. Opinion in Plant Biol. 13, 27-33.

Teng, P.S. (1994) The epidemiological basis for blast management. In: Zeigler RS, Leong SA, Teng PS. (Eds.), Rice Blast Disease. IRRI, CAB Int. 409-433

Ullrich, M., Peñaloza-Vázquez, A., Bailey, A.M. and Bender, C.L. (1995) A modified twocomponent regulatory system is involved in temperature-dependent biosynthesis of the Pseudomonas syringae phytotoxin coronatine. J Bacteriol. 157, 6160-6169.

Valdivia, E.R., Sampedro, J., Lamb, J.C., Chopra, S. and Cosgrove, D.J. (2007) Recent proliferation and translocation of pollen group 1 allergen genes in the maize genome. Plant Physiol. 143, 1269-81.

Venugopal, S.C., Chanda, B., Vaillancourt, L., Kachroo, A. and Kachroo, P. (2009) The common metabolite glycerol-3-phosphate is a novel regulator of plant defense signaling. Plant Signaling \& Behavior, 4, 746-749.

Wang, Z.Y., Soanes, D.M., Kershaw, M.J. and Talbot, N.J. (2007) Functional analysis of lipid metabolism in Magnaporthe grisea reveals a requirement for peroxisomal fatty acid beta-oxidation during appressorium mediated plant infection. Mol. Plant Microbe Interact. 20, 475-491.

Wilson, R.A. and Talbot, N.J. (2009) Under pressure: investigating the biology of plant infection by Magnaporthe oryzae. Nat. Rev. Microbiol. 7, 185-195.

Yi, M., Chi, M.H., Khang, C.H., Park, S.Y., Kang, S., Valent B. and Lee., Y-H. (2009) The ER chaperone LHS1 is involved in asexual development and rice infection by the blast fungus Magnaporthe oryzae. Plant Cell, 21, 681-695.

Zeigler, R.S., Leong, S.A. and Teng P.S. eds (1994) Rice Blast Disease. Wallingford, UK: Commonwealth Agricultural Bureau International.

Zhang, S. and Xu, J-R. (2014) Effectors and effector delivery in Magnaporthe oryzae. PLoS Pathog. 10, e1003826. 
Chapter 3: Transcript profiles of Magnaporthe oryzae in planta expressed genes and rice defence response genes in plants pre-exposed to high temperature

\section{Figures}

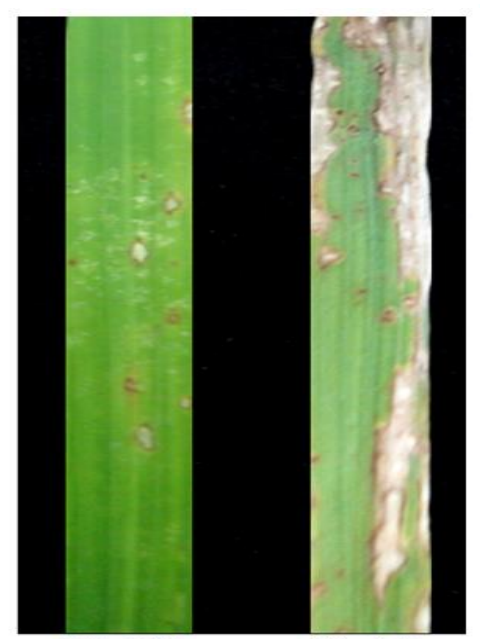

Nip28i

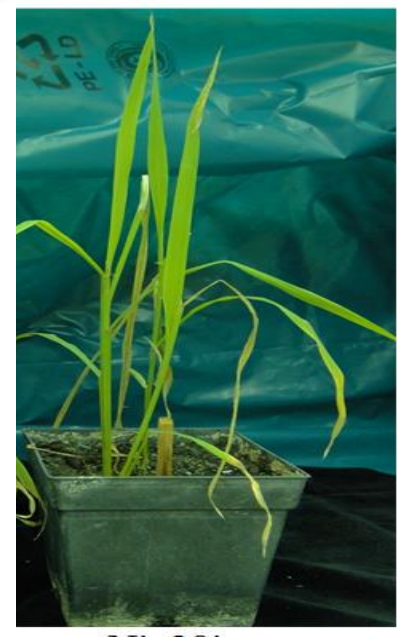

Nip28i

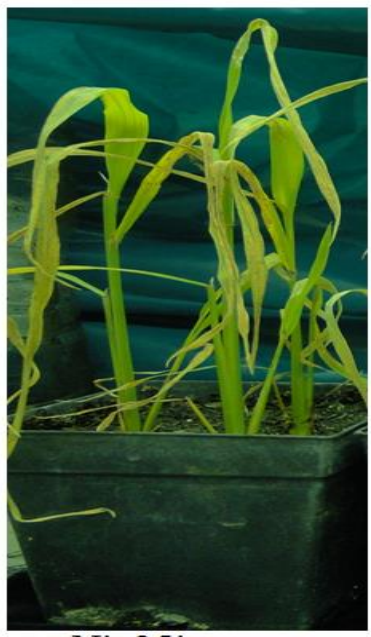

Nip35i

Figure 3.1a: Disease phenotypes of $M$. oryzae on Nipponbare at $28^{\circ} \mathrm{C}(\mathrm{Nip} 28 \mathrm{i})$ and $35^{\circ} \mathrm{C}(\mathrm{Nip} 35 \mathrm{i})$ at 5 dpi. Visible symptomes were observed on Nip35i 3dpi which rapidly enlarged, when compared to Nip28i. Nip35i exhibited rapid collapse of the leaf tissue and enlarged grey brown foliar lesions at 5 dpi. Spores from TAN211.16 were spray-inoculated on Nipponbare (five-leaf stage).

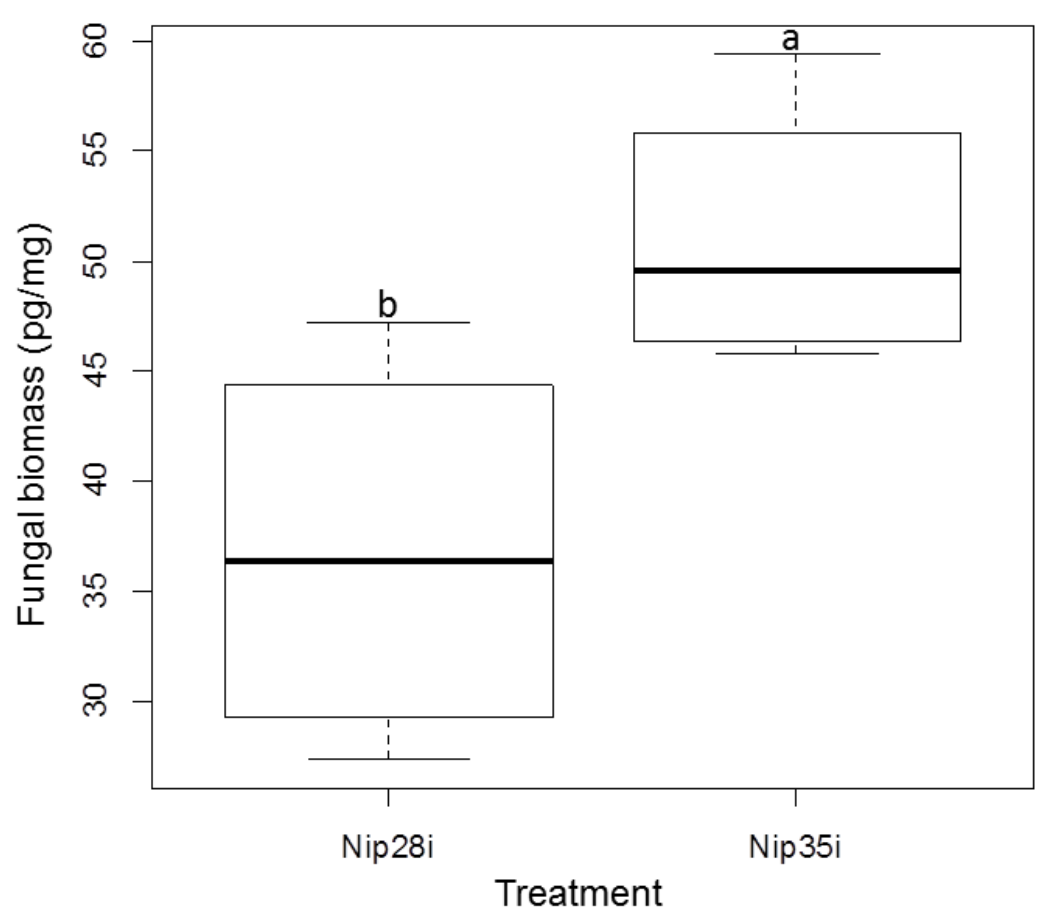

Fig. 3.1b. Quantification of fungal biomass detected by quantitative real-time PCR with primer pair of pfh $2 \mathrm{a}$ and $\mathrm{pfh} 2 \mathrm{~b}$ on rice leaves inoculated with $M$. Oryzae at 48 hpi. Data are presented as mean picograms per miligram (pg/mg) fungal DNA \pm standard deviation (SD) in 2 replicates. Letters indicate significant differences at $\mathrm{p} \leq 0.05$, calculate by Tukey HSD test. 
Chapter 3: Transcript profiles of Magnaporthe oryzae in planta expressed genes and rice defence response genes in plants pre-exposed to high temperature

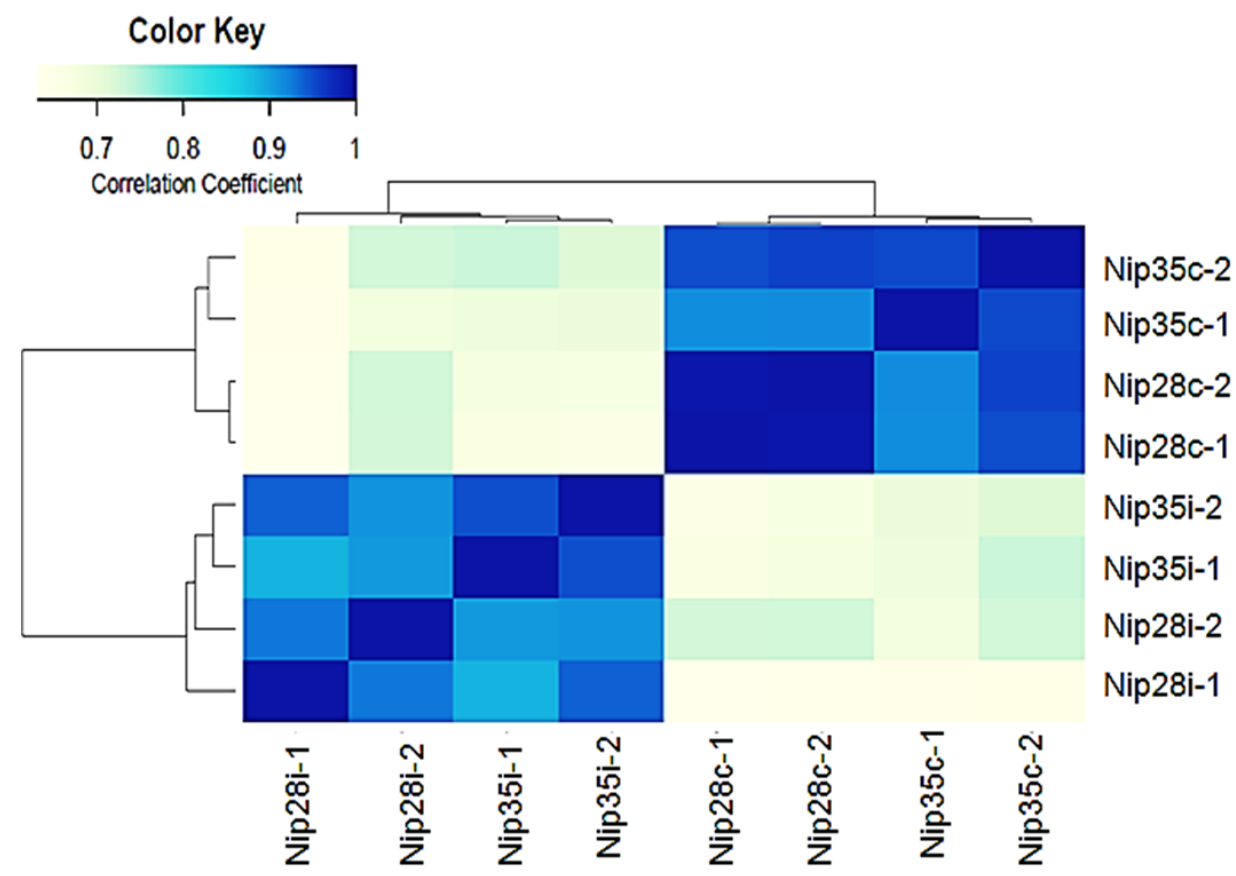

Figure 3.2: Heatmap showing clustering by Euclidean distances between sequenced sample libraries. The darker the color, the closer the two datasets are together. Nip28i and Nip35i represent infected samples at $28^{\circ} \mathrm{C}$ and $35^{\circ} \mathrm{C}$ respectively. Nip28c and Nip $35 \mathrm{c}$ represent uninfected samples at $28^{\circ} \mathrm{C}$ and $35^{\circ} \mathrm{C}$.

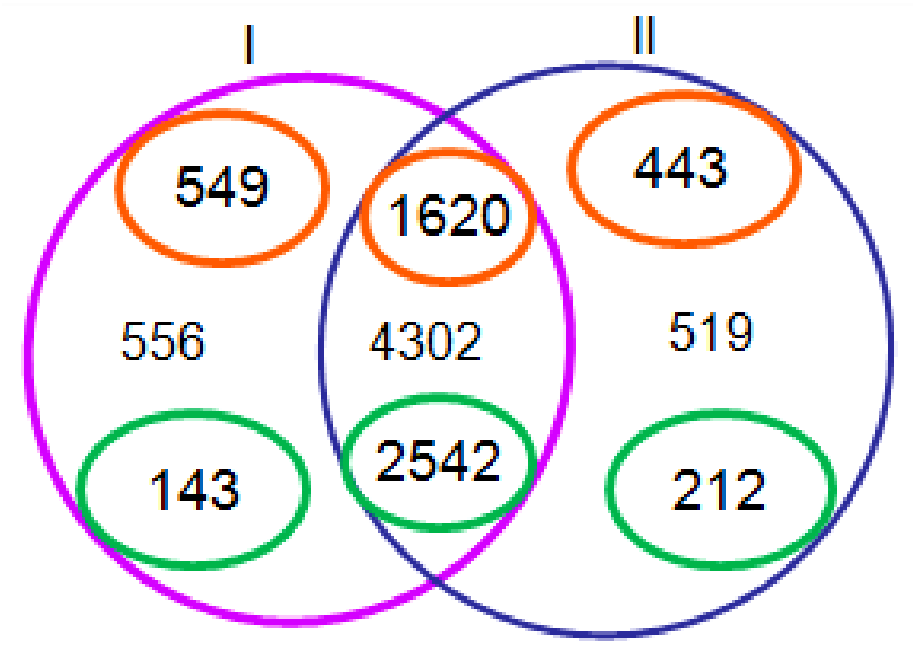

Figure 3.3a: Venn diagram illustrating overlaps between numbers of significant differentially expressed fungal genes (fold change 4; FDR, 0.05) in (I) Nip35i (II) Nip28i. The numbers in the orange circles represent the specific and shared up regulated genes in the respective treatments while those in the green circles represent specific and shared down regulated genes. 
Chapter 3: Transcript profiles of Magnaporthe oryzae in planta expressed genes and rice defence response genes in plants pre-exposed to high temperature

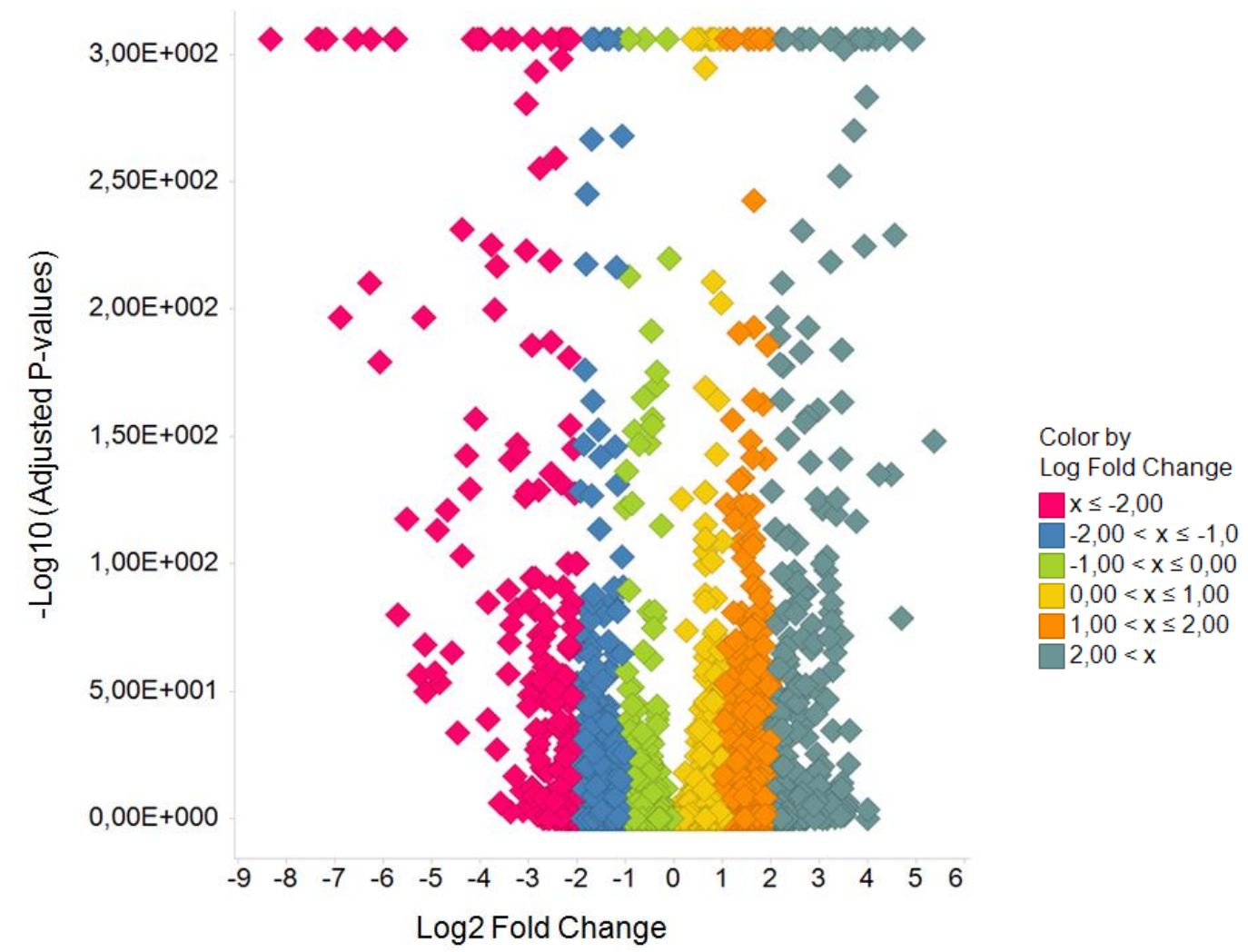

Figure 3.3b: Volcano plot of significance against fold change in fungal in planta gene expression, in Nip35i versus Nip28i. The negative $\log _{10}$ of the adjusted $P$ values is plotted against the fold change difference of $\log _{2}$-normalized expression values. Small expression differences are located at the centre of the plot and log fold changes $\geq 2$ are located outer part of the plot (see legend). Test-wise threshold of $P=0 \cdot 05$ was used (corresponding to Fisher's exact test).

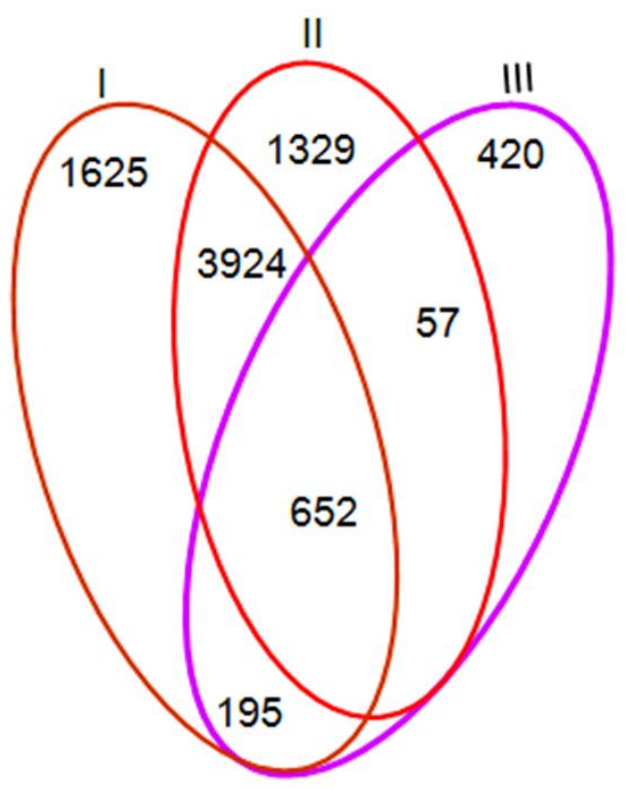

Figure 3.3c: Venn diagram illustrating overlaps between number of significant differentially expressed plant genes (fold change 4; FDR, 0.05) in (I) Nip35i, (II) Nip28i, (III) Nip35c. 
Chapter 3: Transcript profiles of Magnaporthe oryzae in planta expressed genes and rice defence response genes in plants pre-exposed to high temperature

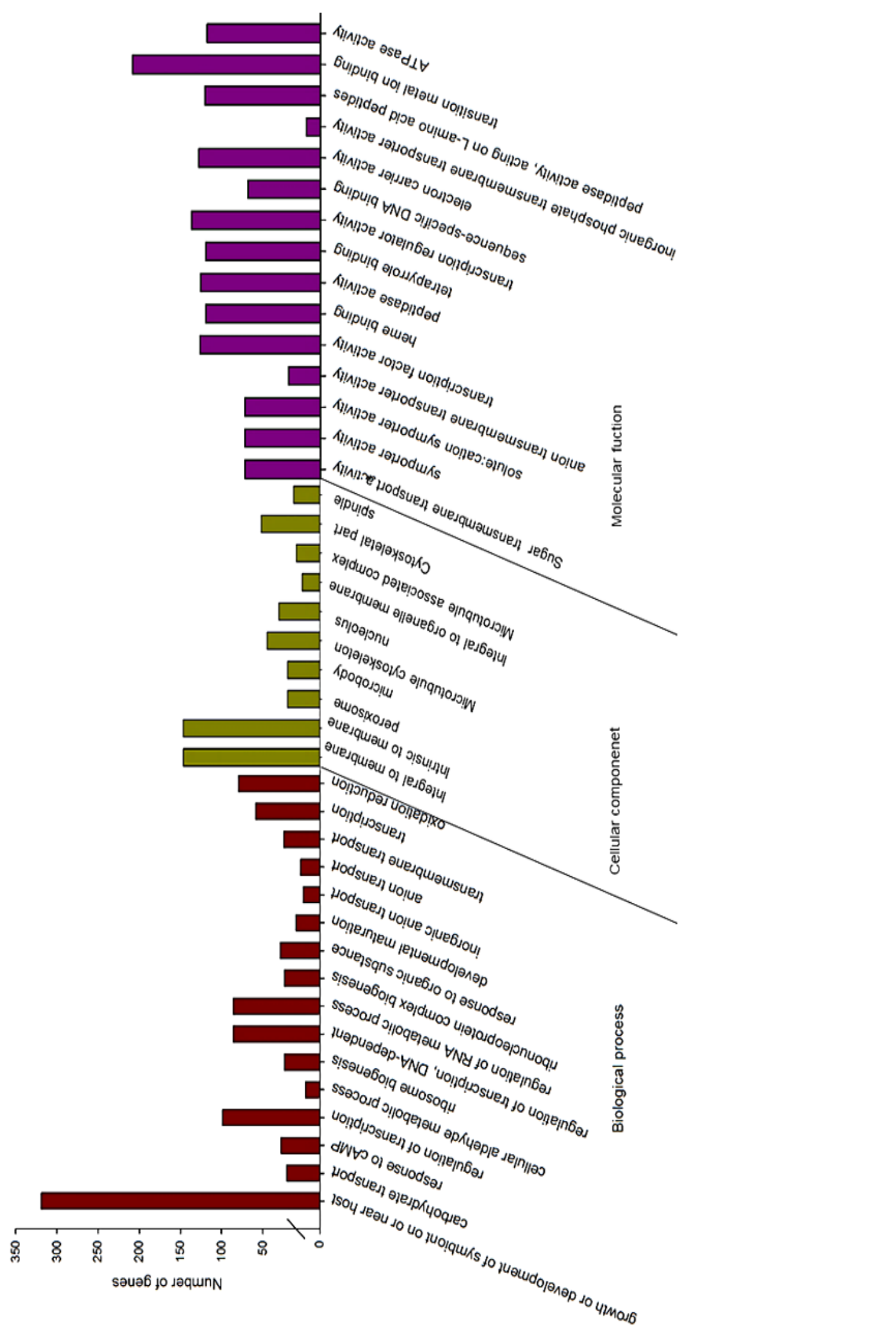

Figure 3.4: Gene Ontology classification of up regulated fungal DEGs in Nip35i (A), categorized into 41 and 39 functional groups of three main categories, respectively: biological process, cellular component and molecular function. 
Chapter 3: Transcript profiles of Magnaporthe oryzae in planta expressed genes and rice defence response genes in plants pre-exposed to high temperature

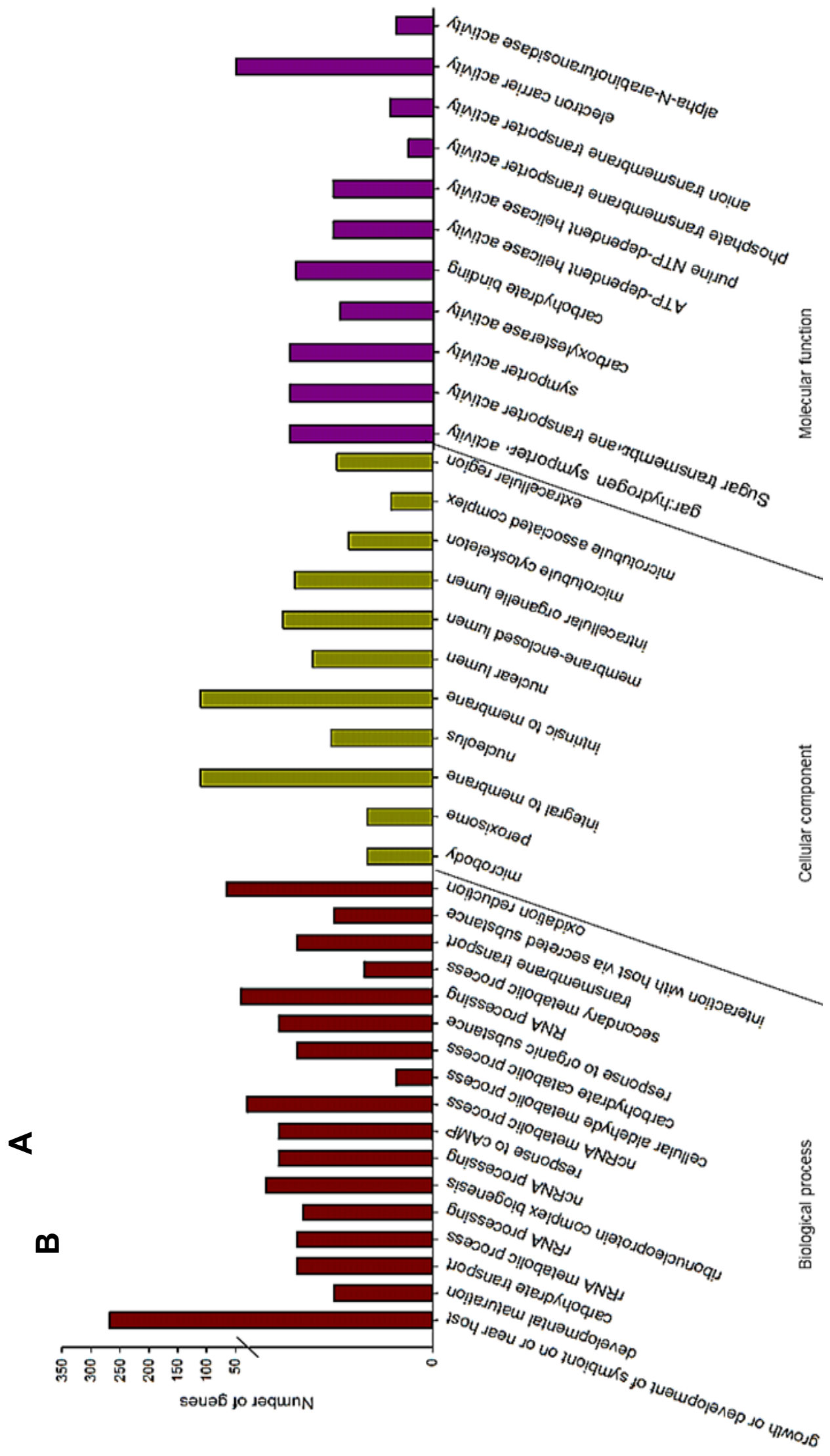

Figure 3.4: Gene Ontology classification of up regulated fungal DEGs in Nip28i (B), categorized into 41 and 39 functional groups of three main categories, respectively: biological process, cellular component and molecular function. 
Chapter 3: Transcript profiles of Magnaporthe oryzae in planta expressed genes and rice defence response genes in plants pre-exposed to high temperature

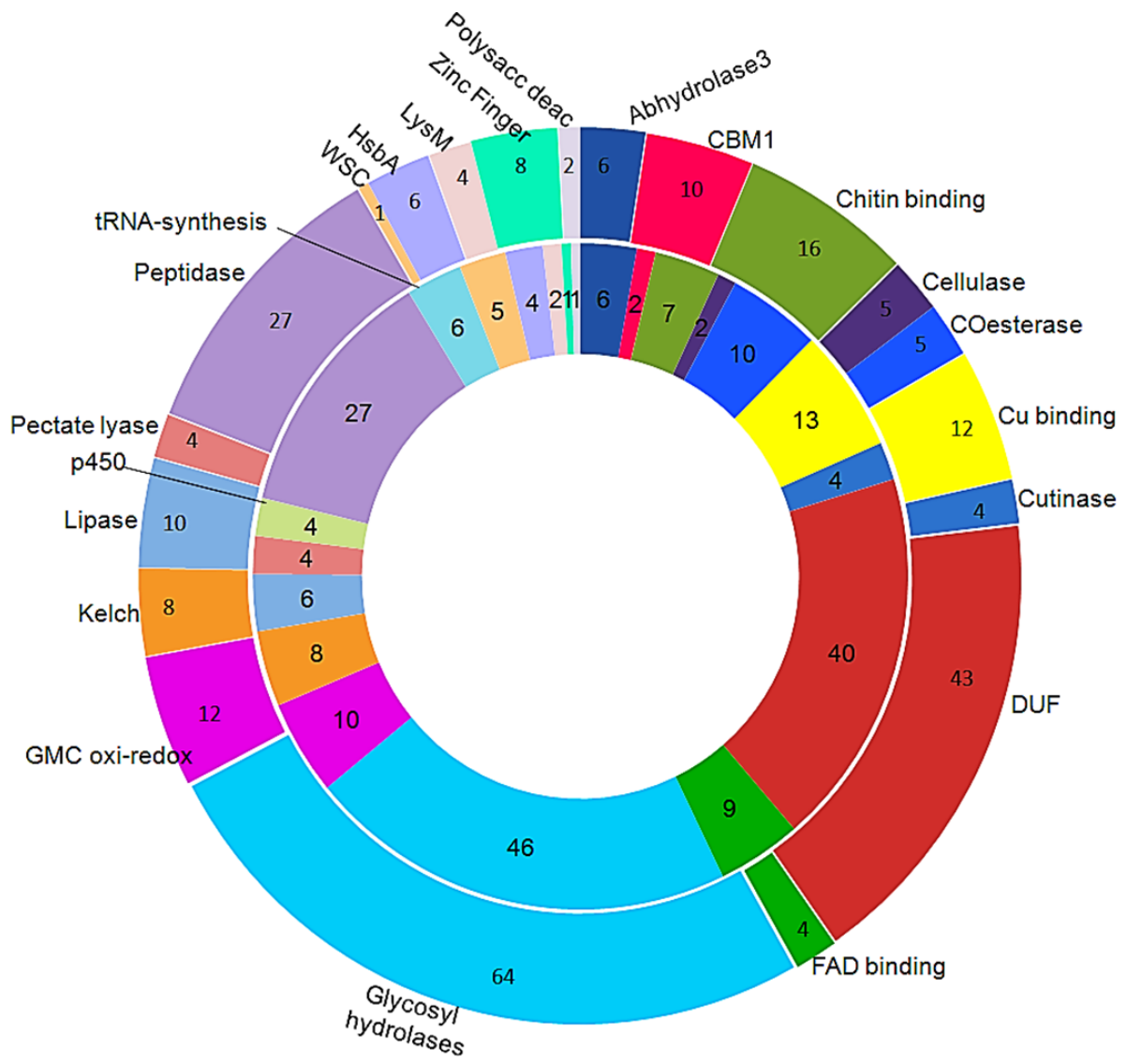

Figure 3.5: Domain classification and comparison of Nip35i (inner circle) and Nip28i (outer circle) putative secreted proteins. In each circle the relative fraction of protein domains (numbers) present in the predicted secreted proteins is presented. 
Chapter 3: Transcript profiles of Magnaporthe oryzae in planta expressed genes and rice defence response genes in plants pre-exposed to high temperature

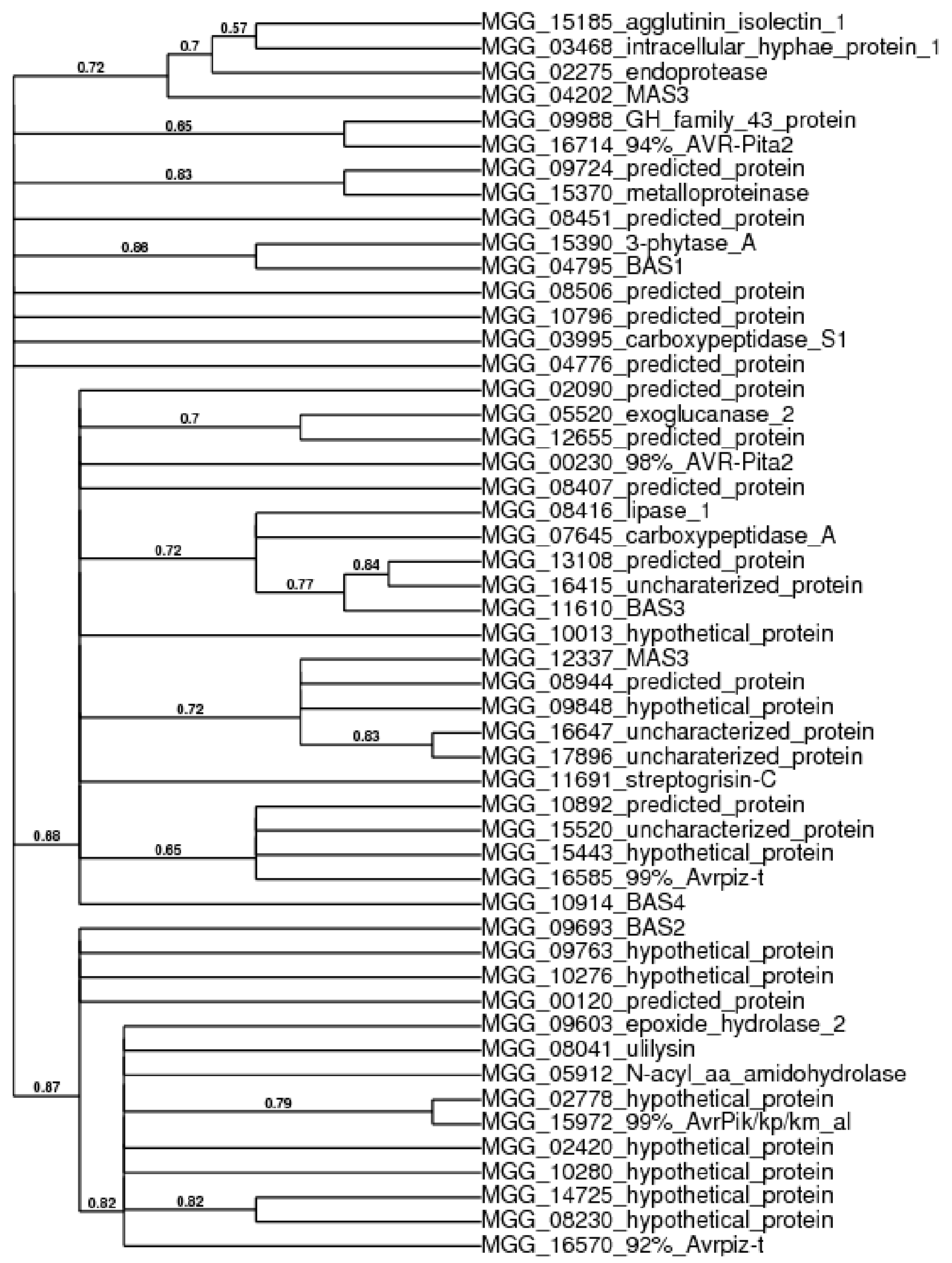

Figure 3.6: Maximum Likelihood based phylogenetic relationship between protein sequences of the top 50 putative secreted proteins induced in planta. Multiple sequence alignment was performed using MUSCLE (v3.7) with default settings, and edition of the phylogenetic tree were performed with TreeDyn (v198.3). The analysis was performed on the Phylogeny.fr platform (Dereeper et al. 2008). The putative secretome formed three major clusters. Branch support values calculated in the bootstrap test (1000 replicates) are shown on the branches. 


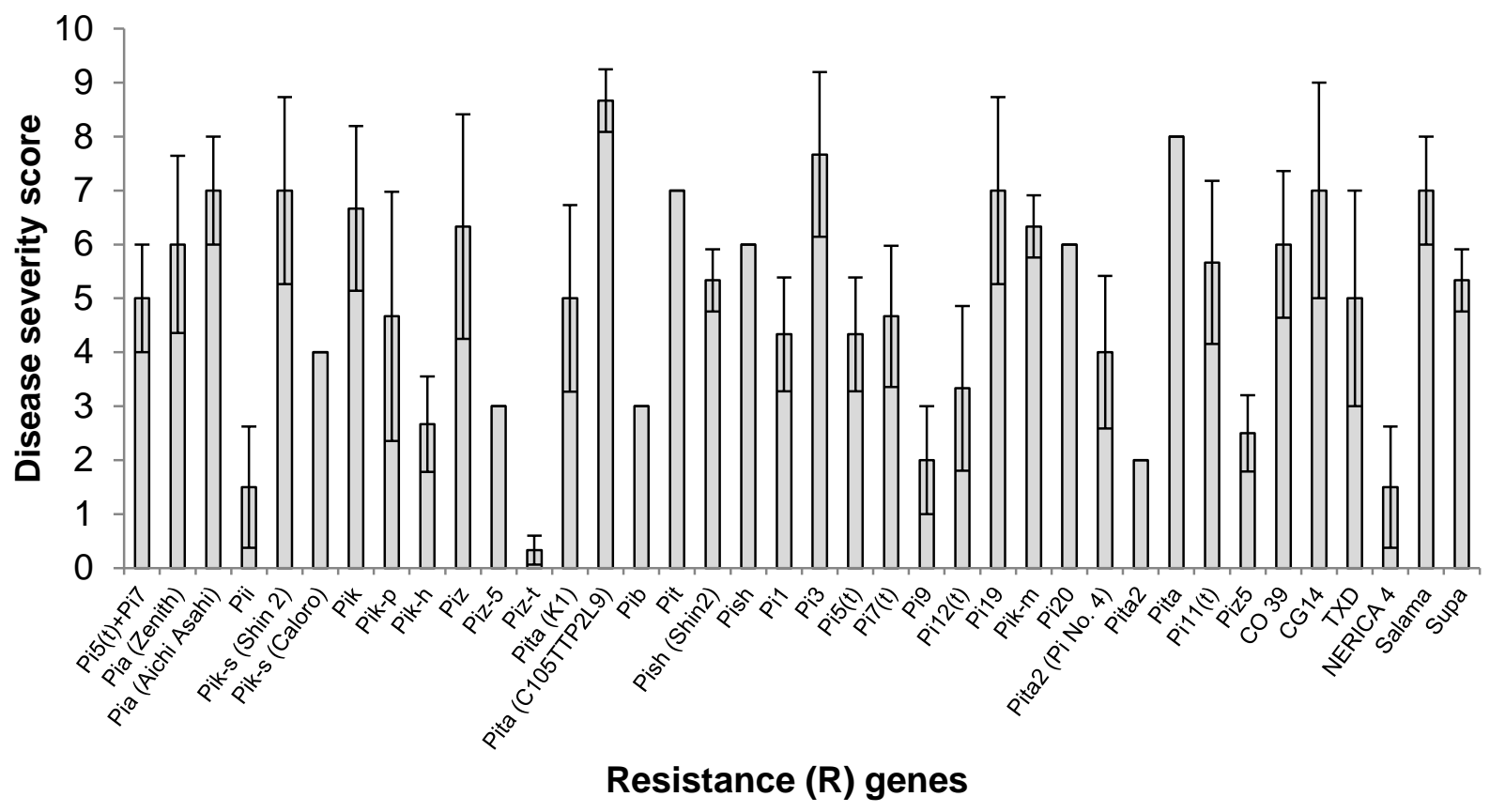

Figure 3.7: Reaction of 32 rice differential lines to TAN211.16. Piz-t was highly resistant to TAN211.16. Data are presented as mean disease severity scores \pm standard deviation in 3 replicates.

A

Motif 1

1.0e-020 KNMVEFR YL GTMDMALLRGYAETCLA I VYFAKTSDDFKYAALLDE I CEC 2 sites

Motif 2

3.5e-020 V DDFLWF I DLA LG A RTGG WT IKVTCRLC I GDRVSDYEE CR

Motif 3

2.3e-019 RT V RNATTSFWV LS I GSDVWE TVFRRLYMLM LVE TVCSMFV SW 2 sites

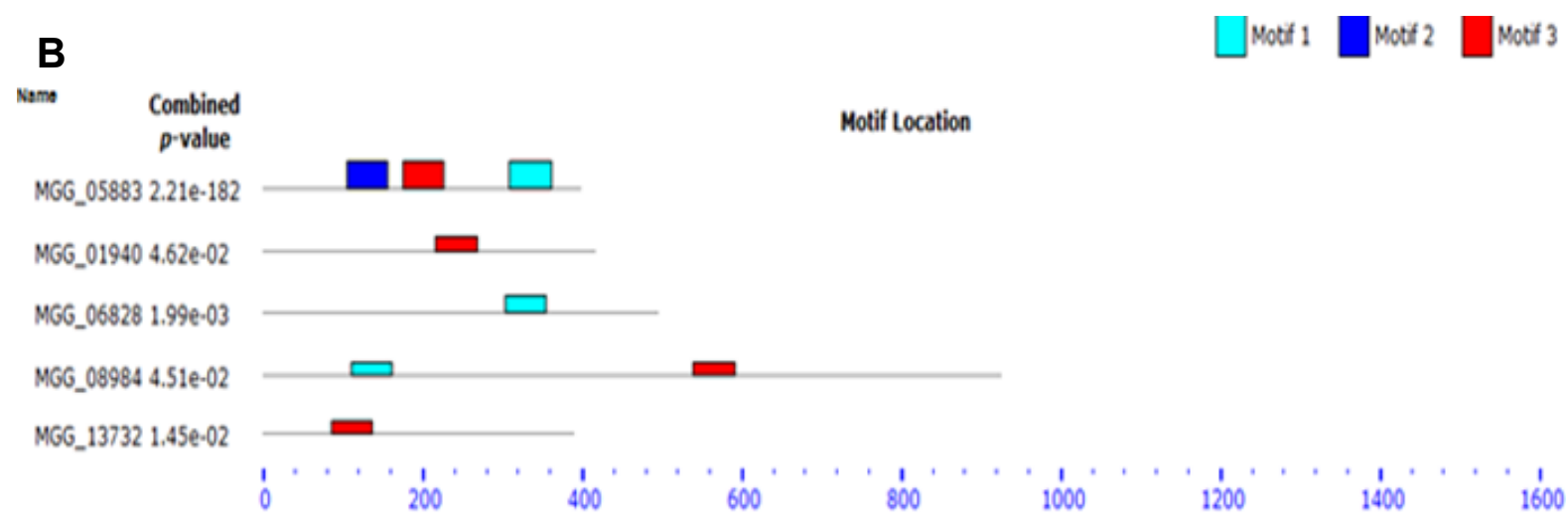

Figure 3.8: MEME searching identified overrepresented motifs in Specific genes up-regulated in NIp35i. Sequence logo of the top scoring MEME result for the top 11 regulated genes by SAM score (A) ; and (B) position and scoring of motif sites ( $\mathrm{p}$-value threshold $<5 \mathrm{e}-2)$ in upstream sequences. The motifs were present in the upstream sequences of 10 genes often with multiple instances, as shown by the color blocks depicting motif position within upstream sequences. Only 5 significant genes $(>0.05)$ are presented. 
Chapter 3: Transcript profiles of Magnaporthe oryzae in planta expressed genes and rice defence response genes in plants pre-exposed to high temperature

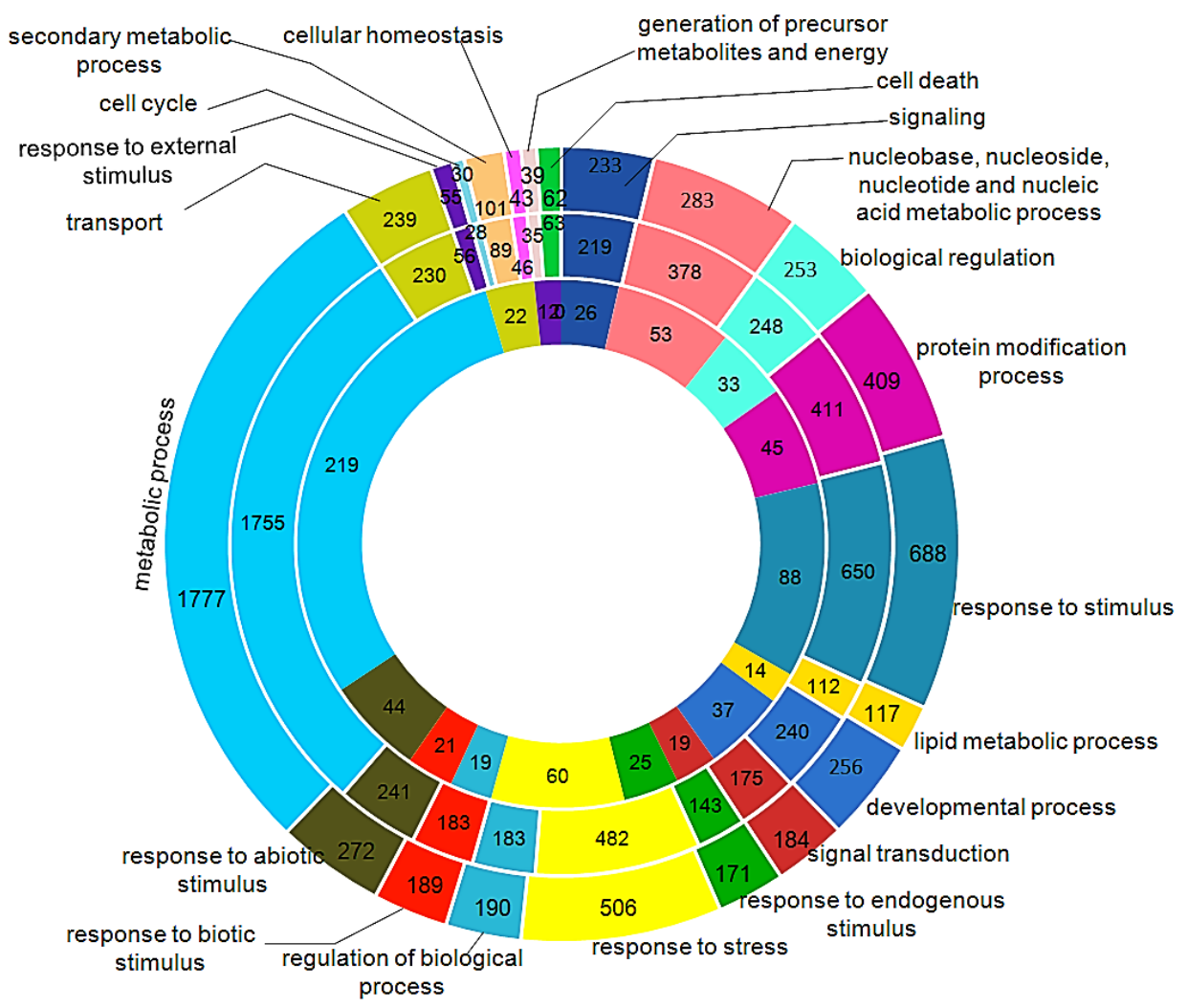

Figure 3.9: Gene Ontology classification of up regulated rice DEGs in Nip35c (inner circle), Nip35i (middle circle) and Nip28i (outer circle), categorized into 23 biological processes. The number of genes enriched in a given biological process is presented in the segments of each circle.

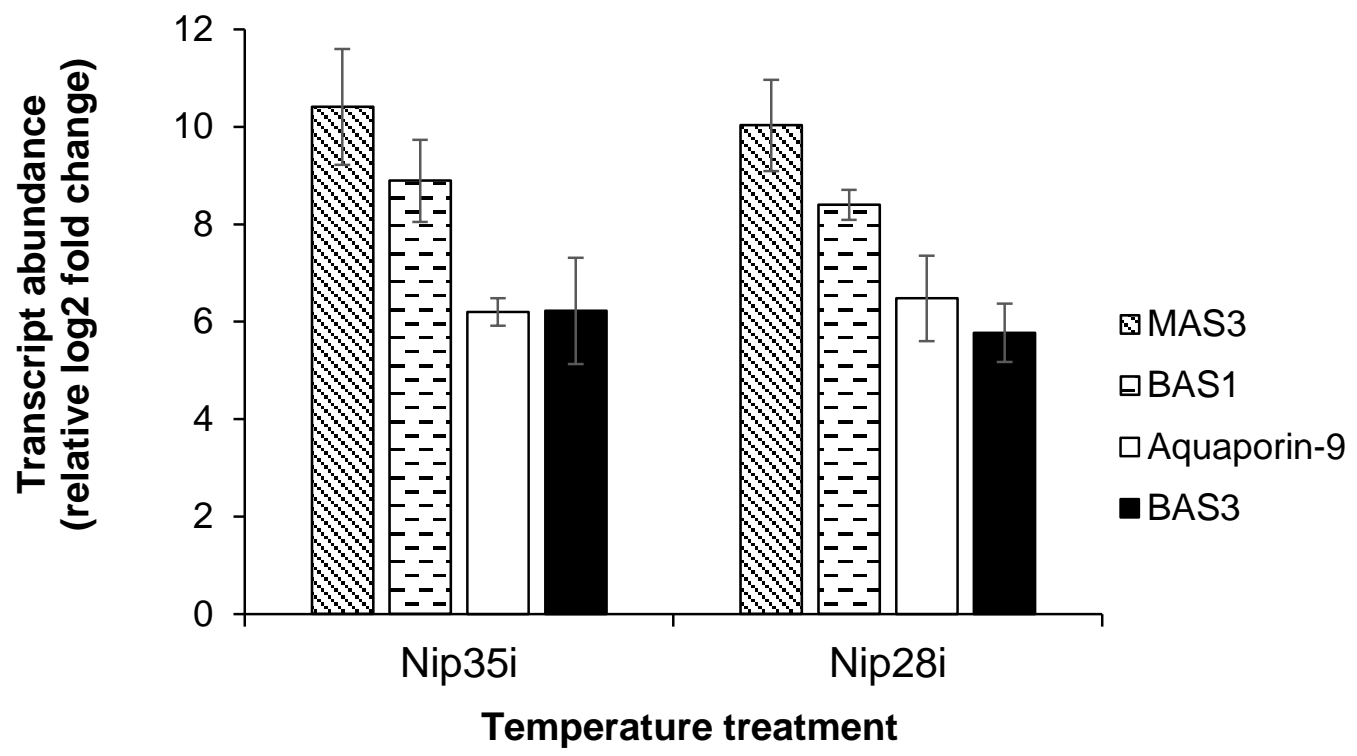

Figure 3.10: qPCR analysis of MAS3, BAS1, aquaporin-9 and BAS3 expression in infected rice leaves exposed to high temperature (Nip35i) and normal temperature (Nip28i). The relative gene transcripts were calculated relative to the fungal control using Pffal method, values are means \pm SD. 


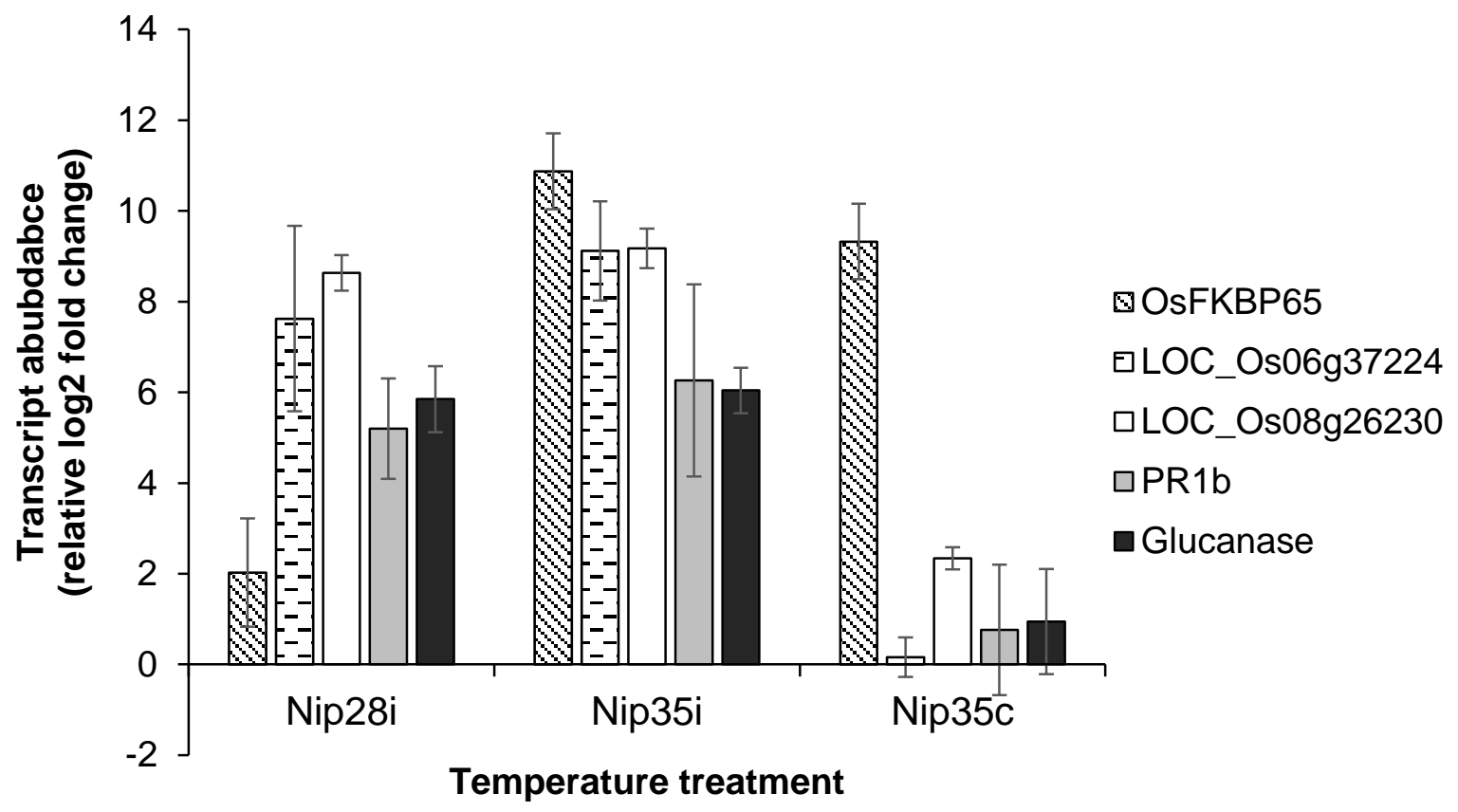

Figure 3.11: qPCR analysis of OsFKBP65, expressed protein (LOC_Os06g37224), Cytochrome P450 (LOC_Os08g26230), PR1b and PAL expression in infected rice leaves exposed to high temperature (Nip35i), normal temperature (Nip28i) and only high temperature without M. oryzae infection. The relative gene transcripts were calculated relative to the control (Nip28c) using Pffal method. Values are means \pm SD. 


\title{
Chapter 4: Population structure, pathogenicity and mating type distribution of Magnaporthe
}

\author{
oryzae isolates from East Africa
}

Geoffrey Onaga, Kerstin Wydra, Birger Koopmann, Yakouba Séré, and Andreas von Tiedemann

First, third, and fifth authors: Division of Plant Pathology and Crop Protection, Department of Crop Sciences, and second author: CLB-Tropical and Subtropical Agriculture and Forestry, Georg-August-University Göttingen, Germany; and fourth author: Africa Rice Center, P.O. Box 33581, Dar es Salaam, Tanzania.

Accepted for publication 23 March 2015.

\section{ABSTRACT}

Onaga, G., Wydra, K., Koopmann, B., Séré, Y., and von Tiedemann, A. 2015. Population structure, pathogenicity, and mating type distribution of Magnaporthe oryzae isolates from East Africa. Phytopathology 105:1137-1145.

Rice blast, caused by Magnaporthe oryzae, is one of the emergent threats to rice production in East Africa (EA), where little is known about the population genetics and pathogenicity of this pathogen. We investigated the genetic diversity and mating type (MAT) distribution of 88 isolates of $M$. oryzae from EA and representative isolates from West Africa (WA) and the Philippines (Asia) using amplified fragment length polymorphism markers and mating-type-specific primer sets. In addition, the aggressiveness of each isolate was evaluated by inoculating on the susceptible Oryza sativa indica 'Co39', scoring the disease severity and calculating the disease progress. Hierarchical analysis of molecular variance revealed a low level of genetic differentiation at two levels $\left(F_{S T}\right.$ 0.12 and $\mathrm{F}_{\mathrm{CT}} 0.11$ ). No evidence of population structure was found among the 65 isolates from EA, and gene flow among EA populations was high. Moreover, pairwise population differentiation $\left(G_{S T}\right)$ in EA populations ranged from 0.03 to 0.04 , suggesting that $>96 \%$ of genetic variation is

Rice is a staple food for more than 3 billion people worldwide, and a significant income source to rural communities in developing countries. In East Africa (EA), rice has gained importance only recently. The production area in the three EA countries-Uganda, Rwanda, and Tanzania-increased from approximately 452,000 ha in 1999 to $1,223,910$ ha in 2011 (FAOSTAT 2011). This area expansion and the ongoing intensification of rice production could potentially contribute to increased rice supply in the region that was formerly dependent on maize and sorghum as major cereals. However, rice production in EA faces a double burden of destructive diseases and the knowledge gap to cope with disease outbreaks. Among the various diseases, rice blast caused by Magnaporthe oryzae is an eminent threat to rice production. In fact, an unexpected blast outbreak was observed in farmers' fields in Kenya in 2009, leading to $48 \%$ yield losses (Kihoro et al. 2013). Moreover, field observations in Tanzania, Burundi, and Rwanda indicate the spread of the disease from traditional to new rice-growing areas, a situation that is only anticipated to worsen with increasing temperatures due to climate change (Liu et al. 2010; Mwalyego et al. 2011). How far this spread of the disease in EA indicates the evolution of novel pathotypes and enhancement of population diversity is not known.

Previous studies of genetic variation in $M$. oryzae populations found higher levels of genetic variation in field populations collected from South, East, and Southeast Asia than in other

Corresponding author: G. Onaga; E-mail address: gonaga@gwdg.de

http://dx.doi.org/10.1094/PHYTO-10-14-0281-R

(C) 2015 The American Phytopathological Society derived from within populations. However, the populations from Asia and WA were moderately differentiated from EA ones. The spatial analysis of principal coordinates and STRUCTURE revealed overlapping between individual $M$. oryzae isolates from EA, with limited distinctness according to the geographic origin. All the populations were clonal, given the positive and significant index of association $\left(I_{A}\right)$ and standardized index of association $\left(r_{d}\right)$, which indicates a significant $(P<0.001)$ departure from panmixia $\left(I_{A}\right.$ and $\left.r_{d}=0\right)$. Both MAT1-1 and MAT1-2 were detected. However, MAT1-1 was more prevalent than MAT1-2. Pathogenicity analysis revealed variability in aggressiveness, suggesting a potential existence of different races. Our data suggest that either $M$. oryzae populations from EA could be distributed as a single genetic population or gene flow is exerting a significant influence, effectively swamping the action of selection. This is the first study of genetic differentiation of rice-infecting $M$. oryzae strains from EA, and may guide further studies on the pathogen as well as resistance breeding efforts.

Additional keywords: AFLP.

regions (Saleh et al. 2014; Zeigler 1998). However, fewer lineages were detected in the United States (Correll et al. 2009; Levy et al. 1991; Xia et al. 1993, 2000), Europe (Piotti et al. 2005; Roumen et al. 1997), Iran (Javan-Nikkah et al. 2004), Argentina (Consolo et al. 2008), Colombia (Levy et al. 1993; Zeigler 1998), Cuba (Fuentes et al. 2003), and West Africa (Takan et al. 2012), which is probably consistent with the hypothesis that asexual reproduction is dominant in the life cycle of $M$. oryzae. Commonly reported causes of genetic variability in Asian M. oryzae populations include high levels of genetic instability of traits such as pathogenicity, morphology, and fertility (Maciel et al. 2014), which could be congruent with the large number of pathotypes in some of the field populations reported.

However, there have been no studies on the genetic structure of the rice infecting $M$. oryzae field populations in EA. Improving our understanding of the genetic diversity, mating type (MAT) distribution, and aggressiveness of this pathogen is the most promising strategy that will provide a basis for implementation of various management approaches, including host plant resistance.

Various methods have been used to study the genetic diversity and population structure in M. oryzae, such as MGR 586 repetitive DNA sequence (Borromeo et al. 1993), Pot2 (Kachroo et al. 1994), randomly amplified polymorphic DNA (RAPD) (Sere et al. 2007), amplified fragment length polymorphism (AFLP) (Thuan et al. 2006), simple sequence repeats (SSR) (Brondani et al. 2000; Saleh et al. 2014), and pathogenicity tests on differential sets of rice genotypes (Levy et al. 1991). From these methods, AFLP and SSR provide more sensitive and reliable analysis for individual genotype profiling. The AFLP technique is particular suitable for genetic diversity analysis due to simultaneous analysis of a large number of 
polymorphic loci and high reproducibility due to high stringency of polymerase chain reaction (PCR), compared with RAPD (Liu and Cordes 2004). Due to these advantages, AFLP markers have been advocated as a powerful genetic marker system for assessing population structure and individual identity (Gerber et al. 2000).

Our objective here was to assess the extent of genetic variation in EA field populations of $M$. oryzae with AFLP markers, and determine MAT distribution and virulence. The present study provides the first estimates of genetic structure of field populations of M. oryzae from EA.

\section{MATERIALS AND METHODS}

Sampling, monoconidial isolation, and cultivation of fungal isolates. Isolates were collected in 2009 and 2011 from three EA countries: Rwanda, Uganda, and Tanzania (Fig. 1). For comparative purposes, isolates from West Africa and the Philippines (Asia) were included. Eight sampling points (farmers' fields) per site were considered. One to nine cultivars were sampled per location, with one to five lesion-bearing leaves collected per cultivar and one isolate collected per leaf. The number of sampling points and sites sometimes varied, according to the rice field and disease availability. Prior to isolation, each leaf sample was placed on glass rods in petri dishes with wet filter papers and incubated in continuous fluorescent light for 1 to 2 days at $25^{\circ} \mathrm{C}$ until conidiophores bearing conidia emerged from stromatal tissue beneath leaf stomata. The sporulating lesions were examined under a stereomicroscope and a group of conidia was aseptically transferred with a transfer needle to V8 agar. The pathogen was identified based on its morphological growth pattern and spore shape. To obtain single conidial isolates, conidia were streaked onto water agar. Individual, germinating conidia were identified with

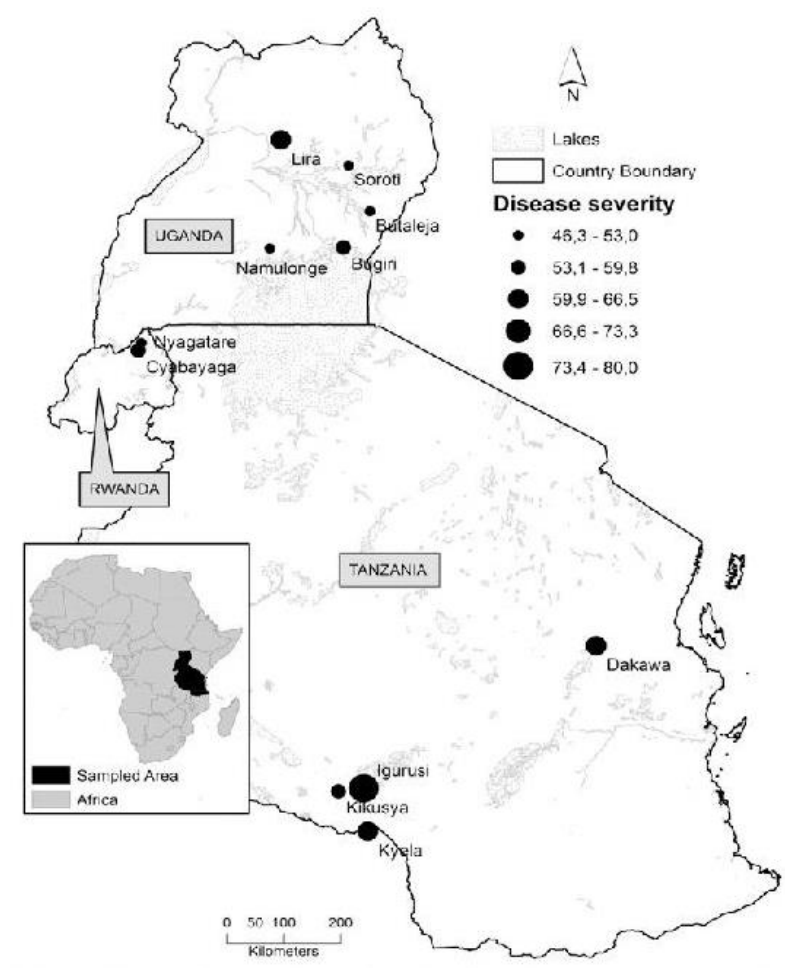

Fig. 1. Geographic sites of rice fields in East Africa where Magnaporthe oryzae strains were sampled. Mean percent disease severity scores (black dots) for the different sampling sites and respective locations are indicated. The size of the dots indicates the level of disease severity. a stereomicroscope and aseptically transferred and cultured in V8 agar. For long-term storage, each culture was overlaid with several sterilized filter paper sections and incubated at $25^{\circ} \mathrm{C}$. After 10 to 12 days of incubation, the colonized filter paper sections were lifted from the agar surface, placed in sterile, empty petri dishes, allowed to dry for 3 days at room temperature, and stored at $-20^{\circ} \mathrm{C}$.

DNA extraction. In total, 88 isolates of $M$. grisea (Table 1) from the collection stored in the Division of Plant Pathology and Crop Protection, Georg-August University, Goettingen, Germany, were used for AFLP analysis. To obtain mycelia, plugs harvested from 8-day-old cultures were inoculated into a 1,000-ml flask containing $250 \mathrm{ml}$ of liquid Fries medium at room temperature for 7 to 8 days with constant shaking on a rotary shaker. Mycelial mats were harvested, freeze dried, and ground in liquid nitrogen to a fine powder. Approximately $40 \mathrm{mg}$ of the powdered mycelia was used for DNA isolation using a modification of the cetyltrimethylammonium bromide (CTAB) method (Brandfass and Karlovsky 2008), by combining the sodium acetate-sodium dodecyl sulfate with CTAB extraction.

AFLP analysis. AFLP analysis was conducted according to a method modified from Vos et al. (1995). The primary template for preamplification reaction consisted of $125 \mathrm{ng}$ of genomic DNA. Genomic DNA was first digested with $10 \mathrm{U}$ of EcoRI at $37^{\circ} \mathrm{C}$ overnight followed by a second digestion with $5 \mathrm{U}$ of $T r u \mathrm{I}$ (Fermentas $\mathrm{GmbH}$, St. Leon-Rot, Germany) at $65^{\circ} \mathrm{C}$ for $2 \mathrm{~h}$. The digested samples were ligated overnight with $1.0 \mathrm{U}$ of T4 DNA ligase (Fermentas $\mathrm{GmbH}$ ) and $10 \mu \mathrm{M} \mathrm{EcoRI}$ and $T r u \mathrm{I}$ adapters in a final volume of $20 \mu \mathrm{l}$ at room temperature. The ligation products were diluted 10 -fold with ultrapure water and $1 \mu \mathrm{l}$ of the resulting dilution was used for preselective amplification with EcoRI-A and TruI-C primers $(10 \mu \mathrm{M}), 10 \mathrm{mM}$ each dNTP, $50 \mathrm{mM} \mathrm{MgCl}_{2}, 5 \mathrm{U}$ of Taq DNA polymerase, and $10 \times$ Taq polymerase buffer $(50 \mathrm{mM}$ Tris$\mathrm{HCl}$ [pH 7.5], $50 \mathrm{mM}$ magnesium acetate, and $250 \mathrm{mM}$ potassium acetate) in a total volume of $25 \mu$. Two replicate AFLP reactions per individual were used to eliminate variation due to anomalous PCR products or artifacts of detection in individual analysis. PCR was performed for 25 cycles, which consisted of $30 \mathrm{~s}$ at $94^{\circ} \mathrm{C}, 1 \mathrm{~min}$ at $56^{\circ} \mathrm{C}$, and $1 \mathrm{~min}$ at $72^{\circ} \mathrm{C}$ in an Eppendorf Master Cycler (NethelerHinz GmbH, Hamburg, Germany). The products of the preselective amplification step were diluted 1:10 with ultrapure water and subjected to selective amplification. The amplification reaction mix was similar to the preselective amplification, except that labeled EcoRI-ACA-Dy635-5 carboxyfluorescein, EcoRI-ACC-Dy750 blue fluorescein, EcoRI-AGA-Dy680 green fluorescein (Dyomics, Jena, Germany), and TruI-CCC primers were used. The thermocycler program consisted of two phases. The first phase was performed with a touchdown cycling profile of 12 cycles with a step-down of annealing temperature by $0.7^{\circ} \mathrm{C} /$ cycle: $30 \mathrm{~s}$ at $94^{\circ} \mathrm{C}$, $30 \mathrm{~s}$ at 65 to $57.3^{\circ} \mathrm{C}$, and $1 \mathrm{~min}$ at $72^{\circ} \mathrm{C}$. The second phase consisted of 23 cycles of $30 \mathrm{~s}$ at $94^{\circ} \mathrm{C}, 1 \mathrm{~min}$ at $56^{\circ} \mathrm{C}$, and $1 \mathrm{~min}$ at $72^{\circ} \mathrm{C}$

Preparation of DNA amplification fragments for separation by capillary electrophoresis. The selectively amplified DNA fragments were diluted 1:10 with ultrapure water, and $2.5 \mu \mathrm{l}$ of each sample was loaded into respective microtiter plate wells. A 30- $\mu 1$ separation solution (formamide incorporated) mixed with a size standard 600 (Beckman Coulter, Fullerton, CA) was added and each well was overlaid with a drop of Sigma mineral oil (M5904). The microtiter plates containing the reaction mixtures were briefly spun and the samples were run in denaturing polyacrylamide gel using an automated DNA sequencer (CEQ 8000 genetic analysis system; Beckman Coulter). The AFLP patterns were captured and peaks were assigned to base pair sizes using the default analysis parameter and analyze the data window in the CEQ System Software. Todetect peaks of interest, the slope threshold and the relative peak height threshold were set at 50 and 10 , respectively. All peak heights were compared with a reference peak of the same color based on the migration time for each of the dyes used. Further data processing, including background subtraction and signal matching, was 
performed according to Weiberg and Karlovsky (2009). The peak data were converted into a binary matrix using the AFLP feature of the CEQ System. The AFLP feature creates bins from fragments with sizes that fall within a user-defined bin width. For each of the possible fragments, a " 1 " is assigned if a fragment is found in that sample and " 0 " if the fragment is absent. A table of $1 \mathrm{~s}$ and $0 \mathrm{~s}$ is created and exported to an excel spreadsheet. Only PCR fragments that show high reproducibility in the two repeat runs were used for further analyses.

Population genetic structure analysis. The genetic diversity within and between populations was assessed with POPGENE, version 1.3.2 (Yeh and Boyle 1999). Shannon's index $(I)$ and Nei's diversity index $(H)$ were also calculated using the default settings in POPGENE. The genetic differentiation $\left(G_{S T}\right)$ among populations was calculated as $G_{S T}=D_{S T} / H t$. Gene flow $(\mathrm{Nm})$ between any two studied populations was estimated as $N m=0.5\left(1-G_{S T}\right) / G_{S T}$. The average allele frequency of alleles unique to one population, across loci $(p[1])$, or private alleles was estimated using GENALEX. We used the poppr $\mathrm{R}$ package to generate a Maynard Smith index of association $\left(I_{A}\right)$, which assesses multilocus linkage disequilibrium, and another estimator, $r_{d}$, to correct for the bias in $I_{A}$ that may arise due to the influence of increasing number of loci. We estimated multilocus genotypic diversity using Stoddart and Taylor's index $G$ (Stoddart and Taylor 1988), and multilocus genotypic evenness (the distribution of genotypes in a sample) using the index $E_{5}$ (Grünwald et al. 2003). Two methods were used to determine population genetic structure. First, the program Tools for Population Genetic Analysis (TFPGA) (Miller 1997) was used to calculate $\theta$, an unbiased estimator of Wright's $\mathrm{F}_{\mathrm{st}}$. We calculated $95 \%$ confidence intervals around the estimates of $\theta$ by bootstrapping with 1,000 replicates. Second, the population genetic analysis program ARLEQUIN 3.5 (Excoffier et al. 2005) was used to assess the interpopulation genetic structure without assuming Hardy-Weinberg equilibrium by performing an analysis of molecular variance (AMOVA).

Cluster analysis of AFLP data. Cluster analysis of the presence (1) or absence (0) data matrix was performed with two methods. We used principle coordinate analysis (PCoA) based on Nei's distances between all pairs of AFLP genotypes using GENALEX 6.2 (Peakall and Smouse 2006; Nei 1978), which generated a two-dimensional principal component analysis plot showing the distribution of $M$. oryzae strains among the studied populations. Second, we used the model-based program STRUCTURE 2.3 (Pritchard et al. 2000), which implements a clustering algorithm based on a Bayesian model to assign individuals to a number of clusters $(\mathrm{K})$ or populations. To estimate the number of $\mathrm{Ks}, 10$ independent runs were performed for each number of possible clusters $(K=1$ to 10$)$, without any prior information on the origin of individual isolates. For each run, we used a burn-in period of $1 \times 10^{5}$ iterations followed by a run length of $1 \times 10^{5}$ iterations, using a model with correlated allele frequencies and admixture among populations. The number of populations that best represent the observed data under the model implemented was determined using STRUCTURE output, based on the rate of change in log probability of data $(\mathrm{LnP}[\mathrm{D}])$ between successive Ks. The most probable value of $\mathrm{K}$ was determined by $\Delta \mathrm{K}$, an ad hoc quantity related to the second-order change in the $\mathrm{Ln} P(\mathrm{D})$ with respect to the number of clusters inferred by STRUCTURE (Evanno et al. 2005). The estimated membership coefficients for each individual in each cluster in a Q matrix were passed as input to the software package DESTRUCT for visualization.

Pathogenicity assays and statistical analysis. Plant inoculations were performed on short-duration dwarf Oryza sativa indica 'Co39', with high susceptibility to tropical blast isolates. The plants were grown in the greenhouse at $25 \pm 5^{\circ} \mathrm{C}$ with a photoperiod of $14 \mathrm{~h}$ for 23 days. $M$. oryzae isolates were grown in petri dishes containing V8 agar under $14 \mathrm{~h}$ of fluorescent light at $25^{\circ} \mathrm{C}$ for 12 days. Three days prior to inoculation, cultures were exposed to low levels of visible and near-UV radiation to maximize sporulation.
Conidia were harvested with sterile distilled water and the concentration was adjusted to $1 \times 10^{5}$ conidia/ml after filtration through two layers of Miracloth. Pathogenicity tests were performed by spraying a conidial suspension containing $0.05 \%$ Tween 20 onto rice seedlings at four- to five-leaf stages. Inoculated plants were kept in a humidified growth chamber in the dark at $25^{\circ} \mathrm{C}$ for $24 \mathrm{~h}$, before returning to the greenhouse at $25 \pm 5^{\circ} \mathrm{C}$. Leaf blast was assessed 4 to 12 days after inoculation using a disease evaluation scale from 0 to 9 (Silva et al. 2009). Inoculations were repeated two times using three replicates per repeat to confirm lesion categorization into resistant or susceptible types. Disease severity ratings between the two repeats remained consistent. Violation of assumptions of normality was tested on both datasets using the Shapiro-Wilks test, and the data set with minimal bias in the distribution of residuals was used for calculating the area under the disease progress curve (AUDPC) using the midpoint rule method (Campbell and Madden 1990) in order to compare the different isolates. Analysis of variance for the final rice blast severity (\%) and AUDPC was performed using SAS 9.3, and the least significant difference test at 0.05 probability level was used for mean comparisons.

MAT distribution. Two isolates, IN1 (MAT1-1) and OG5 (MAT1-2), kindly provided by Dr. Didier Tharreau (CIRAD, Montpellier, France), were used as testers in this study. DNA of the testers was isolated as described above. Isolates used for MAT determination are indicated in Table 1. Two singleplex PCR amplifications were performed with MAT-specific primers previously used by Consolo et al. (2005): A1 (5'-AGCCTCATCAACGGCAA-3') andA5 (5'-GGCACGAACATGCGATG-3') for MAT1-1 and B15 $\left(5^{\prime}\right.$-CTCAATCTCCGTAGTAG- $\left.3^{\prime}\right)$ and B16 (5'-ACAGCAGTA TAGCCTAC-3') for MAT1-2. The PCR had a final volume of $20 \mu \mathrm{l}$ containing $80 \mathrm{ng}$ of template DNA, 10× Taq polymerase buffer (Bioline, Luckenwalde, Germany), $2.5 \mathrm{mM}$ each dNTP, $50 \mathrm{mM}$ $\mathrm{MgCl}_{2}, \operatorname{TaqDNA}$ polymerase at $5 \mathrm{U} / \mu \mathrm{l}$, and $0.01 \mathrm{mM}$ each primer. Thermal cycling conditions included an initial denaturation step at $95^{\circ} \mathrm{C}$ for $5 \mathrm{~min}$ followed by 30 cycles of $95^{\circ} \mathrm{C}$ for $45 \mathrm{~s}, 58$ and $62.5^{\circ} \mathrm{C}$ for MAT1-2 and MAT1-1, respectively, for $1 \mathrm{~min}$, and $72^{\circ} \mathrm{C}$ for $1 \mathrm{~min}$; and a final elongation step at $72^{\circ} \mathrm{C}$ for $5 \mathrm{~min}$. PCR products were separated in a $1.2 \%$ agarose gel at $66 \mathrm{~V}$ for $3 \mathrm{~h}$, stained with ethidium bromide, and photographed.

\section{RESULTS}

Genetic diversity and population structure. The three selected AFLP primer combinations generated a total of $289 \mathrm{loci}$, of which 198 were phylogenetically informative; that is, present or absent in at least two strains, with alleles ranging in size from 60 to $700 \mathrm{bp}$ across all 88 isolates of the five populations. The percentage of polymorphic loci at the population level ranged from $31.3 \%$ (West Africa) to $68.7 \%$ (Uganda) (Table 2). To distinguish clonal and recombinant structures in all populations, multilocus genotypes (MLG) and $I_{A}$ were used. We detected clonality in all the populations, given the positive $I_{A}$ and $r_{d}$ values (Fig. 2), which indicate a significant $(P<0.001)$ departure from panmixia $\left(I_{A}\right.$ and $\left.r_{d}=0\right)$. The frequency of MLG in each population was significantly correlated with AFLP loci obtained, indicating widespread linkage disequilibrium. The average gene diversity, Shannon's index, and evenness were estimated to be $0.13,0.24$, and 1 , respectively. Private alleles were found in all populations; the highest number was observed in Asia, followed by Uganda.

All populations showed low values of genetic differentiation $\left(G_{S T}\right)$, with an overall average among populations of 0.11 (Table 3 ). Low interpopulation genetic differentiation $(\theta=0.078,95 \%$ confidence interval $=0.057$ to 0.099 ) was equally found when the data were analyzed with TFPGA. Moreover, AMOVA, at two hierarchical levels $\left(\mathrm{F}_{\mathrm{ST}} 0.12\right.$ and $\left.\mathrm{F}_{\mathrm{CT}} 0.11\right)$, indicated that the populations were not geographically structured. Thus, most of the genetic variation was distributed within populations $(88.09 \%)$ 
( $P<0.001,1,023$ nonparametric permutations) rather than among groups (Asia, West Africa, and EA) (10.97\%), and no significant variation existed between populations within groups $(0.93 \%)$ (Table 4). Correspondingly, gene flow between populations was high (>1). Gene flow was highest between Uganda and Rwanda $(\mathrm{Nm}=16.78)$, Tanzania and Uganda $(\mathrm{Nm}=13.10)$, and Tanzania and Rwanda $(\mathrm{Nm}=10.84)$, and was consistent with $I_{A}$ and population evenness. Tanzania, Uganda, and Rwanda also recorded the highest indices of genetic identity and the lowest levels of genetic distance, corroborating the data obtained for gene flow and genetic differentiation. Conversely, gene flow between Asian and African populations was relatively low.

Cluster analysis. PCoA, based on Nei's genetic distance, grouped the isolates into four main clusters (Fig. 3A). The PCoA captured $54.1 \%$ of the total variation in two principal components. The first principal component accounted for $35.7 \%$ of the total variation and illustrated the outgroup position of the Philippine isolates (group 1). Group 2 constituted the largest admixed group followed by group 3 . Isolates from the various collection sites formed 15 subpopulations (Fig. 3B). Tanzanian subpopulations were closer to the West African subpopulation, whereas Rwanda and Uganda were found closer to each other. Using STRUCTURE 2.3, Ln probabilities $(\mathrm{X} \mid \mathrm{K})$ for $\mathrm{K}$ values (number of assumed populations) of 1 to 10 were calculated, and $\mathrm{K}=5$ had the highest probability (Fig. 4A). Thus, five genetic groups were resolved (Fig. 4B). The results showed a clear separation of the Philippine isolates from the African groups. Among the African populations, West African isolates were the genetically most homogeneous, which was consistent with the 0 private alleles and the high $I_{A}$ values observed (Table 2). Populations from Uganda, Tanzania, and Rwanda had a large proportion of admixed individuals, which corroborates the trend observed in the PCoA clustering. Interestingly, we found that variation in AFLP genotypes was not predictive (not correlated) of variation in pathogenicity.

Pathogenicity assays. Isolates varied in pathogenicity, as reflected by the percent leaf area infected (Table 1). At 10 days postinoculation (dpi), $76 \%$ of isolates hadinduced severity scores of $\geq 50 \%$. The remaining $24 \%$ consistently produced restricted lesion sizes. The most virulent isolates were TAN211.8 and 507RWA11 from Tanzania and Rwanda, respectively. The least virulent isolate

TABLE 1. Code, designation, country of origin, site of collection, year of collection, cultivars from which the strains were isolated, mating type, and area under the disease progress curve (AUDPC) obtained from pathogenicity tests

\begin{tabular}{|c|c|c|c|c|c|c|c|}
\hline Number & Code & Designation & Country & Site of collection & Cultivar & Mating type & AUDPC ${ }^{z}$ \\
\hline 1 & 1026 & 501UGA09 & Uganda & Tilda, Bugiri & Pusa Basmati & MAT1-2 & $495.0 \mathrm{de}$ \\
\hline 2 & 1027 & 502UGA09 & Uganda & Tilda, Bugiri & Pusa Basmati & MAT1-2 & 480.0 def \\
\hline 3 & 1028 & 503UGA09 & Uganda & Tilda, Bugiri & Pusa Basmati & MAT1-2 & $215.0(2) \mathrm{i}-1$ \\
\hline 4 & 1029 & 504UGA09 & Uganda & Tilda, Bugiri & Pusa Basmati & MAT1-2 & $\ldots$ \\
\hline 5 & 1030 & 505UGA09 & Uganda & Tilda, Bugiri & Pusa Basmati & MAT1-2 & $452.5 \mathrm{fgh}$ \\
\hline 6 & 1031 & 508UGA09 & Uganda & Tilda, Bugiri & Pusa Basmati & MAT1-1 & $235.0(2) \mathrm{f}-\mathrm{k}$ \\
\hline 7 & 1032 & 513UGA09 & Uganda & Tilda, Bugiri & Pusa Basmati & MAT1-1 & $470.0 \mathrm{~d}-\mathrm{g}$ \\
\hline 8 & 1033 & 518UGA09 & Uganda & Tilda, Bugiri & Pusa Basmati & MAT1-2 & $225.0(2) \mathrm{h}-\mathrm{k}$ \\
\hline 9 & 1035 & 520UGA09 & Uganda & Tilda, Bugiri & Pusa Basmati & MAT1-2 & $327.5 \mathrm{~s}-\mathrm{W}$ \\
\hline 10 & 1037 & 524UGA09 & Uganda & Tilda, Bugiri & Pusa Basmati & MAT1-2 & $330.0 \mathrm{r}-\mathrm{v}$ \\
\hline 11 & 1106 & 531 UGA11 & Uganda & Lira Itek Main Dam & Unknown & MAT1-1 & $510.0 \mathrm{~cd}$ \\
\hline 12 & 1108 & 533UGA11 & Uganda & Lira Itek Main Dam & Unknown & MAT1-1 & $155.0(2) \mathrm{nm}$ \\
\hline 13 & 1112 & 537UGA11 & Uganda & Soroti-Apalitunga & Unknown & MAT1-1 & $267.5(2) a-g$ \\
\hline 14 & 1113 & 538UGA11 & Uganda & Soroti-Apalitunga & Unknown & MAT1-1 & 282.5 yz(2)a-e \\
\hline 15 & 1114 & 539UGA11 & Uganda & Lira-Abolet & Unknown & MAT1-1 & $330.0 \mathrm{r}-\mathrm{v}$ \\
\hline 16 & 1116 & 541UGA11 & Uganda & Lira-Abolet & Unknown & MAT1-1 & $250.0(2) \mathrm{c}-\mathrm{i}$ \\
\hline 17 & 1117 & 542 UGA 11 & Uganda & Namulonge & NERICA 4 & MAT1-2 & $387.5 \mathrm{k}-\mathrm{p}$ \\
\hline 18 & 1121 & 547UGA11 & Uganda & Namulonge & NERICA 2 & MAT1-1 & $325.0 \mathrm{~s}-\mathrm{x}$ \\
\hline 19 & 1122 & 548UGA11 & Uganda & Namulonge & NERICA 3 & MAT1-2 & $\ldots$ \\
\hline 20 & 1123 & 549UGA1 1 & Uganda & Namulonge & NERICA 4 & MAT1-2 & $332.5 \mathrm{r}-\mathrm{u}$ \\
\hline 21 & 1126 & 552UGA11 & Uganda & Butaleja, Doho Rice Scheme & K98 & MAT1-1 & $197.5(2) \mathrm{kl}$ \\
\hline 22 & 1129 & 556UGA11 & Uganda & Namulonge & Pakistan & MAT1-1 & $\ldots$ \\
\hline 23 & 1130 & 557UGA11 & Uganda & Namulonge & Unknown & MAT1-1 & $265.0(2) \mathrm{a}-\mathrm{h}$ \\
\hline 24 & 1132 & 559UGA11 & Uganda & Balita-Wakiso & NERICA 4 & MAT1-1 & $412.5 \mathrm{i}-\mathrm{m}$ \\
\hline 25 & 1142 & 563UGA11 & Uganda & Doho-Butaleja & K98 & MAT1-1 & $255.0(2) \mathrm{b}-\mathrm{i}$ \\
\hline 26 & 1111 & 536UGA11 & Uganda & & & & $370.0 \mathrm{n}-\mathrm{r}$ \\
\hline 27 & 1092 & 495UGA11 & Uganda & & & MAT1-1 & $245.0(2) \mathrm{e}-\mathrm{j}$ \\
\hline 28 & 1298 & Ugm4 & Uganda & Doho-Butaleja & K98 & MAT1-1 & $295.0 \mathrm{u}-\mathrm{z}(2) \mathrm{ab}$ \\
\hline 29 & 1299 & Lira 1-1 & Uganda & Lira Itek Main Dam & unknown & MAT1-1 & $482.5 \mathrm{def}$ \\
\hline 30 & 1300 & Ugm 1 & Uganda & Doho-Butaleja & K98 & MAT1-1 & $350.0 \mathrm{p}-\mathrm{t}$ \\
\hline 31 & 1301 & Ugm2 & Uganda & Doho-Butaleja & K98 & MAT1-1 & $\ldots$ \\
\hline 32 & 1019 & 461 RWA09 & Rwanda & Nyazatare experiment station & Nyiragakara & MAT1-1 & $\ldots$ \\
\hline 33 & 1021 & 470RWA09 & Rwanda & Nyazatare experiment station & Nyiragakara & MAT1-1 & $250.0(2) \mathrm{c}-\mathrm{i}$ \\
\hline 34 & 1022 & 474RWA09 & Rwanda & Nyazatare experiment station & Nyiragakara & MAT1-1 & $400.0 \mathrm{j}-\mathrm{m}$ \\
\hline 35 & 1023 & 482RWA09 & Rwanda & Nyazatare experiment station & Nyiragakara & MAT1-1 & $220.0(2) \mathrm{i}-1$ \\
\hline 36 & 1024 & 488RWA09 & Rwanda & Nyazatare experiment station & Nyiragakara & MAT1-1 & $437.5 \mathrm{~g}-\mathrm{j}$ \\
\hline 37 & 1025 & 492RWA09 & Rwanda & Nyazatare experiment station & Nyiragakara & MAT1-1 & $310.0 \mathrm{t}-\mathrm{z}$ \\
\hline 38 & 1090 & 493RWA11 & Rwanda & Cyabayaga & Kavamahanga & MAT1-1 & $370.0 \mathrm{n}-\mathrm{r}$ \\
\hline 39 & 1094 & 497RWA1 1 & Rwanda & Cyabayaga & Kavamahanga & MAT1-1 & $357.5 \mathrm{o}-\mathrm{s}$ \\
\hline 40 & 1095 & 498RWA1 1 & Rwanda & Cyabayaga & Nyiragakara & MAT1-2 & $285.0 x-z(2) a-e$ \\
\hline 41 & 1099 & 504RWA11 & Rwanda & Cyabayaga & Nyiragakara & MAT1-1 & $182.5(2) \mathrm{lm}$ \\
\hline 42 & 1133 & 505RWA1 1 & Rwanda & Cyabayaga & Yunikeng & MAT1-2 & $362.5 \mathrm{n}-\mathrm{s}$ \\
\hline 43 & 1147 & RWA 11.2 & Rwanda & Cyabayaga & Irrigated rice & MAT1-2 & $422.5 \mathrm{i}-1$ \\
\hline 44 & 1149 & 506RWA11 & Rwanda & Cyabayaga & Irrigated rice & MAT1-1 & $360.0 \mathrm{n}-\mathrm{s}$ \\
\hline 45 & 1150 & RWA 11.5 & Rwanda & Cyabayaga & Irrigated rice & MAT1-1 & $227.5(2) \mathrm{g}-\mathrm{k}$ \\
\hline 46 & 1151 & RWA 11.6 & Rwanda & Cyabayaga & Irrigated rice & MAT1-1 & $465.0 \mathrm{e}-\mathrm{h}$ \\
\hline \multirow[t]{2}{*}{47} & 1152 & 507RWA1 1 & Rwanda & Cyabayaga & Irrigated rice & MAT1-1 & $600.0 \mathrm{a}$ \\
\hline & & & & & & & (continued on next page) \\
\hline
\end{tabular}

\footnotetext{
${ }^{\mathrm{z}}$ Means followed by the same letter are not significantly different from each other.
} 
was 536UGA11 from Uganda, which showed only 30\% severity. Ten isolates did not produce any visible symptoms on rice seedlings. The same isolates had low sporulation ability when tested on V8 and oatmeal agar plates, and were not included in the second pathogenicity test. Disease progression varied widely. For all pathogenic isolates, a constant increase in the severity scores was observed from 4 to 10 dpi. By comparison, Tanzania and Rwanda had a larger proportion of the more aggressive isolates than Uganda.

MAT distribution. An assay of 88 isolates, based on MATspecific PCR primers, revealed the presence of both MAT1-1 and MAT1-2 in EA (Fig. 4; Table 1). In all, 65 isolates from the various collection sites were MAT1-1 and 17 isolates were MAT1-2. The distribution among isolates was 11, 4, and 2 of MAT1-2 type from Uganda, Rwanda, and Tanzania, respectively. Overall, the frequency of MAT1-1 was higher in EA and predominantly the only MAT in most sites. The representative strains from Asia and West Africa were predominantly MAT1-2.

\section{DISCUSSION}

We analyzed $M$. oryzae populations from EA and representative isolates from West Africa and the Philippines in attempts to infer genetic variability, MAT distribution, and strain pathogenicity. Our data showed low $G_{S T}$ and pairwise population differentiations among EA populations, suggesting a lack of population structure according to Nei's (1978) classification of population differentiation $\left(G_{S T}<0.05\right.$ is low, 0.05 to 0.15 medium, and $\left.>0.15 \mathrm{high}\right)$. The absence of a population structure often suggests low genetic diversity, largely due to genetically similar populations (Okori et al. 2004). Such populations are either of common origin or restricted distribution, reproduce exclusively asexually, or experience substantial gene flow coupled with genetic drift (e.g., due to limited host availability). However, in populations where mutation rates are high, it has been suggested that the $G_{S T}$ tends to fall back to zero as more novel alleles are added to a population. This happens because of the negative dependence of $G_{S T}$ on diversity (Jost 2008), and may occur in the case of $M$. oryzae, in which considerable genetic variation, even in the absence of sexual reproduction, has been reported (Couch et al. 2005). To circumvent the bias that may probably result due to mutation rates, we related $G_{S T}$ values to other genetic diversity indices. Our data revealed a $G_{S T}$ value of 0.04 (Table 3 ) among the EA populations, suggesting that $96 \%$ of genetic variation is derived from within populations. This is consistent with the low Shannon's indices $(0.10$ to 0.21 ) (Table 2$)$. The Shannon's index of 0.21 suggests that more than $78 \%$ of the genetic diversity between populations was accounted for by differences between individuals. This result is contrary to the findings of Kumar et al. (1999), where Shannon's diversity indices of 0.89 to 0.92 were found in the Himalayan highly diverse populations of $M$. oryzae. This might be because EA populations represent recent founder populations where only a few generations have passed since colonization.

We used $I_{A}$ to test the degree of nonrandom association between alleles at different loci (linkage disequilibrium). The $I_{A}$ calculated

TABLE 1. (continued from preceding page)

\begin{tabular}{|c|c|c|c|c|c|c|c|}
\hline Number & Code & Designation & Country & Site of collection & Cultivar & Mating type & AUDPC $\mathrm{z}$ \\
\hline 48 & 1156 & 508RWA11 & Rwanda & Cyabayaga & Irrigated rice & MAT1-1 & $207.5(2) \mathrm{j}-1$ \\
\hline 49 & 1158 & 510RWA11 & Rwanda & Cyabayaga & Irrigated rice & MAT1-1 & $\ldots$ \\
\hline 50 & 1096 & 500RWA11 & Rwanda & Cyabayaga & Irrigated rice & MAT1-2 & $290.0 \mathrm{v}-\mathrm{z}(2) \mathrm{abc}$ \\
\hline 51 & 1159 & 511RWA11 & Rwanda & Cyabayaga & Irrigated rice & MAT1-1 & $230.0(2) \mathrm{g}-\mathrm{k}$ \\
\hline 52 & 1163 & TAN211.1 & Tanzania & Dakawa & Supa & MAT1-1 & $350.0 \mathrm{p}-\mathrm{t}$ \\
\hline 53 & 1164 & TAN211.2 & Tanzania & Igurusi & SARO & MAT1-1 & $495.0 \mathrm{de}$ \\
\hline 54 & 1165 & TAN211.3 & Tanzania & Kikusya & Unknown & MAT1-1 & $\ldots$ \\
\hline 55 & 1166 & TAN211.4 & Tanzania & Kikusya & Unknown & MAT1-2 & $280.0 \mathrm{yz}(2) \mathrm{a}-\mathrm{e}$ \\
\hline 56 & 1167 & TAN211.5 & Tanzania & Kikusya & Unknown & MAT1-1 & 280.0 yz(2)a-e \\
\hline 57 & 1169 & TAN211.8 & Tanzania & Kikusya & Unknown & MAT1-2 & $577.5 \mathrm{ab}$ \\
\hline 58 & 1170 & TAN211.9 & Tanzania & Kikusya & Unknown & MAT1-1 & $380.0 \mathrm{~m}-\mathrm{q}$ \\
\hline 59 & 1171 & TAN211.10 & Tanzania & Kyela & V35 & MAT1-1 & $392.5 \mathrm{k}-\mathrm{o}$ \\
\hline 60 & 1172 & TAN211.11 & Tanzania & Kyela & V36 & MAT1-1 & $350.0 \mathrm{p}-\mathrm{t}$ \\
\hline 61 & 1173 & TAN211.12 & Tanzania & Kyela & V37 & MAT1-1 & \\
\hline 62 & 1174 & TAN211.13 & Tanzania & Kyela & V38 & MAT1-1 & $380.0 \mathrm{~m}-\mathrm{q}$ \\
\hline 63 & 1175 & TAN211.14 & Tanzania & Kyela & V39 & MAT1-1 & $380.0 \mathrm{~m}-\mathrm{q}$ \\
\hline 64 & 1176 & TAN211.15 & Tanzania & Kyela & V40 & MAT1-1 & $250.0(2) c-i$ \\
\hline 65 & 1178 & TAN211.17 & Tanzania & Kyela & V40 & MAT1-1 & $537.5 \mathrm{bc}$ \\
\hline 66 & 1180 & RBELOK-10-7-3 & Benin & Lokossa & Unknown & MAT1-2 & $335.0 \mathrm{r}-\mathrm{u}$ \\
\hline 67 & 1181 & RBELOK-10-11-2 & Benin & Lokossa & Unknown & MAT1-1 & $345.0 \mathrm{q}-\mathrm{t}$ \\
\hline 68 & 1182 & RBELOK-10-13-3 & Benin & Lokossa & Unknown & MAT1-2 & $245.0(2) \mathrm{e}-\mathrm{j}$ \\
\hline 69 & 1184 & RNIIBAD-09-5-1 & Nigeria & Ibadan & Unknown & MAT1-2 & $245.0(2) e-j$ \\
\hline 70 & 1185 & RNIIBAD-09-82-2 & Nigeria & Edozhigi & Unknown & MAT1-2 & $265.0(2) \mathrm{a}-\mathrm{h}$ \\
\hline 71 & 1187 & RNIIBAD-09-82-3 & Nigeria & Edozhigi & Unknown & MAT1-2 & $382.5 \mathrm{l}-\mathrm{p}$ \\
\hline 72 & 1183 & RBELOK-1022 & Benin & Lokossa & Unknown & MAT1-2 & $425.0 \mathrm{i}-\mathrm{k}$ \\
\hline 73 & 1179 & RBELOK-10-2-4 & Benin & Lokossa & Unknown & MAT1-1 & $447.5 \mathrm{f}-\mathrm{i}$ \\
\hline 74 & 955 & 43 & Philippines & IRRI & Unknown & MAT1-2 & $447.5 \mathrm{f}-\mathrm{i}$ \\
\hline 75 & 957 & AGT211 & Philippines & unknown & Unknown & MAT1-2 & $287.5 \mathrm{w}-\mathrm{z}(2) \mathrm{a}-\mathrm{d}$ \\
\hline 76 & 958 & V86014 & Philippines & IRRI & Unknown & MAT1-2 & $247.5(2) \mathrm{d}-\mathrm{j}$ \\
\hline 77 & 959 & B90289 & Philippines & IRRI & Unknown & MAT1-2 & $312.5 \mathrm{t}-\mathrm{z}$ \\
\hline 78 & 961 & M36-1-3-10-1 & Philippines & IRRI blast nursery & Unknown & MAT1-2 & $272.5 \mathrm{z}(2) \mathrm{a}-\mathrm{f}$ \\
\hline 79 & 963 & C9228-37 & Philippines & Caliraya, Laguna & Unknown & MAT1-2 & $370.0 \mathrm{n}-\mathrm{r}$ \\
\hline 80 & 977 & V86010 & Philippines & Bo.Bula, Camarines Sur & Unknown & MAT1-2 & $315.0 \mathrm{t}-\mathrm{y}$ \\
\hline 81 & 969 & M39-1-2-21-2 & Philippines & IRRI blast nursery & Unknown & MAT1-2 & $245.0(2) \mathrm{e}-\mathrm{j}$ \\
\hline 82 & 970 & M39-1-3-8-1 & Philippines & IRRI blast nursery & Unknown & MAT1-2 & 275.0 yz(2)a-f \\
\hline 83 & 973 & B 90002 & Philippines & Caliraya, Laguna & Unknown & MAT1-2 & $295.0 \mathrm{u}-\mathrm{z}(2) \mathrm{ab}$ \\
\hline 84 & 974 & M101-1-1-2-9-1 & Philippines & IRRI blast nursery & Unknown & MAT1-2 & $\ldots$ \\
\hline 85 & 976 & V850256 & Philippines & Cavinti, Laguna & Unknown & MAT1-2 & $310.0 \mathrm{t}-\mathrm{z}$ \\
\hline 86 & 983 & Ken53-33 & Japan & unknown & Unknown & MAT1-2 & $302.5 \mathrm{u}-\mathrm{z}(2) \mathrm{a}$ \\
\hline 87 & 952 & $\mathrm{Ca} 89$ & Philippines & Cavinti, Laguna & Unknown & MAT1-2 & $250.0(2) c-i$ \\
\hline 88 & 960 & V86046 & Philippines & Roxas, Palawan & Unknown & MAT1-2 & $\ldots$ \\
\hline
\end{tabular}


for all populations suggested clonality (Fig. 2), and was consistent with the population evenness and MLG. This indicates widespread linkage disequilibrium and suggests that $M$. oryzae populations from EA are limited to a small number of mostly closely related isolates. A similar pattern was observed with the West African reference collections. In the Asian collection, the number of private alleles was substantially larger, which indicates a greater proportion of diverse alleles compared with EA populations. The relatively short history of rice cultivation and the narrow genetic base of rice germplasm in EA compared with traditional rice-growing areas (e.g., South and Southeast Asia) may be thought of as a contributing factor. Indeed, intensive rice cultivation in EA occurred only recently, when Asian and West African developed varieties were introduced. These introduced cultivars could be causing minor shifts in $M$. oryzae populations already adapted, and may probably take some time for variations to cause a detectable differentiation. In contrast, populations of $M$. oryzae from South and Southeast Asia, where there is a high diversity of rice cultivars, are highly diverse, and clonality is not very obvious (Chen et al. 1995; Kumar et al. 1999; Park et al. 2003).

Exact tests of population differentiation also showed no evidence of differentiation between EA populations (data not shown), which

TABLE 2. Genetic diversity parameters for the East African and representative Asian and West African rice blast strains $\mathrm{s}^{\lambda}$

\begin{tabular}{lcccccccc}
\hline Population & $N$ & Gene diversity & Shannon's index & $\%$ Pol & $I_{A}$ & $r_{d}$ & MLG & Number of private alleles \\
\hline Tanzania & 14.0 & 0.12 & 0.19 & 49.0 & 4.19 & 0.05 & 14.0 & 2.0 \\
Uganda & 31.0 & 0.12 & 0.21 & 68.7 & 3.76 & 0.03 & 31.0 & 8.0 \\
Rwanda & 20.0 & 0.13 & 0.21 & 60.1 & 7.21 & 0.06 & 20.0 & 8.0 \\
West Africa & 8.0 & 0.09 & 0.15 & 31.3 & 9.94 & 0.17 & 5.0 & 0.0 \\
Asia & 15.0 & 0.13 & 0.20 & 48.5 & 3.93 & 0.04 & 15.0 & $\ldots .0$ \\
Mean & $\ldots$ & 0.13 & 0.24 & 51.52 & 5.81 & 0.07 & $\ldots$ & $\ldots$ \\
\hline
\end{tabular}

A Abbreviations: $N=$ the number of isolates, $\% \mathrm{Pol}=$ percentage polymorphism, $I_{A}=$ index of association, $r_{d}=$ standardized index of association, and $\mathrm{MLG}=$ multilocus genotypes.
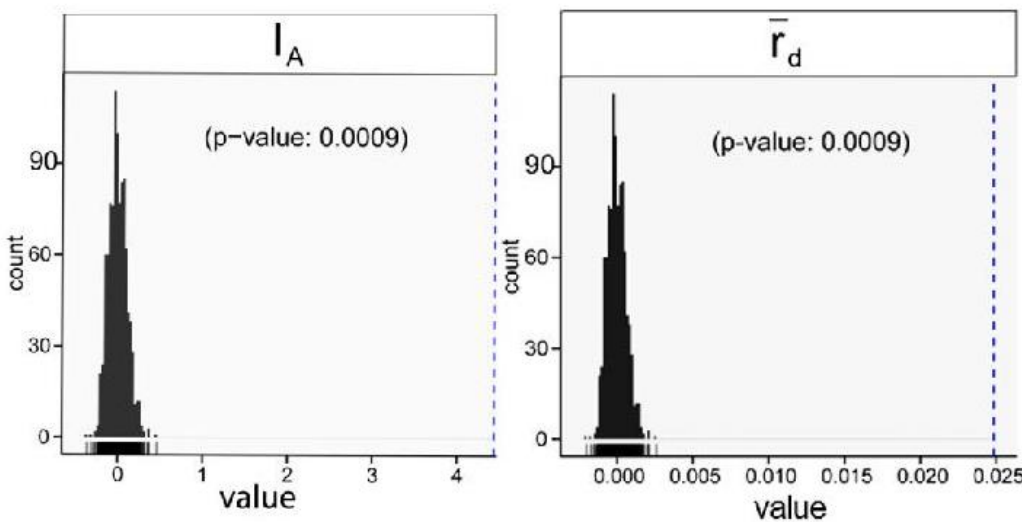

Fig. 2. Visualization of tests for linkage disequilibrium, where observed values (dashed lines) of $I_{A}$ and $r_{d}$ are compared with histograms showing results of 999 permutations using the poppr $\mathrm{R}$ package. All populations were clonal (observed value is significantly different from the expected panmixia).

TABLE 3. Genetic differentiation $\left(G_{S T}\right)$, gene flow $(\mathrm{Nm})$, genetic distance (GD), and genetic identity (I) among and between East African Magnaporthe oryzae populations and selected Asian and West African groups ${ }^{7}$

\begin{tabular}{|c|c|c|c|c|c|c|c|c|c|c|}
\hline \multirow[b]{2}{*}{ Population } & \multicolumn{5}{|c|}{$G_{S T}$} & \multicolumn{5}{|c|}{ I } \\
\hline & A & $\mathrm{R}$ & $\mathrm{U}$ & $\mathrm{T}$ & W & A & $\mathrm{R}$ & $\mathrm{U}$ & $\mathrm{T}$ & W \\
\hline Asia $(n=15)$ & $\ldots$ & 0.12 & 0.09 & 0.10 & 0.14 & $\ldots$ & 0.962 & 0.971 & 0.969 & 0.960 \\
\hline Rwanda $(n=20)$ & 3.72 & $\ldots$ & 0.03 & 0.04 & 0.08 & 0.039 & $\ldots$ & 0.992 & 0.987 & 0.979 \\
\hline Uganda $(n=31)$ & 4.79 & 16.78 & $\ldots$ & 0.04 & 0.09 & 0.030 & 0.009 & $\ldots$ & 0.990 & 0.977 \\
\hline Tanzania $(n=14)$ & 4.31 & 10.84 & 13.10 & $\ldots$ & 0.07 & 0.032 & 0.013 & 0.010 & $\ldots$ & 0.982 \\
\hline West Africa $(n=8)$ & 3.04 & 5.97 & $\begin{array}{l}5.17 \\
\mathrm{Nm}\end{array}$ & 7.06 & $\ldots$ & 0.041 & 0.021 & $\begin{array}{c}0.024 \\
\text { GD }\end{array}$ & 0.017 & $\ldots$ \\
\hline
\end{tabular}

Abbreviations: $\mathrm{A}=$ Asia, $\mathrm{R}=\mathrm{Rwanda}, \mathrm{U}=\mathrm{Uganda}, \mathrm{T}=$ Tanzania, $\mathrm{B}=$ Benin, and $\mathrm{N}=$ Nigeria. $G_{S T}$ and $\mathrm{I}$ are in the upper diagonals in the left and right panels of the table, respectively. Gene flow estimated as $G_{S T}=0.5\left(1-G_{S T}\right) / G_{S T}(N m) ; G D$ is in the lower diagonal of left and right panels, respectively.

TABLE 4. Results of analysis of molecular variance for populations of Magnaporthe oryzae sampled from East Africa, West Africa, and Asia

\begin{tabular}{|c|c|c|c|c|}
\hline Source of variation & df & Sum of squares & Variance components & Variation (\%) \\
\hline Among groups & 2 & 85.04 & $1.57 \mathrm{Va}$ & 10.97 \\
\hline Among populations within groups & 4 & 57.41 & $0.13 \mathrm{Vb}$ & 0.93 \\
\hline Within populations & 81 & 1021.25 & $12.61 \mathrm{Vc}$ & 88.09 \\
\hline Total & 87 & 1163.71 & 14.31 & $\ldots$ \\
\hline
\end{tabular}

1142 PHYTOPATHOLOGY 
is consistent with the $G_{S T}$ values. In addition, pairwise comparisons using genetic identity and genetic distances showed low genetic diversity, further indicating that $M$. oryzae populations at the studied sites were relatively homogenous and stable over the study period. Similar population structures have been observed in Europe

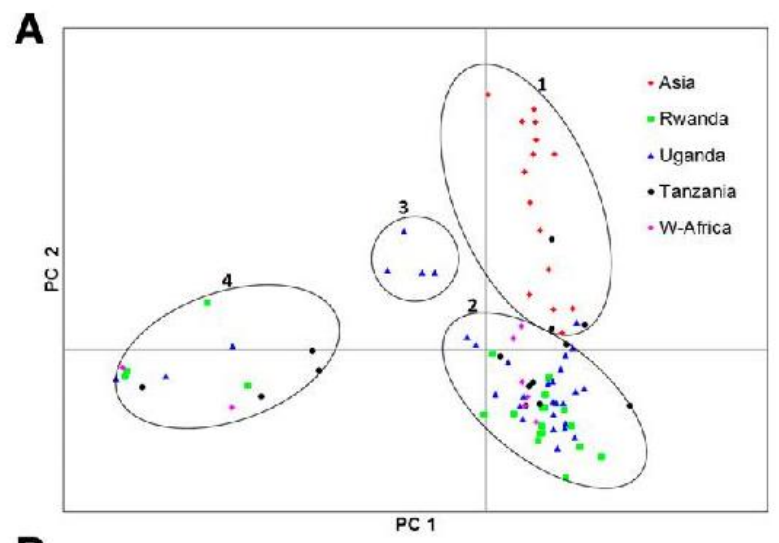

B

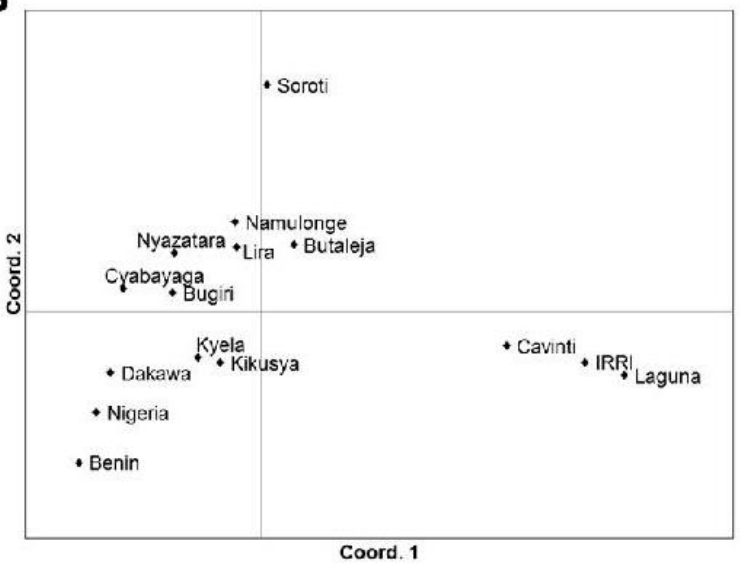

Fig. 3. A, Principal coordinate analysis of Magnaporthe oryzae isolates from East Africa and representative strains from Philippines and West Africa. The first and second principal coordinates account for 35.73 and $18.37 \%$ of the variation, respectively. Numbers 1, 2, 3, and 4 indicate cluster groupings. B, Principal coordinate analysis of $M$. oryzae subgroups from East Africa and representative strains from Philippines and West Africa. Isolates from the various collection sites are pooled into 15 subgroups. Subgroups Namulonge, Doho (Butaleja), Lira, and Tilda (Bugiri) represent Uganda; Nyazatara and Cyabayaga represent Rwanda; Dakawa, Kyela, and Kikusya represent Tanzania; Cavinti, IRRI, and Laguna represent Philippines; and Nigeria and Benin represent West Africa.
(Piotti et al. 2005; Roumen et al. 1997), Iran (Javan-Nikkah et al. 2004), the United States (Correll et al. 2009; Levy et al. 1991; Xia et al. 1993), Argentina (Consolo et al. 2008), Colombia (Levy et al. 1993; Zeigler 1998), Cuba (Fuentes et al. 2003), and West Africa (Takan et al. 2012), in which only a few lineages were detected. Therefore, it appears that either selection pressures have played a weak role in altering allele frequencies or a scenario similar to the aforementioned areas could be shaping the EA populations. Another explanation could be that any selection pressures that exist to influence allele frequencies are not confined to a particular geographic area in EA. On the other hand, $M$. oryzae has been present in EA for over 90 years, and this provides less direct but intriguing evidence that the effect of a colonization bottleneck and subsequent clonal growth could have shaped the EA populations.

The use of hierarchical AMOVA (Table 4) further supports these findings, and corresponds with the strong indication of gene flow detected in this study. Gene flow involves the introduction or reintroduction of genes to a population, and our results indicate that gene flow in EA populations is comparable with that reported for geographically distant wheat blast populations in Brazil (Maciel et al. 2014) and other ascomycete fungi (e.g., Mycosphaerella graminicola) (Zhan et al. 2003). This evidence for extensive gene flow in Magnaporthe oryzae indicates the ongoing connection between the EA populations, though alternative explanations are also possible. There may be two possible scenarios: either the high gene flow results from the movement of planting materials between these countries or from movement of $M$. oryzae strains to EA via different other routes or at different times but from a common source, homogenizing the effect of gene flow. If germplasm movement is the cause of high gene flow, we speculate that Uganda could represent a focal point from which the pathogen was established first, considering the relatively high gene diversity and the striking admixture (Fig. 4B). However, due to the low sample size of isolates from the other countries studied, such analyses will have to be carefully considered, and continued genetic analysis of additional samples, as they become available, will help to verify this argument. Thus, the genetic clustering observed in our data may reflect either germplasm movement or historical patterns of gene flow that occurred decades ago, which could be both long-lived and able to propagate through asexual reproduction. In both cases, regional efforts to abate disease outbreaks through joint resistance breeding are plausible.

The PCoA, based on Nei's genetic distance, captured only $54.1 \%$ of the total variation in two principal components, suggesting limited robustness in the strain grouping. Only the spatial analysis of principal components revealed a slight geographical pattern among the subpopulations. The clustering pattern generated from STRUCTURE 2.3 also did not follow the geographical origin, except for isolates from Philippines, thus supporting the mixed pattern depicted in PCoA. The results obtained from STRUCTURE also show that isolates from Uganda are distributed in all clusters,
A

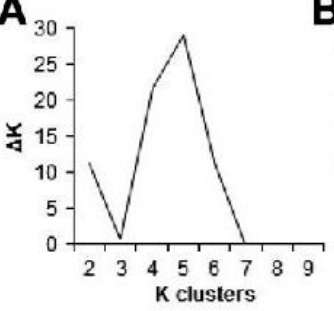

B

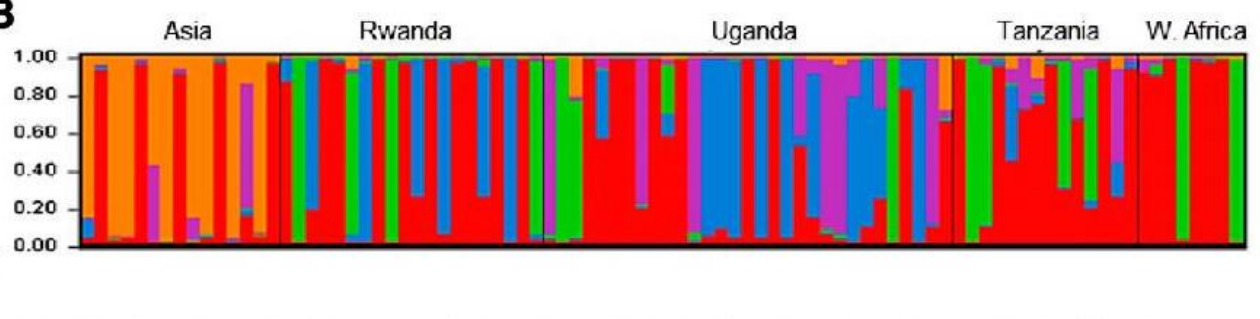

Fig. 4. Graphical representation of the results obtained from STRUCTURE. A, The most probable value of $\mathrm{K}$, determined by $\Delta \mathrm{K}$ for 88 strains $(x$-axis $)$ sorted by membership coefficients ( $y$-axis) within clusters $(K=5)$. B, STRUCTURE plot of the 88 isolates with $\mathrm{K}=5$ clusters. The plot is sorted according to the origin of isolates. Different colors represent genetic groups. Each column represents an individual partitioned into segments representing the estimated coefficients of membership proportions in the five ancestral genetic clusters (genetic groups) inferred with STRUCTURE. The height of the column segments shows the probability of assignment of individual isolates to the genetic groups based on 100,000 runs. 
and is consistent with the historical reports of the first detection of the pathogen in Africa (Small 1922), although it would have been present in earlier times than 1921.

Interestingly, both MAT1-1 and MAT1-2 were detected, although with location-specific differences and skewed ratio toward MAT1-1 in all EA populations. Such a skewed pattern of MAT distribution has been also observed in West Africa (Takan et al. 2012). It is common for one MAT to be dominant in field populations, with most of the isolates being male fertile or completely sterile (Tredway et al. 2005). Moreover, recombinants are rarely reported in such scenarios. Whether the EA strains are able to reproduce sexually remains to be determined. However, the skewed distribution of the two MAT and the significant and positive $I_{A}$ values suggest clonal or mixed reproduction within populations but not sexual reproduction. Thus, we hypothesize that the introduction of isolates with two MAT could have occurred in EA. However, the exact localization of the source population and the time of introduction of both MAT remain to be determined. The difference in MAT distribution between Philippine and most EA isolates suggests that EA isolates could have originated from a source other than the Philippines, which may require a substantially large sample size to verify, including isolates from regions other than the Philippines. Several lines of research that pertain to this issue are in progress that will further our understanding of $M$. oryzae origin and distribution in EA.

In our pathogenicity study, isolates showed differences in severity and disease progress. By comparison of virulence on Co39, Tanzania and $\mathrm{R}$ wanda had a higher percentage of virulent isolates than Uganda. However, the high pathogenic variability was not consistent with the population structure, suggesting that $M$. oryzae populations in EA are dominated by genetically less diverse strains but diverse pathotypes, which is similar to the populations previously reported in Japan (Don et al. 1999) and South Korea (Parket al. 2003). This scenario suggests that pathogenicity is labile to be predicted by linkage with neutral markers in these population samples.

Overall, our results showed low genetic differentiation, skewed distribution of the two MAT, and a possible role of gene flow in $M$. oryzae populations from EA. Either $M$. oryzae could be distributed as a single genetic population in EA or that gene flow is exerting a significant influence, effectively swamping the action of selection. In this way, the alleles present in Tanzania, Uganda, and Rwanda are primarily subsets, with the exception of a few new alleles which could have been created by mutations. These rare alleles could be the reason for the variable pathogenicity observed in EA populations. Although AFLP screens a large number of polymorphic loci, diversity estimates reported in the present study will have to be carefully considered and may need to be confirmed with other marker systems, which could broaden the molecular understanding of the EA pathogen population. Future research should also focus on identifying the source population and study further the evolutionary potential of EA isolates with additional sampling and long-term monitoring. The use of a standard set of differential rice lines with single resistance genes for race differentiation would provide further evidence for pathogenic diversity. The effect of high temperature associated with climate change on recent rice blast outbreaks observed in EA will have to be carefully considered because little is known about the biology of this pathogen in EA.

\section{ACKNOWLEDGMENTS}

This work was funded by the Germany Federal Ministry of Economic Cooperation and Development, Deutsche Gesellschaft für Internationale Zusammenarbeit GmbH. We thank P. Karlovsky for his helpful suggestions for this work; R. Pilot, E. Vorbeck, and D. Tacke for their technical support; C. Kukunda for help in drawing the map; and N. Grünwald for help in data analysis.

\section{LITERATURE CITED}

Borromeo, E. S., Nelson, R. J., Bonman, J. M., and Leung, H. 1993. Genetic differentiation among isolates of Pyricularia infecting rice and weed hosts. Phytopathology 83:393-399.

Brandfass, C., and Karlovsky, P. 2008. Upscaled CTAB-based DNA extraction and real-time PCR assays for Fusarium culmorum and F. graminearum DNA in plant material with reduced sampling error. Int. J. Mol. Sci. 9: 2306-2321.

Brondani, C., Brondani, R. P. V., Garrido, L., Da, R., and Ferreira, M. E. 2000. Development of microsatellite markers for genetic analysis of Magnaporthe grisea. Genet. Mol. Biol. 23:753-762.

Campbell, C. L., and Madden, L. V. 1990. Introduction to Plant Disease Epidemiology. John Wiley \& Sons, New York.

Chen, D., Zeigler, R. S., Leung, H., and Nelson, R. J. 1995. Population structure of Pyricularia grisea at two screening sites in the Philippines. Phytopathology 85:1011-1020.

Consolo, V. F., Cordo, C. A., and Salerno, G. L. 2008. DNA fingerprint and pathotype diversity of Pyricularia oryzae populations from Argentina. Austral. Plant Pathol. 37:357-364.

Consolo, V. F., Cordo, C. A., and Salerno, G. L. 2005. Mating-type distribution and fertility status in Magnaporthe grisea populations from Argentina. Mycopathologia 160:285-290.

Correll, J. C., Boza, E. J., Seyran, E., Cartwright, R. D., Jia, Y., and Lee, F. N. 2009. Examination of the rice blast pathogen population diversity in Arkansas, USA-Stable or unstable? Pages 217-228 in: Advances in Genetics, Genomics and Control of Rice Blast Disease. G.-L. Wang and B. Valent, eds. Springer, New York.

Couch, B. C., Fudal, I., Lebrun, M. H., Tharreau, D., Valent, B., van Kim, P., Nottéghem, J. L., and Kohn, L. 2005. Origins of host-specific populations of the blast pathogen Magnaporthe oryzae in crop domestication with subsequent expansion of pandemic clones on rice and weeds of rice. Genetics 170:613-630.

Don, L. D., Urashima, A. S., Tosa, Y., Nakayashiki, H., and Mayama, S. 1999. Population structure of the rice blast fungus in Japan examined by DNA fingerprinting. Ann. Phytopathological Soc. Jpn. 65 : 15-24.

Evanno, G., Regnaut, S., and Goudet, J. 2005. Detecting the number of clusters of individuals using the software structure: A simulation study. Mol. Ecol. 14:2611-2620.

Excoffier, L., Laval, G., and Schneider, S. 2005. Arlequin 3.01: An integrated software package for population genetics data analysis. Evol. Bioinf. Online 1:47-50.

FAOSTAT. 2011 . Rough rice area ( $000 \mathrm{ha})$, by country and geographical region, 1961-2011. Food and Agricultural Organization of the United Nations-Production Statistics. http://faostat.fao.org/site/567/desktopdefault. aspx\#ancor

Fuentes, J. L., Correa-Victoria, F. J., Escobar, F., Mora, L., Duque, M. C., Deus, J. E., and Cornide, M. T. 2003. Genetic diversity analysis of the rice blast pathogen population at two locations in Cuba. Biotecnol. Apl. 20: 14-19.

Gerber, S., Mariette, S., Streiff, R., Bodénès, C., and Kremer, A. 2000. Comparison of microsatellites and amplified fragment length polymorphism markers for parentage analysis. Mol. Ecol. 9:1037-1048.

Grünwald, N. J., Goodwin, S. B., Milgroom, M. G., and Fry, W. E. 2003. Analysis of genotypic diversity data for populations of microorganisms. Phytopathology 93:738-746.

Javan-Nikkah, M., McDonald, B. A., Banke, S., and Hedjaroude, G.-A. 2004. Genetic structure of Iranian Pyricularia grisea populations based on repPCR fingerprinting. Eur. J. Plant Pathol. 110:909-919.

Jost, L. 2008. GST and its relatives do not measure differentiation. Mol. Ecol. 17:4015-4026.

Kachroo, P., Leong, S. A., and Chattoo, B. B. 1994. Pot2, an inverted repeat transposon from the rice blast fungus Magnaporthe grisea. Mol. Gen. Genet. 245:339-348

Kihoro, J., Njoroge, J. B., Murage, H., Ateka, E., and Makihara, D. 2013. Investigating the impact of rice blast disease on the livelihood of the local farmers in greater Mwea region of Kenya. SpringerPlus 2:308.

Kumar, J., Nelson, R. J., and Zeigler, R. S. 1999. Population structure and dynamics of Magnaporthe grisea in the Indian Himalayas. Genetics 152:971-984.

Levy, M., Correa-Victoria, F. J., Zeigler, R. S., Hu, S., and Hamer, J. E. 1993. Genetic diversity of the rice blast fungus in a disease nursery in Colombia. Phytopathology 83:1427-1433.

Levy, M., Romao, J., Marchetti, M. A., and Hamer, J. E. 1991. DNA fingerprinting with dispersed repeated sequence resolves pathotype diversity in the rice blast fungus. Plant Cell 3:95-102.

Liu, J., Wang, X., Mitchell, T., Hu, Y., Liu, X., Dai, L., and Wang, G. L. 2010. Recent progress and understanding of the molecular mechanisms of the rice-Magnaporthe oryzae interaction. Mol. Plant Pathol. 11:419-427. 
Liu, Z. J., and Cordes, J. F. 2004. DNA marker technologies and their applications in aquaculture genetics. Aquaculture 238:1-37.

Maciel, J. L., Ceresini, P. C., Castroagudin, V. L., Zala, M., Kema, G. H., and McDonald, B. A. 2014. Population structure and pathotype diversity of the wheat blast pathogen Magnaporthe oryzae 25 years after its emergence in Brazil. Phytopathology 104:95-107.

Miller, M. P. 1997. Tools for Population Genetic Analyses (TFPGA) v. 1.3: A Windows Program for the Analysis of Allozyme and Molecular Population Genetic Data. Department of Biological Sciences, Northern Arizona University. http://www.ccg.unam.mx/ vinuesa/tlem09/docs/TFPGADOC.PDF

Mwalyego, F. S., Kayeke, J. M., and Mghogho, R. M. 2011. ASARECA project: Livelihood improvement through integrated management practices for rainfed lowland rice. A guide for farmers. http://www.erails. net/images/tanzania/ari-uyle/ari-uyole/file/Rice\%20diseases\%202011.pdf

Nei, M. 1978. Estimation of average heterozygosity and genetic distance from a small number of individuals. Genetics 89:583-590.

Okori, P., Rubaihayo, P. R., Adipala, E., Fahleson, J., and Dixelius, C. 2004. Population studies of fungal pathogens: Perspectives for control with specific reference to grey leaf spot. Afr. Crop Sci. J. 12:327-342.

Park, S.-Y., Milgroom, M. G., Han, S. S., Kang, S., and Lee, Y.-H. 2003. Diversity of pathotypes and DNA fingerprint haplotypes in populations of Magnaporthe grisea in Korea over two decades. Phytopathology 93:1378-1385.

Peakall, R., and Smouse, P. E. 2006. GENEALEX 6: Genetic analysis in excel. Population genetic software for teaching and research. Mol. Ecol. Notes 6: 288-295.

Piotti, E., Rigano, M. M., Rodino, D., Rodolfi, M., Castiglione, S., Picco, A. M., and Sala, F. 2005. Genetic structure of Pyricularia grisea (Cooke) Sacc. isolates from Italian paddy fields. J. Phytopathol. 153:80-86.

Pritchard, J. K., Stephens, M., and Donnelly, P. 2000. Inference of population structure using multilocus genotype data. Genetics 155:945-959.

Roumen, E., Levy, M., and Notteghem, J. L. 1997. Characterization of the European pathogen population of Magnaporthe grisea by DNA fingerprinting and pathotype analysis. Eur. J. Plant Pathol. 103:363-371.

Saleh, D., Milazzo, J., Adreit, H., Fournier, E., and Tharreau, D. 2014. SouthEast Asia is the center of origin, diversity and dispersion of the rice blast fungus, Magnaporthe oryzae. New Phytol. 201:1440-1456.

Sere, Y., Onasanya, A., Afolabi, A., Mignouna, H. D., and Akator, K. 2007. Genetic diversity of the blast fungus, Magnaporthe grisea (Hebert) Barr. in Burkina Faso. Afr. J. Biotechnol. 6:2568-2577.

Silva G. B., Prabhu, A. S., Filippi, M. C. C., Trindade M. G., Araújo, L. G., and Zambolim, L. 2009. Genetic and phenotypic diversity of Magnaporthe oryzae from leaves and panicles of rice in commercial fields in the State of Goiás, Brazil. Trop. Plant Pathol. 34:71-76.

Small, W. 1922. Annual report of the government mycologist for 1921. An investigation into fungi found on rice in Uganda. Uganda Prot. Dep. Agric, Annu. Rep. Pages 49-57. Rev. Appl. Mycol. 2:156-157.

Stoddart, J. A., and Taylor, J. F. 1988. Genotypic diversity: Estimation and prediction in samples. Genetics 118:705-711.

Takan, J. P., Chipili, J., Muthumeenakshi, S., Talbot, N. J., Manyasa, E. O., Bandyopadhyay, R., Sere, Y., Nutsugah, S. K., Talhinhas, P., Hossain, M., Brown, A. E., and Sreenivasaprasad, S. 2012. Magnaporthe oryzae populations adapted to finger millet and rice exhibit distinctive patterns of genetic diversity, sexuality and host interaction. Mol. Biotechnol. 50: 145-158.

Thuan, T. N. T., Bigirimana, J., Roumen, E., Van Der Straeten, D., and Höfte, M. 2006. Molecular and pathotype analysis of the rice blast fungus in North Vietnam. Eur. J. Plant Pathol. 114:381-396.

Tredway, L. P., Stevenson, K. L., and Burpee, L. L. 2005. Genetic structure of Magnaporthe grisea population associated with St. Augustine grass and tall fescue in Georgia. Phytopathology 95:463-471.

Vos, P., Hogers, R., Bleeker, M., Reijans, M., Vanderlee, T., Hornes, M., Frijters, A., Pot, J., Peleman, J., Kuiper, M., and Zabeau, M. 1995. AFLP: New technique for DNA-fingerprinting. Nucleic Acids Res. 23:4407-4414,

Weiberg, A., and Karlovsky, P. 2009. Components of variance in transcriptomics based on electrophoretic separation of cDNA fragments (cDNA-AFLP). Electrophoresis 30:2549-2557.

Xia, J. Q., Correll, J., Lee, F. N., and Ross, W. J. 2000. Regional population diversity of Pyricularia grisea in Arkansas and the influence of host selection. Plant Dis. 84:877-884

Xia, J. Q., Correll, J. C., Lee, F. N., Marchetti, M. A., and Rhoads, D. D. 1993. DNA fingerprinting to examine microgeographic variation in the Magnaporthe grisea population in two rice fields in Arkansas. Phytopathology 83: 1029-1035.

Yeh, F. C., and Boyle, T. 1999. POPGENE version 1.3.2: Microsoft windowbased freeware for population genetic analysis. Online publication. https://www. ualberta.ca/ fyeh/popgene.html

Zeigler, R. 1998. Recombination in Magnaporthe grisea. Annu. Rev. Phytopathol. 36:249-275.

Zhan, J., Pettway, R. E., and McDonald, B. A. 2003. The global genetic structure of the wheat pathogen Mycosphaerella graminicola is characterized by high nuclear diversity, low mitochondrial diversity, regular recombination and gene flow. Fungal Genet. Biol. 38:286-297. 
Chapter 5: General discussion

Little is known about the mechanisms underlying rice response to $M$. oryzae under high temperature. Phenotypic and transcriptome analysis enabled us to determine whether prolonged exposure to $35^{\circ} \mathrm{C}$ prior to infection has consequences on the ability of rice to resist infection. Because high temperature is known to affect the physiological functioning of plants (Hasanuzzaman et al., 2013), probably including defense responses to other stresses, it was expected that plants would universally succumb to pathogen infection after high temperature exposure due to prior metabolic expenditure. However, our data revealed that high temperature, despite reduced expression of the $\mathrm{R}$ gene (Pi54) at $35^{\circ} \mathrm{C}$ compared to $28^{\circ} \mathrm{C}$, induced resistance in the rice genotypes LT and CO. This observation indicated that other genetic factors could be interacting with Pi54 to mediate resistance. These may include resistance gene analogues (RGAs) which co-localize with Pi54 on chromosome 11, including RPM1. Hence, the weak expression of Pi54 at high temperature may nonetheless contribute to resistance when combined with high temperature activated RGAs. The likelihood of $\mathrm{R}$ gene interaction to activate several resistance pathways is, therefore, not farfetched, though at present, there is no clear experimental evidence demonstrating how two or more NB-LRR proteins augment each other, except for RPS2 and RPM1 mediated hypersensitive response (Jorgensen and Emerson, 2008). Besides this, we detected high accumulation of $\mathrm{H}_{2} \mathrm{O}_{2}$ and increased expression of $\mathrm{ABA}$ responsive genes in plants exposed to $35^{\circ} \mathrm{C}$. Previous reports have shown that $\mathrm{H}_{2} \mathrm{O}_{2}$ accumulates in forming papillae and can be used by peroxidases to promote cross-linking of proteins and phenolics to reinforce cell wall appositions (ThordalChristensen et al., 1997; Brown et al., 1998; Voigt, 2014). The early induction of $\mathrm{H}_{2} \mathrm{O}_{2}$ and anti-oxidative mechanisms that protect the plant against high temperature, including POX, most likely remains active to limit both pathogen invasion and further oxidative stress, while diverting the prevailing $\mathrm{H}_{2} \mathrm{O}_{2}$ for cell wall appositions. The induction of callose by $M$. oryzae infection is apparently co-regulated by high temperature ROS induced ABA production, which is consistent with the induction of OsBBD1 in our study.

In our gene expression analysis, we also detected high expression levels of several genes representing diverse biological pathways. The most compelling was the high expression levels of OsBISAMT1, which converts SA to MeSA, across all genetic backgrounds during infection. Moreover, enhanced expression was detected in plants exposed to $35^{\circ} \mathrm{C}$ compared to $28^{\circ} \mathrm{C}$. It is unknown whether conversion of SA to MeSA is a strategy to reduce or enhance $\mathrm{H}_{2} \mathrm{O}_{2}$ production, given that positive regulation of $\mathrm{H}_{2} \mathrm{O}_{2}$ by SA has been reported (Rao et al., 
1997). On the other hand, increased production of $\mathrm{H}_{2} \mathrm{O}_{2}$ has been shown to stimulate $\mathrm{SA}$ production (Chamnongpol et al., 1998). The regulatory networking between the two molecules is apparently rate limiting. However, if the conversion of SA to MeSA affects $\mathrm{H}_{2} \mathrm{O}_{2}$ accumulation, probably negating plant resistance, while protecting the plant from heat stress, then other mechanisms must be in place to mitigate the subsequent pathogen infection. Previous reports have shown that SAR establishment may require signals, which precede systemic accumulation of SA, such as jasmonates (Truman et al., 2007) and indole-derived metabolites (Truman et al., 2010). Furthermore, Liu et al., (2011) suggested that MeSA and a complex formed between the lipid transfer protein DIR1, glycerol lipid and lipid derivatives mediate SAR. We speculate that MeJA could be linked to SAR, reminiscent of a strong induction of jasmonate-O-methyltransferase in LT. Downstream of ROS, MeSA and MeJA there apparently exists a complex signaling network with probable complementary and inhibitory roles. How the plant processes these signals is apparently reliant on the effect of the genetic background during pathogen infection. For instance, despite carrying the same $\mathrm{R}$ gene (Pi54), LT and CO showed contrasting reaction to $M$. oryzae at $28^{\circ} \mathrm{C}$, with $\mathrm{CO}$ displaying a more severe disease phenotype compared to LT. In addition, LT showed more intense cell wall florescence than $\mathrm{CO}$. These observations were also reflected at the gene expression level, where some resistance genes, including a jacalin-like lectin (LOC_Os12g14440) that mediates broad spectrum resistance, naringenin 7-O-methyltransferase for sakuranetin biosynthesis and RGAs were background specific. This suggests that the genetic background plays a role in plant defense induction, and that some cultivars may have a more effective resistance against M. oryzae than others despite varying temperatures.

This was further intriguing in the second experiment where we examined the possible direct effects of temperature on $M$. oryzae infection in Nipponbare. As reflected by the severe disease phenotype, infection success was higher at $35^{\circ} \mathrm{C}$ compared to $28^{\circ} \mathrm{C}$. This indicates that the resistance of Nipponbare against $M$. oryzae is negatively affected by high temperature. High temperatures can alter cell properties, including the phospholipid membrane surrounding the cytosol and internal organelles. The effect may translate into increased permeability affecting the ability of other molecules such as signaling proteins to interact with phospholipid membranes. Such changes, if they occur in the host, alter what we termed as conserved pathogen recognition patterns (CPRPs). These membrane changes and the subsequent defense responses are apparently subject to genotype specific traits which may subsequently determine infection success of the pathogen. 
Predicting the impact of temperature is, however, challenging as it may also affect $M$. oryzae pathogenicity. For instance, the observed effect of high temperature on rapid disease progression and elevated expression of putative pathogenicity genes may be related to altered pathogen virulence, hyphal structural adjustments and survival strategies in a stress host environment. Our data revealed more than 100 transcripts encoding for hydrolytic enzymes including glycosyl hydrolases (pectinase, amylases, cellulases, xylanases, chitinases, glucanases), peptidases, lipases and phospholipases, all of which have been found to be involved in pathogenicity of many fungal pathogens (King et al., 2011). Thus, an increase in pathogenicity related factors likely enables $M$. oryzae to quickly adapt and overcome host defense, depending on the host genetic factors. Several genes, including Avr genes appear to be indispensable for $M$. oryzae infection regardless of the temperature difference, given their high expression levels in both Nip35i and Nip28i. However, the relatively higher expression in Nip35i compared to Nip28i suggests that the expression of a cognate $\mathrm{R}$ gene, even at reduced levels, is likely to detect the pathogen effectors and subsequently evoke ETI. This is consistent with the lower expression of Pi54 in CO and LT at $35^{\circ} \mathrm{C}$, and alternatively, a higher induction of $A v r P i k / k m / k p$ in Nip35i. This indicates that the expression level of an $a v r$ gene can determine the plant capacity to detect the pathogen. Thus, $\mathrm{R}$ gene-mediated resistance at high temperature may depend on the $a v r$ gene dosage effect.

As we have not yet demonstrated the function of the genes revealed in this study, we can only speculate that the plant and pathogen specific genes induced in the rice-M. oryzae interaction are apparently highly interactive in conferring resistance and virulence at high temperature. Overall, our results suggest that the effects of high temperature on host resistance and pathogen infection success may be overridden by the genetic background of the host. Functional analysis of background modifier genes, including constitutively expressed NBSLRRs, and OMTs could provide further insight into the mechanism of resistance relevant for improving rice health in a changing climate.

We also analyzed the population structure of M. oryzae in East Africa using amplified fragment length polymorphism (AFLP) markers, to provide a basis for future studies into the relationship between climate change and the forces that shape the population structure. Our results indicate low genetic differentiation in East African populations. Besides, pair-wise comparisons using $\mathrm{G}_{\mathrm{ST}}$, genetic identity and genetic distances also showed low genetic diversity, suggesting that $M$. oryzae populations at the studied sites were relatively homogenous and stable over the study period. It appears therefore that selection pressures 
have played a weak role in altering allele frequencies on the East African populations. It is also tempting to suggest that any selection pressures that exist to influence allele frequencies are not confined to a particular geographic area. On the other hand, a strong indication of gene flow was detected among East African populations. Gene flow involves the introduction or reintroduction of genes to a population. By moving genes around, there is a potential for the spread of virulent pathotypes with highly advantageous alleles, which might contribute to drastic crop losses. Regional efforts to limit informal movement of planting materials may be necessary to prevent epidemics associated with exotic strains. The presence of two mating types in some sites further suggests that there is a possibility of the fungus to adapt to heterogenous hosts or environments quickly, and could lead to the development of novel virulences. This could be the reason for variable pathogenicities within populations analyzed in this study.

Overall, our transcriptome analysis provides a broad overview of genes and biological processes involved in rice response to high temperature and $M$. oryzae, and contain representatives of all of the major classes of defense genes. We show that different rice genetic backgrounds modify the defense response in rice. The defense response is compounded by the interaction between temperature and the genetic background, and apparently regulates the nature, timing and magnitude of ROS, hormone signaling pathways and the subsequent defense responses to M. oryzae. Thus, high temperature effect on cultivar response to $M$. oryzae may not be consistently similar across genotypes. Furthermore, the activation of defense response genes and infection success of the pathogen will be more dependent on the genotypes used as cultivars, and the degree of their adaptation to both high temperature and pathogen infection without tradeoffs.

On the other hand, M. oryzae populations from East Africa are apparently exposed to lowly variable climatic conditions, hosts and cropping systems, as indicated by relatively homogenous populations. This suggests that high temperature associated with climate change may not typically be a cause of the recent sporadic rice blast out breaks observed in East Africa, but could be related to resistance breakdown associated with old traditional cultivars; and probably genetic changes in the pathogen that could not be detected by AFLP analysis. 


\section{References}

Brown, I., Trethowan, J., Kerry, M., Mansfield, J., and Bolwell, G. P. (1998) Localization of components of the oxidative cross-linking of glycoproteins and of callose synthesis in papillae formed during the interaction between non-pathogenic strains of Xanthomonas campestris and French bean mesophyll cells. Plant J. 15, 333-343.

Dat, J.F., Lopez-Delgado, H., Foyer, C.H., Scott, I.M. (1998) Parallel change in $\mathrm{H}_{2} \mathrm{O}_{2}$ and catalase during thermotolerance induced by salicylic acid or heat acclimation in mustard seedlings. Plant Physiol. 116, 1351-1357.

Hasanuzzaman, M., Nahar, K., Alam, M.d.M., Roychowdhury, Rajib., and Fujita, M. (2013) Physiological, biochemical, and molecular mechanisms of heat stress tolerance in plants. Int J Mol Sci. 14, 9643-9684.

Jorgensen, T.H., Emerson, B.C. (2008) Functional variation in a disease resistance gene in populations of Arabidopsis thaliana. Molecular Ecology 17, 4912-4923.

King, B.C., Waxman, K.D., Nenni, N.V., Walker, L.P., Bergstrom, G.C., Gibson, D.M. (2011) Arsenal of plant cell wall degrading enzymes reflects host preference among plant pathogenic fungi. Biotechnol. Biofuels 4, 4.

Liu, P.P., von Dahl, C.C., Park, S.W., Klessig, D.F. (2011) Interconnection between methyl salicylate and lipid-based long-distance signaling during the development of systemic acquired resistance in Arabidopsis and tobacco. Plant Physiol. 155, 1762-1768.

Rao, M.V., Paliyath, G., Ormrod, D., Murr, D.P., Watkins, C.B. (1997) Influence of salicylic acid on $\mathrm{H} 2 \mathrm{O} 2$ production, oxidative stress and $\mathrm{H} 2 \mathrm{O} 2$ metabolizing enzymes: salicylic acid-mediated oxidative damage requires H2O2. Plant Physiol 115, 137-149.

Thordal-Christensen, H., Zhang, Z., Wei, Y., and Collinge, D. B. (1997) Subcellular localization of $\mathrm{H} 2 \mathrm{O} 2$ in plants. $\mathrm{H} 2 \mathrm{O} 2$ accumulation in papillae and hypersensitive response during the barley-powdery mildew interaction. Plant J. 11, 1187-1194.

Truman, W., Bennett, M.H., Kubigsteltig,. I, Turnbull, C., Grant, M. (2007) Arabidopsis systemic immunity uses conserved defence signaling pathways and is mediated by jasmonates. Proc Natl Acad Sci USA, 104, 1075-1080.

Truman, W.M., Bennett, M.H., Turnbull, C.G.N., Grant, M.R. (2010) Arabidopsis auxin mutants are compromised in systemic acquired resistance and exhibit aberrant accumulation of various indolic compounds. Plant Physiol 152, 1562-1573. 
Summary

It is currently hypothesized that climate change may lead to global warming, and temperatures might increase by $2-5^{\circ} \mathrm{C}$ at the end of the twenty-first century. However, the consequences of the expected temperature elevation on pathogen biology, epidemiology and plant host resistance have continued to provide conflicting results due to several interacting factors. The rice- $M$. oryzae pathosystem is well-studied at both the phenotypic and genomic level, and the scientific advancement in both organisms makes it suitable for understanding the mechanisms underlying plant pathogen interaction at high temperature. In the first part of this study, we analyzed the host phenotypic reactions and RNA-seq genome-wide transcript profiles of two genetic rice backgrounds, CO (O. sativa indica; Co39) and LT (O. sativa japonica; LTH), carrying the same R gene (Pi54), and exposed to M. oryzae infection, after exposure to $28^{\circ} \mathrm{C}$ and $35^{\circ} \mathrm{C}$. Both backgrounds were more resistant at $35^{\circ} \mathrm{C}$ compared to $28^{\circ} \mathrm{C}$. Moreover, callose deposits and cell wall fluorescence of the attacked epidermal cells were more intense at $35^{\circ} \mathrm{C}$ than at $28^{\circ} \mathrm{C}$. However, $\mathrm{CO}$ had a more severe disease phenotype compared to $\mathrm{LT}$ at $28^{\circ} \mathrm{C}$. The differential reaction of Pi54 in the two backgrounds suggests that genetic factors other than Pi54 determine the resistance response, and further indicates that the genetic background of japonica rice facilitates the function of Pi54 more than the background of indica rice. Gene expression analysis further revealed substantial background transcriptional profile variation, and demonstrated that LT and $\mathrm{CO}$ exhibit differences in genes expressed in response to high temperature and M. oryzae. Hydrogen peroxide $\left(\mathrm{H}_{2} \mathrm{O}_{2}\right)$ production and abscisic acid (ABA) responsive genes were more pronounced in LT compared to $\mathrm{CO}$, whereas SA levels were higher in CO than LT. Both genotypes showed a high induction of OsBISAMT1, which converts SA to MeSA. Conversely, only LT showed a significant induction of jasmonate O-methyltransferase and JA induced jacalin-like lectin (LOC_Os12g14440) that mediates broad spectrum resistance. Thus, the nature, timing and magnitude of ROS and hormone signaling pathways in response to both high temperature and M. oryzae infection may be largely controlled by the genetic backgrounds.

In the second study, we analyzed the effect of temperature on the transcriptomes of the rice cultivar Nipponbare and its pathogen $M$. oryzae simultaneously at $35^{\circ} \mathrm{C}$ and $28^{\circ} \mathrm{C}$ using RNAseq. Our results showed that exposure of $M$. oryzae to $35^{\circ} \mathrm{C}$ increased the number and expression levels of putative effectors compared to infection at $28^{\circ} \mathrm{C}$. Genes involved in secondary metabolism, transport, lipid metabolism and carbohydrate metabolism also showed 
increased transcription during infection at $35^{\circ} \mathrm{C}$ compared to $28^{\circ} \mathrm{C}$. These transcription changes likely facilitated host colonization and adaptation. Indeed, we observed a more severe disease phenotype in Niponbare during $M$. oryzae infection at $35^{\circ} \mathrm{C}$ compared to $28^{\circ} \mathrm{C}$. Moreover, in planta fungal biomass was significantly higher in Nip35i than Nip28i, suggesting rapid colonization of the host tissues at $35^{\circ} \mathrm{C}$. The effect of high temperature on plant cell wall loosening probably facilitated rapid colonization and delivery of several proteins able to promptly manipulate the plant defense, as well as to degrade the cell wall. Several genes, including $A v r$ genes appear to be indispensable for $M$. oryzae infection at high temperature, given their high expression levels at $35^{\circ} \mathrm{C}$ compared to $28^{\circ} \mathrm{C}$. This suggests that the expression of a cognate $\mathrm{R}$ gene, even at reduced levels, is likely to detect the pathogen effectors and subsequently evoke ETI. This is consistent with the lower expression of Pi54 in $\mathrm{CO}$ and $\mathrm{LT}$ at $35^{\circ} \mathrm{C}$, and alternatively, a higher induction of $A v r P i k / \mathrm{km} / \mathrm{kp}$ in Nip35i. The expression level of an $a v r$ gene therefore determines the plant capacity to detect the pathogen under abiotic stresses that tend to reduce transcript levels of the $\mathrm{R}$ gene. Thus, $\mathrm{R}$ genemediated resistance at high temperature may depend on the avr gene dosage effect. Because the $P i k / k m / k p$ allele, which corresponds with a $A v r P i k / k m / k p$ like gene in $M$. oryzae are redundant or missing in Nipponbare, it is likely that the high temperature effect on plant cell wall and membrane permeability provided a direct pass for the pathogen effectors.

Taken together, the inability of the plant to defend itself when exposed to both abiotic and biotic stresses is correlated with the absence or the degree at which abiotic stress alters both specific and general defense responses. On the other hand, temperature effect on both $\mathrm{R}$ gene mediated resistance and partial resistance is likely more variable than earlier envisaged, and will require intensive screening efforts to identify stable resistant gene combinations. Thus, it may be too soon to generalize the effect of the changing temperature on host resistance beyond the current study because of the significant differences in defense responses among host genotypes studied so far. The likely scenario is that some cultivars may have a more stable resistance against $M$. oryzae than others despite increasing temperatures, and research efforts to identify such stable genotypes will need to be raised.

In the third part of this study, we analyzed the genetic diversity and population structure of 88 isolates of M. oryzae from East Africa and representative isolates from West Africa and the Philippines using amplified fragment length polymorphism (AFLP) markers. Our results indicate low genetic differentiation in East African populations, suggesting that the 
populations are relatively homogeneous. It appears that selection pressures have played a weak role in altering allele frequencies on the East African populations. It is also tempting to suggest that any selection pressures that exist to influence allele frequencies are not confined to a particular geographic area, and most likely the pathogen is exposed to lowly variable climatic conditions, hosts and cropping systems. On the other hand, a strong indication of gene flow was detected among East African populations. Although, this tends to reduce genetic differentiation among populations, if local selection and genetic drift within populations is sufficiently high it may permit emergence and spread of highly advantageous alleles. Thus, regional efforts to limit informal movement of planting materials may be necessary to prevent epidemics associated with exotic strains. The presence of two mating types in some locations indicates past signatures of evolution which may need further examination to understand. Being the first study on M. oryzae on rice in East Africa, the effect of the predicted climate change will need to be investigated further in subsequent studies. 


\section{Appendix 1}

Table S1: M. oryzae pathogenicity genes expressed in planta at 48 hours post inoculation

\begin{tabular}{|c|c|c|c|c|c|}
\hline Gene ID & Gene name & Phenotype & Description & $\begin{array}{l}\text { Nip35i } \\
\text { Log2FC }\end{array}$ & $\begin{array}{l}\text { Nip28i } \\
\text { Log2FC }\end{array}$ \\
\hline MGG_12337 & MAS3 & Reduced virulence & MAS3 protein & 15.03 & 12.27 \\
\hline MGG_04301 & PWL2 & $\begin{array}{l}\text { Effector (plant avirulence } \\
\text { determinant) }\end{array}$ & conserved hypothetical protein & 9.66 & 9.00 \\
\hline MGG_13863 & PWL1 & $\begin{array}{l}\text { Effector (plant avirulence } \\
\text { determinant) }\end{array}$ & conserved hypothetical protein & 9.58 & 9.34 \\
\hline MGG_04202 & GAS2/MAS3 & Reduced virulence & MAS3 protein & 9.50 & 10.63 \\
\hline MGG_10510 & MGG_10510 & Reduced virulence & ribonuclease $\mathrm{T} 2$ & 10.47 & 8.99 \\
\hline MGG_08735 & MoRgs5 & Unaffected pathogenicity & conserved hypothetical protein & 8.47 & 5.77 \\
\hline MGG_15370 & AVR-Pita & $\begin{array}{l}\text { Effector (plant avirulence } \\
\text { determinant) }\end{array}$ & Metalloproteinase & 8.07 & 7.83 \\
\hline MGG_12865 & MoHox7 & Loss of pathogenicity & conserved hypothetical protein & 6.91 & 6.09 \\
\hline MGG_13926 & MoRgs8 & Unaffected pathogenicity & conserved hypothetical protein & 6.81 & 8.18 \\
\hline MGG_04895 & ICL1 & Reduced virulence & isocitrate lyase & 6.58 & 5.75 \\
\hline MGG_10730 & $\begin{array}{l}\text { Calcium- } \\
\text { transporting } \\
\text { ATPase } 3\end{array}$ & Reduced virulence & calcium-transporting ATPase 3 & 5.96 & 5.26 \\
\hline MGG_05731 & $\begin{array}{l}\text { Peptidyl } \\
\text { prolyl } \\
\text { isomerase }\end{array}$ & Reduced virulence & $\begin{array}{l}\text { peptidyl-prolyl cis-trans } \\
\text { isomerase cyp } 15\end{array}$ & 4.71 & 4.72 \\
\hline MGG_09705 & NMR3 & Reduced virulence & $\begin{array}{l}\text { nucleoside-diphosphate-sugar } \\
\text { epimerase family protein }\end{array}$ & 4.60 & 6.23 \\
\hline MGG_05531 & MAC1 & Reduced virulence & hypothetical protein & 4.36 & 3.26 \\
\hline MGG_06148 & MFP1 & Reduced virulence & $\begin{array}{l}\text { peroxisomal hydratase- } \\
\text { dehydrogenase-epimerase }\end{array}$ & 4.05 & 3.13 \\
\hline MGG_04685 & MGG_04685 & Reduced virulence & conserved hypothetical protein & 3.45 & 2.82 \\
\hline MGG_01721 & CrAT1 & Loss of pathogenicity & carnitine $\mathrm{O}$-acetyltransferase & 3.35 & 3.02 \\
\hline MGG_04116 & MGG_04116 & Reduced virulence & $\begin{array}{l}\text { SH3 domain-containing } \\
\text { protein }\end{array}$ & 3.30 & 2.74 \\
\hline MGG_06939 & $\mathrm{ABC} 1$ protein & & $\mathrm{ABC} 1$ protein & 3.25 & 2.55 \\
\hline MGG_04628 & MoCYP51A & Reduced virulence & cytochrome P450 51 & 3.22 & 2.52 \\
\hline MGG_00527 & EMP1 & Reduced virulence & hypothetical protein & 3.21 & 2.81 \\
\hline MGG_01099 & $\begin{array}{l}\text { PTH2 } \\
\text { endo-1,4- } \\
\text { beta-xylanase } \\
\text { [GH10 }\end{array}$ & Loss of pathogenicity & peptidyl-tRNA hydrolase 2 & 3.17 & 3.11 \\
\hline MGG_05464 & family] & Reduced virulence & endo-1,4-beta-xylanase & 3.16 & 5.94 \\
\hline MGG_02376 & HDL1 & Unaffected pathogenicity & hormone-sensitive lipase & 3.07 & -3.92 \\
\hline MGG_12128 & Mid1 & Mixed outcome & $\begin{array}{l}\text { calcium influx-promoting } \\
\text { protein ehs } 1\end{array}$ & 2.98 & 2.05 \\
\hline MGG_08624 & ERG2 & $\begin{array}{l}\text { chemistry target - } \\
\text { phenotype unknown }\end{array}$ & C-8 sterol isomerase & 2.96 & -2.61 \\
\hline MGG_08628 & MGG_08628 & Reduced virulence & 3'-5' exoribonuclease CSL4 & 2.94 & 2.24 \\
\hline MGG_09299 & PAS1 & Unaffected pathogenicity & $\begin{array}{l}\text { peroxisome biosynthesis } \\
\text { protein (PAS1/Peroxin-1) }\end{array}$ & 2.78 & 2.56 \\
\hline MGG_02731 & MGG_02731 & Loss of pathogenicity & cell division control protein 42 & 2.77 & -3.06 \\
\hline MGG_07667 & Moatg17 & Loss of pathogenicity & autophagy-related protein 17 & 2.75 & 2.46 \\
\hline
\end{tabular}




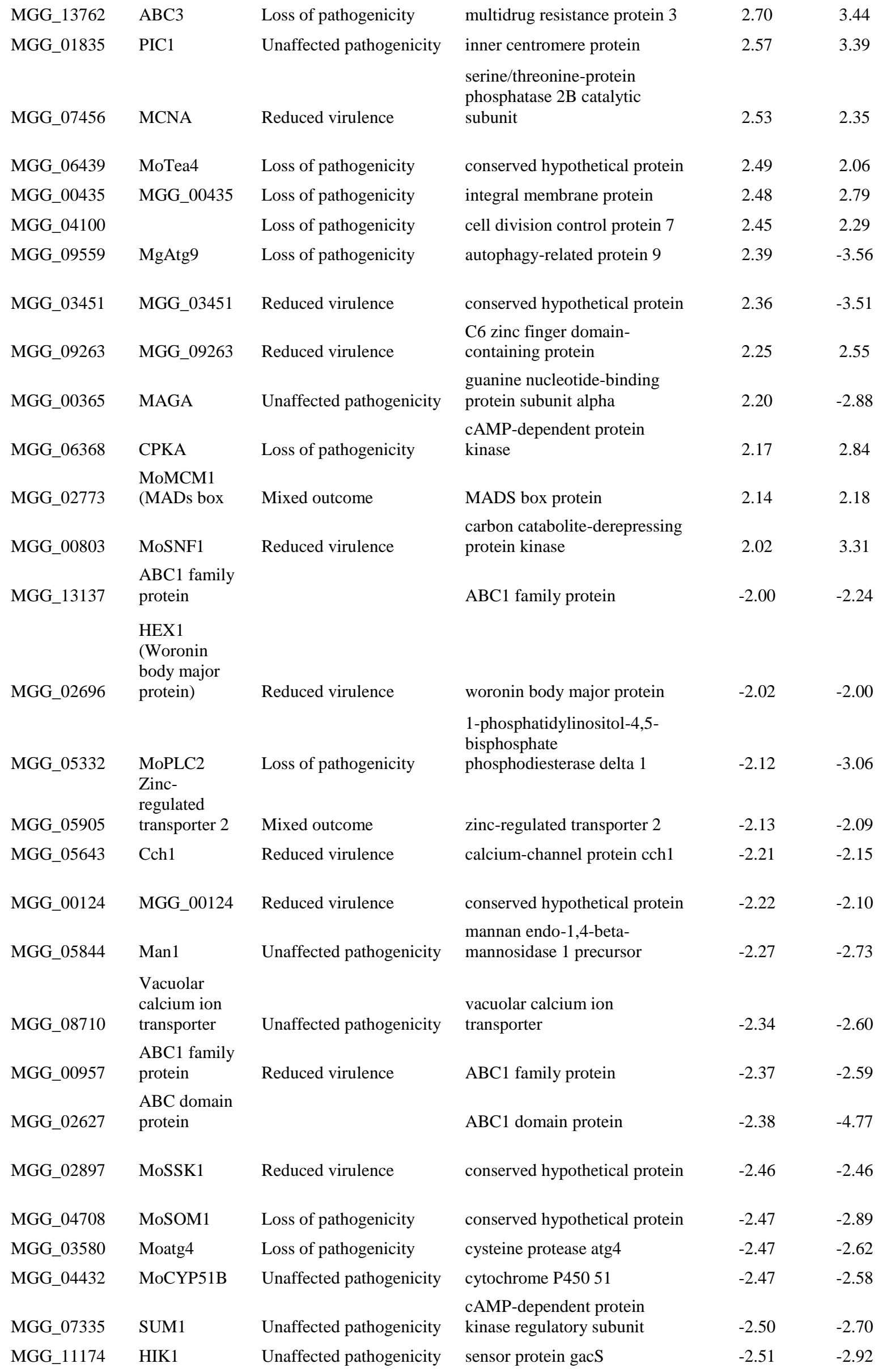




\begin{tabular}{|c|c|c|c|c|c|}
\hline MGG_09828 & Yvc1 & Mixed outcome & vacuolar conductance protein & -2.61 & -3.41 \\
\hline MGG_06951 & MGG_06951 & Reduced virulence & CAAX prenyl protease 1 & -2.63 & 2.30 \\
\hline MGG_12828 & Moatg15 & Loss of pathogenicity & conserved hypothetical protein & -2.64 & -3.25 \\
\hline MGG_04582 & MGG_04582 & Reduced virulence & conserved hypothetical protein & -2.67 & -2.73 \\
\hline MGG_02443 & MGG_02443 & Reduced virulence & conserved hypothetical protein & -2.69 & 2.18 \\
\hline MGG_00454 & Moatg13 & Reduced virulence & autophagy related protein $13 p$ & -2.78 & -3.88 \\
\hline MGG_05078 & $\begin{array}{l}\text { Calcium- } \\
\text { transporting } \\
\text { ATPase } 3\end{array}$ & Loss of pathogenicity & calcium-transporting ATPase 3 & -2.85 & -3.75 \\
\hline MGG_06933 & $\mathrm{CNB}$ & Mixed outcome & calcineurin subunit B & -2.85 & -2.83 \\
\hline MGG_10315 & MPG1 & Reduced virulence & Hydrophobin & -2.85 & -6.01 \\
\hline MGG_09471 & PTH9 & Reduced virulence & neutral trehalase & -2.88 & -3.29 \\
\hline MGG_14014 & Moplaa & Reduced virulence & $\begin{array}{l}\text { ubiquitin homeostasis protein } \\
\text { lub1 }\end{array}$ & -2.91 & -2.71 \\
\hline MGG_05133 & MoCRZ1 & Reduced virulence & $\begin{array}{l}\mathrm{C} 2 \mathrm{H} 2 \text { type zinc finger domain- } \\
\text { containing protein }\end{array}$ & -3.01 & -2.70 \\
\hline MGG_05174 & MGG_05174 & Reduced virulence & $\begin{array}{l}\text { pinin/SDK/memA domain- } \\
\text { containing protein }\end{array}$ & -3.04 & -3.00 \\
\hline MGG_01730 & MoHox3 & reduced virulence & conserved hypothetical protein & -3.05 & -2.97 \\
\hline MGG_10633 & MoAP1 & Loss of pathogenicity & AP-1 complex subunit mu-1 & -3.10 & -3.36 \\
\hline MGG_00466 & $\mathrm{Cdc} 42$ & Reduced virulence & cell division control protein 42 & -3.12 & -3.00 \\
\hline MGG_12005 & Spf1 & Mixed outcome & cation-transporting ATPase 4 & -3.13 & -2.96 \\
\hline MGG_13239 & 7,8-LDS & Unaffected pathogenicity & linoleate diol synthase & -3.14 & -2.62 \\
\hline MGG_02755 & NUT1 & Reduced virulence & $\begin{array}{l}\text { nitrogen regulatory protein } \\
\text { NUT1 }\end{array}$ & -3.14 & -3.67 \\
\hline MGG_02622 & TGL1-1 & Unaffected pathogenicity & sterol esterase TGL1 & -3.19 & -3.29 \\
\hline MGG_02370 & Mir1 & Unaffected pathogenicity & $\begin{array}{l}\text { mitochondrial phosphate } \\
\text { carrier protein }\end{array}$ & -3.19 & -2.85 \\
\hline MGG_01236 & SLP 1 & Reduced virulence & $\begin{array}{l}\text { WD repeat-containing protein } \\
\text { slp1 }\end{array}$ & -3.26 & -2.76 \\
\hline MGG_04204 & MAGC & Unaffected pathogenicity & $\begin{array}{l}\text { guanine nucleotide-binding } \\
\text { protein alpha-2 subunit }\end{array}$ & -3.28 & -3.91 \\
\hline MGG_03146 & MoRgs2 & Unaffected pathogenicity & conserved hypothetical protein & -3.37 & -2.88 \\
\hline MGG_03638 & Moatg24 & Unaffected pathogenicity & autophagy-related protein 24 & -3.41 & -4.48 \\
\hline MGG_02252 & BUF1 & Loss of pathogenicity & $\begin{array}{l}\text { tetrahydroxynaphthalene } \\
\text { reductase }\end{array}$ & -3.41 & -2.61 \\
\hline MGG_06033 & MoMSB2 & Loss of pathogenicity & hypothetical protein & -3.46 & -4.22 \\
\hline MGG_04621 & MGG_04621 & Reduced virulence & conserved hypothetical protein & -3.54 & -3.03 \\
\hline MGG_02436 & MGG_02436 & Reduced virulence & conserved hypothetical protein & -3.55 & -5.32 \\
\hline MGG_12175 & SSM1 & Reduced virulence & tyrocidine synthetase 1 & -3.55 & -3.71 \\
\hline MGG_06847 & Annexin A7 & Loss of pathogenicity & annexin $\mathrm{A} 7$ & -3.60 & -4.04 \\
\hline MGG_00447 & $\begin{array}{l}\mathrm{ABC} 2 \\
\text { (Brefeldin A } \\
\text { resistance } \\
\text { protein) }\end{array}$ & Unaffected pathogenicity & brefeldin A resistance protein & -3.61 & -2.75 \\
\hline MGG_08212 & Moatf1 & Reduced virulence & $\begin{array}{l}\text { BZIP transcription factor } \\
\text { (AtfA) }\end{array}$ & -3.62 & -3.70 \\
\hline
\end{tabular}




\begin{tabular}{|c|c|c|c|c|c|}
\hline MGG_09125 & MoSHO1 & Reduced virulence & osmosensor protein & -3.67 & -4.31 \\
\hline MGG_05201 & $\begin{array}{l}\text { MGB1 } \\
\text { (guanine } \\
\text { binding } \\
\text { nucleitide } \\
\text { protein) }\end{array}$ & Loss of pathogenicity & $\begin{array}{l}\text { guanine nucleotide-binding } \\
\text { protein subunit beta }\end{array}$ & -3.76 & -3.28 \\
\hline MGG_02799 & CHS5 & Loss of pathogenicity & conserved hypothetical protein & -3.76 & -2.89 \\
\hline MGG_04556 & MGG_04556 & Reduced virulence & alcohol dehydrogenase 1 & -3.82 & -3.72 \\
\hline MGG_01822 & $\begin{array}{l}\text { OSM1 } \\
\text { (MAPK- } \\
\text { HOG1) }\end{array}$ & Unaffected pathogenicity & $\begin{array}{l}\text { mitogen-activated protein } \\
\text { kinase HOG1 }\end{array}$ & -3.84 & -3.88 \\
\hline MGG_03726 & MoRgs3 & Reduced virulence & conserved hypothetical protein & -3.87 & -3.57 \\
\hline MGG_07289 & Gph1 & Mixed outcome & glycogen synthase & -3.93 & -4.10 \\
\hline MGG_04137 & MGG_04137 & Reduced virulence & $\begin{array}{l}\text { CTLH domain-containing } \\
\text { protein }\end{array}$ & -3.93 & -3.05 \\
\hline MGG_00750 & NOX1 & Loss of pathogenicity & $\begin{array}{l}\text { cytochrome b- } 245 \text { heavychain } \\
\text { subunit beta }\end{array}$ & -3.96 & -4.36 \\
\hline MGG_03530 & MGG_03530 & Reduced virulence & conserved hypothetical protein & -4.03 & -4.71 \\
\hline MGG_10017 & NMR1 & Reduced virulence & NmrA family protein & -4.05 & -2.65 \\
\hline MGG_05344 & MSP1 & Reduced virulence & conserved hypothetical protein & -4.06 & -6.27 \\
\hline MGG_03860 & $\begin{array}{l}\text { TPS1 } \\
\text { (alpha,alpha- } \\
\text { trehalose- } \\
\text { phosphate } \\
\text { synthase 1) }\end{array}$ & Loss of pathogenicity & $\begin{array}{l}\text { alpha,alpha-trehalose- } \\
\text { phosphate synthase } 1\end{array}$ & -4.13 & -3.93 \\
\hline MGG_09551 & CHS1 & Reduced virulence & chitin synthase 3 & -4.17 & -4.22 \\
\hline MGG_07528 & PTH3 & Loss of pathogenicity & $\begin{array}{l}\text { imidazoleglycerol-phosphate } \\
\text { dehydratase }\end{array}$ & -4.21 & -2.63 \\
\hline MGG_04428 & $\begin{array}{l}\text { ACE1 (zinc } \\
\text { finger } \\
\text { transcription } \\
\text { factor) }\end{array}$ & $\begin{array}{l}\text { Effector (plant avirulence } \\
\text { determinant) }\end{array}$ & $\begin{array}{l}\text { zinc finger transcription factor } \\
\text { ace } 1\end{array}$ & -4.26 & -3.97 \\
\hline MGG_07460 & MoHyr1 & Reduced virulence & peroxiredoxin HYR1 & -4.39 & -4.44 \\
\hline MGG_02423 & MGG_02423 & Loss of pathogenicity & $\begin{array}{l}\text { ER lumen protein retaining } \\
\text { receptor } 2\end{array}$ & -4.39 & -4.17 \\
\hline MGG_08600 & PIC5 & Reduced virulence & $\begin{array}{l}\text { mannose-P-dolichol utilization } \\
\text { defect } 1 \text { protein }\end{array}$ & -4.39 & -3.10 \\
\hline MGG_09312 & $\begin{array}{l}\text { Conserved } \\
\text { hypothetical } \\
\text { protein }\end{array}$ & Reduced virulence & conserved hypothetical protein & -4.41 & -4.58 \\
\hline MGG_09912 & MoCMK1 & Reduced virulence & $\begin{array}{l}\text { calcium/calmodulin-dependent } \\
\text { protein kinase }\end{array}$ & -4.47 & -4.47 \\
\hline MGG_09565 & PMK1 & Loss of pathogenicity & $\begin{array}{l}\text { mitogen-activated protein } \\
\text { kinase }\end{array}$ & -4.66 & -3.74 \\
\hline MGG_04629 & MGG_04629 & Reduced virulence & integral membrane protein & -4.75 & -4.71 \\
\hline MGG_04853 & MoHox1 & reduced virulence & Homeoprotein & -4.86 & -4.59 \\
\hline MGG_07259 & MGG_07259 & Reduced virulence & conserved hypothetical protein & -4.92 & -3.75 \\
\hline MGG_04489 & MNH6 & Reduced virulence & nucleosome binding protein & -4.95 & -3.93 \\
\hline MGG_05933 & TGL3-1 & Unaffected pathogenicity & lipase 4 & -5.21 & -2.14 \\
\hline MGG_03284 & MGG_03284 & Reduced virulence & DNA mismatch repair protein & -5.23 & -3.68 \\
\hline
\end{tabular}




\begin{tabular}{|c|c|c|c|c|c|}
\hline MGG_01707 & MGG_01707 & Reduced virulence & conserved hypothetical protein & -5.47 & -5.06 \\
\hline MGG_04212 & OMO1 & Loss of pathogenicity & L-ornithine 5-monooxygenase & -5.81 & -5.53 \\
\hline MGG_13013 & CHS6 & Loss of pathogenicity & chitin synthase 8 & -6.17 & -5.45 \\
\hline MGG_05132 & MoSKN7 & Unaffected pathogenicity & conserved hypothetical protein & -6.62 & -2.22 \\
\hline MGG_09098 & & & endo-1,4-beta-xylanase & 11.97 & 12.54 \\
\hline & $\begin{array}{l}\mathrm{ABC} 1 \\
\text { transporter }\end{array}$ & & & & \\
\hline MGG_13624 & CDR4 & & $\mathrm{ABC}$ transporter $\mathrm{CDR} 4$ & 4.15 & - \\
\hline MGG_04486 & Moatg11 & Unaffected pathogenicity & $\begin{array}{l}\text { Taz1-interacting factor } 1 \\
\text { (TAF1) }\end{array}$ & 3.46 & - \\
\hline MGG_11712 & MoHox6 & reduced virulence & conserved hypothetical protein & 3.21 & - \\
\hline MGG_07437 & MoHox 5 & reduced virulence & conserved hypothetical protein & 2.84 & - \\
\hline MGG_12958 & $\begin{array}{l}\text { MST12 } \\
\text { (Transcription } \\
\text { factor SteA) }\end{array}$ & Loss of pathogenicity & transcription factor steA & 2.75 & - \\
\hline MGG_00345 & MoRIM15 & Reduced virulence & $\begin{array}{l}\text { serine/threonine-protein kinase } \\
\text { RIM15 }\end{array}$ & 2.69 & - \\
\hline MGG_09898 & MGG_09898 & Adenylate cyclase & adenylate cyclase & 2.66 & - \\
\hline MGG_11454 & $\begin{array}{l}\text { Vacuolar } \\
\text { calcium ion } \\
\text { transporter }\end{array}$ & Unaffected pathogenicity & $\begin{array}{l}\text { vacuolar calcium ion } \\
\text { transporter }\end{array}$ & 2.55 & - \\
\hline MGG_03459 & PTH8 & Reduced virulence & autophagy-related protein 26 & 2.33 & - \\
\hline MGG_06064 & CON7 & Reduced virulence & chitin synthase D & 2.31 & - \\
\hline MGG_06971 & MoSFl1 & Reduced virulence & $\begin{array}{l}\text { flocculation suppression } \\
\text { protein }\end{array}$ & 2.28 & - \\
\hline MGG_00883 & MGG_00883 & Loss of pathogenicity & $\begin{array}{l}\text { mitogen activated protein } \\
\text { kinase kinase kinase } 3\end{array}$ & 2.18 & - \\
\hline MGG_01215 & COM1 & Reduced virulence & conserved hypothetical protein & 2.13 & - \\
\hline MGG_05664 & $\mathrm{PdeH}$ & Mixed outcome & $\begin{array}{l}\text { cAMP-specific 3',5'-cyclic } \\
\text { phosphodiesterase 7B }\end{array}$ & 2.12 & - \\
\hline MGG_05199 & MST50p & Loss of pathogenicity & protein kinase regulator Ste50 & 2.06 & - \\
\hline MGG_01818 & MAGB & Reduced virulence & $\begin{array}{l}\text { guanine nucleotide-binding } \\
\text { protein alpha-3 subunit }\end{array}$ & 2.05 & - \\
\hline MGG_01196 & Kin4 & Mixed outcome & $\begin{array}{l}\text { serine/threonine-protein kinase } \\
\text { MARK2 }\end{array}$ & 2.03 & - \\
\hline MGG_07015 & MGG_07015 & Reduced virulence & DNA repair protein $\operatorname{Rad} 7$ & -2.09 & - \\
\hline MGG_04066 & Neol & Loss of pathogenicity & $\begin{array}{l}\text { phospholipid-transporting } \\
\text { ATPase } 1\end{array}$ & -2.28 & - \\
\hline MGG_07297 & Moatg7 & Loss of pathogenicity & autophagy-related protein 7 & -2.40 & - \\
\hline MGG_04547 & & & endoglucanase II & - & 11.18 \\
\hline MGG_01542 & XYL2 & Unaffected pathogenicity & endo-1,4-beta-xylanase & - & 9.00 \\
\hline MGG_09618 & $\begin{array}{l}\text { MoRgs6 } \\
\text { endo-1,4- } \\
\text { beta-xylanase } \\
\text { precursor } \\
\text { [GH10 }\end{array}$ & Unaffected pathogenicity & conserved hypothetical protein & - & 7.38 \\
\hline MGG_14243 & family] & Reduced virulence & precursor & - & 6.11 \\
\hline MGG 07868 & $\begin{array}{l}\text { endo-1,4- } \\
\text { beta-xylanase } \\
\end{array}$ & Reduced virulence & endo-1 4-beta-xylanase & - & 306 \\
\hline
\end{tabular}


family]

\begin{tabular}{|c|c|c|c|c|c|}
\hline MGG_05871 & PTH11 & Reduced virulence & integral membrane protein & - & 2.86 \\
\hline MGG_01204 & MIG1 & Loss of pathogenicity & $\begin{array}{l}\text { MADS-box MEF2 type } \\
\text { transcription factor }\end{array}$ & - & 2.29 \\
\hline MGG_11899 & MGG_11899 & Reduced virulence & $\begin{array}{l}\text { SH3 domain-containing } \\
\text { protein }\end{array}$ & - & 2.06 \\
\hline MGG_01748 & MGG_01748 & Reduced virulence & conserved hypothetical protein & - & 2.06 \\
\hline MGG_01381 & $\begin{array}{l}\text { Calcium } \\
\text { permease }\end{array}$ & Mixed outcome & calcium permease & - & -2.03 \\
\hline MGG_00184 & HTF1 & Unaffected pathogenicity & conserved hypothetical protein & - & -2.04 \\
\hline MGG_06421 & Tos 3 & Unaffected pathogenicity & $\begin{array}{l}\text { calcium/calmodulin-dependent } \\
\text { protein kinase kinase } 2\end{array}$ & - & -2.11 \\
\hline MGG_07971 & Pmc1 & Mixed outcome & calcium-transporting ATPase 1 & - & -2.13 \\
\hline MGG_06559 & NOX2 & Loss of pathogenicity & $\begin{array}{l}\text { cytochrome b- } 245 \text { heavychain } \\
\text { subunit beta }\end{array}$ & - & -3.50 \\
\hline
\end{tabular}


Appendix 2

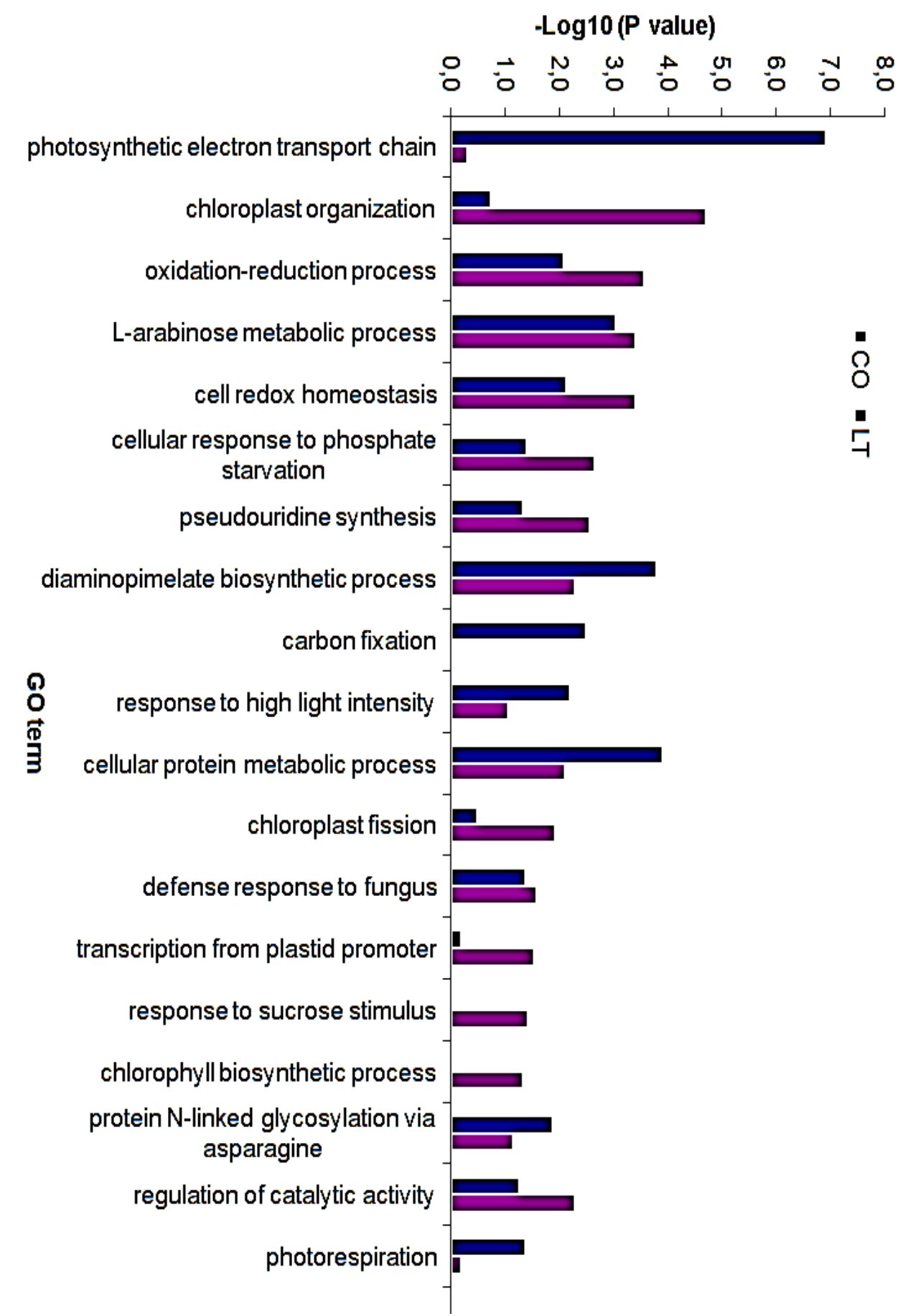

Figure S1: Significant Gene Ontology (GO) enriched terms from differentially up regulated genes (fold change 4; FDR, 0.05) in plants exposed to $35^{\circ} \mathrm{C}$ for 7 days. Comparison of the two genetic backgrounds; Blue bars, $\mathrm{CO}+35^{\circ} \mathrm{C}$; purple bars, $\mathrm{LT}+35^{\circ} \mathrm{C}$. 


\section{Acknowledgements}

I am indebted to many individuals who have enabled the completion of this dissertation. My deepest gratitude goes to my supervisors, Prof. Dr. Andreas von Tiedemann and Prof. Dr. Kerstin Wydra for the scientific mentorship they provided to me. Prof. von Tiedemann is a caring and patient individual whose contribution to my scientific career has been very evident. I feel privileged to have worked with him for two main reasons: first, his kind nature and willingness to assist students and second, his exceptional knowledge of plant pathology and scientific writing expertise. His interest in helping students, approachableness and outgoing nature makes association with him enjoyable. I am grateful to Prof. Wydra. Without her favor, I would not have got this PhD opportunity. She was always motivational, willing to answer my questions and provide advice about my research whenever I requested; her contribution to the success of this research is highly valued. I would like to thank Dr. Christian Möllers for accepting to be in my dissertation evaluation committee. I am grateful to Dr. Birger Koopmann. His knowledge in molecular plant pathology provided me with a clear understanding of many aspects in my research. My research would not have been possible without the assistance of technicians. My sincere thanks go to Evelin Vorbeck, Dagmar Tacke, Kerstin Höch, Marian Süß, and Daniel Kretzschmar for their technical support. I am grateful to my fellow graduate students. We worked together and supported each other in areas of need, and that was a great privilege. I am grateful to my friend, Merle Tränkner, from the Institute of Applied Plant Nutrition; she significantly contributed to my research and participated in fine tuning my dissertation. I would like to thank Prof. Dr. Ulrich Schaffrath, Dr. Hassan Ghareeb, Dr. Mark Winter and Dr. Anke Sirrenberg for their valuable comments that helped improve the quality of this dissertation. My sincere thanks also go to the Microarray and Deep-Sequencing Core Facility of the Developmental Biochemistry, University Medical Center Göttingen (UMG) for performing illumina sequencing and participation in RNA-seq data analysis. Finally, I owe deepest gratitude to my family, especially, my wife Sarah Onaga. She tolerated my absence from home for the last 4 years and prayed for my success. Above all, I would like to express my profound gratitude to the Almighty God for his love, mercy, guidance and safeguard. He has brought me this far.

This research was supported by BMZ funded project "Mitigating the impact of climate change on rice diseases in East Africa" which was spearheaded by AfricaRice center. The contribution of these institutions to my education and research is greatly appreciated. 


\section{Publications}

Onaga, G., Wydra, K., Koopmann, B., Sere, Y., Ghareeb, H., Von Tiedemann, A. (2016) High temperature effects on Pi54 conferred resistance to Magnaporthe oryzae in two genetic backgrounds of Oryza sativa (manuscript submitted)

Onaga, G., Wydra, K., Koopmann, B., Sere, Y., Von Tiedemann, A. (2016) Elevated temperature increases inplanta expression levels of virulence related genes in Magnaporthe oryzae and compromises resistance in Nipponbare (manuscript accepted with revisions)

Onaga, G., Wydra, K., Koopmann, B., Sere, Y., Von Tiedemann, A. (2014) Population Structure, Pathogenicity, and Mating Type Distribution of Magnaporthe oryzae Isolates from East Africa. Phytopathology 105(8):1137-45.

\section{Selected conference contributions}

Onaga, G., Wydra, K., Koopmann, B., Sere, Y., Von Tiedemann, A. (2013) Genomic studies on resistance of rice to blast, Magnaporthe oryzae, at rising temperature. Presented at the $10^{\text {th }}$ International Congress of Plant Pathology, Beijing, China. See in the book of Abstracts. pg.333.

Onaga, G., Wydra, K., Koopmann, B., Sere, Y., Von Tiedemann, A. (2013) Population structure of Magnaporthe oryzae, the causal agent of rice blast in East Africa. $3^{\text {rd }}$ Africa Rice Congress, Yaoundé, Cameroon. See the book of Abstracts. Pg.190.

Onaga, G., Wydra, K., Koopmann, B., Sere, Y., Von Tiedemann, A. (2012) Effects of High Temperature on R Gene Mediated Resistance to Rice Blast in two Genetic Backgrounds of Rice. TROPENTAG 2012. 
Curriculum vitae

\section{Personal details}

Full Name Geoffrey Onaga

Date of birth 23.02.1978

Place of Birth Ngora, Uganda

Secondary education

1997/98 Teso College Aloet, Soroti

1993/96 Rock High School, Tororo

1985/92 St. Kizito's Primary School, Tororo

\section{Tertiary Education}

2014 PhD studies, Georg-August-University Göttingen, Germany.

2010 MSc. Crop Science, Makerere University, Kampala, Uganda.

2004 Bachelor of Science in Agriculture (Hons), Makerere University, Kampala, Uganda. 


\section{Declaration}

I hereby, declare that this dissertation was undertaken independently and without any unaccredited aid.

Göttingen, 28.05.2014 